\title{
From inside-out to outside-in: cortical lamination development in the Reelin-deficient neocortex
}

\author{
Dissertation \\ for the award of the degree \\ "Doctor rerum naturalium" \\ Faculty of Biology \\ of the Georg-August-Universität Göttingen
}

within the doctoral program Molecular Physiology of the Brain

of the Georg-August University School of Science (GAUSS)

submitted by

Nieves Mingo Moreno

Born in Madrid, Spain

Göttingen, 2018 


\section{Thesis Committee}

\section{Prof. Dr. Jochen F. Staiger}

Institute for Neuroanatomy, University Medical Center, Göttingen

Prof. Dr. Thomas Dresbach

Department of Anatomy and Embryology, University Medical Center, Göttingen

Prof. Dr. Ahmed Mansouri

Department of Molecular Cell Biology, MPI for Biophysical Chemistry, Göttingen

Department of Clinical Neurophysiology, Georg-August-Universität Göttingen

\section{Members of the Examination Board}

First Referee:

Prof. Dr. Jochen F. Staiger

Institute for Neuroanatomy, University Medical Center, Göttingen

Second Referee:

Prof. Dr. Thomas Dresbach

Department of Anatomy and Embryology, University Medical Center, Göttingen

\section{Further members of the Examination Board}

Prof. Dr. Ahmed Mansouri

Department of Molecular Cell Biology, MPI for Biophysical Chemistry, Göttingen Department of Clinical Neurophysiology, Georg-August-Universität Göttingen

Dr. Camin Dean

Trans-synaptic Signaling group, European Neuroscience Institute, Göttingen

Prof. Dr. Gregor Eichele

Department of Genes and Behaviour, MPI for Biophysical Chemistry, Göttingen

Prof. Dr. Anastassia Stoykova

Department of Genes and Behaviour, MPI for Biophysical Chemistry, Göttingen

Date of the oral examination: 23 March 2018 
I, Nieves Mingo Moreno, hereby declare that this doctoral thesis has been written independently and with no other sources and aids than those quoted. All results presented here were the outcome of my own labour unless stated otherwise.

Nieves Mingo Moreno

February $8^{\text {th }}, 2018$

Göttingen, Germany 


\section{Table of Contents}

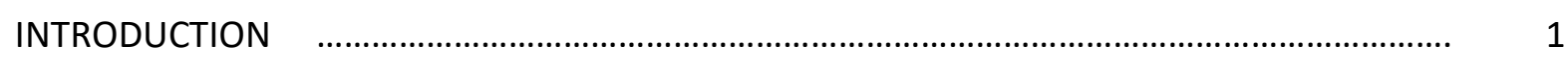

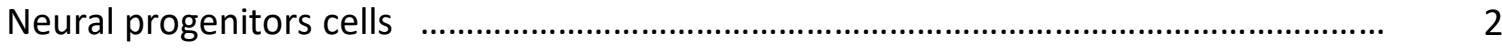

Inside-out cortical lamination $\quad$ …………………………..................................................

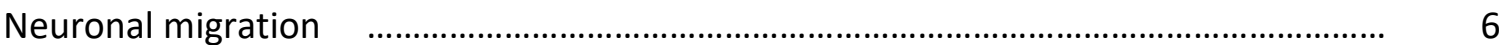

The reeler mouse: a mutant that questions the importance of cortical lamination $\quad \ldots \quad 8$

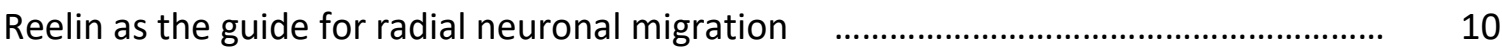

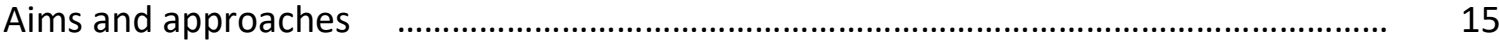

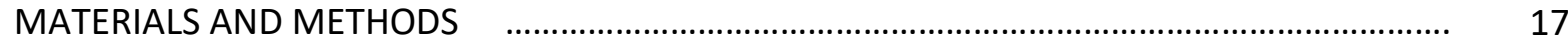

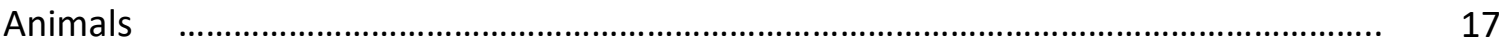

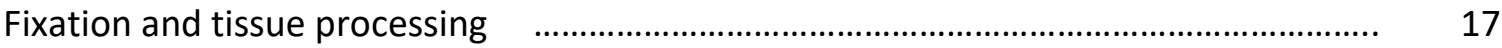

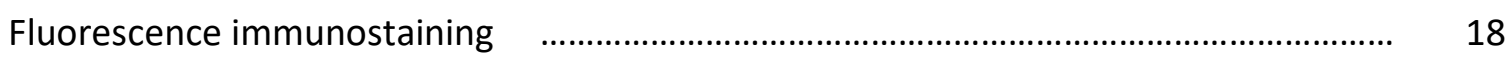

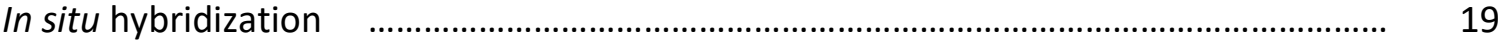

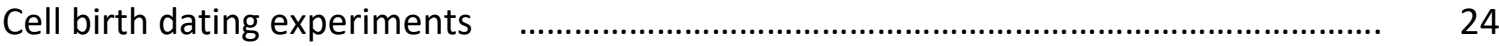

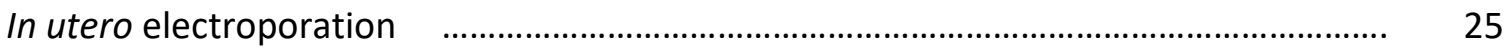

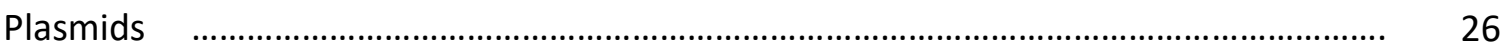

Transfection, immunoblotting and immunocytochemistry $\quad$............................................ 27

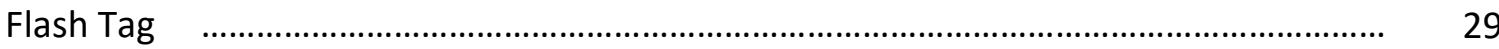

Image acquisition $\quad$....................................................................................................... 29

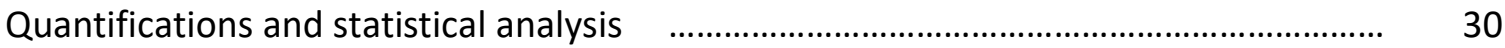

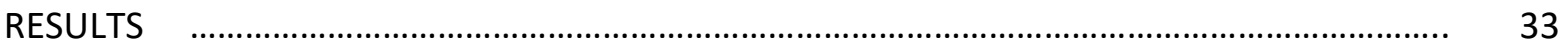

The many faces of the reeler cortex

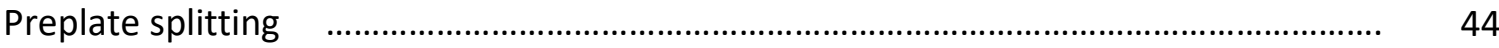

Neocortical histogenesis of the Reelin-deficient brain during development $\quad$................ $\quad 49$

Cell birth dating labeling principle $\quad$................................................................................ $\quad 50$

Progression of cortical (de)lamination in the S1BF $\quad$...................................................... 54

Behavior of early-born neurons $\quad$..................................................................... 56

Infragranular vs. supragranular cell distribution $\quad$......................................................... 56

Tracing of time-locked cohorts of newborn neurons 
Defining the first progeny of FT cells

Tracing of early-born neurons $\quad$................................................................................... 65

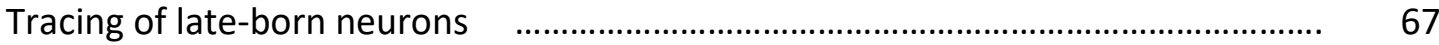

Neuronal positioning and cell fate of layer II/III cells postnatally $\quad$............................. $\quad 69$

How do we get here? Morphology of late-born migrating neurons $\quad$................................. 76

Early stages of migration ………………………........................................................... 76

Late stages of migration .................................................................................................. 77

Glial scaffold and Reelin ........................................................................................... 81

Glial fibers

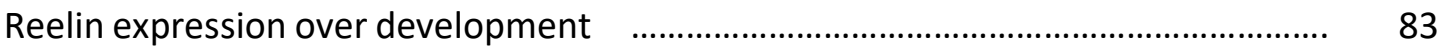

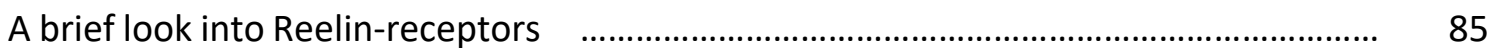

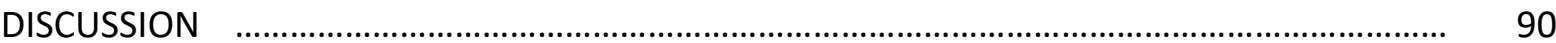

Adult cortical lamination in the absence of Reelin $\quad$....................................................... 90

Preplate splitting $\quad$...................................................................................................... 92

Neocortex assembly: cell birth dating analysis $\quad$............................................................. 95

Neuronal migration of late-born neurons in the Reelin deficient neocortex

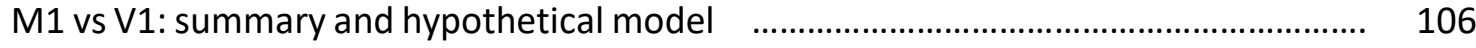

Reelin and the reeler cortex - New findings, yet more questions $\quad \ldots \ldots \ldots \ldots \ldots \ldots \ldots . . . . . . . . . . . . . . \quad 109$

SUMMARY 


\section{Figures}

Figure 1. Development of cortical lamination $\quad$...................................................................... 5

Figure 2. Neuronal migration during brain development in rodents $\quad$.................................. 7

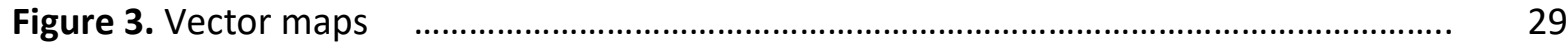

Figure 4. Disturbed cortical layering of the Reelin-deficient neocortex in an areaspecific fashion

Figure 5. Marker-based phenotyping in the frontal association area and the primary motor cortex

Figure 6. Marker-based phenotyping in sensory areas: primary somatosensory cortex, primary auditory cortex and primary visual cortex

Figure 7. Differential (mis)lamination in cortical areas in the adult reeler neocortex

Figure 8. Radial distribution of layer-fate marker expressing neurons in a given cortical column in reeler

Figure 9. Cell density and cortical thickness in wild type and Reelin-deficient mice

Figure 10. Cortical (mis)laminated patterns range from a quasi- inside-out in rostral sections, massive intermingling intermediately, to a quasi- inversion caudally

Figure 11. Identification of molecular markers specific for Cajal-Retzius and subplate cells

Figure 12. Preplate splitting is altered in rostral areas but does not occur in caudal regions

Figure 13. Cell birth dating labeling principle (I)

Figure 14. Cell birth dating labeling principle (II)

Figure 15. Development of cortical (de)lamination in the S1BF of reeler mice

Figure 16. Distribution of E13.5-born neurons during development in the M1, S1 and

V1 of wild type and reeler cortices

Figure 17. Development of opposing laminated patters in M1 and V1 in reeler 60

Figure 18. The progenitor pool is preserved in reeler

Figure 19. Labeling of apical-VZ- and SVZ/ basal-VZ - derived progeny by Flash Tag and BrdU 64

Figure 20. Tracing of early-born FT-labeled neurons 66

Figure 21. Tracing of late-born FT-labeled neurons 68 
Figure 22. Cell distribution of layer II/III VZ- and VZ/SVZ- derived in the premature cortex in P0 wild type and reeler mice

Figure 23. Final cortical location of layer II/III cells in wild type and reeler 72

Figure 24. Cell fate of layer II/III cells in newborn wild type and reeler mice 74

Figure 25. Cell fate of layer II/III cells in P7 wild type and reeler mice 75

Figure 26. Morphology of late-born neurons appears normal at early stages of migration

Figure 27. Morphology of late-born neurons is affected while sailing through the cortex in the Reelin-deficient neocortex 80

Figure 28. Radial glial fibers in E15.5 and E17.5 wild type and reeler mice 82

Figure 29. Reelin expression in anterior and posterior sections of wild type brains 84

Figure 30. Validation of VLDLR and ApoER2 protein expression in pcDNA and pCIG2 constructs 86

Figure 31. Overexpression of Reelin receptors in migrating neurons in the VZ-IZ 88

Figure 32. Overexpression of Reelin receptors in migrating neurons in the IZ-CP 89

Figure 33. Area-dependent disruption of the neocortical lamination in Reelin-deficient mice

Figure 34. Radial migration of late-born neurons 108

\section{Tables}

Table 1. Genes selected for in situ hybridizations

Table 2. Primer sequence and probe length for in situ hybridation riboprobes 


\section{List of Abbreviations}

\begin{tabular}{|c|c|}
\hline ApoER2 & Low-density lipoprotein receptor-related protein 8 \\
\hline Au1 & Primary auditory cortex \\
\hline BrdU & 5-bromo-2'-deoxyuridine \\
\hline & Cytomegalovirus immediate early enhancer and chicken â actin promoter \\
\hline CA & fusion \\
\hline CldU & 5-chloro-2'-deoxyuridine \\
\hline $\mathrm{CP}$ & Cortical plate \\
\hline CSFE & Carboxyfluorescein succinimidyl ester \\
\hline EdU & 5-ethynyl-2'-deoxyuridine \\
\hline FrA & Frontal association area \\
\hline Hek293 & Human embryonic kidney cells 293 \\
\hline IdU & 5 -iodo-2'-deoxyuridine \\
\hline IP & Intermediate progenitor \\
\hline M1 & Primary motor cortex \\
\hline MAZ & Multipolar accumulation zone \\
\hline Neuro2A & Mouse neuroblastoma cell line \\
\hline PB & Phosphate buffer \\
\hline PBS & Phosphate-buffered saline \\
\hline PFA & Paraformaldehyde \\
\hline RGC & Radial glial cell \\
\hline S.O.C & Super optimal broth with catabolite repression \\
\hline S1BF & Primary somatosensory 'barrel' cortex \\
\hline
\end{tabular}


SSC

SVZ

TSA

V1

VLDLR

VZ

Au1
Standard saline citrate

Subventricular zone

Tyramide signal amplification

Primary visual cortex

Very low density lipoprotein receptor

Ventricular zone

Primary auditory cortex 


\section{INTRODUCTION}

The brain is an organ that has fascinated scientist for centuries, due to its beauty and its complexity. From a developmental perspective, the formation of this multifaceted and highly organized structure is nothing but a biological wonder.

The neocortex (also termed as neopallium or isocortex) is the region of the mammalian brain responsible for higher brain functions, such as cognition, memory, language, consciousness and sensory perception. It is divided into specialized areas, which are well-defined by a distinct cytoarchitecture and specific wiring patterns. For instance, sensory information is processed in the so-called sensory areas (which include the somatosensory cortex, visual cortex and auditory cortex), the execution of voluntary movement is controlled by the primary motor cortex, and stimulus integration is directed by the frontal association cortex. These areas lie along the anterior-posterior axis of the neocortex. Thus, the primary motor cortex reside in the frontal lobe, while the primary visual cortex is found in the occipital lobe, in the anterior and posterior poles of the brain respectively (Kandel et al., 2013).

The adult mammalian neocortex is shaped in a six-layered (or laminae) configuration, numbered from the brain surface (pia mater) to the white matter. These layers are defined by a discrete populations of projection neurons with unique gene expression profiles, morphology and neuronal connectivity, which lead to diverse functional properties (Ramon y Cajal, 1991) (reviewed in (Molyneaux et al., 2007)). Layer (L) I or the molecular layer hosts few scattered GABAergic interneurons and consists mostly of apical dendrites from pyramidal cells in deeper layers and horizontally oriented axons. Layer II/III (LII/III) is composed of small pyramidal cells that mediate intracortical communication. This layer can be divided into the external granular cell layer (LII) and the external pyramidal layer (LIII), and constitutes, with layer I, the supragranular layers. Layer IV (LIV), or the internal granular layer, comprises a large number of stellate and pyramidal neurons and is considered the main receiver of the thalamic input. In contrast, layer $\mathrm{V}$ (LV), also called the internal pyramidal layer, is composed of large pyramidal neurons and its projections constitute the most prominent output pathways of the cortex. And last, in the deepest aspects of the cortex, layer VI (LVI, or the polymorphic layer), holds a heterogeneous neuronal population and hosts axons from different cortical areas (Kandel et al., 2013). 
The assembling of the neocortex is choreographed through an appropriate sequence of neuronal production, migration and positioning during embryonic and early postnatal stages. There are two main classes of neurons in the cortex, projection neurons (excitatory neurons) and interneurons (inhibitory neurons), which differ in e.g. their origin, migratory routes, morphology and roles in the neuronal network. Inhibitory or GABAergic neurons are morphologically highly diverse, migrate tangentially and originate from the ventricular zone in the ganglionic eminence. Projection or glutamatergic neurons, have a typical pyramidal morphology, migrate radially and derivate from the progenitor pool in the ventricular zone of the dorsal telencephalon (Gupta et al., 2002; Marin et al., 2010). As projection neurons constitute the core of this particular research project, only this neuronal class will be further reviewed.

\section{NEURAL PROGENITOR CELLS}

Projection neurons are born from neural progenitor cells in the pseudostratified epithelium that lines the ventricles in the dorsal telencephalon (Rakic, 1982). In early developmental stages, progenitor cells divide symmetrically to amplify their pool (Takahashi et al., 1995). By the beginning of the neurogenesis, neuroepithelial cells transition into radial glial cells (RGCs). RGCs express Pax6 (pair-box gene 6) and astrocyte markers, such as the astrocyte-specific glutamate transporter (GLAST). These cells have a long ascending process, which branches upon their termination at the pial surface, and a short apical process, which contacts the ventricular wall and remains attached during mitosis. The nuclei of neuroepithelial and RGCs undergo apical-basal oscillations in the ventricular zone (VZ), known as the interkinetic nuclear migration (IKNM), in a cell-cycle dependent manner: cells in mitosis (M) are located apically while those in $\mathrm{G} 1$ reside at the basal surface (Sauer and Walker, 1959). RGCs start to divide asymmetrically, producing neurons and at the same time self-renewing the progenitor pool, either by the generation of another RGC or an intermediate progenitor (IP) (Gotz and Huttner, 2005; Miyata et al., 2004; Noctor et al., 2001) (Fig.1). IPs, or basal progenitors, lack obvious processes and are Tbr2-positive (T-Box Brain Protein 2). These neuronal precursor cells are located in the basal VZ or in the subventricular zone (SVZ) and give rise to two neuronal descendants by symmetric division. At the end of neurogenesis, progenitors will preferentially produce two postmitotic cells that differentiate into neurons, reducing the precursor pool. 


\section{INSIDE-OUT CORTICAL LAMINATION}

Murine neuronal layer formation occurs between the second week of the embryonic development and the first postnatal week. At embryonic day $(E)$ 11, pioneer neurons migrate from their sites of origin towards the outer brain surface and constitute a transient layer structure named the preplate (PP) (Sheppard and Pearlman, 1997). At E13.5, the earliest wave of excitatory neurons exits the VZ and migrates by somal translocation to the pial surface (Morest, 1970; Nadarajah et al., 2001a), splitting the previously formed preplate into the superficial marginal zone (MZ) and the deeper subplate (SP) (Marin-Padilla, 1978; Rakic, 1972a). Subsequent cohorts of projection neurons migrate, first by locomotion along the glial scaffold (Marin-Padilla, 1978; Miyata et al., 2001) and subsequently by somal translocation, bypassing earlier layers within the developing cortical plate. As layers are generated sequentially over development, the neocortex is shaped in an inside-out manner (Angevine and Sidman, 1961), where early-born neurons (e.g. LV cells) occupy deep layers and late-born neurons (e.g. LII/III) reside superficially (Angevine and Sidman, 1961; Rakic, 1974) (Fig.1).

\section{Pioneer neurons in the cortex: Cajal-Retzius and Suplate cells}

Cajal Retzius (CR) cells, termed after Santiago Ramón y Cajal and Gustav Retzius (Cajal, 1861; Retzius, 1893), correspond to a transient early-born glutamatergic neuronal population, which constitutes the principal source of the extracellular matrix glycoprotein Reelin during development (D'Arcangelo et al., 1995). CR cells are confined to the uppermost layer of the cerebral cortex, referred as the marginal zone during development (Marin-Padilla, 1978) and molecular layer or layer I in the adult neocortex. These cells are born between E10 and E12 in mice (Hevner et al., 2003a) and have multiple origin sites (review by (Meyer, 2010)), with the cortical hem being the main source of CR cells for the neocortex (Meyer et al., 2002; Yoshida et al., 2006). CR cells have a prominent role in cortical lamination (Frotscher, 1997a) and show clear signs of degeneration after the first postnatal week in mice (del Rio et al., 1995; Derer and Derer, 1990; Tissir et al., 2009). Although it has been assumed that CR cells disappear by apoptosis, a small fraction survives during adulthood (Chowdhury et al., 2010)

Subplate cells. Subplate (SP) cells constitute a group of pioneer neurons that shape a mostly transient layer between the cortical plate and the intermediate zone (Angevine and Sidman, 1961; Bystron et al., 2008). SP cells are generated from E11.5 to E12.5 in mice and have also heterogeneous origins (Hoerder-Suabedissen and Molnar, 2015; Pedraza et al., 2014), for 
instance they may originate from the medial ganglionic eminence. SP neurons have crucial roles in development and brain maturation. At embryonic stages they are involved in thalamocortical pathfinding (McConnell et al., 1989), while they are indispensable for the maturation of the LIV cells circuitry postnatally (Kanold and Shatz, 2006). A large proportion of SP cells disappear by apoptosis after perinatal development (Ferrer et al., 1990; Kostovic and Rakic, 1980), and, although still under discussion, the reminiscent SP cells are believed to constitute the LVI b (Bayer and Altman, 1990; Marx et al., 2017).

\section{Birth date and layer specification}

Given the cell heterogeneity of the developing brain, it is still under debate how neuronal fate is determined. The laminar fate of a certain projection neuron type is at least partially specified at the progenitor level, as neurons that share birth date reside in the same cortical layer. The dominance of the intrinsic-clock that postmitotic neurons might have by their time of origin is especially striking in transplantation experiments, as neurons migrate to the layer destined by their birthdate, even when placed in an environment that already determines other laminae (McConnell and Kaznowski, 1991). However, those experiments also showed that neuronal progenitors are gradually restricted to respond to layer-fate determinant cues, since only early progenitors, but not late progenitors, can give rise to a differently fated progeny when the local microenvironment commands so (Desai and McConnell, 2000; Shen et al., 2006). Fate-restriction was also supported by the finding that VZ- and SVZ- progenitors change their gene-expression profiles through development and their progeny, determined to a certain layer, maintains the expression of those transcription factors (Chen et al., 2005; Nieto et al., 2004). However, a recent study based on a mosaic analysis system (MADAM) retains the debate open, as it shows that clones from a particular progenitor occupy infragranular and supragranular layers (Gao et al., 2014).

Layer-specific markers. It was just recently that a large number of projects have expanded the number of markers whose expression is restricted to a certain layer or a neuronal subtype, which can be used, therefore, for their identification (Gong et al., 2003; Liu et al., 2000; Sugino et al., 2006; Visel et al., 2004). Representative examples of laminar- or neuronal subtype- specific genes are: Cux1 (layer II/III cells), Rorb (marker for LIV cells), Bcl11d or Ctip2 (high lexpression in LV), Er81 (LIV projection neurons) and Tbr1 (neurons located in LIV) (reviewed by (Molyneaux et al., 2007; Popovitchenko and Rasin, 2017). 


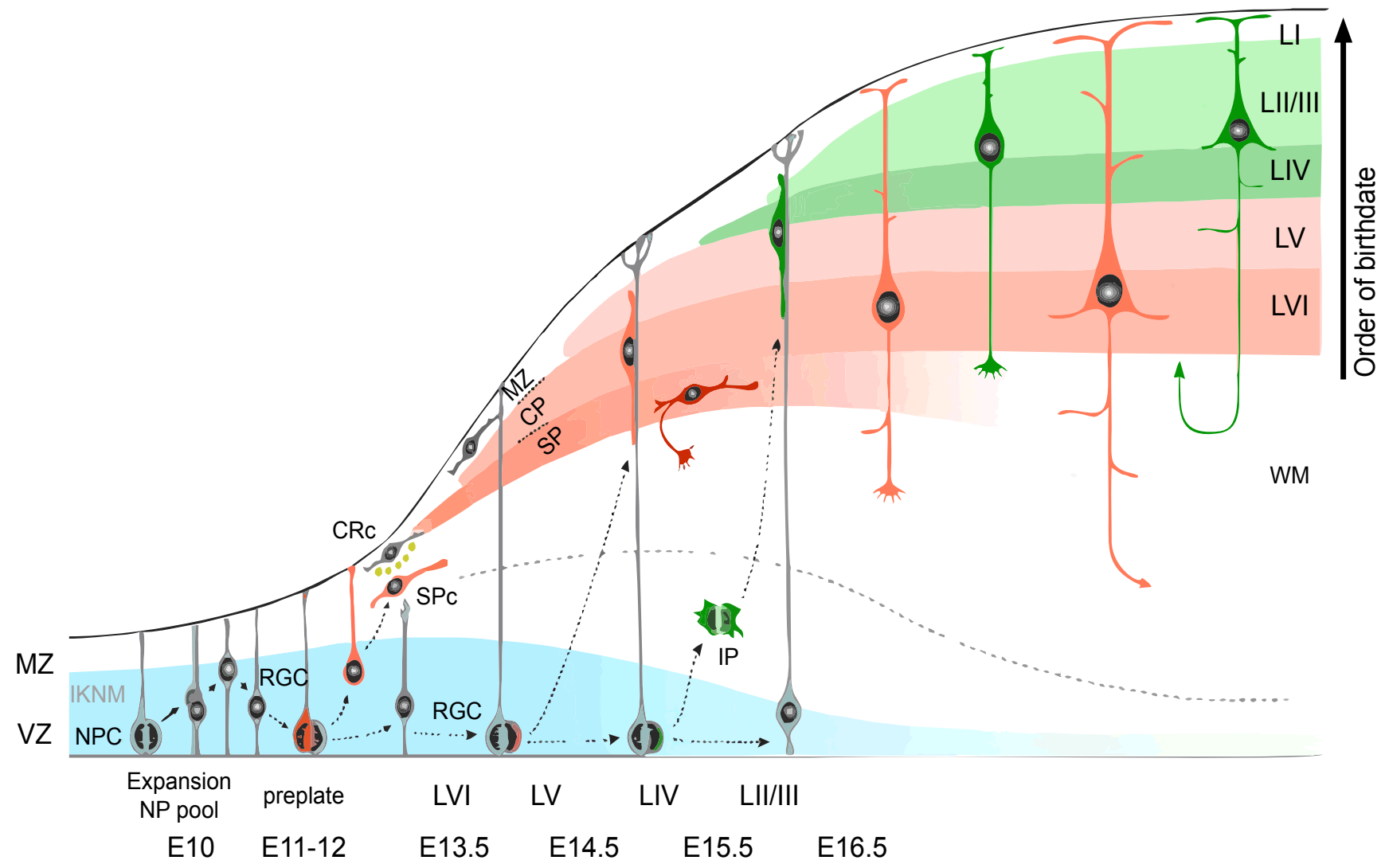

Neurogenesis

Figure 1. Development of cortical lamination. Neural progenitor cells (NPC) in the ventricular zone (VZ) divide first symmetrically to increase the progenitor pool. With the start of neurogenesis, NPC transition into radial glial cells (RGCs). RGCs divide asymmetrically to produce neurons and self-renew the progenitor population. At around E11, pioneer neurons migrate out from their sites of origin and constitute the preplate (PP). PP will be later split, by cohorts of projection neurons, in the upper marginal zone (MZ), populated by Cajal Retzius cells (CRc - main source of Reelin, shown in yellow), and the lower subplate (SP), inhabited by subplate cells (SPC). As neurogenesis progresses (E13.5-E16.5), cortical projection neurons are born in the VZ and migrate towards the pial surface in a sequential fashion, modeling the neocortex in an inside-out manner. Thus, neurons fated to deep layers (LVI and LV) are generated and travel first, followed by neurons destined to upper layers (LIV and LII/III). At the end of neurogenesis, RGCs switch to gliogenesis (not illustrated). Migration and positioning of interneurons is not shown. IKNM: interkinetic nuclear migration, CP: cortical plate, IP: intermediate progenitor, WM: white matter. Modified from (Kwan et al., 2012). 


\section{NEURONAL MIGRATION}

Pyramidal neurons encounter a remarkably long journey, from the proliferative zone to their particular location in the cerebral cortex, before they differentiate. Neuronal migration routes are complex and highly dynamic, as imply permanent cytoskeleton rearrangements and modifications in cell-cell interactions. Moreover, since proper lamination relays on a correct cell positioning, the process of neuronal migration is precisely regulated.

\section{Radial migration}

New-born projection neurons migrate in direction to the pia mater by two main styles of migration: somal translocation and glial-guided locomotion. Somal translocation is the leading mode of migration at early stages of development (Miyata et al., 2001; Morest, 1970; Nadarajah et al., 2001a), when the neocortex is just a thin wall and neurons have to cover only a short distance. Neurons using this way of migration (e.g. SP cells and LVI cells) typically have a long leading process whose end feet gets attached to the pia. Neurons reach the cortical plate as the soma is gradually pulled towards the leading edge by shortening the process. Migrating cells might develop the radial process by themselves (Nadarajah et al., 2001a) or inherit it from the RGC by asymmetric division (Miyata et al., 2001) (Fig.2 A).

At later stages of corticogenesis, projection neurons predominantly migrate by locomotion or gliaguided migration, where the RGCs serve as the scaffold that bring them closer to their destined cortical location (Fig.2 B). An elegant study based on time-lapse imaging revealed that these neurons exhibit four phases of migration (Noctor et al., 2004). At phase one, newly-born pyramidal neurons adopt a bipolar morphology and quickly depart from the VZ to the SVZ. Phase two comprises a migratory arrest (for about 24 hours) in the SVZ, and a variation at the morphological level, as neurons acquire a multipolar shape. During phase three, neurons return to a bipolar morphology, extend a leading process and move the cell body towards the apical surface of the VZ. In the course of phase four, cells reverse polarity and adopt a bipolar configuration, with a long leading process at the front and a short trailing process at the rear, typical of migrating neurons. Pia-directed migrating neurons (destined to LV, LIV or LII/III) migrate then along radial glial fibers towards the pial surface. Upon contacting the $M Z$, glia-guided neurons detach from the glial scaffold. Cells then reach their final destination by switching to the 
somal or terminal translocation mode, where neurons pull their somas by shortening the length of their leading process (Nadarajah et al., 2001a) (Fig.2 B).

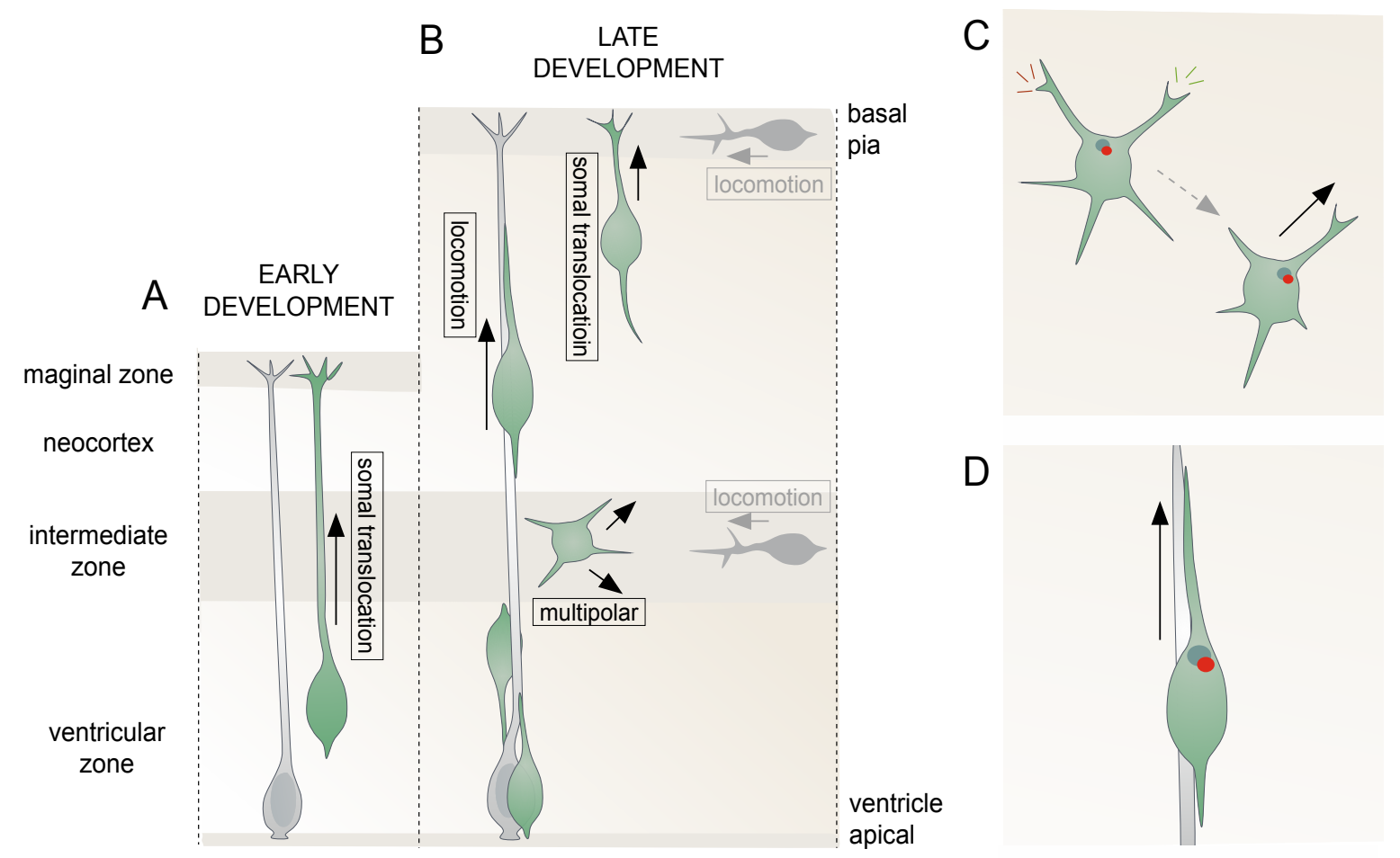

Figure 2. Neuronal migration during brain development in rodents. (A and B) Main strategies of neuronal migration: radial (parallel to the RG fibers), or tangential (orthogonal to the RG fibers) migration, adopted by excitatory (in green) and inhibitory (in dark grey) neurons respectively. (A) At early stages of development neurons preferentially migrate by glia-independent somal translocation. (B) Successive cohorts of pyramidal neurons migrate to the cortex by multipolar, glia-guided locomotion and somal translocation. Interneurons reach the cortex through the intermediate zone or the marginal zone by locomotion. ( $C$ and $D$ ) Neuronal polarization and leading process dynamics. (C) Multipolar neurons use their multiple processes as guiding sensors. At a given time, a branch is stabilized and the dominant neurite recruits the centrosome (in blue) and the Golgi apparatus (in red). The stabilized branch will lead the direction of movement. (D) Neurons that migrate by locomotion are typically bipolar, with an unbranched leading process. The centrosome and Golgi reside basally in the dominant neurite with the nucleus following behind. 


\section{Neuronal movement and polarity}

Although neurons make use of distinct modes of migration, they seem to share a general basic three-step model for neuronal movement: cells initially elongate a leading neurite, the nucleus next translocates into the leading process (a step known as nucleokinesis), and finally the trailing process retracts (Komuro and Rakic, 1995; Wichterle et al., 1997). During nucleokinesis, a dilation forms at the basal end of the leading neurite, in front of the nucleus (Bellion et al., 2005). The centrosome, accompanied by other organelles (for instance the Golgi apparatus), moves to the dilation. The nucleus follows centrosome, being then relocated into the dominant process.

As they migrate along numerous substrates, neurons show a high polarization towards the direction of movement. The direction is headed by the leading process of a given migrating neuron. Bipolar (prospective pyramidal) neurons often have a long leading process in the front, tipped by a highly dynamic structures similar to a dendritic or an axonal growth cone, which select the direction of movement by sensing the chemotactic cues in the surroundings. However, the morphology of the leading process differs during migration as an adaption to the requirements en route. A highly dynamic and unstable leading process, with branches rising and collapsing, e.g. multipolar cells in the SVZ (Tabata and Nakajima, 2003) or interneurons (Bellion et al., 2005), may ease route finding. Multiple growth cones from several branches may sense attractants or repellants in the local or long-range environment. After comparing concentrations, the branch whose cone is closer to the chemoattractant source is selectively stabilized, whereas the others are suppressed. The centrosome and the Golgi are then translocated to the dominant process, which leads to the movement of the nucleus (Cooper, 2013) (Fig.2 C and D).

\section{THE REELER MOUSE:}

\section{A mutant that questions the importance of cortical lamination}

The reeler mouse strain has been essential in the progress of understanding the development of the brain. Reeler refers to an autosomal mutation in the mouse that implies brain development deficiencies. The first documented reeler mutation, referred as the $\mathrm{rl}^{\mathrm{Ed}}$ strain (nowadays designated Rel $\left.n^{r l}\right)$, appeared spontaneously in an inbred colony in Edinburgh, United Kingdom. Mice carrying the reeler mutation were harshly ataxic and presented a distinctive reeling gait, from which they awarded their name (Falconer, 1951). The reeler phenotype is based on a homozygous loss-of-function of the reelin gene, due to large gene deletions or exon-exclusions. 
Animals are consequently null for a functional Reelin protein expression (D'Arcangelo et al., 1995). Histological studies described multiple disruptions in the reeler brain. Thus, reeler mice show a reduction of the total brain volume, increased ventricular size (Badea et al., 2007), significant atrophy of the cerebellum (Mariani et al., 1977) (hence the ataxic gait) and, its most characteristic feature, an abnormal cytoarchitecture of laminated structures, including the cerebellum, the hippocampus and, obviously, the neocortex. However, all major cortical structures of the brain are present (Caviness, 1976) and deficient mice display a surprisingly normal behavior compared to their wild type counterparts (Guy and Staiger, 2017). The Shaking Rat Kawasaki (SRK) and the Komeda Zucker Creeping (KZC) rats show all of the typical anatomical features of the mouse reeler mutation (Aikawa et al., 1988; Yokoi et al., 2003). These animals are particular used for behavioral and pharmacological studies, for which the rat is the preferred species.

All main cohorts of projection neurons are produced on schedule (Caviness and Sidman, 1973), but fail to aggregate in appropriate layers in all laminated brain regions in reeler. Although the preplate forms normally, it is believed that projection neurons fail to split it and accumulate beneath it. The preplate then remains as an unsplitted layer located above the cortical plate during development, called superplate (Caviness, 1982a; Pinto Lord and Caviness, 1979; Sheppard and Pearlman, 1997). Neocortical radial glia scaffold is also disrupted, as radial fibers are shown to have a slightly oblique orientation and a severely reduced branching (Chai et al., 2015; Derer, 1979; Hartfuss et al., 2003; Hunter-Schaedle, 1997). Neurons in reeler seem to maintain an abnormal tight association with glial fibers, an observation that led to the hypothesis that migration of younger neurons is prevented in reeler by older neurons trapped on radial fibers (Pinto Lord and Caviness, 1979). A decisive cell birth dating experiment carried out by Caviness in 1982 showed that, in the cortex of the mutant mouse, early-born neurons are located in upper layers while late-born neurons occupy lower cortical levels, leading to the conclusion that the reeler cortex is shaped in an inverted or outside-in fashion (Caviness, 1982a). The description of the reeler cortex as inverted has been broadly used by the scientific community and, with minor exceptions (Boyle et al., 2011; Dekimoto et al., 2010; Wagener et al., 2010), still prevails nowadays (Chai and Frotscher, 2016; Cooper, 2008; Gupta et al., 2002; Jiang and Nardelli, 2016; Tissir et al., 2002).

It has been also reported that apical dendrites of cortical neurons point downwards, which results in inverted or oblique pyramidal neurons (Goffinet, 1979; Terashima et al., 1985). Extraordinarily, despite the ectopic neuronal location and the morphological abnormalities, afferent projections 
find their correct target cells. Thus, organization and functionality of major neuronal circuits are preserved in reeler mice (Caviness, 1976; Guy et al., 2017; Pielecka-Fortuna et al., 2014; Silva et al., 1991; Wagener et al., 2010; Wagener et al., 2016) .

Although the reeler phenotype has been studied for more than 50 years, it still puzzles neurodevelopmental biologists (D'Arcangelo, 2014). Reelin-deficient mice have been widely used to explore the Reelin-signaling pathway and its roles during cortical development (D'Arcangelo, 2005; Rice and Curran, 2001), but no model proposed so far covers its full complexity. Additionally, these models often combined results from different brain structures (cortex, hippocampus, cerebellum and spinal cord), whose development and cytoarchitectural organization highly differ. What is more, studies focusing on radial neuronal migration in the reeler neocortex often lack brain coordinates. Thus, in these studies the cortex is just referred as neocortex, and the intricacies of its sub-regions (for instance, sensory areas) are not considered. These sub-regions participate in different cognitive processes, happen to have unique developmental times and might regulate equivalent processes differently.

\section{REELIN AS THE GUIDE FOR RADIAL NEURONAL MIGRATION}

Radial migration of excitatory neurons is precisely regulated by a large number of signaling pathways, mainly driven by secreted extracellular molecules (Ohtaka-Maruyama and Okado, 2015). Among these, the large extracellular matrix glycoprotein Reelin stands unquestionably as the key player (Marin et al., 2010).

\section{The Reelin protein}

Reelin is encoded by the reelin (Reln) gene (D'Arcangelo et al., 1995). The full-length protein has a relative molecular mass of about $385 \mathrm{kDa}$ in mice and, at the amino acid level, is $95 \%$ identical to the protein found in humans. The protein consists of three subdomains: a) an N-terminal, which comprises the signal peptide and has a sequence comparable to F-spondin, b) eight consecutive Reelin repeats centered around an EGF (epidermal growth factor)-like cysteine motif and c) a short C-terminal, positively charged, required for Reelin secretion (D'Arcangelo et al., 1997). After secretion, Reelin is subjected to enzymatic cleavage in vivo (Lambert de Rouvroit et al., 1999), producing different fragments of various lengths and diffusion properties. While the full-length protein is mainly located in the marginal zone, the diffusion of smaller fragments throughout the 
cortical wall indicates its participation in processes away from the secretion site (Jossin et al., 2007).

\section{Reelin expression in the brain}

During embryonic forebrain and hindbrain development, Reelin is abundantly expressed and secreted by Cajal Retzius cells located in the marginal zone of the neocortex and hippocampus (Alcántara et al., 1998; D'Arcangelo et al., 1995; Ogawa et al., 1995), and by granule cells of the external granule layer in the cerebellum (Alcántara et al., 1998; Miyata et al., 1996). Cajal Retzius cells die shortly after birth, according to the end of neuronal migration and cortical lamination, causing a gradual reduction of Reelin from the marginal zone. In the postnatal and adult forebrain, inhibitory GABAergic interneurons slowly become a predominant and long-lasting source of Reelin (D'Arcangelo, 2014; Pohlkamp et al., 2014). An abnormal expression of Reelin has been associated to several neurodevelopmental disorders. For a review see (Folsom and Fatemi, 2013)

\section{Reelin receptors}

The best described receptors in the Reelin signaling cascade are ApoER2 (apolipoprotein E receptor type 2, also known as Lrp8) and VLDLR (very low density lipoprotein receptor) (D'Arcangelo et al., 1999; Trommsdorff et al., 1999). The intracellular domains of these lipoprotein receptor family members bind to a diversity of proteins associated with kinase signaling, cytoskeletal reorganization, cell adhesion and synaptic transmission (Gotthardt et al., 2000). The Reelin signal transduction is dependent on the presence of Dab1 (Disabled-1). Dab1 is an intracellular adaptor protein that binds to the cytoplasmic domains of ApoER2 and VIdlr. Upon Reelin binding, Dab1 is phosphorylated on tyrosine residues by Src family kinases (Hiesberger et al., 1999; Howell et al., 1999), triggering an intracellular cascade that involves actin and microtubule remodeling, crucial for neuronal migration. The initial analysis of single and double apoer2 and vldlr knock out mice suggested a coordinated but partially overlapping function. The phenotypes were, however, slightly different. Malformations in $\mathrm{ldll}^{-/}$mice were predominantly found in the cerebellum while in apoer $2^{-/}$animals deficiencies were mainly seen in the neocortex and the hippocampus. Moreover, although neurons invaded the marginal zone, the layering of $v / d l r^{-}$mice was largely normal; on the contrary, the cortical lamination of apoer $2^{-}$mice was severely affected, producing a partial inversion (Trommsdorff et al., 1999) . Further evidence indicates that these receptors play different roles in migrating neurons. Biochemical studies have 
shown that Reelin binds more easily to ApoER2 and the level of Dab1 phosphorylation induced by Reelin is higher in neurons deficient for ApoER2 in comparison with cells lacking VLDLR (Andersen et al., 2003; Benhayon et al., 2003). Moreover, VLDLR is mainly found in the distal parts of the leading processes of migrating neurons in contact with the marginal zone, while ApoER2 is preferentially located in the neuronal processes of multipolar cells in the SVZ (Hirota et al., 2014) in the developing neocortex in mice, thus indicating divergent roles.

Integrins were also proposed as Reelin receptors. Binding experiments associated $\alpha 3 / \beta 1$ and $\alpha 3 / \beta 5$ integrins to Reelin in migrating cortical neurons in vitro (Dulabon et al., 2000). Although the finding could not be replicated by others, new evidence indicates that the role of integrins in the Reelin signaling cascade should not be rejected, but re-especified. The exploration of the hippocampus (Forster et al., 2002) and knockouts for several integrins (Graus-Porta et al., 2001) indicate that $\beta 1$ class integrins are rather necessary for the stability of the radial glial fibers. Reelin has been shown to modify the integrin-dependent adhesive properties of radially migrating neurons in the cortical plate (Sanada et al., 2004) and to stimulate the attachment of their leading process to the marginal zone (Sekine et al., 2012). Thus, the collaboration between $\beta 1$ integrins and the Reelin signaling cascade is likely to contribute to layer formation mainly by their influence on the radial glial scaffold.

Cadherin-related neuronal receptor (CNR) and Ephrin B (EphB) family proteins have been also proposed to operate as Reelin receptors. CNR and EphB were reported to bind to the Nterminal section of Reelin (Senturk et al., 2011; Senzaki et al., 1999). Nonetheless, the findings for CNR could not be replicated and the significance of the Ebf2-Reelin interaction was shown to be rather mild.

Taken together, the experimental data available so far support the concept that ApoER2 and VLDLR are the main receptors for Reelin and thus, though their specific roles are still under debate, predominantly mediate its activity in the developing cortex.

\section{Biological roles of Reelin in neuronal migration}

The fact that the expression of Reelin is spatiotemporally regulated highlights its multiple roles during the development of the brain. Reelin regulates neuronal migration and cell positioning during development, promotes dendrite outgrowth and spine formation postnatally and modulates synaptic transmission during adulthood. Due to its relevance to the project, only neuronal migration and cell positioning with regard to neocortical projection neurons will be 
further detailed. For a review about Reelin's contribution to spine formation and synaptic function see (D'Arcangelo, 2014). Functional connectivity in Reelin-deficient mice has been evaluated by us recently (Guy et al., 2017; Wagener et al., 2010; Wagener et al., 2016).

\section{Early stages of corticogenesis: Reelin as a permissive cue}

Early-born neurons split the preplate by somal translocation, which implies the development of a long leading process, used as an anchor to pull the soma. As LVI cells are unable to divide the preplate in reeler mice (Sheppard and Pearlman, 1997) and leading processes of neurons Dab1deficient end beneath the preplate (Sanada et al., 2004), Reelin was proposed as an indispensable protein for early-born neurons to conquer the preplate. Reelin has also been shown to induce branching and elongation of glial fibers in the cortex and hippocampus (Chai et al., 2015; Zhao et al., 2004) and causes glial scaffold disruption when absent (Derer, 1979; Weiss et al., 2003). These observations lead to the conclusion that Reelin may sponsor the migration of early-born neurons by instructing the branching and anchoring of the leading processes in the marginal zone, facilitating then the translocation of the cell body. Two additional experiments supported the hypothesis of Reelin as a permissive signal at the beginning of the corticogenesis: neurons in reeler do split the preplate when Reelin is expressed ectopically in the VZ in vivo and when incubated with a recombinant full-length Reelin protein in vitro (Jossin et al., 2004; Magdaleno et al., 2002). Laminar organization could not be rescue, though.

\section{Late stages of corticogenesis: multiple roles of Reelin}

The cytoarchitecture of the reeler cortex and the consequences of Dab1 loss-offunction in migrating neurons, led to consider Reelin as an attractive factor. In wild type animals, neurons migrate past their predecessors, aiming for the Reelin-containing marginal zone, while in the absence of Reelin (e.g. in reeler) late-born neurons fail to bypass their predecessors, accumulate at lower cortical levels and develop aberrantly oriented dendrites (Tabata and Nakajima, 2002). On the other hand, Dab1 loss-of-function or the expression a mutated form of Dab1 elicit a reduction in migration speed and leading process development (Olson et al., 2006; Sanada et al., 2004). Additionally, the fact that cortical lamination could not be rescued (Jossin et al., 2004) (Magdaleno et al., 2002), speaks in favor of a Reelin gradient that attracts neurons to the Reelin-rich zone. It was also proposed that Reelin may exert its attractive effect on migrating neurons through the RGCs, as they express the main Reelin receptors. However, this is rather unlikely, as Dab1-deficient radial glial fibers does not inhibit neuronal migration (Sanada et al., 2004). 
Several observations led to consider Reelin also as a stop signal. For instance, the marginal zone of wild type mice, where Reelin is highly concentrated, is rather cell-sparse but it is highly colonized in reeler. A similar scenario was found when CR cells were depleted, as cells invaded the marginal zone (Super et al., 2000). Moreover, real-time time imaging showed that cortical neurons terminate migration upon contacting the Reelin-rich zone (Dulabon et al., 2000). However, when Magdaleno and colleagues expressed Reelin in the developing cortex, it did not stop migration in the first place (Magdaleno et al., 2002).

It has been also described that neurons in Scrambler (Dab1-deficient) and reeler mice are abnormally associated with glial fibers (Pinto Lord and Caviness, 1979; Sanada et al., 2004), thus indicating that Reelin might function as a detachment cue. In support of this conjecture, aggregation assays showed that cortical neurons in reeler are highly adhesive and cultured neurons seemed to detach from the glia after Reelin treatment in vitro (Hack et al., 2002; Hoffarth et al., 1995).

Reelin has been also proposed as a cytoskeleton stabilizer. It appears that Reelin inhibits migration and stabilizes actin in the cytoskeleton by Dab-1 induced phosphorylation of Cofilin (an actin-associated protein) in the leading edge of migrating neurons (Chai et al., 2009). Components of the microtubule-associated proteins, such as Tau or Lis, have been also suggested as Reelin targets indicating its potential role in assembling the cytoskeleton (Assadi et al., 2003; Beffert et al., 2002).

In summary, Reelin stands undeniably as a positional cue and a key regulator for cortical lamination. It participates in these processes at various levels, as RGCs and migrating neurons at any given location of the cortical wall are subjected to Reelin's action. However, many aspects of the Reelin-signaling pathway remain elusive: different hypotheses are based on partially contradictory results and its specific roles on distinctive cohorts of migrating neurons are waiting to be resolved. 


\section{AIMS AND APPROACHES}

Precise neocortical function seems to be dependent on an accurate lamination. The development of the horizontal layers relies on an appropriate cell production, migration and positioning during embryonic and early postnatal stages. The large extracellular matrix glycoprotein Reelin, encoded by the reelin (Reln) gene (D'Arcangelo et al., 1995), plays a key role in orchestrating these processes. The reeler mouse strain (Falconer, 1951) is a natural mutant lacking Reelin and famous for a pretended inverted cortical lamination. This mutant mouse has been essential in the progress of understanding the development of the brain and has directed the hypothesis about Reelin's role. However, diverse models of Reelin's action, influenced by an incomplete description of the reeler phenotype, have been proposed and still remain highly debatable (Chai and Frotscher, 2016; D'Arcangelo and Curran, 1998; Tissir and Goffinet, 2003). Thus, this PhD project was focused on elucidating some controversial aspects of Reelin functioning and the reeler phenotype. To achieve that goal, we investigated the progress of neuronal migration, cell positioning and cortical lamination in the reeler mouse, by using a combination of demanding techniques such as RNA in situ hybridization, novel (cell) birth dating approaches and in utero electroporation.

Previous studies from the group have shown that, at least in the reeler somatosensory cortex, LII/III, LIV, and LV cells are severely intermingled rather than inverted (Wagener et al., 2010). Thus, we wanted to determine first whether the cortex is indeed inverted along the entire rostro-caudal axis of the reeler brain. Lamination of the adult cerebral cortex was then examined in detail in the main primary cortices, conveniently located over the anterior-posterior axis, by visualizing the expression of layer-fate markers. Next, and based on the results from those makers, it was evaluated the presence of the superplate, one of the main features used to explain the cortical inversion in reeler. The analysis of a uniform existence of the superplate was carried out by the detection of its main cell components by double in situ hybridization and by inspecting the trajectories of thalamocortical projections, in rostral and caudal regions, at early stages of development.

Although the effects of Reelin on the cortical structure have been extensively analyzed over the last decades, the view that neurons are distributed in an outside-in fashion in reeler still rests on experiments done by Caviness more than 50 years ago, with very simple and only partially conclusive techniques (Caviness, 1982a; Caviness and Sidman, 1973). Moreover, the analysis of cell production and cell positioning is surely necessary when facing a mouse mutant that defies 
the assembling of a normal layered cortex. Thereby, area-specific neocortical histogenesis was traced over development by making use of a novel cell birth dating approach. This approach combines three different types of thymidine analogs, allowing a distinct labeling of infragranular, granular- and supragranular-fated neurons on the same tissue. It hence provides an excellent tool to study cortical lamination during development and its final shape at postnatal stages.

Additionally, as how cohorts of projection neurons respond to the lack of Reelin remains still vague, migratory kinetics of early- and late- born neurons were further evaluated, in rostral and caudal cortices of reeler mice, by making use of the FlashTag technique (Telley et al., 2016). This technique ensured outstanding and highly reliable tracing of VZ-derived progeny over development, which was of a great value to study where deficiencies in migrating neurons started, and whether those could be discriminated in an area- and cohort- dependent fashion. Moreover, the pillars of neuronal migration, polarity and morphology, were investigated in in utero electroporated and Flash Tagged late-born neurons, from the exit of the ventricular until their final presumable cortical location, in anterior and posterior cortices of Reelin-deficient mice.

This PhD project also reserved a slot for the main Reelin receptors. Various roles for ApoER2 and VLDLR have been suggested, but its specific contribution to each step of neuronal migration remain debatable. Thus, as an attempt to discriminate potential roles of these receptors during early stages of migration, projection neurons were transfected with ApoER2 and VLDLR (subcloned de novo) expression plasmids by in utero electroporation and analyzed shortly after. 


\section{MATERIALS AND METHODS}

\section{Animals}

B6C3Fe wild type, B6C3Fe rl-/- (known as reeler), CD1 IGS and ROR ${ }^{\text {tdTomato }}$ mice were used for the experiments. The reeler mice colony, obtained from the Jackson Laboratory (Ref.\#000235), (D'Arcangelo and Curran, 1998) was maintained by mating heterozygous males and females and genotyped as described in (Wagener et al., 2016). Time-mate CD1 IGS mice were obtained from The Charles River Laboratories. The ROR ${ }^{\text {tdTomato }}$ line was generated as follows: ROSA-Tomato-LSL mice (full strain name: B6; 129S6-Gt (ROSA)26Sortm9(CAG-tdTomato)Hze/J; Ref.:\#007905; Jackson Laboratory) were crossed with heterozygous reeler mice. The resulting ROSA-Tomato-LSL reeler mice were crossed again with each other, and animals homozygous for ROSA-Tomato-LSL and heterozygous for Reelin were saved for further crossings. The ROR $\alpha$-IRES-Cre line (Chou et al., 2013) was crossed in the same way with heterozygous reelers, leading to the ROR $\alpha-I R E S-C r e$ reeler line. Crossing heterozygous ROSA-Tomato-LSL reeler and ROR $\alpha$-IRES -Cre reeler resulted in the expression of tdTomato in ROR $\alpha$ expressing cells in animals with both wild type and reeler background. Thus, this line was named ROR $\alpha^{\text {tdTomato }}$.

Mice were housed with a 12-h light/dark cycle, with food and water available ad libitum, at the central animal facility of the University Medical Center Goettingen. All experimental procedures were performed in accordance with the European Guidelines for the Care and Use of Laboratory Animals and approved by the local government. The day of vaginal plug detection (at noon) was considered embryonic day 0.5 (E0.5). The day of birth was defined as postnatal day 0 (PO).

\section{Fixation and Tissue Processing}

Adult animals were perfused transcardially with $10 \mathrm{ml}$ of $10 \%$ sucrose, followed by $200 \mathrm{ml}$ of $4 \%$ paraformaldehyde (PFA) in $0.1 \mathrm{M}$ phosphate buffer (PB) pH 7.4. Brains were dissected out of the skull, post-fixed for $2 \mathrm{~h}$ in $4 \%$ PFA, and then cryoprotected in $20 \%$ sucrose in $0.01 \mathrm{M}$ phosphatebuffered saline (PBS) overnight at $4^{\circ} \mathrm{C}$. Free-floating coronal sectioning was done with a cryostat (CM 3050S; Leica) at $40 \mu \mathrm{m}$ thickness in 10mM PBS (dissolved in DEPC-treated $\mathrm{H}_{2} \mathrm{O}$ ). 
Embryonic and early postnatal brains (E13.5-P7) were dissected in cold DEPC-treated $\mathrm{H}_{2} \mathrm{O}$, immersion- fixed in 4\% PFA in 0.1 M PB pH 7.4 for 3 h (E13.5, whole head), 2 h (E15.5, from E15.5 on just the brain), $2.5 \mathrm{~h}$ (E17.5), $3.5 \mathrm{~h}$ (P0) or $5.5 \mathrm{~h}$ (P7) and dehydrated in $25 \%$ sucrose in $0.01 \mathrm{M}$ PBS overnight at $4^{\circ} \mathrm{C}$. Fixed tissue was then embedded and cryopreserved in O.C.T. compound (Tissue-Tek), coronally sectioned with a cryostat at $14 \mu \mathrm{m}$ thickness and stored at $-20^{\circ} \mathrm{C}$ until use.

\section{Fluorescent Immunostaining}

After the sections were rinsed in $0.01 \mathrm{M}$ PBS $(2 \times 15 \mathrm{~min})$, non-specific antigenic sites were blocked with $10 \%$ normal goat serum in PBS $0.1 \%$ Triton X-100 (blocking buffer) for 1 hour. Tissue was immunostained overnight at $4^{\circ} \mathrm{C}$ in primary antibody diluted into blocking buffer.

The primary antibodies and dilutions were used as follows: rabbit anti- Calretinin (1:2000; Swant, CR7699/3H), rabbit anti-RFP (1:2000; Rockland, 600-401-379), rat anti-BrdU (CldU-1:250; Abcam, ab6326), mouse anti-BrdU IgG1 (IdU- 1:200; BD, 347580), rabbit anti-Trb1 (1:300; Abcam, ab31940), rabbit anti-Cux1 (1:100; Santa Cruz Biotechnology, sc13024), rat anti-Ctip2 (1:300; Abcam, ab18465), mouse anti-Pax6 IgG1 (1:300; DSHB), Tbr2 (1:500; Abcam, ab23345), rabbit anti-GFP (1:2500; Invitrogen, A11122), rabbit anti-Fluorescein (1:1000; Abcam, ab19591), mouse anti-GM130 IgG1 (1:100; BD Biosciences, 610823), mouse anti-Stab2 IgG1 (1:200; Abcam, ab51502) and guinea pig anti-Glast (1:200; Frontier Science Co.Ltd, Af1000-1), reelin (clone G10, 1:100 Goffinet's laboratory). In order to detect nucleosid analogues like 5-bromo-2'-deoxyuridine (BrdU), 5-iodo-2'-deoxyuridine (IdU) and/or 5-chloro-2'-deoxyuridine (CldU), a pretreatment, with $2 \mathrm{~N} \mathrm{HCl}$ for $30 \mathrm{~min}$ at $37^{\circ} \mathrm{C}$ was applied to retrieve the antigen before the incubation with the primary antibodies. The subsequent day, sections were rinsed and then incubated for 2 hours with the appropriate fluorescent conjugated secondary antibodies, diluted in blocking buffer. The following secondary antibodies were used (dilution 1:500): goat anti-rabbit Alexa Fluor 568 (Invitrogen, A11035), goat anti-rat Alexa Fluor 488 (Invitrogen, A11006), goat anti-mouse IgG1 Alexa Fluor 568 (Invitrogen, A21124), goat anti-rabbit Alexa Fluor 488 (Invitrogen, A11034); goat anti-rat Alexa Fluor 647 (Invitrogen, A21247), goat anti-rat Alexa Fluor 647 (Invitrogen, A21247), goat anti-guinea pig Alexa Fluor 633 (Invitrogen, A21105). Detection of 5-Ethynyl-2'-deoxyuridine (EdU) was performed according to the manufacturer's instructions (Click-IT Plus EdU Alexa Fluor 647 Imaging Kit, ThermoFisher cat.C10640). 
Nuclei were counterstained with 4' 6-diamidino-2-phenylindole (DAPI) (1:1000, Molecular Probes). Double and triple immunofluorescence stainings were performed sequentially (one antigen after another). Finally, sections were mounted and coverslipped with Aqua-Poly/Mount (Polyscience, 18606), Fluoromount (Sigma, F4680) or Prolong Gold (Invitrogen, P36930) according to the requirements of the experiment.

\section{In situ hybridization}

\section{Probe generation}

In Situ Hybridization (ISH) for: a) layer-specific markers Ndnf (also known as A930038C07Rik), Rgs8, Rorb (also known as Rorbeta), Etv1 (also known as Er81), and $T C_{1460681}$, and b) preplatespecific genes Trp73, Ebf2, Cdh10, Cdh18, Gabra5, Unc5d and Pls3 were performed with, sense and antisense single-strand, fluorescein isothiocyanate (FITC)- or digoxigenin (DIG)-labeled cRNA probes.

Riboprobes were either produced directly from the plasmids containing the full-length cDNA inserts specific to the particular gene or amplified from genomic cDNA. Catalog numbers of the full-length plasmids (Source BioScience or Genome Cube) were: Ndnf (IRAVp968E1091D), Rgs8 (IRAVp968E09162D), Rorb (IRAVp968D1267D), Etv1 (IRAKp961E106Q), Trp73 (IRAVp968H12129D). As for $T C_{1460681,}$ Cdh10, Cdh18, PIs3, Unc5d, Ebf2, probes were synthesized from genomic cDNA. Total RNA of E15.5 or adult cortex was extracted by following the manufacturer's instructions (Quiagen RNAeasay Mini Plus Kit, Cat.74134) and cDNA was synthesized by using the Super Script II Reverse Transcriptase kit (TermoFisher, Cat.18064022), following the protocol provided by the company. After primer validation by using a temperature gradient specific for the primer pair (Quiagen Taq DNA fel 201207), DNA fragments were amplified with the gene-specific forward and reverse primers listed in Table 2, using the KOD Hot Start DNA Polymerase Amplification Kit (Millipore, Cat.71086-3). The PCR products were loaded in a $2 \%$ agarose gel and electrophoretically separated, purified (NucleoSpin ${ }^{\circ}$ Gel and PCR Clean-up kit, Macherey-Nagel, Cat. 740609.240C) and then ligated into the pCR-Blunt II-TOPO plasmid (Zero Blunt TOPO PCR Cloning Kit, Invitrogen, 45-0245 ).

Plasmids were transformed in JM109 competent E.coli (Promega, Cat. L2005) by heat shock. Briefly, thawed vials (on ice) of competent E.coli cells and ligated plasmids were incubated for 20 min on ice and heat shocked at $42^{\circ} \mathrm{C}$ for 50 secs. Vials were next transferred to ice for 2 minutes 
and bacteria were left to recover in S.O.C. medium (ThermoFisher, Cat. 15544034) for $90 \mathrm{~min}$ at $37^{\circ} \mathrm{C}$ at $150 \mathrm{rpm}$. After mixing and diluting in LB medium supplemented with $50 \mu \mathrm{g} / \mathrm{ml} \mathrm{Kanamycin}$, each dilution was spread onto a Kanamycin selection plate, which were incubated overnight at $37^{\circ} \mathrm{C}$. Bacterial cultures from single colonies were amplified overnight in Luria-Bertani (LB) medium or LB medium supplemented with $50 \mu \mathrm{g} / \mathrm{ml}$ Kanamycin and purified with the QIAprep Spin Miniprep Kit (Quiagen, Cat. 27106). Plasmids were afterwards digested with the appropriate enzymes, separated in electrophoresis (in 1.5\% agarose gel) purified and sent for sequencing. Colonies were selected based on the sequencing results, LB-supplemented medium was inoculated with the validated single-colony cultures, amplified overnight (at $37^{\circ} \mathrm{C}$ at $150 \mathrm{rpm}$ ) and purified using the Quiagen Plasmid Maxi Kit (Cat.12163). Plasmids underwent restriction digestion with the suitable enzymes, and linearized products were loaded in a 1.5\% agarose gel and purified subsequently (NucleoSpin ${ }^{\circledR}$ Gel and PCR Clean-up kit, Macherey-Nagel, Cat. 740609.240C).

The following steps, until the stringency washes, were performed RNAse-free. All solutions were made with $0.1 \%$ diethyl pyrocarbonate (DEPC)-treated water and instruments baked at $55^{\circ} \mathrm{C}$ for 5 hours, or bought RNase-free. Antisense and sense (negative control) complementary RNA (CRNA) probes were in vitro transcribed using either SP6 (Roche, 1081274001) or T7 (Roche, 10881767001) RNA polymerases with digoxigenin (DIG)- (Roche, 11277073910) or fluorescein (FITC)- (Roche, 11685619910) labeled nucleotides. If sizes of the full-length probes were longer than $1000 \mathrm{pb}$, riboprobes were reduced to around 350 base pairs via alkaline hydrolysis by incubating the probes in $0.2 \mathrm{M}$ sodium carbonate and $0.2 \mathrm{M}$ sodium bicarbonate at $\mathrm{pH} 10.2$ at $60^{\circ} \mathrm{C}$ for a probe-specific time by using the following formula: $\mathrm{t}(\mathrm{min})=$ (probe length $(\mathrm{Kb})$-desired length $(\mathrm{Kb})) /(0.11 \times$ probe length $(\mathrm{Kb}) \mathrm{x}$ desired length $(\mathrm{Kb}))$. RNA was then precipitated (at $-80^{\circ} \mathrm{C}$ for $30 \mathrm{~min}$ ) by adding $4 \mathrm{M} \mathrm{LiCl}$ and $100 \% \mathrm{EtOH}$ to the product of the in vitro transcription (or alkaline hydrolysis). Air-dried pellets were dissolved in DEPC-treated water and RNA concentration and quality was measured by using a Qubit 2.0 Fluorometer (ThermoFisher Scientific, Q32866). Probes were stored at $-80^{\circ} \mathrm{C}$ until use.

\section{Free-floating in situ hybridization (ISH) (adult tissue)}

In situ hybridization was performed as previously described (Wagener et al., 2016). Sections were washed 3 times with $2 \times$ standard saline citrate $(1 \times \mathrm{SSC}: 0.15 \mathrm{M} \mathrm{NaCl}, 0.015 \mathrm{M}$ sodium citrate, $\mathrm{pH}$ 7.0) and pretreated for $15 \mathrm{~min}$ in hybridization buffer (HB; $50 \%$ formamide, $4 \times \mathrm{SSC}, 250 \mu \mathrm{g} / \mathrm{ml}$ 
denaturated salmon sperm DNA, $100 \mu \mathrm{g} / \mathrm{ml}$ tRNA, 5\% dextransulfate and 1\% Denhardt's solution) diluted $1: 1$ in $2 \times S S C$ at room temperature. Slices were next incubated $1 \mathrm{~h}$ in pure $\mathrm{HB}$ at $55^{\circ} \mathrm{C}$ and hybridized, overnight at $55^{\circ} \mathrm{C}$, with $200 \mathrm{ng} / \mathrm{ml} \mathrm{DIG-labeled} \mathrm{probes} \mathrm{in} \mathrm{HB}$. Stringency washes: $2 \times \mathrm{SSC}$ ( $2 \times 15 \mathrm{~min}$, at room temperature), $2 \times \mathrm{SSC} / 50 \%$ formamide ( $15 \mathrm{~min}$, at $65^{\circ} \mathrm{C}$ ), $0.1 \times \mathrm{SSC} / 50 \%$ formamide $\left(15 \mathrm{~min}\right.$, at $\left.65^{\circ} \mathrm{C}\right), 0.1 \times \mathrm{SSC}\left(2 \times 15 \mathrm{~min}\right.$, at $\left.65^{\circ} \mathrm{C}\right)$ and $0.01 \mathrm{M} \mathrm{TBS}, \mathrm{pH} 7.4(2 \times 10 \mathrm{~min}$, at room temperature). After the blocking treatment in 1\% blocking reagent (Roche, 11096176001 diluted in TBS $\mathrm{pH} 7.5$ ), sections were incubated overnight at $4^{\circ} \mathrm{C}$ with anti-DIG Fab fragments conjugated to alkaline phosphatase (Roche, sheep antibody, 11093274910) diluted 1:1500 in blocking buffer. The tissue was next incubated with nitroblue tetrazolium and 5-bromo-4chloro3-indolylphosphate (NBT/BCITP, Roche 11383213001, 11383221001) in buffer composed of 100 $\mathrm{mM}$ Tris- $\mathrm{HCl}, 100 \mathrm{mM} \mathrm{NaCl} \mathrm{pH} 9.5$ and $50 \mathrm{mM} \mathrm{MgCl2}$, which forms a precipitate after reacting with the alkaline phosphatase. Development of the staining reaction was monitored at regular intervals with a stereo microscope. Once the desired intensity of the chromogenic signal was reached, sections were rinsed in TBS and mounted in Kaiser's glycerol gelatin (Merck, 11092420100).

\section{In situ hybridization on a slide glass (embryonic tissue).}

Chromogenic ISH (CISH). Sections were postfixed with 4\% PFA for 20 min on ice and rinsed in PBS (2x $10 \mathrm{~min}$ ). Endogenous peroxidase activity was quenched with $1 \% \mathrm{H}_{2} \mathrm{O}_{2}$ in methanol for $15 \mathrm{~min}$, rinsed shortly (PBS, $2 \times 2 \mathrm{~min}$ ), deproteinized with $0.2 \mathrm{~N} \mathrm{HCl}$ for $8 \mathrm{~min}$, washed (PBS, $1 \times 2 \mathrm{~min}$ ) and permeabilized for probe penetration with proteinase $\mathrm{K}(20 \mu \mathrm{g} / \mathrm{ml})$ in 0.05M EDTA pH 8, $0.05 \mathrm{M}$ TB $1 \mathrm{M} \mathrm{pH} 7.5$ for $3 \mathrm{~min}$. Slides were then rinsed (PBS, $1 \times 5 \mathrm{~min}$ ), treated with 4\% PFA for $20 \mathrm{~min}$ on ice, and acetylated with $0.2 \%$ acetic anhydride in $0.1 \mathrm{M}$ triethanolamine hydrochloride. Tissue was washed (PBS, $1 \times 5 \mathrm{~min}$; 2xSSC, $1 \times 5 \mathrm{~min}$ ) and dehydrated with ethanol (EtOH) in the following sequence: $30 \%$ EtOH (20 sec), 50\% EtOH (20 sec), 70\% EtOH (1 min), 80\% EtOH (20 sec), 95\% EtOH $(20 \mathrm{sec})$ and $100 \% \mathrm{EtOH}(2 \times 20 \mathrm{sec})$. Sections were next prehybridized for at least $1 \mathrm{~h}$ at $55^{\circ} \mathrm{C}$ in hybridization buffer (HB) and hybridized again in HB with the DIG-labelled probes $(200 \mathrm{ng} / \mathrm{ml})$ overnight at $55^{\circ} \mathrm{C}$. Posthybridization stringency washes: $5 \times \mathrm{SSC}\left(1 \mathrm{~min}\right.$, at $\left.65^{\circ} \mathrm{C}\right), 2 \times \mathrm{SSC} / 50 \%$ formamide $\left(30 \mathrm{~min}\right.$, at $\left.65^{\circ} \mathrm{C}\right), 1 \times \mathrm{SSC} / 50 \%$ formamide $\left(30 \mathrm{~min}\right.$, at $\left.65^{\circ} \mathrm{C}\right), 0.1 \times \mathrm{SSC}\left(30 \mathrm{~min}\right.$, at $\left.65^{\circ} \mathrm{C}\right)$. After being rinsed (TBS pH 7.5, $3 \times 2$ min at $30^{\circ} \mathrm{C}$ ) and blocked with $1 \%$ blocking reagent diluted in TBS pH 7.5 (30 min), sections were incubated with anti-DIG-alkaline phosphatase (AP) antibody 1:500 (Roche,11093274910) in blocking buffer in a humidified chamber overnight at $4^{\circ} \mathrm{C}$. On the 
third day of the experiment, after several washes (TBS pH 7.5, 2x 10 min; reaction buffer $100 \mathrm{mM}$ Tris- $\mathrm{HCl}, 100 \mathrm{mM} \mathrm{NaCl} \mathrm{pH} 9.5$ and $50 \mathrm{mM} \mathrm{MgCl} 2,1 \times 10 \mathrm{~min}$ ), the hybridized probes were visualized by the NBT/BCIP color reaction and coverslipped as described above. As a negative control, some sections were also hybridized with the sense probe and developed in parallel.

Table 1. Genes selected for in situ hybridizations.

\begin{tabular}{|c|c|c|}
\hline Gene & Name & Function \\
\hline$N d n f$ & $\begin{array}{l}\text { Neuron Derived } \\
\text { Neurotrophic Factor } \\
\text { RIKEN cDNA A930038C07 } \\
\text { gene }\end{array}$ & $\begin{array}{l}\text { Cell adhesiveness, neuron migration and neurite outgrowth } \\
\text { promotor (NCBI Gene ID: 68169) }\end{array}$ \\
\hline Rgs8 & $\begin{array}{l}\text { Regulator of G-protein } \\
\text { Signaling } 8\end{array}$ & $\begin{array}{l}\text { Inhibitor of signal transduction in G-protein signaling cascades. } \\
\text { (NCBI Gene ID: 67792) }\end{array}$ \\
\hline Rorb & $\begin{array}{l}\text { RAR-Related Orphan } \\
\text { Receptor B }\end{array}$ & DNA binding protein (NCBI Gene ID: 225998) \\
\hline Etv1 & ETS variant gene 1 & $\begin{array}{l}\text { Regulator of cell growth, migration, proliferation and } \\
\text { differentiation (NCBI Gene ID: 14009) }\end{array}$ \\
\hline$T C_{1460681}$ & $\begin{array}{l}\text { Immunoglobulin heavy } \\
\text { constant }\end{array}$ & $\begin{array}{l}\text { Upon binding to the specific antigen, triggers the expansion and } \\
\text { differentiation of B-lymphocytes (NCB| Gene ID: 16019) }\end{array}$ \\
\hline $\operatorname{Trp73}$ & Tumor Protein P73 & $\begin{array}{l}\text { Involved in cellular responses to development and stress } \\
\text { (NCBI Gene ID: 22062) }\end{array}$ \\
\hline Ebf2 & Early B-Cell Factor 2 & $\begin{array}{l}\text { Transcription factor involved in a variety of developmental } \\
\text { processes (NCBI Gene ID: 13592) }\end{array}$ \\
\hline Pls3 & T-Plastin & $\begin{array}{l}\text { Actin-bundling protein; neurite growth regulator } \\
\text { (NCBI Gene ID: 102866) }\end{array}$ \\
\hline Gabra5 & GABA-A receptor $\alpha 5$ & Ionotropic GABA receptor subunit (NCBI Gene ID: 110886) \\
\hline Cdh10 & Cadherin 10 & Type II cadherin (NCBI Gene ID: 1008) \\
\hline Cdh18 & Cadherin 18 & Type II cadherin (NCBI Gene ID: 320865) \\
\hline Unc5d & Unc homologue $5 d$ & Repulsive netrin-1 receptor (NCB| Gene ID: 210801) \\
\hline Reln & Reelin & $\begin{array}{l}\text { Extracellular protein that controls cell-cell interactions during } \\
\text { brain development (NCBI Gene ID: 14009) }\end{array}$ \\
\hline
\end{tabular}


Fluorescence ISH (FISH). In situ hybridizations were performed as described in the previous section, with the following modifications. On the second day, sections were blocked with $0.5 \%$ blocking reagent (PerkinElmer, FP1012) in TBS pH 7.5 (blocking buffer; $30 \mathrm{~min}$ at $30^{\circ} \mathrm{C}$ ) and incubated with the anti-DIG-POD antibody (1:500, Roche, Ref.11207733910) overnight at $4^{\circ} \mathrm{C}$. After rinsing (TBS pH 7.5, 3x $10 \mathrm{~min}$ ), and aiming for fluorescent detection, TSA-Cyanine 5 (1:50 in amplification diluent) (TSA Plus Cyanine 5 System, PerkinElmer Cat.NEL745001KT) was applied for 10 min. Finally, sections were washed in TBS (pH 7.5, 3x 5 min), counterstained with DAPI (1:1000 diluted in washing buffer) and mounted with Aqua-Polymount.

Dual-probe fluorescence ISH (dFISH). dFISH was performed as explained above with minor adjustments. On the first day, tissue was incubated (overnight at $55^{\circ} \mathrm{C}$ ) with DIG (PIs3)- and FITC (Trp73)- labelled probes $(200 \mathrm{ng} / \mathrm{ml})$, and with the anti-DIG-POD antibody (overnight at $4^{\circ} \mathrm{C}$ ) the following day. On the third day, after rinsing, slides were incubated with TSA-Cyanine 3 (1:50 in amplification diluent) (TSA Plus Cyanine 3 System, PerkinElmer Cat.NEL744001KT) for 10 min. To detect the additional probe, and after rinsing (TBS pH 7.5, 3x $10 \mathrm{~min}$ ), POD was blocked by using $3 \% \mathrm{H}_{2} \mathrm{O}_{2}$ in $\mathrm{PBS}\left(5 \times 2 \mathrm{~min}, 30^{\circ} \mathrm{C}\right)$. Slices were then washed again (TBS pH 7.5, $5 \mathrm{~min}$ ), blocked (1h, see FISH) and incubated with the anti-FITC-POD antibody (1:500, Roche, Ref.11426346910) for $2 \mathrm{~h}$ at $30^{\circ} \mathrm{C}$. Sections were then rinsed in washing buffer (TBS pH 7.5, 3x $10 \mathrm{~min}$ ) and TSACyanine 5 (1:50 in amplification diluent) (TSA Plus Cyanine 5 System, PerkinElmer Cat.NEL745001KT) was applied for $10 \mathrm{~min}$. Staining of nuclei and mounting was carried out as described above. 
Table 2. Primer sequence and probe length for in situ hybridation riboprobes.

\begin{tabular}{|c|c|c|c|c|}
\hline Gene & Forward primer & Reverse primer & $\begin{array}{l}\text { Probe } \\
\text { length } \\
\text { (bp) }\end{array}$ & Ref. \\
\hline$N d n f$ & gcgatgcacctttggagt & gcgatgcacctttggagt & 948 & (Boyle et al., 2011) \\
\hline Rgs8 & caggggagcattaagaaacaac & agaaagcaccagaaaacagagc & 767 & (Boyle et al., 2011) \\
\hline Rorb & acctgaagaccgagaccg & cccttcatttgcagaccg & 989 & (Boyle et al., 2011) \\
\hline Etv1 & gtgcctctgtctcactttgatg & ctactggcctgtgactcagttg & 801 & (Boyle et al., 2011) \\
\hline$T C_{1460681}$ & tcctcagagagtcagtccttcc & tactgtgatcggttttggagtg & 449 & (Boyle et al., 2011) \\
\hline $\operatorname{Trp73}$ & cctttgggttgactcctatcgac & cagaggaaggacaggagaagaa & 802 & $\begin{array}{c}\text { Based on } \\
\text { (Hanashima et al., } \\
\text { 2007) }\end{array}$ \\
\hline Ebf2 & ccgagatggattcggtcagg & cctcgtgtgtcagcagaac & 552 & $\begin{array}{c}\text { Own designed. } \\
\text { Based on } \\
\text { (Hanashima et al., } \\
\text { 2007) }\end{array}$ \\
\hline Pls3 & tcgggaagaaagaaccttcc & tccgattcttctagccatcg & 601 & $\begin{array}{c}\text { (Oeschger et al., } \\
\text { 2012) }\end{array}$ \\
\hline Gabra5 & tgactgctcacttccacctg & gagaggtggccccttttatc & 645 & $\begin{array}{l}\text { Oeschger et al., } \\
\text { 2012) }\end{array}$ \\
\hline Cdh10 & acgagcgactcaaagagcat & tcatttgcaagagcaagacg & 563 & $\begin{array}{c}\text { Oeschger et al., } \\
\text { 2012) }\end{array}$ \\
\hline Cdh18 & agccaaggtggaaaatgatg & ctctctgtccaggcttttgg & 615 & $\begin{array}{c}\text { Oeschger et al., } \\
\text { 2012) }\end{array}$ \\
\hline Unc5d & aacgtatgccctcacaggag & cgcagatcctctgtctgatg & 566 & $\begin{array}{c}\text { Oeschger et al., } \\
\text { 2012) }\end{array}$ \\
\hline Reln & tcagctggagaaaattagagcc & caagcactcagtgtggagtagg & 942 & Own desing \\
\hline
\end{tabular}

\section{Cell birth dating experiments}

Single pulse labeling. Adapted from: (Azim et al., 2009; Breunig et al., 2007; Vega and Peterson, 2005). Solutions of CldU (MW. 262.65, Sigma-Aldrich, Cat. C6891) or IdU (MW. 354.10, Sigma-Aldrich, Cat. 17125) were prepared at $5 \mathrm{mg} / \mathrm{ml}$ in sterile saline $(0.007 \mathrm{M} \mathrm{NaOH}, \mathrm{pH} 7.0)$ with strong shaking for $15 \mathrm{~min}$ (CldU) or $30 \mathrm{~min}$ (IdU). A single dose of the appropriate thymidine analog was administered by a single intraperitoneal (IP) injection at $10 \mathrm{ml} / \mathrm{kg}$ of animal weight to accomplish an effective dose of $57.5 \mathrm{mg} / \mathrm{kg}$ (IdU) or $43.5 \mathrm{mg} / \mathrm{kg}$ (CldU). A $10 \mathrm{mM}$ solution of EdU in DMSO was prepared following the manufacturer's instructions (Click-IT Plus EdU Alexa Fluor 647 Imaging Kit, ThermoFisher cat.C1064) and a single-dose IP-injected at $3.3 \mathrm{ml} / \mathrm{kg}$ of animal 
weight. EdU was administered at E13.5, CldU at E14.5 and IdU at E15.5. Embryos were dissected 2 days (E17.5), 4 days (PO) and 11 days (P7) after the injection, and brains were sectioned at 14 $\mu \mathrm{m}$ and processed for immunostaining.

Continuous BrdU labeling. Osmotic pumps (Alzet, \#1003D) were filled with a $16 \mathrm{mg} / \mathrm{ml}$ BrdU (MW. 307.10, Sigma-Aldrich, Cat. B5002) solution (in 1:1 DMSO:water) as described by the manufacturer. Pumps were incubated overnight at $37^{\circ} \mathrm{C}$ to allow initial priming. The pump was then placed in the peritoneal cavity of the pregnant mouse following the FlashTag (FT) intraventricular injection (at E13.5 or E15.5), for a 3-day chronic BrdU administration. Embryos were collected 4 days after the FT injection and brains were processed for tissue analysis as described before.

\section{In utero electroporation}

In utero electroporation of the cerebral cortex was performed as described in (Saito, 2006; Saito and Nakatsuji, 2001). Pregnant mice from C57BL/6 or IR/CD-1 strains were pre-anesthetized by placing the mice in an isoflurane-containing chamber ( $3 \%$ isoflurane flow, $1 \mathrm{~L} / \mathrm{min} \mathrm{O}_{2}$ ). Once anaesthetized, the animals were removed from the chamber and placed on their backs on a heating pad (set to $37^{\circ} \mathrm{C}$ ). General anesthesia was maintained via mask (1-2\% isoflurane flow and $0.8 \mathrm{~L} / \mathrm{min} \mathrm{O}_{2}$ ). Eye drops (Bepanthen, Bayer) were used to provide moisture and prevent corneal ulceration. Carprophen or buprenofine (i.m. injection) was administer for analgesia. Absence of foot and tail reflexes were checked before starting the surgery.

After cleansing the skin with an antiseptic (Kodan, Shülke), a small vertical incision was made along the midline through the abdominal skin, the linea alba and the peritoneum. The animal was then covered with a sterile gauze. Gauze and abdominal cavity were kept moist with warm sterile $0.9 \% \mathrm{NaCl}$. The uterine horns were then gently pulled out and placed on the wet gauze. For the time of the surgery, the uterine horns were always kept moist and warm with sterile $0.9 \% \mathrm{NaCl}$. Pre-pulled micropipettes were used for injection, using a pedal-controlled microinjector. One $\mu \mathrm{l}$ of the DNA solution were injected into the lateral ventricle of each embryo. The endotoxin-free plasmid DNA $(2.5 \mu \mathrm{g} / \mu \mathrm{l})$ was diluted in $10 \%$ Fast Green $(2: 1)$, to allow the visualization of the injection. For the electroporation, 5 pulses of $50 \mathrm{~ms}$ length, separated by 900 ms pause, were delivered at $33 \mathrm{mV}$ for E14.5 embryos and at 35V for E15.5 embryos (Nepa21 electroporator, NepaGene 2720114). To ensure efficient current conduction, $(5 \mathrm{~mm})$ paddle electrodes 
(NepaGene, C115CB-2) and uterus were kept moist with saline. Typically, three to four embryos per pregnant dam were electroporated in IR/CD-1 strain, while all embryos were injected and electroporated in C57BL/6 dam (ratio of 1:10 reeler mice per litter). After the electroporation, embryos were returned into the peritoneal cavity and warm sterile $0.9 \% \mathrm{NaCl}$ was poured in the abdominal cavity. The abdominal wall was then sutured (Ethicon, 6951H) and the skin was closed with a 9 mm stapler (FST, 12031-09; Reflex9, 201.1000). Lidocaine (4\%) was applied to the wound. Additionally, a day before and two days after the surgery, Carprofen $(50 \mathrm{mg} / \mathrm{ml})$ diluted 1:100 in water was available ad libitum. The animal was left to recover from the anesthesia on the heating pad for 2 hours and transferred to a new clean cage (place on a heating pad). Embryos were left in utero and were collected after 2 days. Brains were dissected out and screened for GFP expression, before fixation, using a fluorescence stereomicroscope (Nikon, C-DSA230). Tissue was fixated and sectioned as described above.

Preparation of micropipettes for DNA injection. Glass capillaries for injection were pulled from borosilicate glass (GB150F-8P, Science Products) using a P-97 puller (Sutter Instruments), under the following conditions: pressure, 50; heat, 800; pull, 30; velocity, 40; time, 1 .

\section{Plasmids}

All plasmids used for in utero electroporations contained GFP as a reporter gene downstream of the CAG promoter (cytomegalovirus immediate early enhancer, chicken beta-actin promoter and rabbit beta-globin (Niwa et al., 1991)). The CAG promoter provides a strong expression that drops rapidly in the $\mathrm{VZ}$ after the electroporation (giving a birth date-specific expression) and persist in differentiated neurons after birth. The constructs used for the experiments were: pCAG-EGFP (original source: Dr. Noriko Osumi, kindly provided by Dr. Tran Tuoc), pCIG2 (also known as pCAGIRES-EGFP, original source Francois Guillemot's Laboratory, kindly provided by Dr. Tran Tuoc), pCAG- ApoER2 $2^{\text {Dyk }}$-IRES-EGFP and pCAG-VIdlr ${ }^{\text {Dyk }}$-IRES-EGFP. DNA plasmids were purified using the EndoFree Plasmid Kit (Quiagen, Cat. 12362) according to the manufacturer's instructions.

Overexpression plasmids. ApoER2 (also known as Lrp8) and VIdlr pcDNA3.1+- ${ }^{\text {DYK }}$ encoding vectors were purchased from GeneScript (Clone IDs: OMu23075 and OMu22960, respectively). In these plasmids the expression of the cDNA is driven by the cytomegalovirus (CMV), which expression is generally low, does not persist after neuronal maturation and does not harbor any reporter gene. Consequently, the sequences (including the DYK N-terminal tag) encoding the 
transcript variant(s) 2 for ApoER2 and VIdlr were subcloned into the pCIG2 vector by blunt-end cloning, to generate pCAG-ApoER2 ${ }^{\text {Dyk}}$-IRES-EGFP and pCAG-VIdlr ${ }^{\text {Dyk }}$-IRES-EGFP.

In summary, $1 \mu \mathrm{g}$ of the ApoER2 or the VIdlr pcDNA3.1-DYK vector (donor plasmids) was restriction digested. Ends were filled using the DNA Polymerase I large, known as Klenow fragment (Roche, \#11 008404001 ), and dNTPs (Thermo Scientific, R0181) (incubation at $37^{\circ} \mathrm{C}$ for $45 \mathrm{~min}$ ). In parallel, $1 \mu \mathrm{g}$ of $\mathrm{pClG} 2$ (recipient plasmid) was restriction digested by Smal (ThermoFisher, ER0661), producing a linearized blunt-ended plasmid, and dephosphorylated by the Antarctic Phosphatase (New England Biolabs, M0289) (15 min at $37^{\circ} \mathrm{C}$ incubation time), to avoid self-ligation. Reaction products were then loaded in a 1.5\% agarose gel, and inserts and linearized recipient vector were isolated by gel purification (NucleoSpin Gel and PCR Clean-up, Macherey-Nagel). Subsequently, insert(s) and plasmid were ligated, by using a T4 DNA ligase plus a PEG-rich ligation mixture (ThermoFisher, EL0011), with the following specifications: molar ratio of vector to insert 1:3 or 1:5, at least three ligations for each plasmid and with negative controls in parallel.

Once ligation was accomplished, $\mathrm{DH} 5 \alpha$ E.coli-competent bacteria were transformed and plated as described above. Plasmid preparation was carried out as described before. Plasmids were afterwards digested with the appropriate enzymes, loaded in a 1.5\% agarose gel and sent for sequencing. After persevering, LB-supplemented medium was inoculated with the validated single-colony cultures, amplified overnight (at $37^{\circ} \mathrm{C}$ at $150 \mathrm{rpm}$ ) and purified by using the EndoFree Plasmid Kit (Quiagen, Cat. 12362).

\section{Transfection, Immunoblotting and Immunocytochemistry}

Hek293 o Neuro2A cells were cultured in Dulbecco's modified Eagle's medium (Sigma, D5796) supplemented with $0.05 \%$ streptomycin- penicillin (Life Technologies, $1570-063$ ) and 10\% fetal bovine serum (FBS; Gibco, $160000-044$ ) at $37^{\circ} \mathrm{C}$ and $5 \% \mathrm{CO}_{2}$ atmosphere, and handled following the standard protocols for cell culture. Prior transfection, cells were seeded onto multiwell plates (with glass coverslips for the cells intended to be immunostained) and grown in culture medium. 90\% confluent cells were then transfected with pCAG-IRES-EGFP, pcDNA3.1-ApoER2 ${ }^{\text {Dyk, }}$

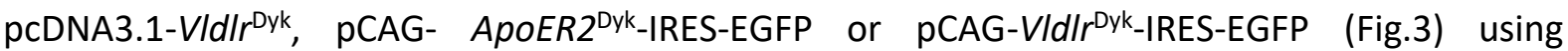
Lipofectamine 2000 (Invitrogen, Cat.11668-019) in accordance with the manufacturer's 
instructions. Two days post-transfection, cells were processed for immunoblotting or immunocytochemistry.

Immunoblotting. Preparation of lysates from transfected cells were done as follows: transfected adherent cells were trypsinized (TryLE; Life Technologies, 12605010) and pellets were resuspended in 2X SB buffer (non-reducing sample buffer; Thermo Scientific, 39001). Samples were then sonicated (Bandelin Electronic, GM2070) for one cycle and protein denaturation was facilitated by adding 4\% 2-mercaptoethanol. Protein samples were then separated on $10 \%$ sodium dodecyl sulfate (SDS) polyacrylamide gels (SDS-PAGE, 80-120 V for $1.5 \mathrm{~h}$ ) and transferred to a polyvinylidene fluoride (PDVF) membrane via electrophoresis (100 V for $90 \mathrm{~min}$ ). Blots were washed (PBS, $10 \mathrm{~min}$ ), blocked in Tris-buffered saline (TBS) $0.2 \%$ with $5 \%$ milk powder for $1 \mathrm{~h}$, and incubated overnight at $4^{\circ} \mathrm{C}$ in: mouse anti-GAPDH IgG1 (1:2000, OriGene, TA802519), rabbit antiVIdlr (1:1000; Abcam, ab92943), rabbit anti-ApoER2 (1:10.000 and 1:50.000; Abcam, ab108208) or mouse anti-DYDDDD Tag IgG2b (1:500; GeneScript, Cat.A00187-200) antibodies diluted in blocking solution. After rinsing (TBS $0.2 \%, 3 \times 15 \mathrm{~min}$ ), membranes were incubated with the appropriate horseradish peroxidase (HRP)-conjugated secondary antibodies (1:2000 - mouse and rabbit IgG HRP; Dianova, 115035003) in blocking solution for 2 hours. Blots were then washed (TBS $0.2 \%, 3 \times 15 \mathrm{~min}$ ), incubated for $1 \mathrm{~min}$ in the chemiluminescent reagent, prepared according the to the manufacturer's instructions (SuperSignal ${ }^{\mathrm{TM}}$ West Pico Chemiluminescent Substrate; ThermoFisher, Cat.34079), and exposed to films (Kodak X-OMAT F5388) for band detection. For reprobing, membranes were incubated in $1 \mathrm{x}$ antibody striping solution for $15 \mathrm{~min}$ ( $\mathrm{Re}$ BlotWestern Blot Recycling kit, Chemicon, Cat. 2060), washed and blocked before the incubation with the primary antibodies.

Immunocytochemistry. Two days post-transfection, cells were washed (PBS, 3x $10 \mathrm{~min}$ ) and fixated in 4\% PFA for 30 minutes. Cells were rinsed (PBS, $2 \times 5 \mathrm{~min})$, blocked $\left(50 \mathrm{mM} \mathrm{NH}_{4} \mathrm{Cl}, 10\right.$ $\mathrm{min}$ ), washed (PBS, $2 \times 5 \mathrm{~min}$ ), permeabilized (PBS 0.2\% Triton X-100, 3x $4 \mathrm{~min}$ ) and incubated for 1h with mouse anti-DykDDDDTag IgG2b antibody (1:500; GeneScript, Cat.A00187-200) diluted in permeabilization buffer. Following rinsing (PBS 0.2\% Triton X-100, 3x 4min), cells were incubated in the secondary antibody (1:500, anti-mouse AlexaFluor 568 IgG2b; Invitrogen, Cat. A21144) diluted in permeabilization buffer for $1 \mathrm{~h}$. Samples were washed several times (PBS $0.2 \%$ Triton X100, 3x 4 min; and PBS, 2x 5 min), nuclei were counterstained with DAPI and coverslips mounted on a slide glass in Aqua-Poly/Mount. 
A

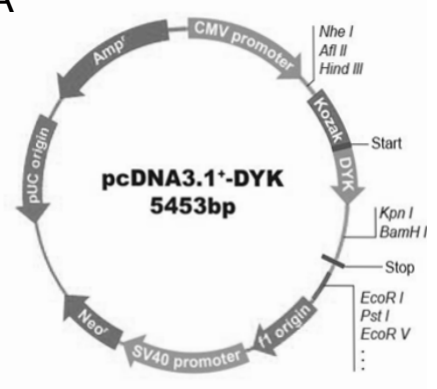

B

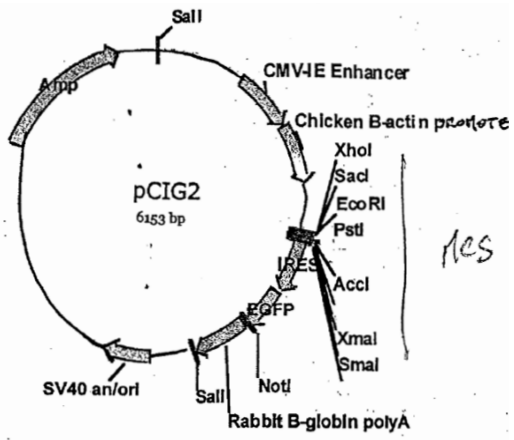

C

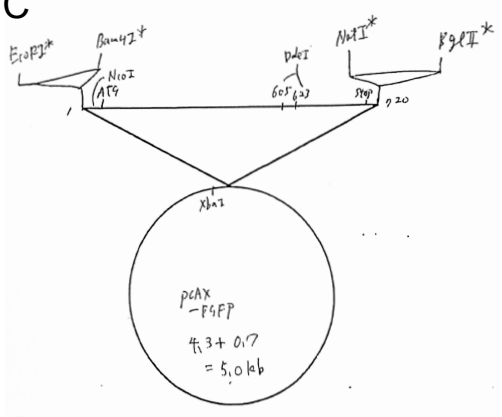

Figure 3. Vector maps. Expression vectors used for immunocytochemistry and/or in utero electroporation experiments $(A, B, C)$. Coding sequence for ApoER ${ }^{\text {Dyk }}$ and VIdll ${ }^{\text {Dyk }}$ was inserted in $\mathrm{PClG} 2$ by blunt-end cloning. pCIG2 and pCAX express eGFP under the cytomegalovirus immediate early enhancer and chicken â actin promoter fusion (CAG) promoter.

\section{Flash Tag}

Flash Tag injections were performed as described in (Telley et al., 2016) with slight adjustments. Carboxyfluorescein succinimidyl ester (CellTrace ${ }^{\mathrm{TM}}$ CSFE, Invitrogen Cat. C34554) was prepared at $5 \mathrm{mM}$ in DMSO, prior to use. Timed-pregnant females were prepared for surgery as described for in utero electroporation and $0.5 \mu \mathrm{l}$ of CFSE (diluted 1:2 in 1X Fast Green) was injected into the ventricles of E13.5 or E15.5 embryos. Brains were dissected out 2, 4 or 11 days after the FT injection. If required, a single i.p. injection of $\mathrm{EdU}(10 \mathrm{mM}, 3.3 \mathrm{ml} / \mathrm{kg}$ of animal weight) was administered into the intraperitoneal cavity before suturing.

\section{Image Acquisition}

Brightfield images were acquired with an Axio ImagerM2 (Zeiss) controlled by the Neurolucida software (MBF Bioscience, Version11). Images were recorded as image stacks (7 z-planes $5-\mu m$ steps for Figs. 5, 6, 7, 10; 7 z-planes 1- $\mu$ m step for Fig. 11) and are shown as minimum intensity projections. To merge the laminar marker images (Fig.1), an individual false color was assigned to every micrograph with an individual laminar maker. The consecutive series of the 5 laminar 
markers was merged into one image using Photoshop CS6 (Adobe). Fluorescent images were acquired: a) using the same microscope, but with a structured illumination module (ApoTome, Zeiss) controlled by the Neurolucida software, or b) using a spectral confocal microscope (TCS SP5, Leica). Confocal micrographs were acquired as image stacks (40x, $10 \mathrm{z}$ planes with 2- $\mu \mathrm{m}$ steps; 63x, $10 \mathrm{z}$ planes with 1- $\mathrm{mm}$ steps) and are presented as maximum intensity projections. Appropriate image corrections (e.g. optimized tonal range and color balance levels) were done in Adobe Photoshop CS6 or FIJI. Figures were arranged in Adobe InDesign CS6. Adobe Illustrator CS6 or CorelDraw X6 were used to modify (Figure 1) or create de-novo the schematics (Figures 2, 19, $22,26,27,33$ and 34).

\section{Quantifications and Statistical Analysis}

Animals, from a minimum of two separate litters (if applicable), included in each set of experiments were as follows:

- $\quad n=12$ adult animals in the laminar maker project. $n=4-6$ for each sensory area and per phenotype (200 sections in total)

- $\quad n=14$ animals for the analysis of preplate splitting, being: $n=3$ for probe validation, $n=3-4$ for dFISH experiments, $\mathrm{n}=2-3$ for Calr immunostaing in reeler, and $\mathrm{n}=4$ for tdTomato expression in ROR $\alpha^{\mathrm{tdTom}}$ animals (per area and genotype).

- $\quad n=26$ animals for the halogenated thymidine analogs experiments, being analyzed $n=3-6$ animals per sensory area, genotype and developmental time point.

- $n=22$ for FlashTag- injected animals, $n=2-4$ analyzed per area, genotype and developmental time point.

- $\quad n=2-3$ independent experiments for the immunocytochemistry and immunoblotting assays.

- $\quad n=8-10$ for in utero electroporation assays, being considered at least $n=2$ for plasmid.

Data were processed in Excel (Microsoft) and statistical analysis was done in Sigma Plot 12 (Systat). Significance was tested using two-tailed t-tests, Mann-Whitney rank-sum tests or twoway ANOVA (multiple comparisons). The level of significance was set to ${ }^{*} \mathrm{P}<0.05 ;{ }^{*} \mathrm{P}<0.01$; ${ }^{* * *} \mathrm{P}<0.001$. Bar graphs are plotted as mean (SD) or standard error of the mean (sem). If possible, littermates where use for comparison. 
Lamina- fate markers. Neurons expressing the particular laminar marker were registered manually (200 sections), directly from the slide, using Neurolucida (MBF Bioscience, Version11) and the brightfield microscope (Zeiss Imager-M2). Prior to the experiment, coronal sections, containing the corresponding area of interest, were chosen based on a mouse brain atlas (Paxinos and Franklin, 2004): FrA, Bregma 2.96 - 2.58 mm; M1, Bregma $1.34-0.62$ mm; S1BF, Bregma $1.22--2.06 \mathrm{~mm}, \mathrm{~V} 1$ and Au1, Bregma $-2.54--3.16 \mathrm{~mm}$. Columns (600 $\mu \mathrm{m}$ width, $40 \mu \mathrm{m}$ depth) were delineated manually based on anatomical references from the same atlas. Relative distance of the individual cells to the pia mater was exported to Excel with NeuroExplorer (MBF Bioscience). For the analysis, columns were divided it into 10 or 20 bins of equal size normalized to the height of the column. The density of neurons was normalized to cells per $\mathrm{mm}^{3}$.

Neuronal orientation. The nuclear counterstaining (DAPI) was used in the in utero electroporation experiments to discriminate $V Z, I Z$ and $C P$, and only cells that resided in the IZ were taken into account for the cell orientation assessment. The potential direction of the electroporated cells was analyzed based on the Golgi orientation (position of GM130 immunofluorescence relative to a cell soma) and, if detectable, the direction of the leading process. Two major quadrants (Q1 and Q2, 180 degrees each) were assigned to a cell soma and cell direction was categorized as either VZ- or CP-oriented (Q1 and Q2, respectively). Quantifications were done manually in (n) z-stack images using Neurolucida and data were exported and processed as described above.

(Cell) birth dating analysis. Prior to the experiment, coronal sections, containing the corresponding area of interest, were chosen based on developing mouse brain atlas (applicable for all studies at developmental stages) (Paxinos et al., 2011). Thymidine analogs and FlashTag fluorescence micrographs were analyzed by a custom-written script in ImageJ (v1.51r, NIH), results exported to RStudio (v.3.4.2, CRAN.R-project) and further analyzed using an additional custom-written script. Scripts were written by Pavel Truschow. In summary, raw images acquired as image stacks (Axiolmager.M2, Zeiss or TCS SP5, Leica) were imported as image sequences (individual channels, planes and tiles separately), sequences were merged individually and tiles were stitched by pairwise stitching in ImageJ. The essence of the script for the automated analysis in ImageJ is as follows: analysis was carried out in non-processed images with a minimum of $5 \mu \mathrm{m}$ thickness (6 z-stacks), background subtraction was estimated based on the mean intensity value of each image and cells were detected as regions of interest (ROIs) based on the following parameters: adaptive threshold: $75,-10$; outliers exclusion: 3.0 radius, 50 bright; particle analysis: 
minimum cell size $15 \mu \mathrm{m}$, circularity: 0.5-1.00. Quantification of double and triple-labeled cells was based on the comparison of sum projection masks for each individual channel. Total number, $x$ - and $y$-coordinates and intensity values for each ROI were exported as csv.files. Thresholding (50\% highest fluorescence intensity in the thymidine analogs labeled sections, and $50 \%, 30 \%$ and $20 \%$ highest fluorescence intensity for the FlashTag experiments), data normalization according to the height of the micrograph (based on DAPI cells coordinates) and plotting (as violin plots) was accomplished using RStudio. Further data analysis and plotting (bar and line charts) was done in Excel and SigmaPlot. If applicable, the cortical column was divided into 20 bins of equal size, as described above. 


\section{RESULTS}

\section{THE MANY FACES OF THE REELER CORTEX}

The reeler neocortex is often described as a model where projection neurons are positioned abnormally in an outside-in or inverted pattern (Caviness, 1982b; Caviness and Sidman, 1973; Cooper, 2008; Rice and Curran, 2001; Sekine et al., 2012), with just a few alternative notions being found in the literature (Boyle et al., 2011; Dekimoto et al., 2010; Ferrere et al., 2006; Polleux et al., 1998; Wagener et al., 2010). The abnormal architecture of the neocortex happens to emerge due to a lack of Reelin expression, a protein that is considered the master of ceremonies of neuronal migration and cell positioning (D'Arcangelo, 2014). However, in a structure that starts with a few thousand immature cells and ends with billions of adult neurons, with a highly complex temporal and spatial signaling pathways that regulate brain development, the idea of reeler and its inverted cortical lamination should be considered an oversimplification (Boyle et al., 2011; Dekimoto et al., 2010). Thus, by using up-to-date methods, the area-specific effects and the complexity of the Reelin-deficiency can be reevaluated.

The lamination of the adult cerebral cortex was assessed in wild type and reeler mice by taking advantage of lamina (or layer)- fate markers. These are genes whose expression is restricted to certain laminae. The expression of $N d n f, R g s 8, R o r b, E t v 1$, and $T C_{1460681}$ was detected by chromogenic in situ hybridization (ISH) in coronal sections of adult wild type and reeler cortex. Probe generation and ISH were performed as previously described (Wagener et al., 2016). The results are shown as: a) pseudo-colored overlays of (five) consecutive sections (Fig.4), to facilitate the comparison of the laminated patterns between the two genotypes, or b) as bright field micrographs of individual markers (overviews in Fig.5 and 6, and insets in Fig.7 and Fig.10), to evaluate each layer separately. Cortical lamination was examined in detail in the frontal association area (FrA; Fig.4 A-B; Fig.5 A-B and Fig.7 A), primary motor cortex (M1; Fig.4 C-D; Fig.5 C-D and Fig.7 B), primary somatosensory 'barrel' cortex (S1BF; Fig.4 E-F; Fig.6 A-B and Fig.7 C), primary auditory cortex (Au1; Fig.4 G-H; Fig.6 C-D and Fig.7 D) and primary visual cortex (V1; Fig.4I-J; Fig.6 C-D and Fig.7 E). Line charts (based on manual cell counting, $n=4-6$ animals per genotype per lamina-fate marker; Fig.4, Fig.8 and Fig.10) show the spatial distribution of the distinct laminafated cells over the cortical wall in the given area. To facilitate the analysis, the cortex was divided 
into 20 bins of equal size (normalized to the dimensions of each cortex), where the lowest bin number is, the closest to the surface of the brain.

The lamina- fate markers showed a strong and unique regional enrichment, allowing the delineation of cortical layers in all analyzed areas of wild type animals (Fig.4 A, C, E , G ,I and primes). $T C_{1460681}$ (shown in blue) defines a subpopulation of cells in the polymorphic layer VI, Etv1 (shown in pink) outlines layer $\mathrm{Va}$ (together with layer $\mathrm{Vb}$ and layer $\mathrm{VI}$ define the infragranular layers), Rorb staining (shown in red) is enriched in the granular layer (layer IV), Rgs8 (shown in orange) labels cells in layer II/III (supragranular layers) and Ndnf (shown in yellow) stains cells located immediately below the pia mater, corresponding to layer I (known as the molecular layer). As described before, sensory areas, such as the primary visual cortex and the primary somatosensory cortex, have a robust layer IV, which is a main receiver of sensory input (Kandel et al., 2013). Cortical areas involved in the control of movement are classically described as agranular (Garey, 1999; Kandel et al., 2013). However, here we show a thin but detectable Rorb layer in the primary motor cortex. It thus indicates that the motor cortex has a layer IV, as others pointed out previously (Gatter et al., 1978; Meyer, 1987; Skoglund et al., 1997; Yamawaki et al., 2014). Together, our set of probes revealed the well- ordered lamination of the wild type cortex, where early-born neurons (layer VI and layer V cells) are restricted to lower layers and late-born neurons (layer IV and layer II/III cells) are located superficially.

In order to address the doubts raised in the literature about the seemingly inverted cortex in reeler, the expression pattern of the same layer-fate markers was analyzed and quantified in the same cortical areas of Reelin-deficient mice. The data demonstrate that the cytoarchitectural organization of the reeler cortex, although highly disrupted, is undoubtedly not simply inverted. Instead, the neocortex of the mutant mouse shows major and unique areal differences across the anterior-posterior axis (Fig.4 B, D, F, H, J and primes). To begin with, the FrA shows a broad cell dispersion, with Rgs8- neurons scattered from side to side of the cortical depth, and Rorb and $T C_{1460681}$ expression restricted to upper cortical levels. Overall, the (broad) distribution of layer II/III (Rgs8)- neurons is followed by layer IV (Rorb)- and layer V (Etv1)- fated cells (Fig.4 B', Fig.5 B, Fig.7 A). In M1, layer II/III, IV and V fated cells are massively intermingled, but remained approximately in their correct relative (quasi- wild type) order. Thus, Rgs8- neurons are largely positioned in the upper cortex, Rorb expression is restricted to the mid-cortical level and the sparse Evt1 expression is inclined to lower layers. These cells are sandwiched by layer VI fated cells (Fig.4 D', Fig.5 D, Fig.7 B). The lamination pattern of S1BF differs from those located more 
rostrally. In this scenario, layer II/III and layer IV fated cells are sandwiched by layer V and VI fated cells. Strikingly, regardless of the layer disappearance, Rorb- neurons (layer IV) still aggregate, resembling barrel-like structures (Fig.6 B, empty arrowheads) (Fig.4 F', Fig.6 B, Fig.7 C). Conversely, Au1 shows a mirror-image pattern, with layer IV and layer V neurons located in midcortical levels flanked by superficial and deep bands of layer II/III neurons (Fig.4 H', Fig.6 D, Fig.7 D). However, in V1, despite massive cellular intermingling, the distribution of the main laminar compartments indicate at least a tendency of an inversion. Thus, in the most caudally located area examined, the earlier generated cells ( $T C_{1460681^{-}}$and Etv1- expressing cells) are settled in a superficial location while late-born neurons (Rgs8- neurons) are found below (Fig.4 J', Fig.6 D, Fig.7 E).

Figure 4. Disturbed cortical layering of the Reelin-deficient neocortex in an area-specific fashion. Expression of $N d n f$ (LI; yellow), Rgs8 (LII/III; orange), Rorb (LIV; red), Etv1 (LV; purple) and TC1460681 (LVI; blue) were detected by chromogenic in situ hybridizations on rostral (A-D), medial (E-F) and caudal (G-J) serial sections of adult wild type (wt) and reeler ( $\mathrm{rl} / \mathrm{/}-\mathrm{)}$ mice, shown as pseudo-colored mergers. Density plots represent the spatial distribution of the different neuronal populations in the frontal association area (FrA), primary motor cortex (M1), primary somatosensory 'barrel' cortex (S1BF), primary auditory (Au1) cortex and primary visual cortex (V1). Notice that the wild type cortex reproduces the well-known (horizontal) layering of the cortex (Roman numerals), whereas the reeler cortex displays different patterns of horizontal cytoarchitectural disorganization over the rostral to caudal axis. Abbreviations: wm, white matter. Scale bars: $1000 \mu \mathrm{m}(A-J), 200 \mu \mathrm{m}\left(A^{\prime}-J^{\prime}\right)$.

Figure 5. Marker-based phenotyping in the frontal association area and the primary motor cortex. Chromogenic in situ hybridizations for Ndnf (LI), Rgs8 (LII/III), Rorb (LIV), Etv1 (LV) and TC 1460681 (LVI) on serial coronal sections that host the FrA and the M1 (arrowheads) of adult wt $(A, C)$ and $r l-/-(B, C)$ mice. Scale bars: $1000 \mu \mathrm{m}$.

Figure 6. Marker-based phenotyping in sensory areas: primary somatosensory cortex, primary visual cortex and primary auditory cortex. Chromogenic in situ hybridizations for Ndnf (LI), Rgs8 (LII/III), Rorb (LIV), Etv1 (LV) and $T C_{1460681}$ (LVI) on serial coronal sections that host the S1BF (A, B filled arrowheads), V1 (C,D filled arrowheads) and Au1 (C,D empty arrowheads) of adult wt (A,C) and $r$ l/- $\quad(B, C)$ mice. Despite the absence of layers in reeler, Rorb expressing cells (LIV) still aggregate resembling barrel-equivalent structures (B, empty arrowheads). Scale bars: $1000 \mu \mathrm{m}$.

Figure 7. Differential (mis)lamination in cortical areas in the adult reeler neocortex. Expression of $N d n f(L I)$, Rgs8 (LII/III), Rorb (LIV), Etv1 (LV) and TC 1460681 (LVI) were detected by chromogenic in situ hybridizations in the FrA (A), M1 (B), S1BF (C), Au1 (D) and V1 (E), on serial sections of adult wt and $r l-/$ - mice. Wild type exhibits a strict laminar segregation of Rgs8, Rorb, Etv1, $T C_{1460681}$ expressing neurons through the anteroposterior axis of the cortex. The reeler cortex, although greatly disrupted, is undeniably not simply inverted. Instead, the neocortex of the mutant mouse shows major and unique areal differences, which roughly range from a wild type-equivalent order in M1 (B) to a quasi-inverted pattern in V1 (E). Scale bars: $100 \mu \mathrm{m}$. 

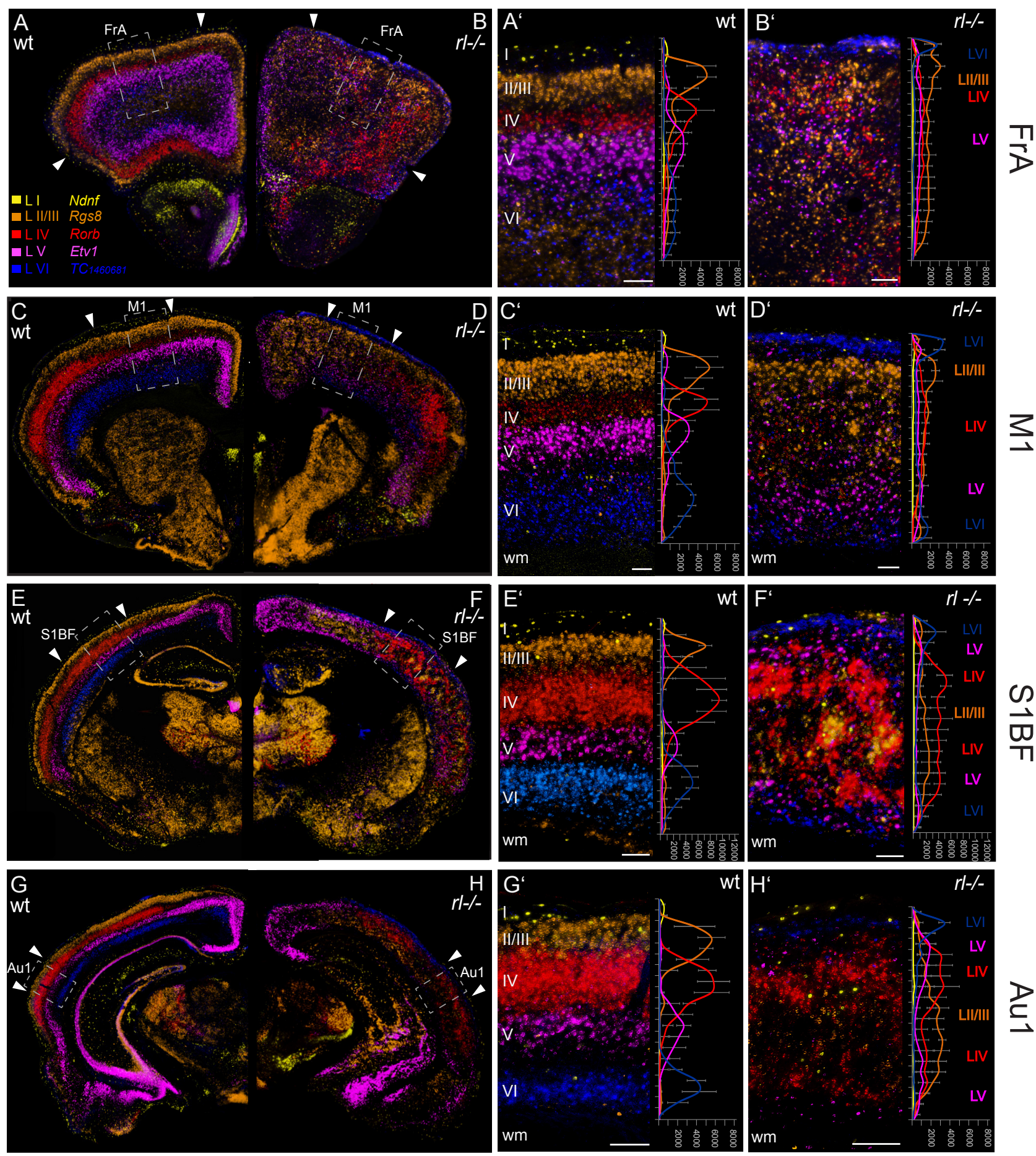

$\underset{\longrightarrow}{Z}$
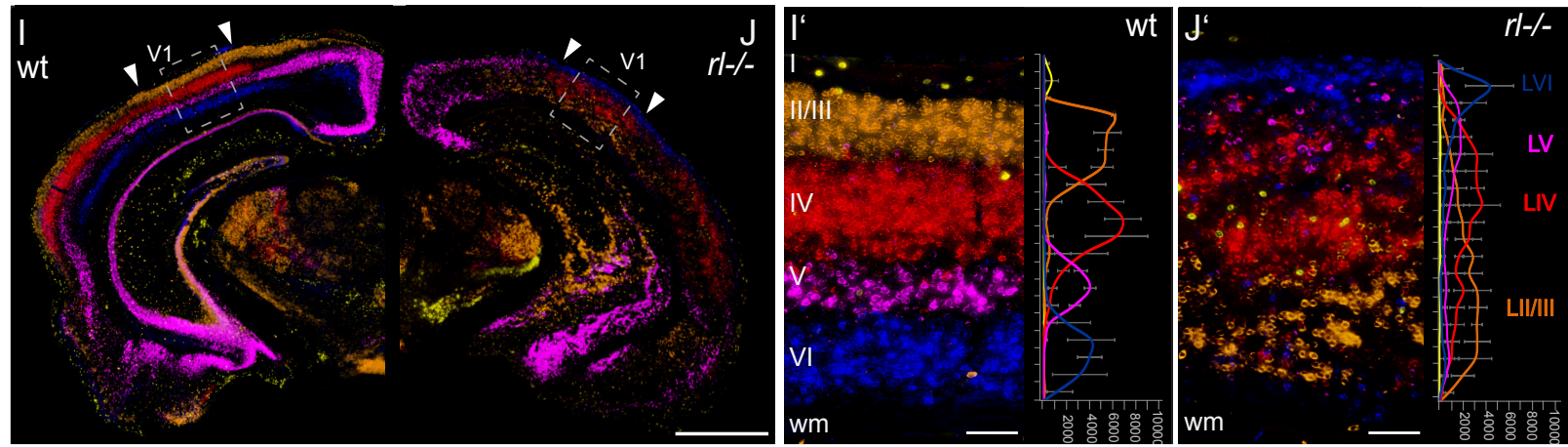

$\leq$

Figure 4. Disturbed cortical layering of the Reelin-deficient neocortex in an area-specific fashion. 
FrA

M1

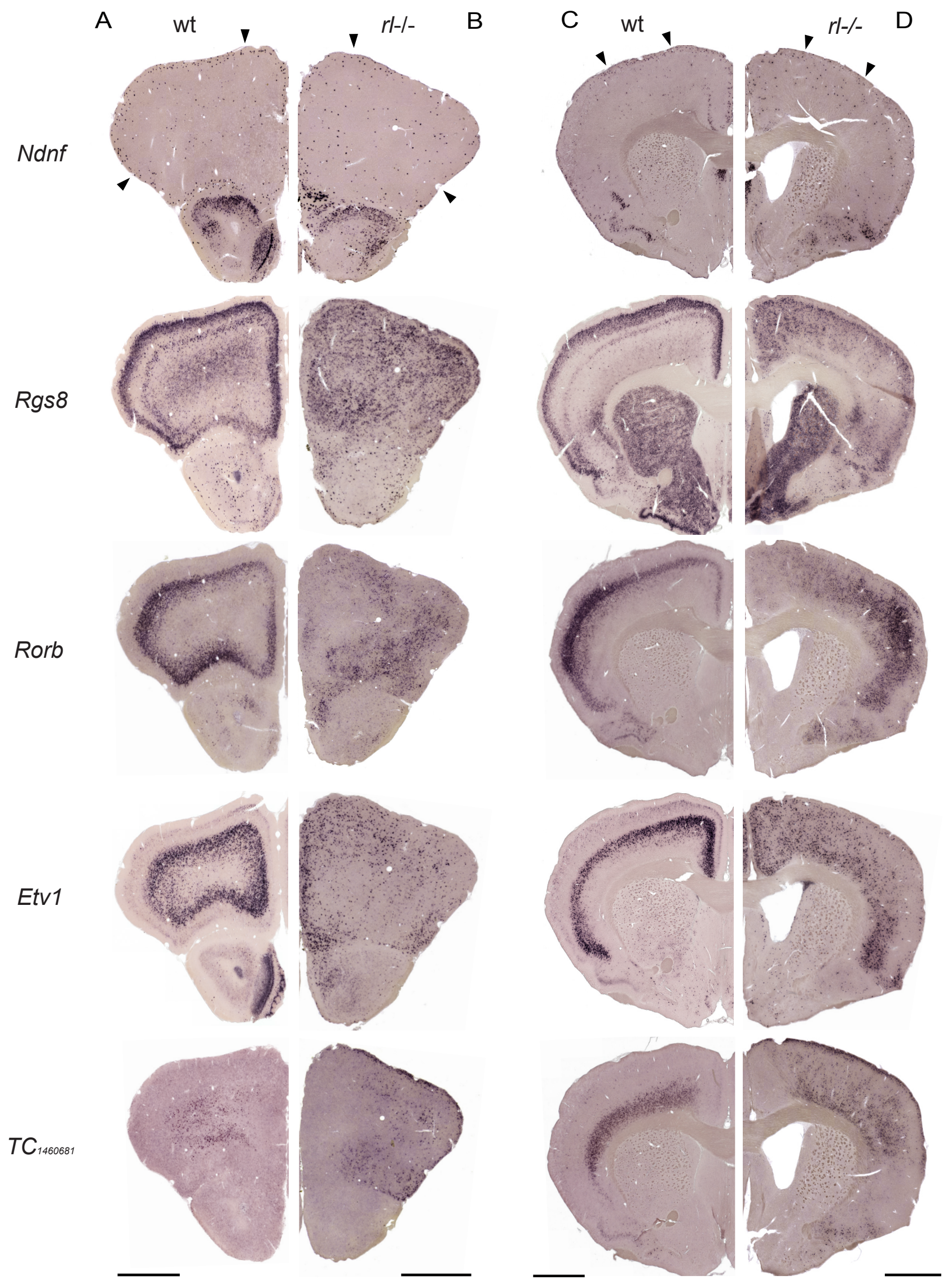

Figure 5. Marker-based phenotyping in the frontal association area and the primary motor cortex. 


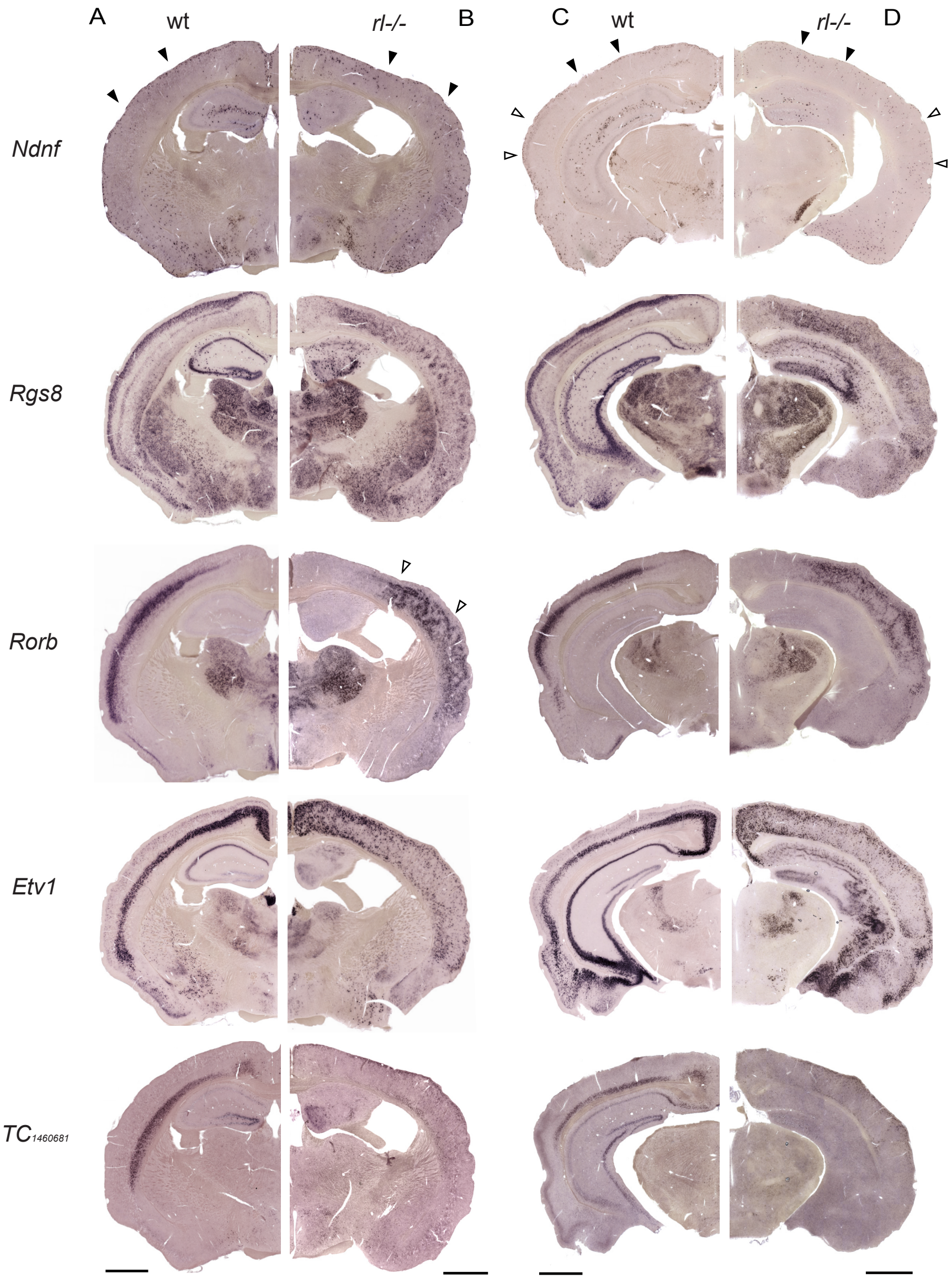

Figure 6. Marker-based phenotyping in sensory areas: primary somatosensory cortex, primary auditory cortex and visual auditory cortex. 

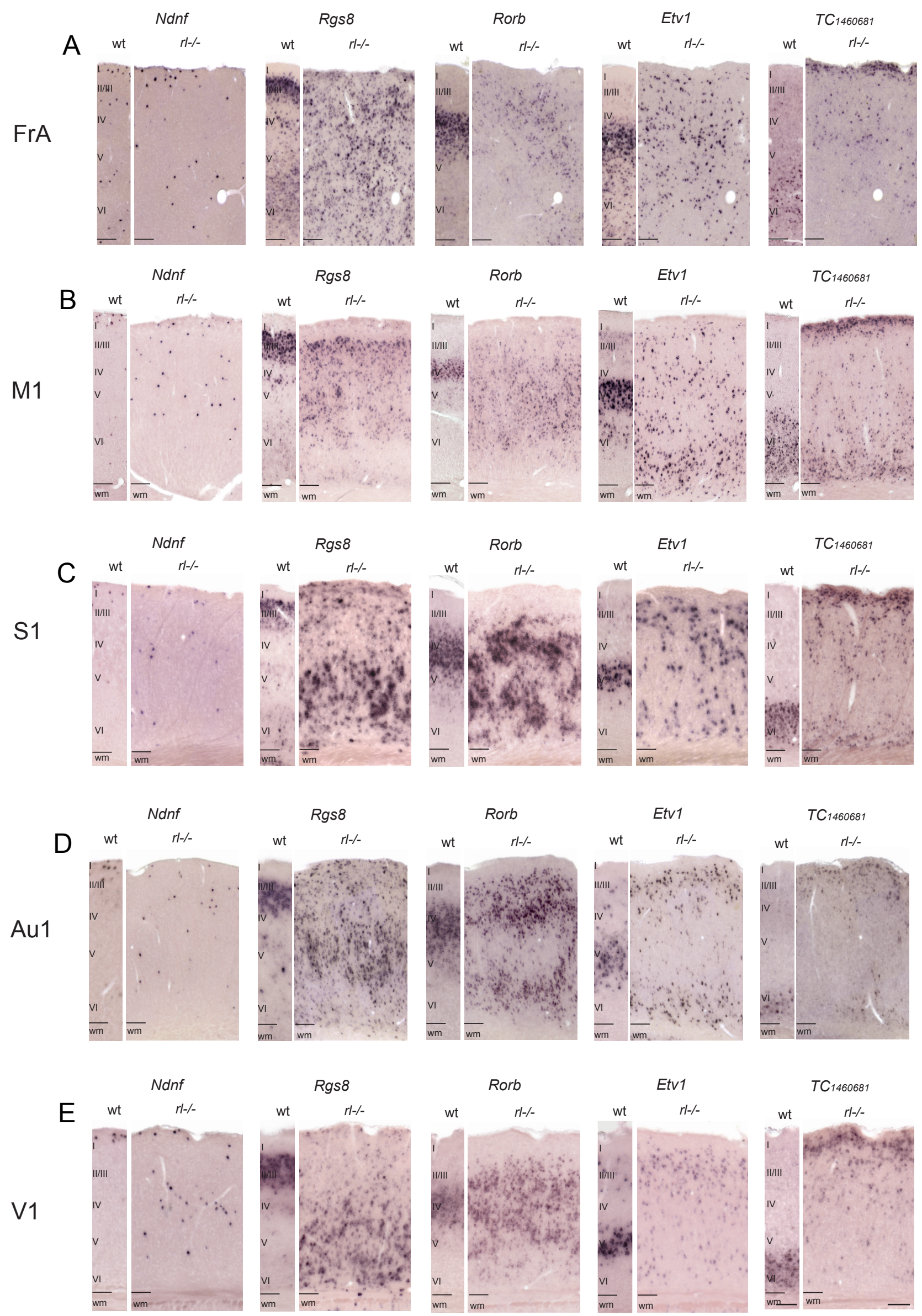

Figure 7. Differential (mis)lamination in cortical areas in the adult reeler neocortex. 
Notice that, even though cells are not organized into well-defined layers in the Reelindeficient neocortex, the location of cell bodies expressing the corresponding layer- marker is not random, as cell density peaks at approximately the same bin range for a given layer- marker in a certain cortical area (Fig.8). This circumstance is remarkably constant for all reeler animals ( $\mathrm{n}=4$ 6) and cortical areas included in the study, indicating therefore, that despite an apparent neocortical lamination chaos, there seems to be some sort of control in the positioning of projection neurons.

Besides, and in agreement with previous studies (Caviness and Sidman, 1973), neither cortical thickness nor cell density were significantly different when comparing the two genotypes (Fig.9), with minor exceptions (cell density: FrA Rgs8 - Two Way ANOVA, $p=0.0371$; cell density: S1BF Rorb - Holm-Sidak method, $\mathrm{p}=0.014$; cortical thickness S1BF - Mann Whitney Rank Sum Test, $\mathrm{p}<0.001)$. There is, however, a tendency for an increase in the cell number of late-born neurons in the mutant mouse (especially for layer II/III cells) as shown previously (Polleux et al., 1998).

Remarkable differences were found in the cortical patterns of primary sensory areas located rostrally, medially and caudally in the reeler brain. Thus, in order to investigate them further, the relative depths of supragranular (Rgs8), granular (Rorb) and infragranular ( $\left.T C_{1460681}\right)$ fated- cells in M1 (rostral, Fig.10 A and B), S1BF (medial, Fig.10 C and D) and V1 (caudal, Fig.10 E and F) were analyzed and compared in detail. Results are shown in Figure 10. While the distribution of Rorb- neurons is restricted to a wide area in the middle of the adult cortex in all areas, cell distribution of early- $(R g s 8)$ and late- $\left(T C_{1460681}\right)$ born neurons are largely non-consistent along the rostro-caudal axis in reeler mice. $T C_{1460681^{-}}$neurons are split in deep and superficial "layers" in M1 (Fig.10 A and B - rl-/-) but appear to be piled up adjacent to the pial surface in V1 (Fig.10 E and F - rl-/-). Besides, the location of Rgs8- neurons in M1 (Fig.10 A and B - rl-/-) is superficial rather than deep, while in V1 (Fig.10 E and F - rl-/-) those cells reside deep in the inner half on the cortex.

Altogether the data show a clear evidence for the lack of one dominant cortical phenotype in the Reelin-deficient mice, but non-random and highly area-dependent (mis)lamination patterns. These cortical lamination patterns range from an intermingled, but more similar to the wild type phenotype in the rostral motor cortex, via an almost disappearance of layers in the intermediate somatosensory cortex, to a more inverted phenotype in the caudally located visual cortex. As a result, the patterns form an inside-out to an outside-in gradient through the anteriorposterior axis in reeler whose foundation has to be investigated further. As proper neocortical 
lamination relies on an appropriate cell production, correct neuronal migration and precise cell positioning during embryonic and early postnatal stages, those events were therefore inspected next.

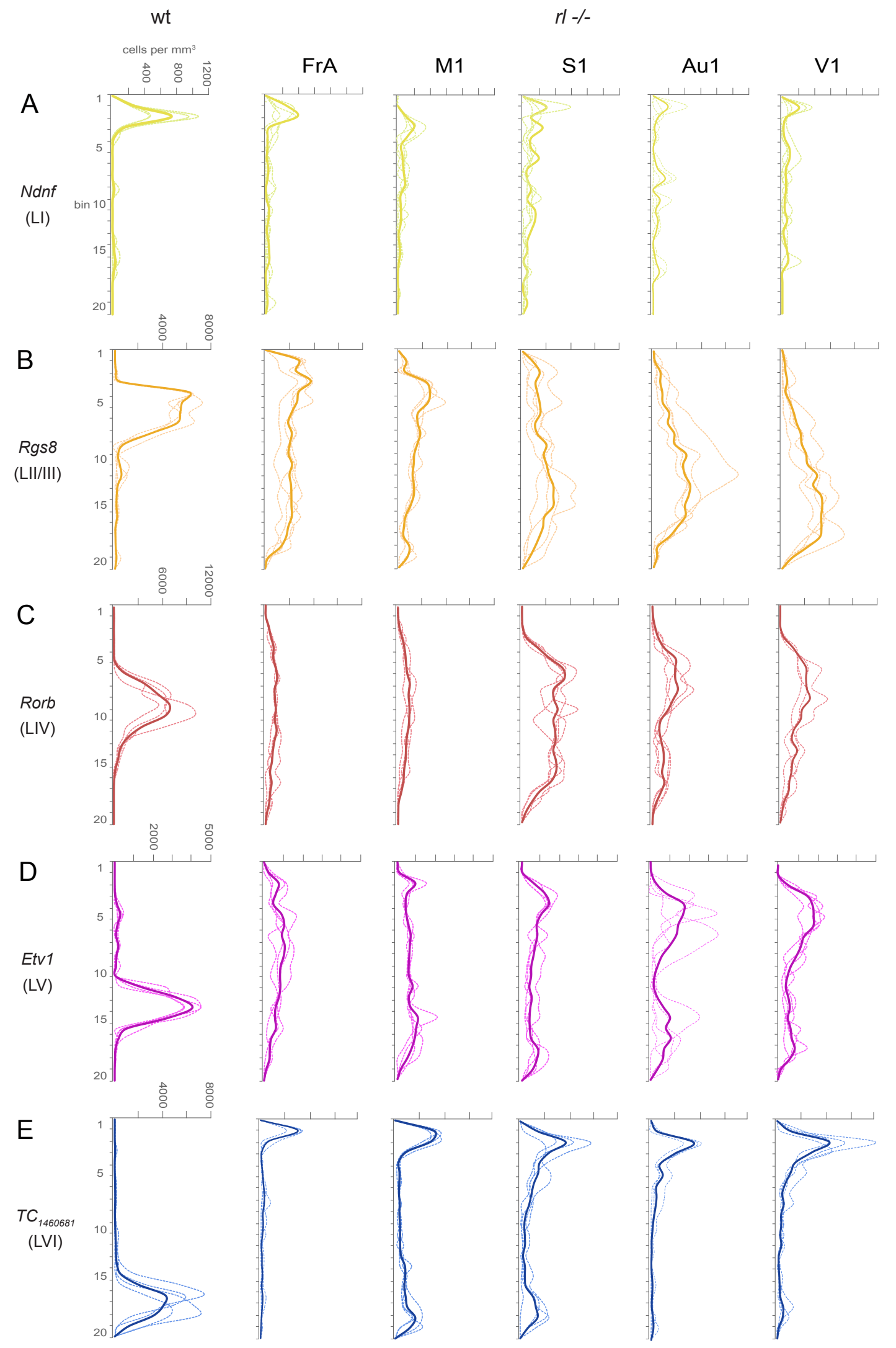


Figure 8. Radial distribution of layer-fate marker expressing neurons in a given cortical column in reeler. Spatial distribution (in cells per $\mathrm{mm}^{3}$ ) of $\mathrm{Ndnf}$ (LI; yellow), Rgs8 (LII/III; orange), Rorb (LIV; red), Etv1 (LV; purple) and $T C_{1460681}$ (LVI; blue) in the FrA, M1, S1BF, Au1 and V1 in rl-/- compared to representative density plots of a wt mouse (V1). Cell density is based on on-slide manual counting. Each cortical column was divided radially into 20 bins of equal size normalized to the height of the column. In the line charts, dark lines represent the average while faint lines correspond to the spatial distribution of the particular fate marker in each animal separately $(n=4)$. The location of the cells fated to the same layer is not random, as cell density distribution peaks in all animals at approximately the same bin range, specific for each cortical area and marker.

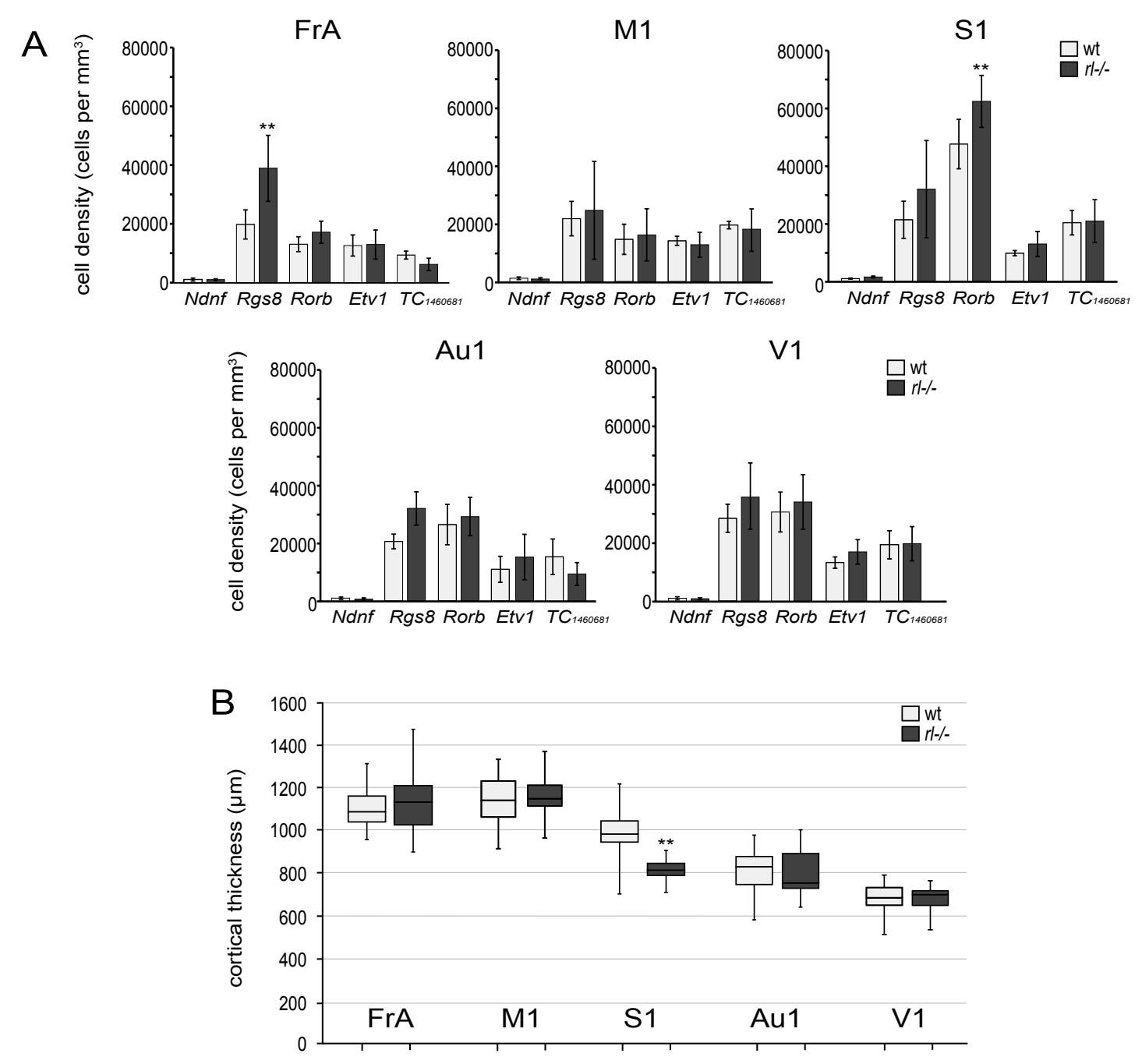

Figure 9. Cell density and cortical thickness in wild type and Reelin-deficient mice. Cell density (cells per $\mathrm{mm}^{3}$ ) of Ndnf- (LI), Rgs8- (LII/III), Rorb- (LIV; red), Etv1- (LV) and TC 1460681- (LVI) expressing cells in the FrA, M1, S1BF, Au1 and V1 (A) ( $n=4-6$ animals per lamina- fate marker and genotype) and cortical thickness (in $\mu \mathrm{m}$ ) of wt and $r l$-/- mice ( $n=20$ per cortical area and phenotype) (B). Neither cortical thickness nor cell density were significantly different when comparing the two genotypes, with singular exceptions. $\left({ }^{* *}\right) p<0.01$. 

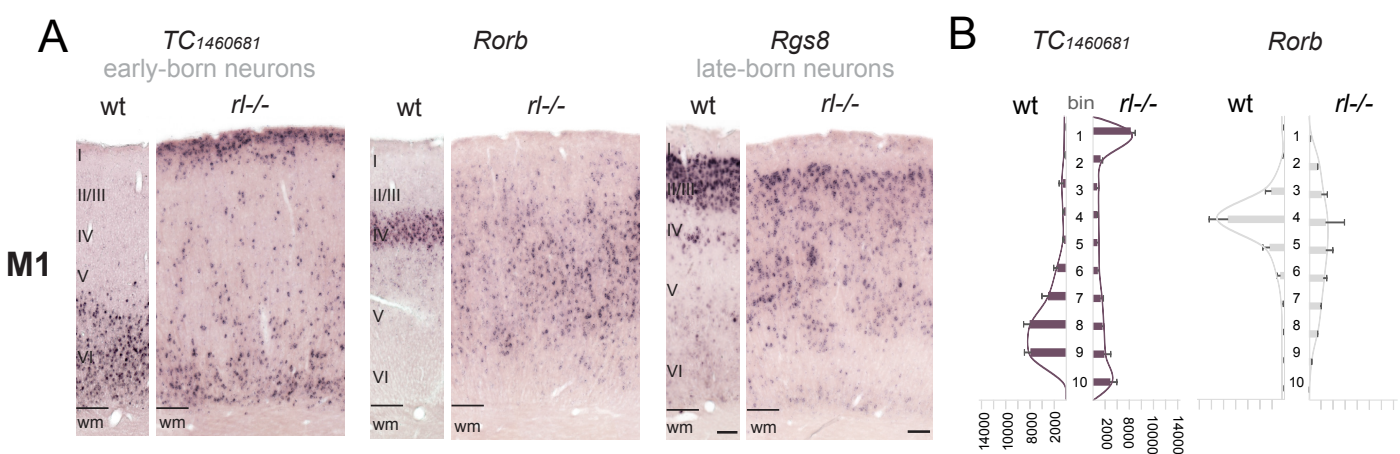

Rgs8

C
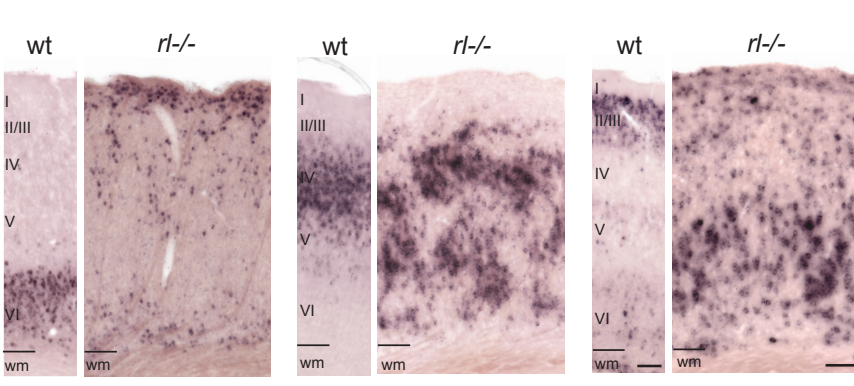

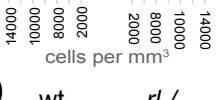

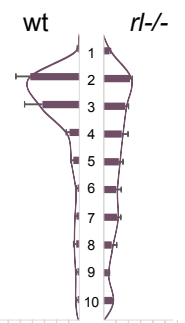

D w
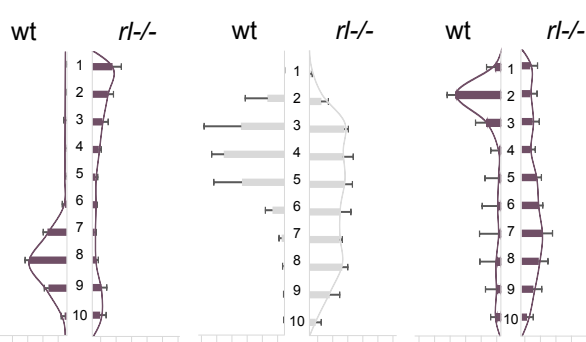

E
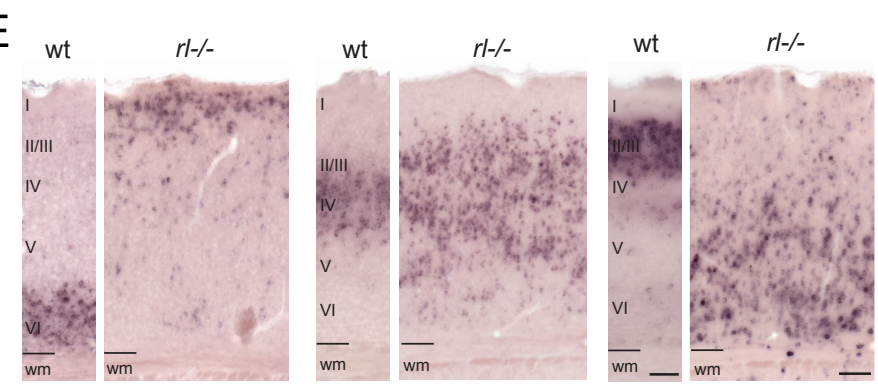

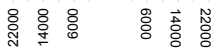

$\mathrm{F}$

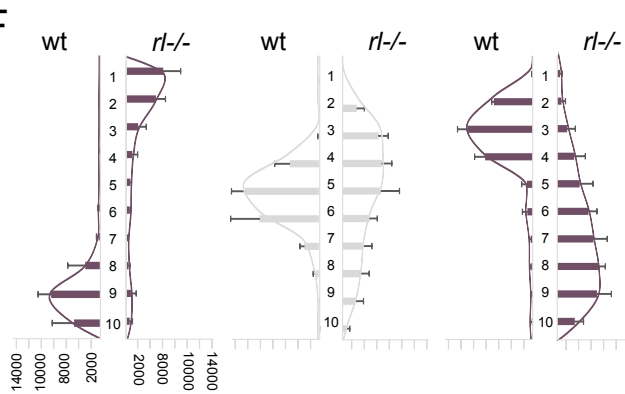

Figure 10. Cortical (mis)laminated patterns range from a quasi- inside-out in rostral sections, massive intermingling intermediately, to a quasi- inversion caudally. Chromogenic ISH for genes selectively delineating layer II/III (Rgs8), layer IV (Rorb), and layer VI (TC $1460681, \mathrm{C})$ in M1 (rostral - A), S1 (medial - C) and V1 (caudal -E) wt and $r$-/- sections. Comparison of the spatial arrangement of Rgs8-, Rorb- or $T C_{1460681^{-}}$expressing cells in M1 (B), S1 (D) and V1 (F) wt and $r$-/- cortices ( $n=4)$. Layers symbolized as Roman numerals. Each cortical column was divided radially into 10 bins of equal size, normalized to the height of the column. Cell density is represented as cells per $\mathrm{mm}^{3}$. Abbreviations: wm, white matter. Scale bars: $100 \mu \mathrm{m}$. 


\section{PREPLATE SPLITTING}

Neocortical development starts with the formation of a preplate. The preplate, or primordial plexiform layer, is found below the pia mater and it is composed of two pioneer populations of postmitotic neurons: the Cajal-Retzius (CR) cells and the subplate (SP) cells. The first cohort of migrating cortical neurons, the prospective layer VI cells (E13.5-born neurons), bypass the SP cells and form a layer, the early cortical plate, between the CR (superficially) and SP cells (below). Thus, they are considered to be responsible for splitting the preplate. Interestingly, the lamina-fate marker analysis in reeler showed major differences in the distribution of these cells (Fig. 7). In the mutant mouse, $T C_{1460681}$ - neurons are divided in two "layers" in M1 (rostral), while they are piled up in one "layer" adjacent to the pial surface in V1 (caudal). Therefore, and in order to investigate if the (mis)laminated patterns start by an area-specific defect at the first stages of corticogenesis, it was examined whether in reeler the preplate fails to split differently, in rostral and caudal cortices.

Due to the lack of good antibodies to efficiently label the components of the preplate, potential markers for $\mathrm{CR}$ and SP cells, whose expression can be detected only by in situ hybridization (ISH), were selected. CR and SP cells have multiple origin sites and highly dynamic genetic programs, which make the identification and tracking over development quite challenging. The selection of the candidates was done based on gene-expression profiles of CR and SP cells that became available just recently (Hanashima et al., 2007; Oeschger et al., 2012; Yamazaki et al., 2004). Thus, the Tumor suppressor gene (Trp73) and Early B-cell factor 2 (Ebf2) were used to identify CR cells. Candidates to discriminate SP cells were: Unc homologue $5 d$ (Unc5d), Cadherin 10 (Cdh10), T-plastin (P/s3), Cadherin 18 (Cdh18) and GABA-A receptor $\alpha 5$ (Gabra5). Chromogenic ISH (CISH) and fluorescence ISH (FISH) in wild type E15.5 cortices are shown in Figure 11. CISH provides a high- sensitivity labeling and offers the possibility of monitoring the enzymatic reaction at regular intervals, optimizing the signal to background ratio. It was, therefore, the method of choice to validate the expression of our candidates. CISH confirmed that Trp73 and Ebf2 (Fig.11 A and B, left) are specifically expressed in the marginal zone of the cerebral cortex, where CR cells reside. Potential SP-specific probes predominantly labeled cells in the subplate zone (C-G), with the exception of Unc5d, which was primarily expressed in the intermediate zone (IZ) (Fig.11 C, left), in disagreement with previous studies (Oeschger et al., 2012). Riboprobes were then tested by a tyramide signal amplified (TSA) FISH protocol. The sensitivity of the detection was not sufficient to visualize Ebf2, Cdh10, Cdh18 and Gabra5, 
probably due to a low mRNA concentration in the cells (Fig.11 B,D,F,G, right). However, moderateto-high abundance probes, like $\operatorname{Trp} 73$ (Fig.11 A, right) and Ps/3 (Fig.11 E, right), yielded a reliable and high-intensity labeling, and consequently were selected for the co-detection analysis.

Aiming to evaluate the completeness of the split of the preplate, CR and SP cells were visualized simultaneously by using a double FISH strategy. Thus, double FISH of Trp73 (as the CR cells marker) and Ps/3 (as the SP cells marker), was carried out at E15.5 on wild type and reeler rostral (M1) and caudal sections (V1) (n=3). Representative images are shown in Figure 12 A-C. In wild type sections, cortical plate neurons (stained with DAPI) have invaded the preplate, splitting it into the superficial marginal zone (Trp73- expressing cells, shown in pink - empty arrowheads), located nearby the pia mater, and the subplate (Pls3-expressing cells, shown in green - filled arrowheads), found above the intermediate zone (Fig.12 A,B,wt). In caudal reeler cortices, migrating neurons have failed to bypass the subplate cells, accumulating beneath the $P s / 3$ and Trp73-expressing zone (Fig.12 B, rl-/-). The cell position defect leads to the formation of an ectopic layer named superplate, as described previously (Caviness, 1982b) (Sheppard and Pearlman, 1997). However, in rostral cortices, cortical neurons seem to be able to move past their predecessors. Here, the location of Ps/3- expressing cells (filled arrowheads and dashed lined circles in Fig.12 A and C) is scattered through the upper-mid level of the cortical wall, and many presumptive cortical plate neurons (shown as DAPI cells) are located between the CR- and the dispersed SP- zone. This suggests that the preplate is partially diluted in rostral sections, while preplate splitting is prevented in caudal regions of the Reelin-deficient neocortex.

When thalamic axons reach the proper cortical region, approximately at day E15.5, fibers accumulate and rest in the subplate before continuing their growth (Catalano and Shatz, 1998; Molnar and Blakemore, 1995). Interestingly, afferent projections to the neocortex accompanies the cellular ectopia in reeler (Caviness, 1976; Simmons et al., 1982). The result from the double FISH suggested that preplate splitting is differentially disrupted over the rostro-caudal axis in the absence of Reelin. In order to support this hypothesis, the trajectories of thalamic fibers were investigated. Thalamic afferents were examined in ROR $\alpha$ animals (Fig.12 F-I). Here thalamocortical axons from neurons of the principal sensory thalamic nuclei express tdTomato under control of the ROR $\alpha^{\text {Cre }}$ reporter. In wild type animals, fibers target and rest in the area below the cortical plate, corresponding to the subplate zone (Fig.12 F-I, wt). ROR $\alpha$ reeler animals display a pattern in rostral cortices much closer to wild type, as many fibers target the same cortical depth, located below the developing cortical plate, as they do in the normal developing 
brain (Fig.12 F, rl-/-). However, a highly oblique fiber distribution, positioned next to the pia mater, can be observed in caudal sections (Fig.12 G, rl-/-). These distinctive fiber patterns are preserved at least until E17.5 (Fig.12 $\mathrm{H}$ and I, rl-/-). Thalamic fiber patterns were additionally examined by Calretinin immunostaining, which at the same time labels Cajal-Retzius cells (empty arrowheads) and thalamic projections (filled arrowheads), on E15.5 coronal sections (Fig.12 D-E). Altered routes of thalamic fibers, running up, obliquely oriented, through the entire cortex, were only obvious in caudal reeler cortices (Fig.12 E, rl-/-; filled arrowheads), indicating that the target cells (SP cells) are abnormally located above the cortical plate. However, rostral sections display a thalamic fiber trajectory more comparable to the wild type brain. In M1, despite few oblique afferents, fibers align parallel to the brain surface in the potential subplate zone, indicating that at least some SP cells must be located in lower cortical levels (Fig.12 D, rl-/-; filled arrowheads). The data thus show that, in the reeler neocortex, thalamic fibers preferentially target lower cortical levels in rostral cortices and the vicinity of the pia matter in caudal regions, indicating that SP cells are located below and above of the cortical plate, respectively.

Altogether, the results demonstrate that preplate splitting is not equally disrupted over the anterior-posterior axis of the Reelin-deficient neocortex. Thus, the partial preplate splitting in rostral areas and a compact superplate in caudal regions, might contribute to the differences in the spatial distribution of cortical neurons in $\mathrm{M} 1$ and $\mathrm{V} 1$ of the adult reeler mice.

Figure 11. Identification of molecular markers specific for Cajal-Retzius and subplate cells. Chromogenic ISH (CISH) and fluorescence ISH (FISH) for candidate genes to discriminate Cajal-Retzius (CR, A,B) and subplate (SP, $\mathrm{C}-\mathrm{G}$ ) cells in wild type E15.5 cortices. All probes were detected by $\mathrm{CISH}$ and their expression was restricted to $\mathrm{CR}$ (A, B) and SP (D-G) cells (arrowheads), with the exception of Unc5d. Trp73 and Ps/3 yielded a reliable and high intensity labeling by FISH $(A, E)$ and were therefore selected for the co-expression analysis. Abbreviations: $c p$, cortical plate; iz, intermediate zone; vz, ventricular zone. Scale bars: $100 \mu \mathrm{m}$. 

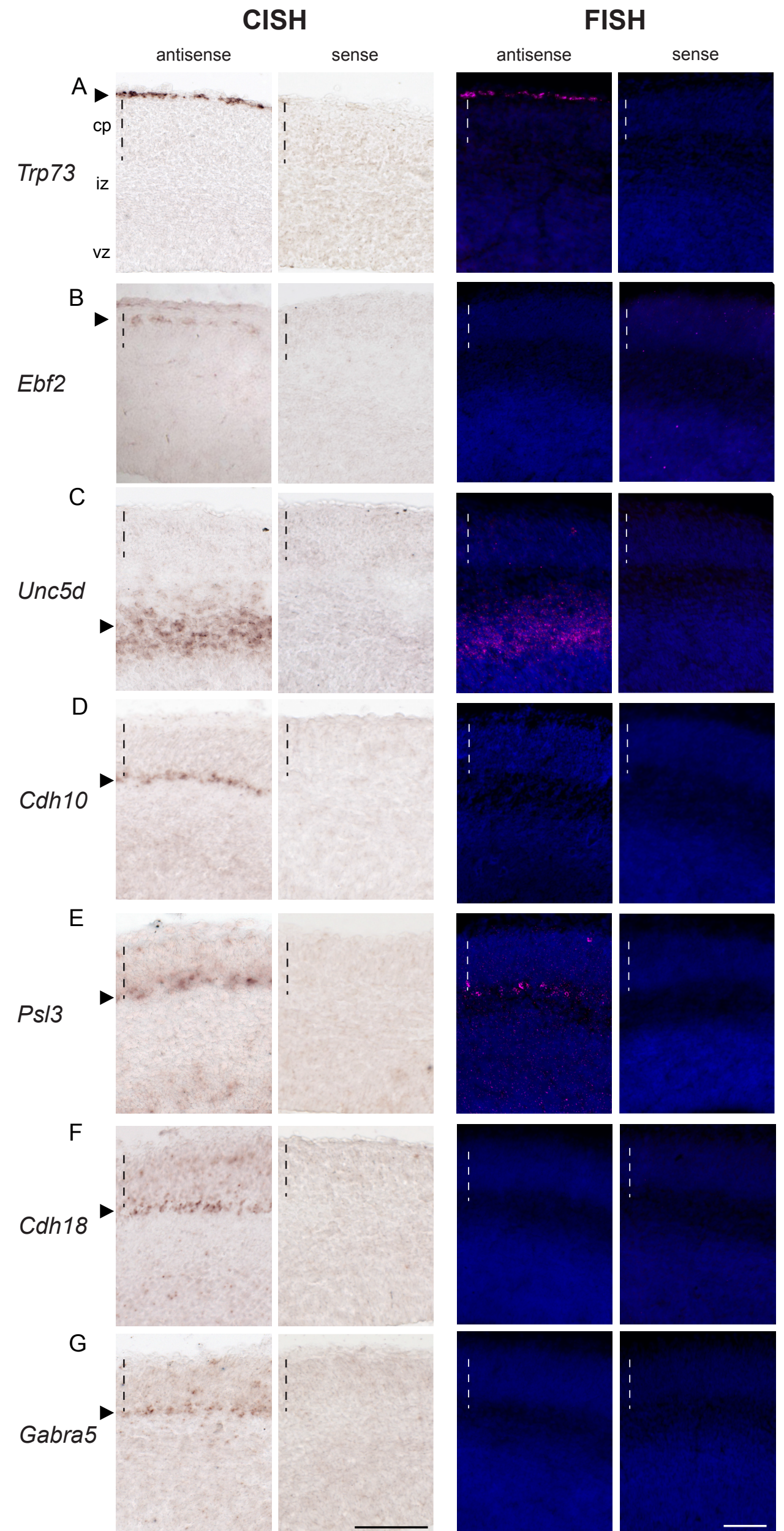

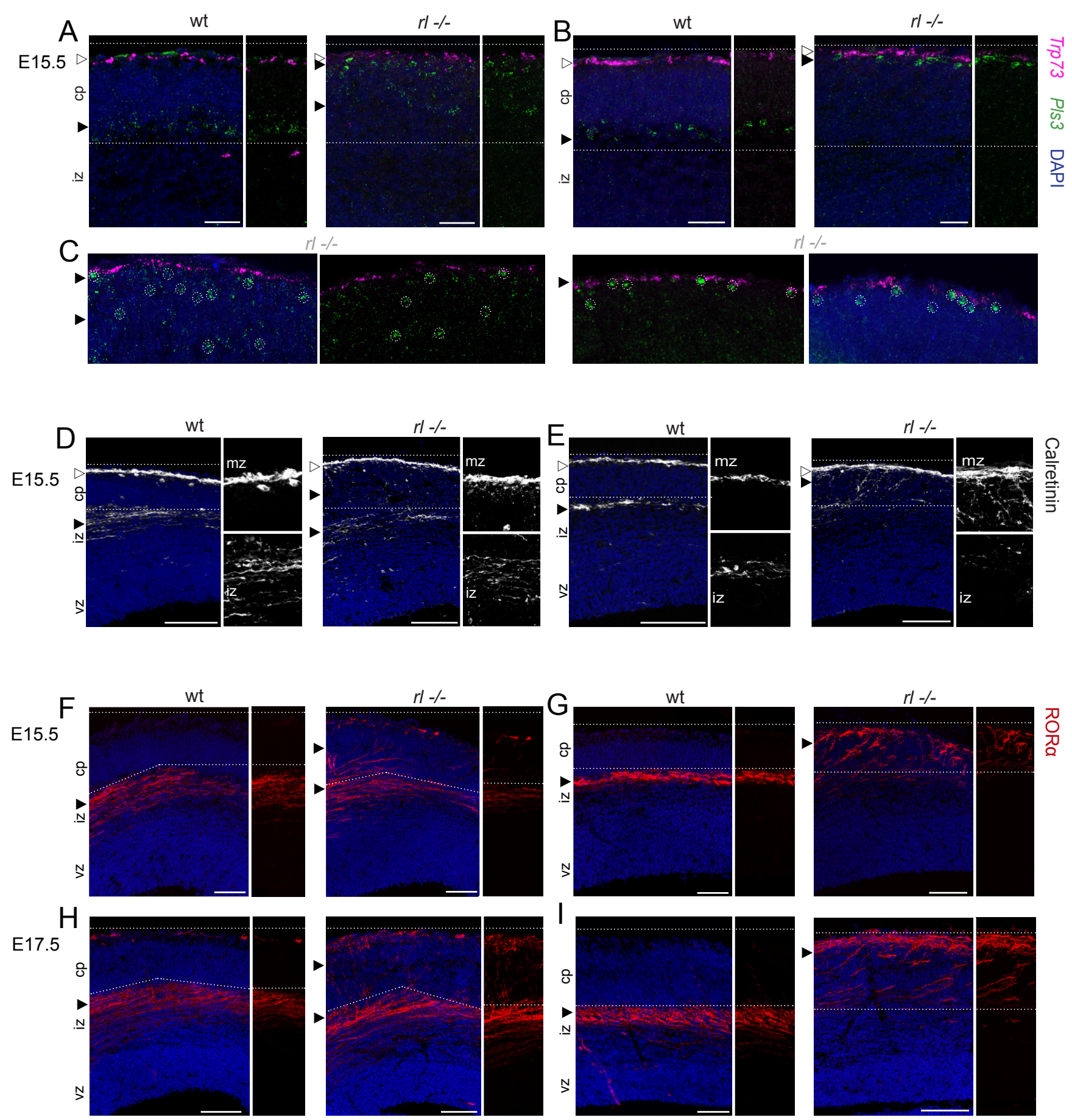

Figure 12. Preplate splitting is altered in rostral areas but does not occur in caudal regions. Expression of $\operatorname{Trp} 73$ (CR cells) and PIs3 (SP cells) were detected by double fluorescence in situ hybridizations on rostral and caudal E15.5 wt and $r$-/- cortices (A,B,C). Marginal zone (empty arrowheads), SP cells and thalamic fibers (filled arrowheads). Migrating neurons in the wt cortex split the preplate (Sheppard and Pearlman, 1997) into the superficial marginal zone (shown in pink) and the deeper subplate (shown in green). In contrast, in caudal reeler 
sections, neurons expressing SP specific genes remain at the top of the cortical wall, forming an ectopic layer termed superplate, while a partial preplate splitting can be seen in rostral cortices. E15.5 Calretinin (D,E) and E15.5 and E17.5 RFP (ROR $\alpha^{\text {tdTomato }}$ mouse line) labelling (F-I). In reeler M1, despite few oblique afferents, fibers align parallel to the brain surface targeting the potential subplate zone. In reeler $\mathrm{V} 1$, however, fibers are obliquely oriented indicating that the target cells (SP cells) are abnormally located above the cortical plate. Abbreviations: $c p$, cortical plate; iz, intermediate zone; vz, ventricular zone ; $\mathrm{mz}$, marginal zone. Scale bars: $100 \mu \mathrm{m}$.

\section{NEOCORTICAL HISTOGENESIS OF THE REELIN-DEFICIENT BRAIN DURING BRAIN DEVELOPMENT}

The analysis of the lamina- fate markers in the adult neocortex revealed a vast range of lamination entropy in reeler. This entropy is, however, not random. Lamination patters in primary cortices form an inside-out (M1) to outside-in (V1) gradient through the anterior-posterior axis in reeler. Although rostro-caudal differences in preplate splitting may contribute to the adult lamination phenotypes, how those patterns are formed is still a question. Disturb migration is a likely candidate mechanism.

As cortical layering is associated to neuronal migration, migration and cell positioning were examined next, over the anterior-posterior brain axis of the reeler brain. The thymidine analog labeling approach have conquered the studies of cell proliferation, cell birth-dating and neuronal migration during brain development (Angevine and Sidman, 1961; Azim et al., 2009; Cavanagh et al., 2011; Caviness, 1982a; Caviness and Sidman, 1973; Hack et al., 2007b; Polleux et al., 1998), and was therefore selected as the first method of choice. Thymidine analogues incorporate into the de-novo synthesized DNA in the S-phase (DNA replication) of dividing cells. Cells that exit the cell cycle and become postmitotic directly after the injection are strongly marked by the thymidine analogue injected at that time. Those that re-enter the cell cycle subsequently dilute the tag through serial rounds of mitosis, as the DNA of the progenitor cells is distributed (to $50 \%$ ) to the two resulting daughter cells. Although still under debate (Gao et al., 2014; Hevner et al., 2003a; McConnell and Kaznowski, 1991; Nieto et al., 2004), it is believed that neocortical projection neurons that share birth dates, have the same cell and layer fate. Therefore, and as the nuclei of the cells are permanently labeled by this method, one can determine the birth dates of homogenous neuronal cohorts, compare the relative positions of those neurons that arise at the same developmental time, and explore migration and layer formation kinetics over brain development. Moreover, by separating the administration of 
nucleoside analogues at given intervals, in the same animal, discrimination of cell populations is possible.

Current protocols use one or two sequential pulses of the chosen thymidine analog(s) available. In such protocols, cell tracing is restricted to two cohorts of cortical neurons. However, the neocortex has six layers, shaped by six distinct cohorts of projection neurons. The neocortex can also be broadly divided into three main compartments: supragranular, granular and infragranular layers. Thus, to more thoroughly visualize this cellular diversity, we designed a new method, based on the sequential administration of three different types of thymidine analogs, by which one could trace simultaneously cells fated to those sub-regions. In summary, pulse labeling (by acute intraperitoneal injections) of modified thymidine analogues was performed at E13.5 (5ethynyl-2'-deoxyuridine, EdU), E14.5 (5-chloro-2'-deoxyuridine, CldU) and E15.5 (5-iodo-2'deoxyuridine, IdU), in order to predominantly label infragranular, granular and supragranular cortical layers respectively. Differentially tagged progeny were discriminated with an antibodybased (CldU, IdU) or chemical-based (EdU) detection. Labelling principle is shown in Figures 13 and 14. The strategy uses two anti-BrdU antisera generated in different species (mouse and rat) with different binding affinity to CldU and IdU, extensively used and tested in previous studies (Azim et al., 2009; Teta et al., 2007; Vega and Peterson, 2005). After validation, this method was used to investigate the chronology of neuronal migration and layer formation in a wild type versus a Reelin-lacking environment.

\section{(Cell) birth dating labeling principle}

Layer position and cell fate identity was first evaluated at postnatal stages. Analysis at P7, when migration is known to be completed, showed that cells labeled with EdU (born at E13.5, shown in magenta), CldU (born at E14.5, shown in green) and IdU (born at E15.5, shown in red) reside indeed predominantly in infragranular, granular and supragranular layers respectively (Fig.13 B). The phenotypic identity of the cells was verified by using established molecular markers for layerrestricted neuronal subpopulations (Fig.13 C-F). A minor fraction of E13.5-born cells show immunoreactivity for Ctip2, a layer V cell maker $(2.77 \% \pm 0.81)$, Cux1, a well-known marker for layer II/III $(1.22 \% \pm 1.33)$ or both $(0.56 \% \pm 0.26)$. On the contrary, a vast number of E15.5-born cells also expressed Cux1 $(75.58 \% \pm 2.85$ out of the total IdU labeled cells). It could be therefore 
concluded that the administration of EdU at E13.5 leads to the labeling of lower layers, while the injection of IdU at E15.5 results in the labeling of upper layer neurons.

The triple-injection strategy is also suitable to trace cohorts of migrating neurons at embryonic stages (Fig.14). The delamination from the ventricular zone and the migration of projection neurons follows the sequence by which neurons are generated. Thus, when cortical lamination is analyzed at late stages of embryonic development, early-born neurons are expected to have traveled further in comparison to late-born neurons. And that is exactly what we saw in tripled-injected animals. At E17.5, when neurogenesis in the neocortex is coming to its end and the cortical plate is formed, E13.5-born cells have reached the cortical plate (CP), E14.5-born cells reside in the intermediate zone (IZ), or have already bypassed the early-born neurons population, and E15.5-born cells are mainly found below, in the ventricular-intermediate zone (VZ-IZ) (Fig.14 $A, C)$. The administration of nucleoside analogs at the designated intervals lead to a clear separation of cell populations, as single $\left(\mathrm{EdU}^{+}, \mathrm{CdlU}^{+}, \mathrm{IdU}{ }^{+}\right)$, double $\left(\mathrm{EdU}^{+} / \mathrm{CldU}^{+}, \mathrm{EdU}^{+} / \mathrm{IdU}^{+}\right.$, $\left.\mathrm{CldU}^{+} / \mathrm{IdU}^{+}\right)$and triple $\left(\mathrm{EdU}^{+} / \mathrm{CldU}^{+} / \mathrm{IdU}^{+}\right)$labeled cells can be even identified in a section on its peak of neuronal migration (Fig.14 B,D). These labeling combinations provide a unique tag that can be used to specify when a neuron was born during development.

The fact that halogenated uridines are diluted by successive cell-cycle re-entries, although useful for proliferation assays, complicates a reasonable analysis in a system at the top of its expansion with three varieties of thymidine analogs in the environment. Therefore, and based on the semi-conservative DNA replication law, analysis by ImageJ and RStudio was carried out in cells with a labeling intensity greater than $50 \%$ of the maximum labeling (the analysis was normalized to the properties of each section). These cells have exited the cell cycle right after the pulse and can be, therefore, considered the first progeny of a progenitor cell in a given period. Violin plots based on this principle, corresponding to images in Fig.14 A, and proportions of single, double and triple labeled cells are shown in Fig.14 C and Fig.14 D respectively. Plots faithfully reflect the progression of cortical development, since early-born neurons $\left(\mathrm{EdU}^{+}\right)$have migrated further distances while late-born neurons (e.g. $\mathrm{IdU}^{+}$) are still on their way, as described above. Analysis of the $50 \%$ - brightest population gives a high percentage of single labeled cells (> $80 \%$ ) with a minor fraction of co-expressing neurons. Based on the data, the analysis of the thymidine analog injected pups was then focused on the 50\%- brightest neurons in the following experiments, unless stated otherwise. 
A

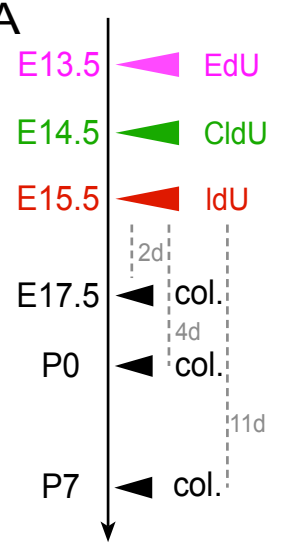

C

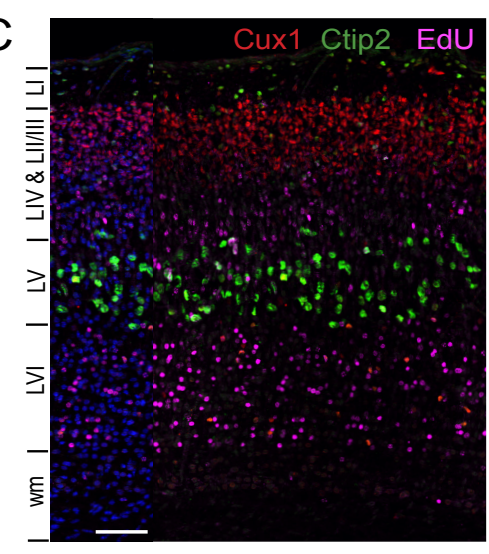

$\mathrm{E}$

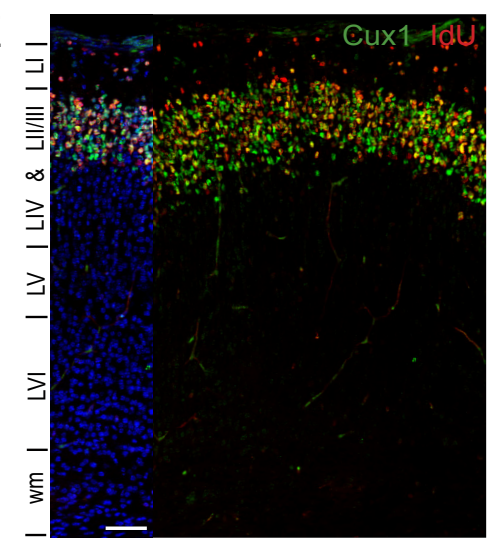

$\mathrm{B}$
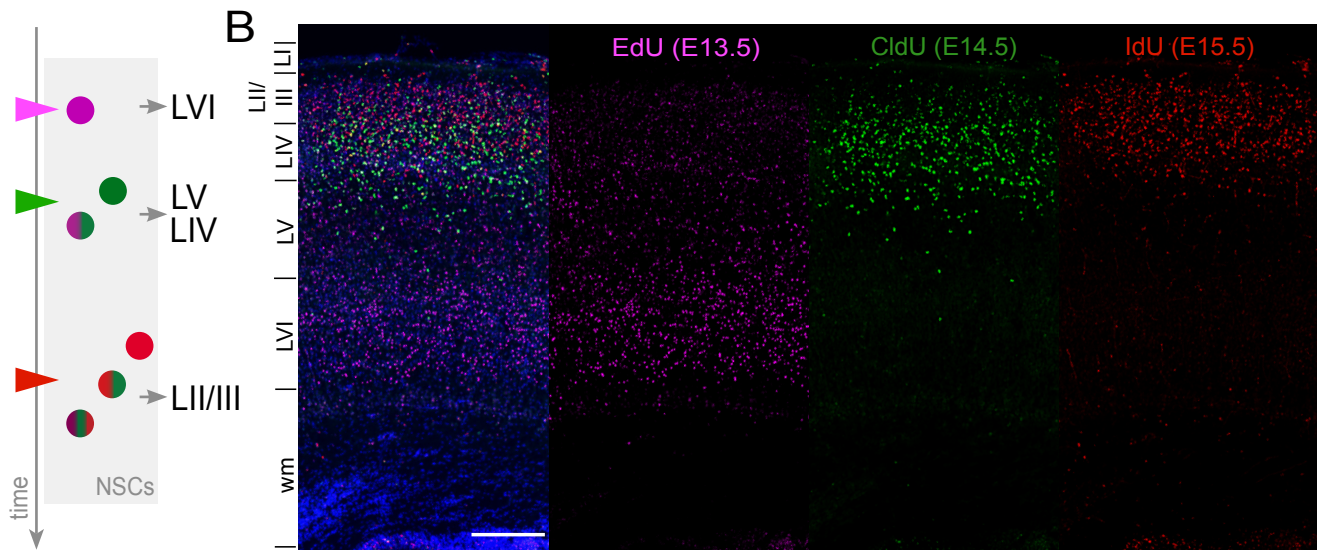

$\mathrm{D}$
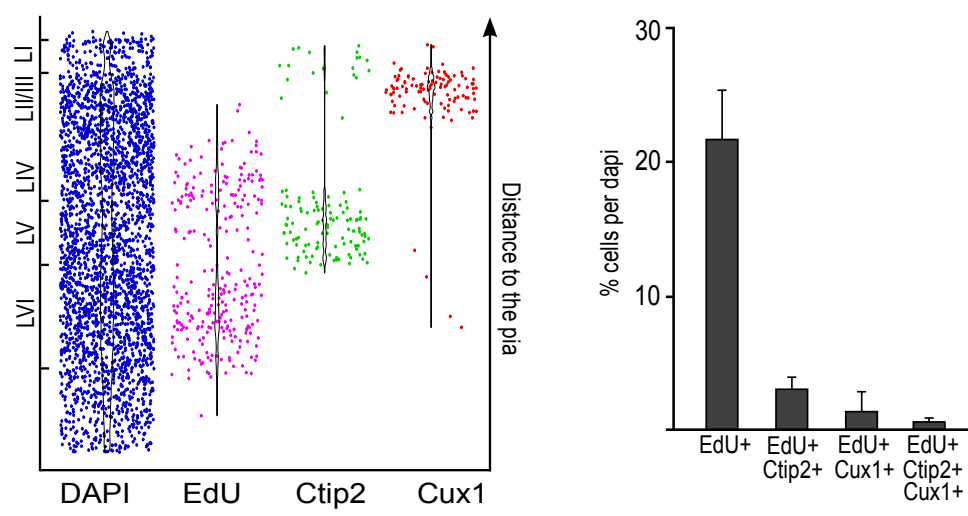

$\mathrm{F}$

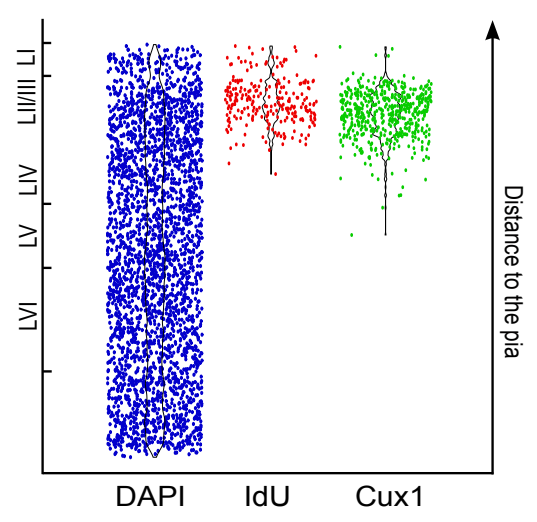

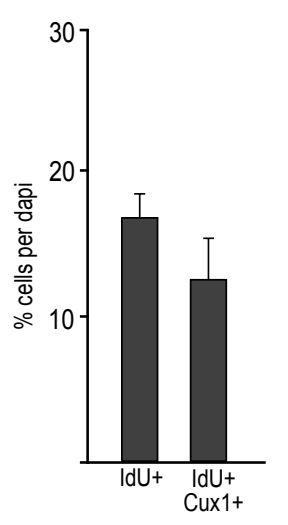

Figure 13. Cell birth dating labeling principle (I). Experimental design (A) and labeling principle (B). Injections (i.p.) of thymidine analogues were done at E13.5 (EdU, magenta), E14.5 (CldU, green) and E15.5 (IdU, red) in order to label infragranular, granular and supragranular layers, respectively. Immunostaining against the different analogues in a wild type P7 coronal section (B) show that indeed EdU+, CldU+ and IdU+ cells reside predominantly in lower-, mid- and upper- layers. The fate of the labeled cells was resolved by using markers for lineage commitment (C to F). Violin plots represent distribution of total (DAPI) or 50\%- brightest (EdU, Ctip2, Cux1 and IdU) cells in C (D) and E (F). Single and co-labeling quantification shown as percentage of cells per DAPI in wild type P0 coronal sections ( $n=4$ ) ( $D$ and F). Abbreviations: wm, white matter. Scale bars: $200 \mu \mathrm{m}$ (B) and 50 $\mu \mathrm{m}(\mathrm{C}, \mathrm{E})$. 

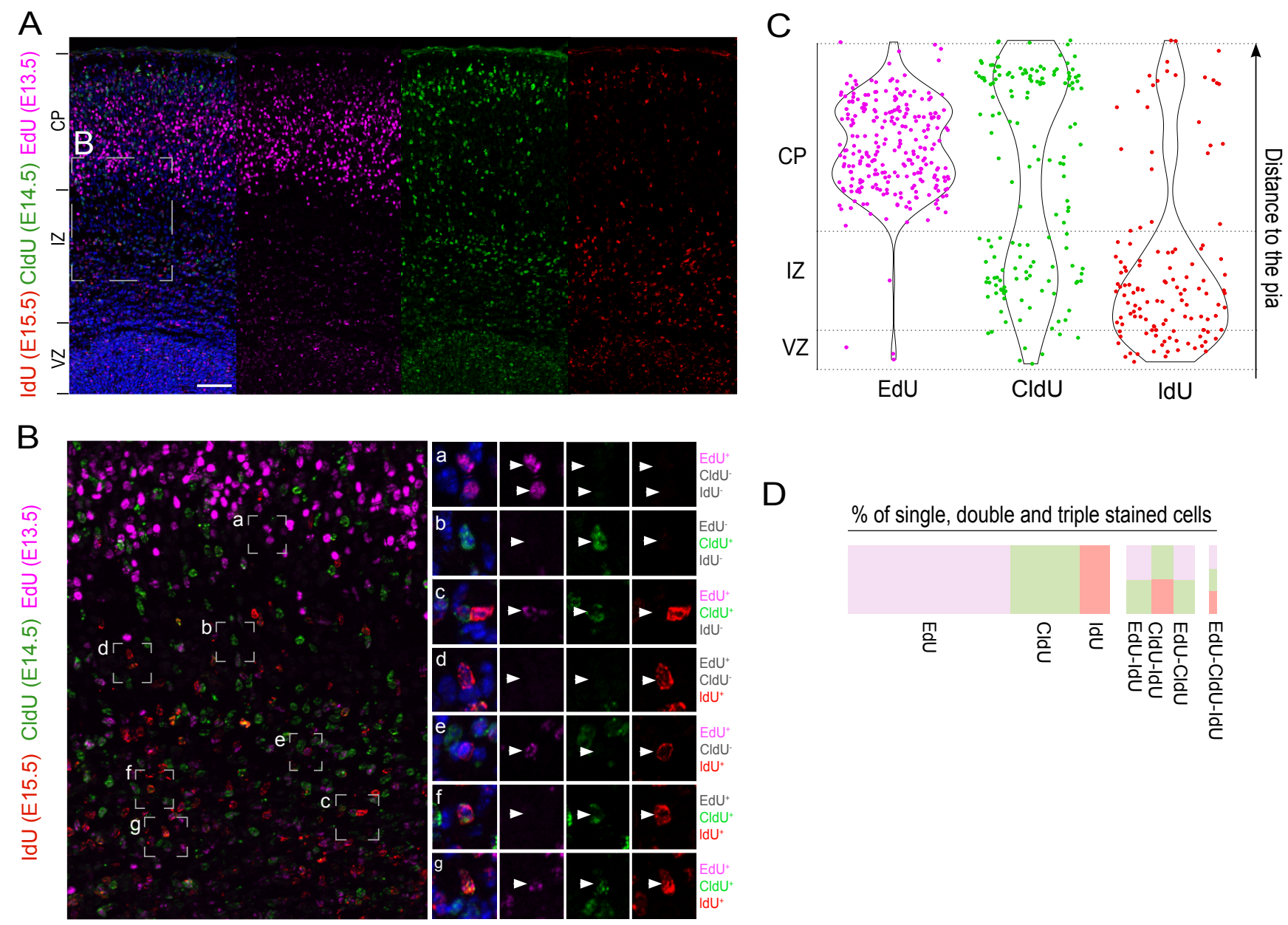

Figure 14. Cell birth dating labeling principle (II). Injections (i.p.) of thymidine analogues were done at E13.5 (EdU, magenta), E14.5 (CldU, green) and E15.5 (IdU, red) in order to label infragranular, granular and supragranular layers respectively. Immunostaining against the different analogues in a wild type E17.5 coronal section $(A)$ revealed the location of migrating neurons that included the modified nucleotides, where a clear discrimination of the birth-dated labelled populations is possible. Violin plots represent the distribution of the $50 \%$ - brightest population of each thymidine analogue in the cortical column shown in $A(C)$, being more than $80 \%$ of the cells single-labeled. The staining procedure is suitable for the detection of single $(B, a-C)$, double (B, d-f) and triple (B, g) label co-localizations. Percentage of single-, double- and triple- stained cells after thresholding for the 50\%- brightest labeled cells (x-axis=0 to 100\%) (D). Abbreviations: cp, cortical plate; iz, intermediate zone; vz, ventricular zone. Scale bar: $100 \mu \mathrm{m}$. 


\section{Progression of cortical (de)lamination in the S1BF}

The development of cell positioning of infragranular, granular and supragranular fated cells was first examined in the S1BF of wild type and reeler brains. Injected animals were harvested at the end of neurogenesis (E17.5), late stages of cortical migration (PO) and once neuronal migration was completed (P7) (Fig.15), thus covering the whole migration process.

At E17.5 (Fig.15 A, D), E13.5-born neurons (EdU ${ }^{+}$, shown in magenta) in the reeler phenotype have reached the $\mathrm{CP}$, as in their wild type counterparts. However, a substantial number of cells have not entered the $\mathrm{CP}$ yet. Cells born a day after ( $\mathrm{CldU}^{+}$, shown in green) show a clear delay and are mainly located in the IZ and the lower CP in reeler (Fig.15 A, arrowheads). In contrast, some of these E14.5-born cells in wild type are already located above the EdU-zone. This indicates that these cells are already bypassing early-born neurons in wild type, while they mostly fail to do so in reeler. Neuronal migration delays might lead to the diffuse boundaries between the $\mathrm{CP}$ and the $\mathrm{IZ}$ seen in the mutant mouse. E15.5-born neurons (IdU ${ }^{+}$, shown in red) are mostly in the IZ-VZ neighborhood, in both conditions.

Two days later (Fig.15 B, E), wild type cortices from P0 animals displayed a normal relative positions of tagged neurons, following the well described birth-dependent inside-out pattern. Thus, E13.5-born cells generate deep layer neurons, while E15.5-born cells, which constitute the future layer II/III, are located superficially, bypassing the E14.5-born population. On the contrary, the Reelin-deficient neocortex shows a massive migration delay of E14.5- and E15.5- born cells, as the great majority of these cells are still confined to lower cortical levels (Fig.15 B, arrowheads rl-/-).

At P7 (Fig.15 C, F), when migration is known to be completed, reeler fails to form any structure that resembles a layer. Instead, it displays a substantial cell intermingling, mirroring the adult (mis)lamination pattern. 


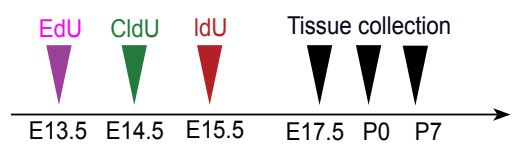

E17.5

wt
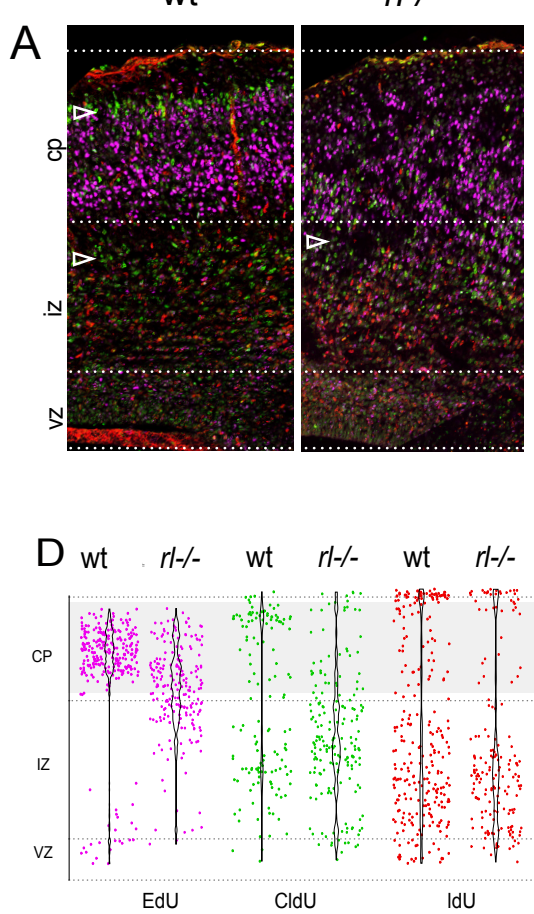
rl-/-

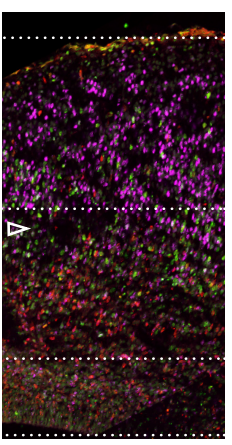

P0
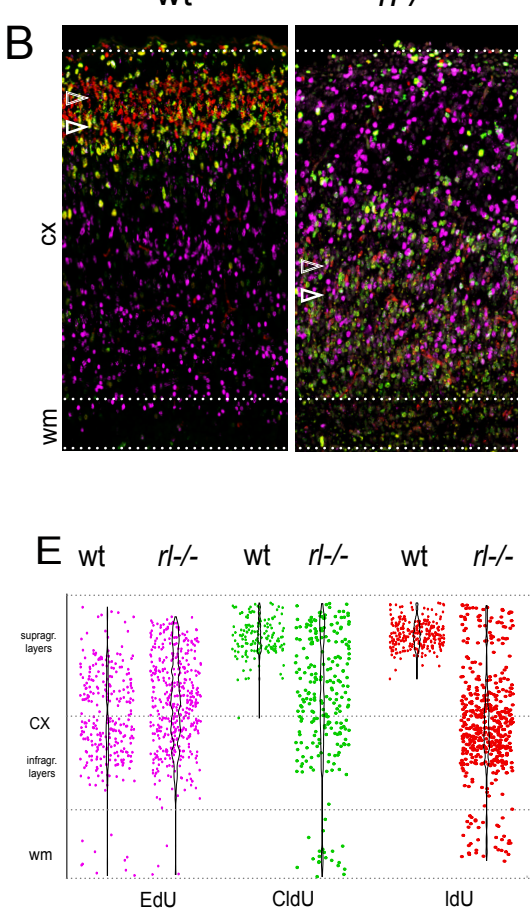

P7

wt

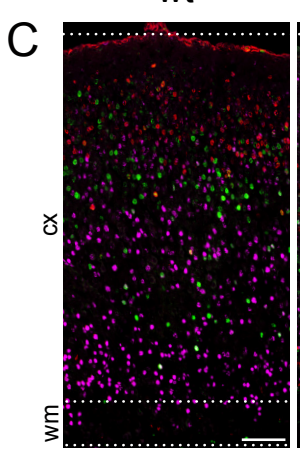

rl-/-
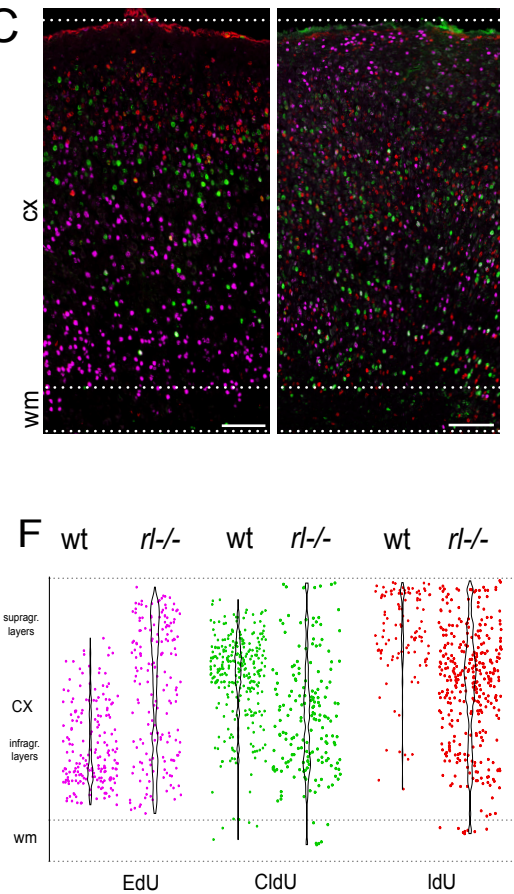

Figure 15. Development of cortical (de)lamination in the S1BF of reeler mice. Representative coronal sections of E17.5, PO and P7 injected wt and rl-/- mice (A-C) and the corresponding cell distribution in the developing cortical column (D-F). At E17.5, E13.5-born neurons (EdU', shown in magenta) reside superficially and E15.5-born neurons ( $\mathrm{IdU}^{+}$, shown in red) in both conditions are mainly restricted to the IZ. Granular cells (CldU ${ }^{+}$, shown in green) show a distinct delay upon reaching the cortical plate (A, D). From P0 to P7 neurons progressively settle down into the designated layer in wild type (B-F). The reeler cortex displays a massive E14.5- and E15.5- born cells delay at $P O(B, E)$. At $P 7$, the mutant mouse fails to constitute any resemblance of a layer and culminates in shaping a highly intermingled S1BF cortex (C, F). Arrowheads in (A) refer to CldU, and in (B) to CldU and IdU. Cortical plate $(D)$ is shaded. Abbreviations: $c p$, cortical plate; iz, intermediate zone; vz, ventricular zone; $c x$, cortex; wm, white matter. Scale bars: $100 \mu \mathrm{m}$. 


\section{Behavior of early-born neurons}

By detecting $T C_{1460681}$, which expression is restricted to layer VI cells, we could demonstrate that cell distribution ranges from two virtual layers in $M 1$, towards a drastic inversion in V1 (Fig.10). But how does it develop? Relative locations of E13.5-born cells (EdU') were examined in $\mathrm{M} 1$ (rostral), S1 (medial) and $\mathrm{V} 1$ (caudal) cortices, at E17.5, P0 and P7, in both genotypes. Representative results are shown as violin plots in Figure 16. At E17.5 (Fig.16 A), earlyborn cells were almost restricted to the $\mathrm{CP}$ in all wild type and reeler cortical areas, with the exception of reeler S1 (where cells seems to have a little delay). Reeler cortices showed, however, greater cell dispersion. At PO (Fig.16 B), most of the early-born cells reside in lower layers in the normal condition. In contrast, in Reelin-deficient sections, a similar cell dispersion, from top to bottom of the cortex, is obvious in M1, S1 and V1. At P7 (Fig.16 C), the relative cell location remains hardly unchanged in wild type cortices, as the location of the early-born cells is still restricted to lower layers. In reeler, despite a great cell scattering, cell location peaks in two "layers" in M1, next to the pial surface and in the vicinity of the white matter, while they are preferentially located in upper cortical levels in V1. In summary, an obvious transformation, from a common cell scattering towards a preferential cortical location, happens between P0 and P7 in reeler. Interestingly, it corresponds to the time in which late-born neurons get to their designated final cortical location.

\section{Infragranular vs. supragranular cell distribution}

The layer-fate marker analysis showed that, under the same Reelin-deficient environment, M1 and V1 show opposing laminated patterns in adult animals (Fig.4 $\mathrm{D}^{\prime}$ and $\mathrm{J}^{\prime}$ ). While the distribution of layer IV is restricted to mid-cortical levels in both areas, the distribution of layer VI and layer II/III fated cells is especially striking (Fig.10 A, B and E, F Rgs8 and TC 1460681 ). To elucidate how these two patterns are shaped, infragranular and supragranular layers were labeled by EdU (at E13.5, shown in magenta) and IdU (at E15.5, shown in green), respectively. Embryos were harvested on E17.5, when neurons are still migrating, and P7, when migration is over (Fig.17). To facilitate the analysis, the cortical wall was divided into 20 bins of equal size. Cortex (CX), cortical plate (CP), intermediate zone (IZ) and ventricular zone (VZ) were delineated according to the DAPI staining.

Cell density. We first asked whether cell proliferation differs in the normal and mutant condition. Data shows that, at least for the generation of cells born directly after the injections, there are no significant differences in cell density (in $\mathrm{mm}^{3}$ ) of EdU (early-born) or IdU (late-born) 
labeled cells between wild type and reeler cortices, neither in rostral nor in caudal areas (E17.5, $\left.\mathrm{n}=3-6 ; \mathrm{p}\left(E d U_{\text {rostral }}\right)=0.16, \mathrm{p}\left(I d U_{\text {rostral }}\right): 0.63, \mathrm{p}\left(E d U_{\text {caudal }}\right)=0.61, \mathrm{p}\left(/ d U_{\text {rostral }}\right): 0.33\right)$ (Fig.17 B).

In addition, the examination of the progenitor pool at E15.5 (Fig.18) indicates that there are no substantial differences in the number of $\mathrm{Pax6}^{+}$(RGCs in the VZ) or $\mathrm{Tbr}^{+}$(IPs in the SVZ) cells in anterior or posterior regions of wt and reeler mice $\left(n=3, p\left(\right.\right.$ Pax $\left._{\text {rostral }}\right)=0.056, p\left(\right.$ Tbr $\left.2_{\text {rostral }}\right): 0.61$, $\mathrm{p}\left(\right.$ Pax6 $\left._{\text {caudal }}\right)=0.66, \mathrm{p}\left(\right.$ Tbr2 $\left.\left.\left._{\text {caudal }}\right): 0.68\right)\right)$.

Thus, the number of neurons that became postmitotic after the nucleoside analog administration appears to be normal in M1 and V1 in reeler. And so does the progenitor pool. They can be therefore excluded as responsible for the divergent laminated phenotypes, as suggested in a previous study (Caviness and Sidman, 1973).

Cell position. As neurogenesis seemed not to be affected, we next examined the relative positions of early- and late- born neurons at two key points for neuronal migration and lamination development: E17.5 and P7. At late stages of corticogenesis (E17.5), when neurons are still migrating and the neocortex is still "work in progress", cells with shared birth date migrate corresponding to the sequence they are born. Consequently, E13.5-born cells (EdU ${ }^{+}$, magenta) reside superficially and E15.5-born cells ( $\mathrm{IdU}^{+}$, green) are found below, in anterior and posterior regions in both genotypes (Fig. $17 \mathrm{C}-\mathrm{F}$ ). Approximately $90 \%$ of the E13.5-born cells have already settled down in the CP in wild type and reeler ( $n=4-6)$ (bins 1-10: $E d U w t_{\text {rostral }}=90.17 \% \pm 13.92, E d U$ $r l-/$ rostral $=86.41 \% \pm 11.7, E d U w t_{\text {caudal }}=95.49 \% \pm 2.28, E d U$ rl-/- $\left.{ }_{\text {caudal }}=94.02 \% \pm 8.52\right)$. Notice that the location of these cells is already restricted to lower cortical levels in wild type. In contrast, reeler shows a pronounced cell dispersion (as described above), with early-born neurons slightly shifted towards upper cortical levels in caudal cortices ( $E d U$ rl-/- bins 2-6 $=51.32 \% \pm 7.66, E d U$ rl-/- bins 7 ${ }_{10}=34.11 \% \pm 13.88$ ). The vast majority of late-born neurons (E15.5-born cells) are found from bins 11-20, corresponding to the IZ-VZ region, in wild type and mutant mice (bins 11-20: IdU wt rostral $=77.22 \% \pm 13.92, \quad I d U$ rl-/- rostral $=86.41 \% \pm 11.7, \quad$ IdU wt caudal $=95.49 \% \pm 2.28, \quad$ Id $U$ rl-/caudal $=94.02 \% \pm 8.52$ ).

At P7 when migration has finished, early-born neurons reside in lower layers while lateborn neurons occupy upper layers in wild type sections (e.g. bins 9-20: EdU wt rostral $=66.17 \% \pm 16.46$, bins $2-7: I d U w t$ rostral $=81.99 \% \pm 15.73$ ). The Reelin-deficient neocortex fails to acquire this mature pattern, in both rostral and caudal regions (Fig. $17 \mathrm{G}-J$ ). However, although reeler cortices show a pronounced scattering of all labeled neurons, there are obvious differences between M1 and V1. E13.5-born cells constitute the silhouette of two "layers" in rostral sections (bins 1-6: $E d U \mathrm{rl}-/$-rostral $=17.11 \% \pm 5.21$, and bins 15-20: $E d U \mathrm{rl} / \mathrm{/}$ - rostral $=43.92 \% \pm 2.97$ ), while they 
are preferentially located in upper cortical levels in caudal cortices (bins 1-10: EdU rl-/cauda $=63.81 \% \pm 7.072$ ). Moreover, cells fated for upper layers (late-born cells) have indeed reached the upper cortex in rostral sections, bypassing a large number of their predecessors (bins 1-10: $I d U \mathrm{rl} / /$ - rostral $=51.37 \% \pm 11.52)$. On the contrary, these cells are clearly restricted to lower layers and accumulate below the early-born neuronal population zone in caudal cortices of the mutant mouse (bins 10-20: IdU rl-/- caudal $=77.34 \% \pm 9.01$ ).

The results indicate that delamination from the ventricular zone happens normally and neurons migrate according to the sequence they are generated in reeler. Thus, cells that were born later are migrating behind from those born previously. It was also shown that, when analyzed at late embryonic stages, E13.5-born cells have already reached the cortex and E15.5born cells are located in the IZ in rostral and caudal regions, as in the wild type littermates. However, very distinct distribution of early- and late- born neurons is seen when migration is completed (P7). These patterns mirror the cell distribution seen in the M1 and V1 cortices in adult animals. It is believed that cortical projection neurons in reeler are not capable to reach their final destination, and ectopically stop migrating in deep cortical levels (Caffrey et al., 2014; Chai et al., 2015; Gupta et al., 2002; Tabata and Nakajima, 2002). But interestingly, late-born neurons do reach upper cortical levels. It mainly seems to happen, however, in rostral cortices. 
A E17.5
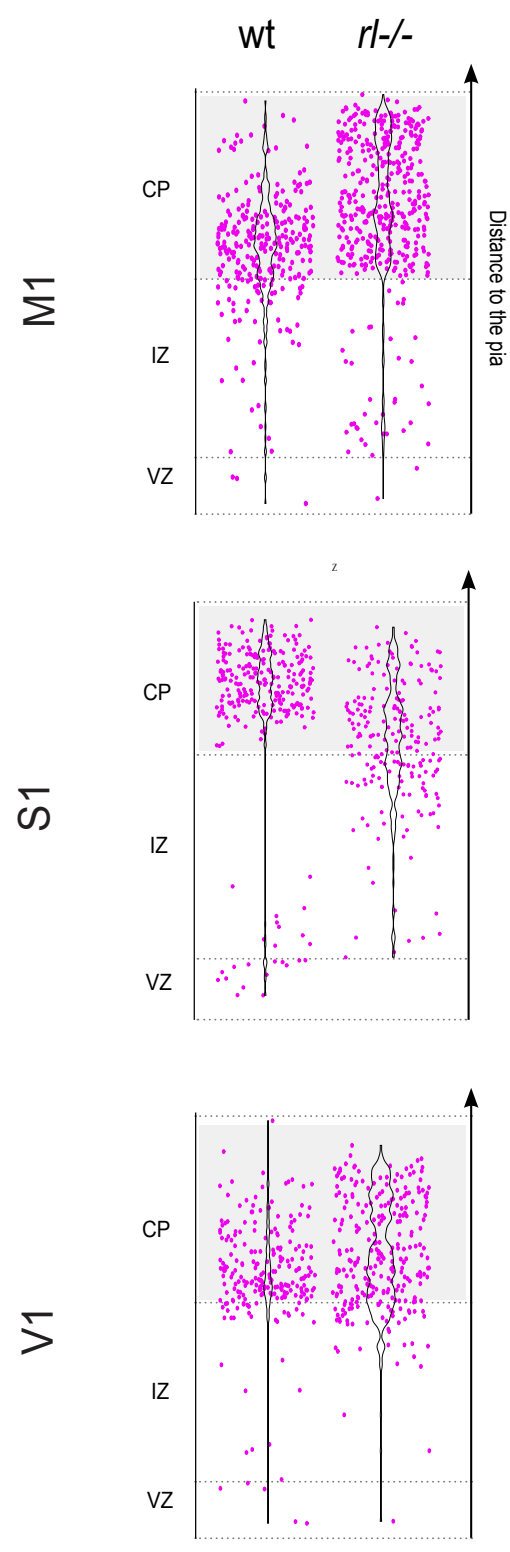

$\mathrm{B}$

P0
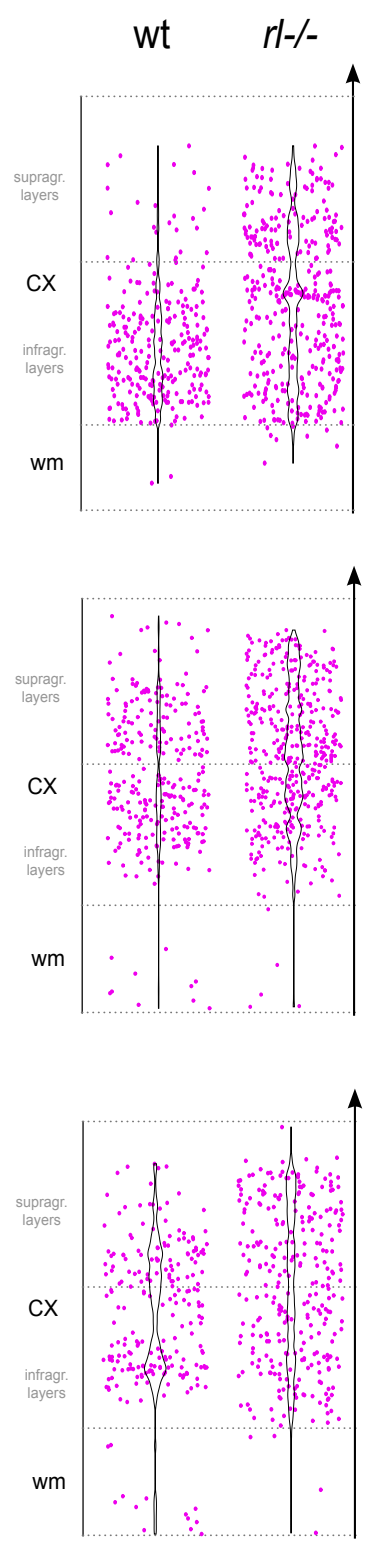

$\begin{array}{ll}\text { C } & \text { P7 }\end{array}$
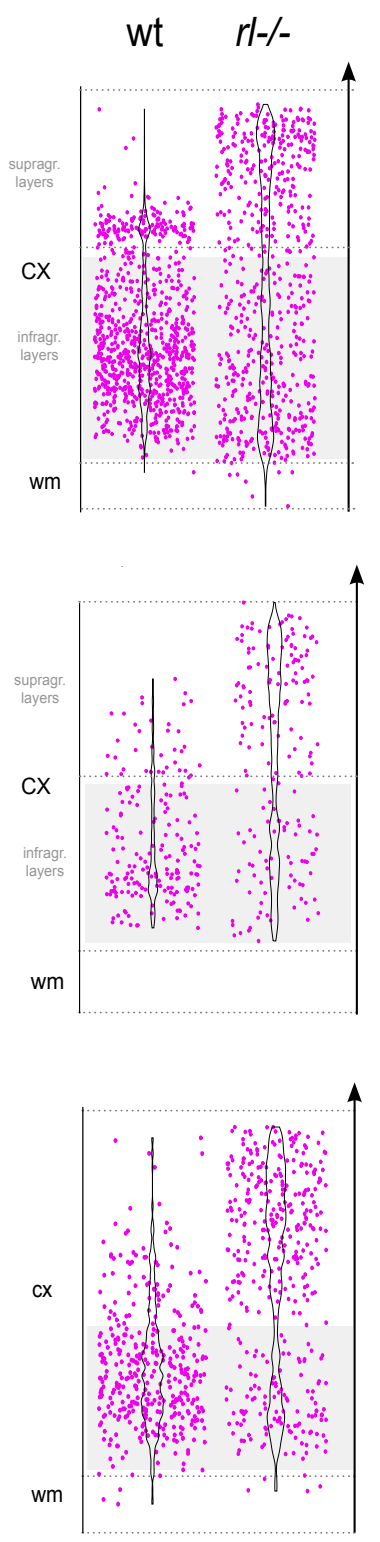

Figure 16. Distribution of E13.5-born neurons during development in the $\mathrm{M} 1, \mathrm{~S} 1$ and $\mathrm{V} 1$ of wild type and reeler cortices. Representative analysis of M1, S1 and V1 cortical columns from E17.5 (A), P0 (B) and P7 (C) coronal sections of EdU-injected (at E13.5) wt and rl-/- mice. The location of $\mathrm{EdU}^{+}$cells is circumscribed to lower layers in wild type. Reeler cortex transitions from a collective substantial cell dispersion to a differential areal distribution when migration is completed. Cortical plate (E17.5) and infragranular layers (P7) are shaded. Abbreviations: $c p$, cortical plate; iz, intermediate zone; vz, ventricular zone; cx, cortex. 

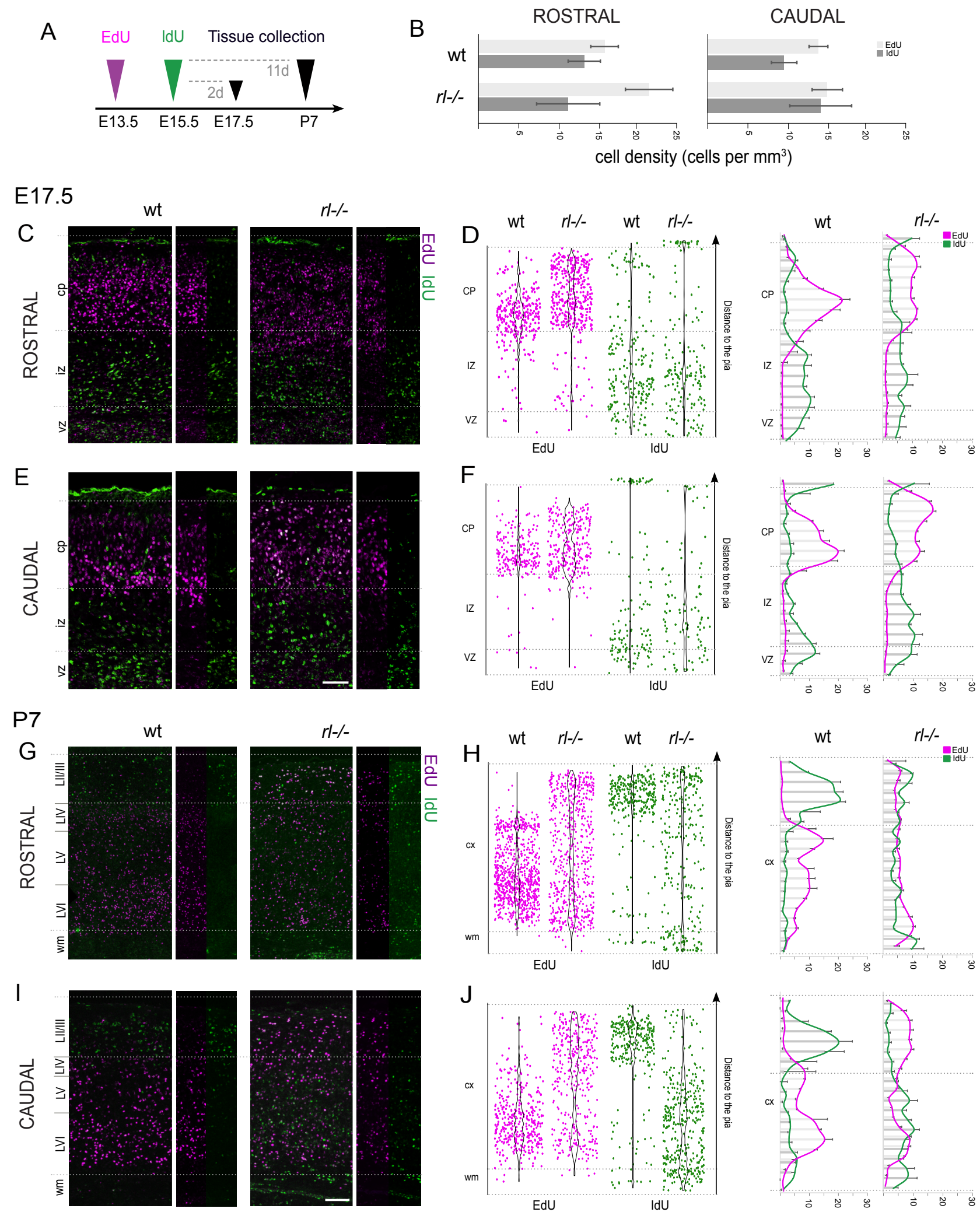
Figure 17. Development of opposing laminated patters in M1 and V1 in reeler. Experimental design (A). Cell density of E13.5- $\left(\mathrm{EdU}^{+}\right)$and $\mathrm{E} 15.5-\left(\mathrm{IdU}^{+}\right)$- born cells (50\%-brightest) in rostral and caudal cortices of wt and $r /$ /- mice (n=3-6) (1x1000) (B). Representative coronal sections immunostained for EdU and IdU of E17.5 and P7 injected wt and $r$-/- mice. Early- (EdU, magenta) and late- (IdU, green) born neurons in wt and mutant conditions are compared in the single-channel images (C, E, G, I). Cell distribution of the $50 \%$-brightest population of $C, E$, $\mathrm{G}$ and $\mathrm{I}$, shown as violin plots and cell density represented as percentage of cells per $\mathrm{mm}^{3}$ in a (20) binned cortical column $(D, F, H, J)$. Cells that share birth date migrate corresponding to the sequence they are born, as E13.5born cells reside superficially and E15.5-born cells are found bellow, in anterior and posterior regions in both genotypes. However, at P7 M1 and V1 show contrasting phenotypes in reeler, mirroring the adult laminated patterns. Abbreviations: $c p$, cortical plate; iz, intermediate zone; vz, ventricular zone; wm, white matter. Scale bars: $50 \mu \mathrm{m}(\mathrm{C}, \mathrm{E})$ and $100 \mu \mathrm{m}(\mathrm{G}, \mathrm{I})$.
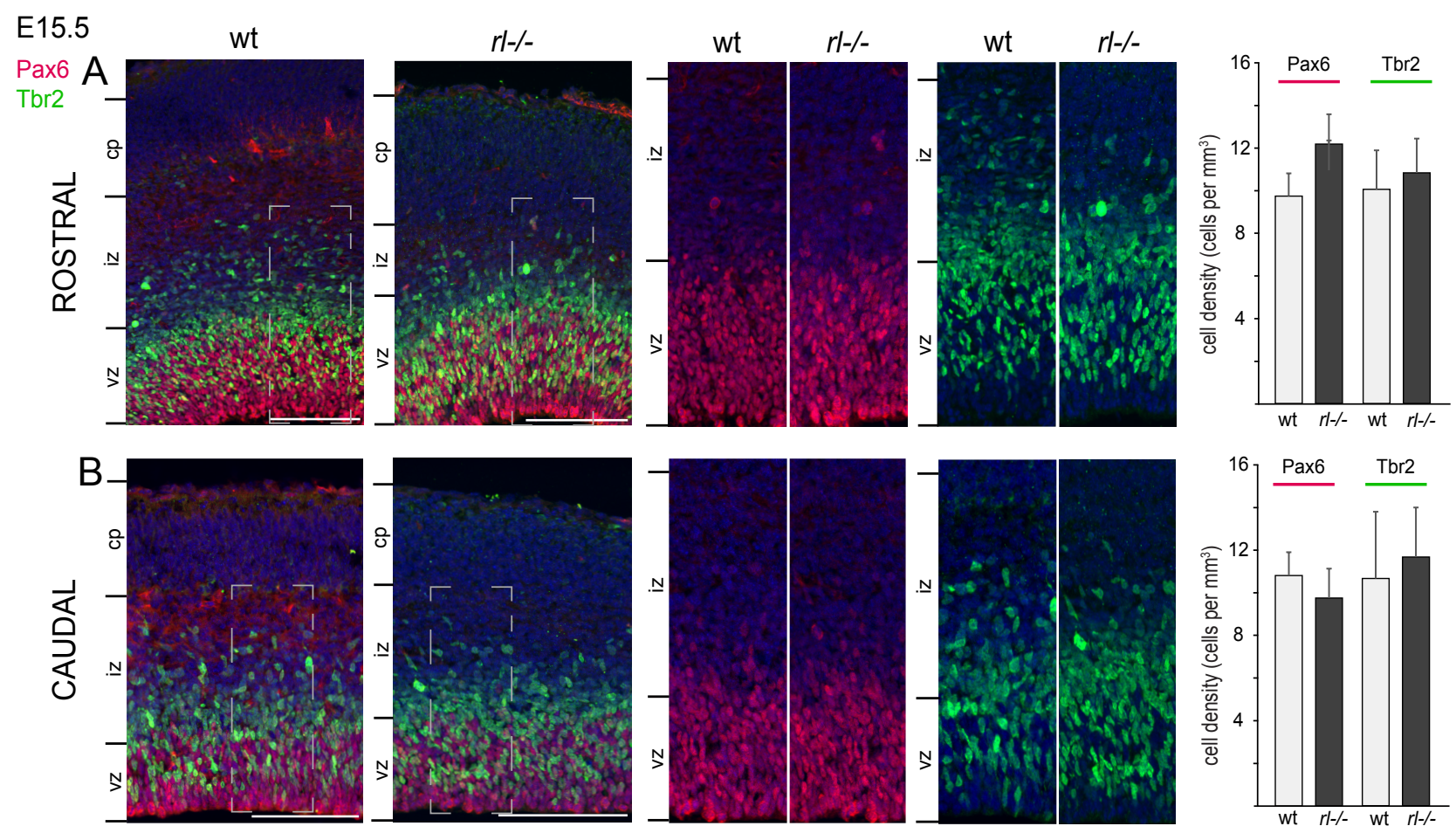

Figure 18. The progenitor pool is preserved in reeler. Immunohistochemistry for Pax6 (RGCs) and Tbr2 (IPs) in rostral (A) and caudal (B) cortices of wild type and Reelin-deficient E15.5 embryos. Bar charts represent cell density (cells per $\mathrm{mm}^{3}, 1 \times 1000000$ ) in $\mathrm{n}=3$. There are no substantial differences, neither in the location nor in the number of $\mathrm{Pax6}^{+}$(RGC in the VZ) or $\mathrm{Tbr}^{+}$(IPs in the SVZ) in anterior or posterior regions between the two genotypes. Abbreviations: $c p$, cortical plate; iz, intermediate zone; vz, ventricular zone. Scale bars: $100 \mu \mathrm{m}$. 


\section{TRACING OF TIME-LOCKED COHORTS OF NEWBORN NEURONS}

The Reelin-deficient neocortex did not show any major significant differences in the number of cells fated to a certain layer, cortical thickness, progenitor pool or cell density of the first progeny of early- and late- progenitors; neither in rostral or caudal regions in reeler, nor when compared to the wild type counterparts. Early- and late- born neurons migrate according to the sequence they are born in. Additionally, when corticogenesis is analyzed at late embryonic stages, early-born neurons are already in the cortex while late-born neurons are still located in the intermediate zone, as in the wild type developing cortex. However, the story seems to dramatically change afterwards, as P7 rostral and caudal cortices do not mirror the wild type lamination and show very distinct cell distributions. Some important questions emerged. For instance, what happens once cells enter the cortical plate? And, since neurons are known to be susceptible to Reelin right after they are born (Hirota et al., 2014; Jossin and Cooper, 2011; Trommsdorff et al., 1999; Uchida et al., 2009), how do different cohorts of projection neurons migrate below the cortical plate in a Reelin-deficient environment?

The detection by immunostaining of the halogenated thymidine analogues (with the exception of EdU) requires DNA denaturation, which might cause a slight disruption of the tissue. Thymidine analogues label, to a different extend, the progeny of a given progenitor and, thus, their application does not allow the discrimination of cells born by direct and indirect neurogenesis. Moreover, BrdU and BrdU-derivatives label the progeny of RGCs in the VZ but also IPs in the SVZ, obstructing a clear definition of the cells that have uptaken the labeling reagent. BrdU (and BrdUderivatives) birth dating analysis was the method of choice for studies that revolutionized the field and is still widely in use in proliferation and migration analyses. However, there are new techniques that ensure outstanding and highly reliable cell tracing, more suitable to our goal. One of them is the Flash Tag (FT) technique (Telley et al., 2016). This technique, based on the CSFE dye, fluorescently labels isochronic crowds of only VZ-derived cells. As it labels time-locked cohorts of migrating neurons, guarantees a fine-grained analysis. It also allows the discrimination of cells born by direct neurogenesis and a pure classification of the progenitor pool that uptake the dye. Moreover, CFSE- or FT- labeled cells are easily tracked during development as they can be simply detected by using the appropriate excitation wave length by fluorescence microscopy.

CSFE or FT labelling principle: The dye, carboxyfluorescein diacetate succinimidyl ester (CFDA), diffuses into the cells by crossing the plasma membrane. Once in the cytoplasm, CFDA is 
converted to CFSE, which is fluorescent and non-membrane permeable. CFSE then covalently binds to intracellular proteins ensuring a long-term fluorescence labeling (Quah and Parish, 2010). The dye can be visualized by using standard fluorescein filters (excitation and emission peaks at $492 \mathrm{~nm}$ and $517 \mathrm{~nm}$, respectively) by fluorescence microscopy. Signal can be also amplified with a rabbit anti-Fluorescein antibody.

In order to investigate in detail the migration kinetics and cell positioning in the IZ and in the CP of specific clusters of migrating neurons, the CFSE dye was injected into the lateral ventricles of E13.5 or E15.5 embryos. Early- and late- born labeled neurons were then analyzed at crucial points of embryonic and postnatal development. All pups were subjected to injection as wild type, heterozygous and reeler genotypes are indistinguishable in the uterine horns. If applicable, osmotic pumps for chronic BrdU labeling or single i.p. EdU injections were done immediately after surgery.

\section{Defining the first progeny of FT cells}

CSFE is rapidly diluted and for a presumably known number of cell cycles, as it is diluted to a half in each mitosis. Halogenated thymidine analogs incorporate into the S-phase of diving cells, while CSFE diffuses to the juxtaventricular cells. According to the distribution of the progenitor pool and the principles of the interkinetic nuclear migration, BrdU is then integrated into apical-SVZ and basal-VZ cells in S-phase, whereas CSFE (from now on referred only as Flash-Tag or FT) is incorporated by apical-VZ cells in M-phase (Fig.19 A).

To visualize cells that continued dividing, a simultaneous FT-pulse labeling and a chronic BrdU administration was conducted on E13.5 and E15.5 pups and analyzed four days later (Fig.19 B-F). The continuous BrdU administration after the FT injection showed that most of the top 50\%brightest FT-cells in wild type animals are born from progenitors in the $\mathrm{VZ}$, as $>85 \%$ of the $\mathrm{FT}^{+}$ were $\mathrm{BrdU}^{-}$(85.45\% \pm 12.64). On the contrary, in reeler animals, although still in majority, just around $60 \%$ of $\mathrm{FT}^{+}$remained $\mathrm{BrdU}^{-}(60.75 \% \pm 4.47)$. When applying further thresholding, $>97 \%$ of $\mathrm{FT}^{+}$were $\mathrm{BrdU}^{-}$in the 30\%- brightest fluorescence group (wt: 97.89\% 0.77, rl-/-: 95.24\% 0.90) and close to $99 \% \mathrm{FT}^{+}$were $\mathrm{BrdU}^{-}$for the $20 \%$ - brightest fluorescence cluster ( $w t$ : $99.09 \% \pm 0.90, r$ /-: $98.96 \% \pm 0.08$ ). Thresholding, aiming for the top $20 \%$-brightest fluorescence group, was therefore applied in upcoming analysis, as the population of interest was the FT+ newborn cells which were not involved in sequential mitosis. 

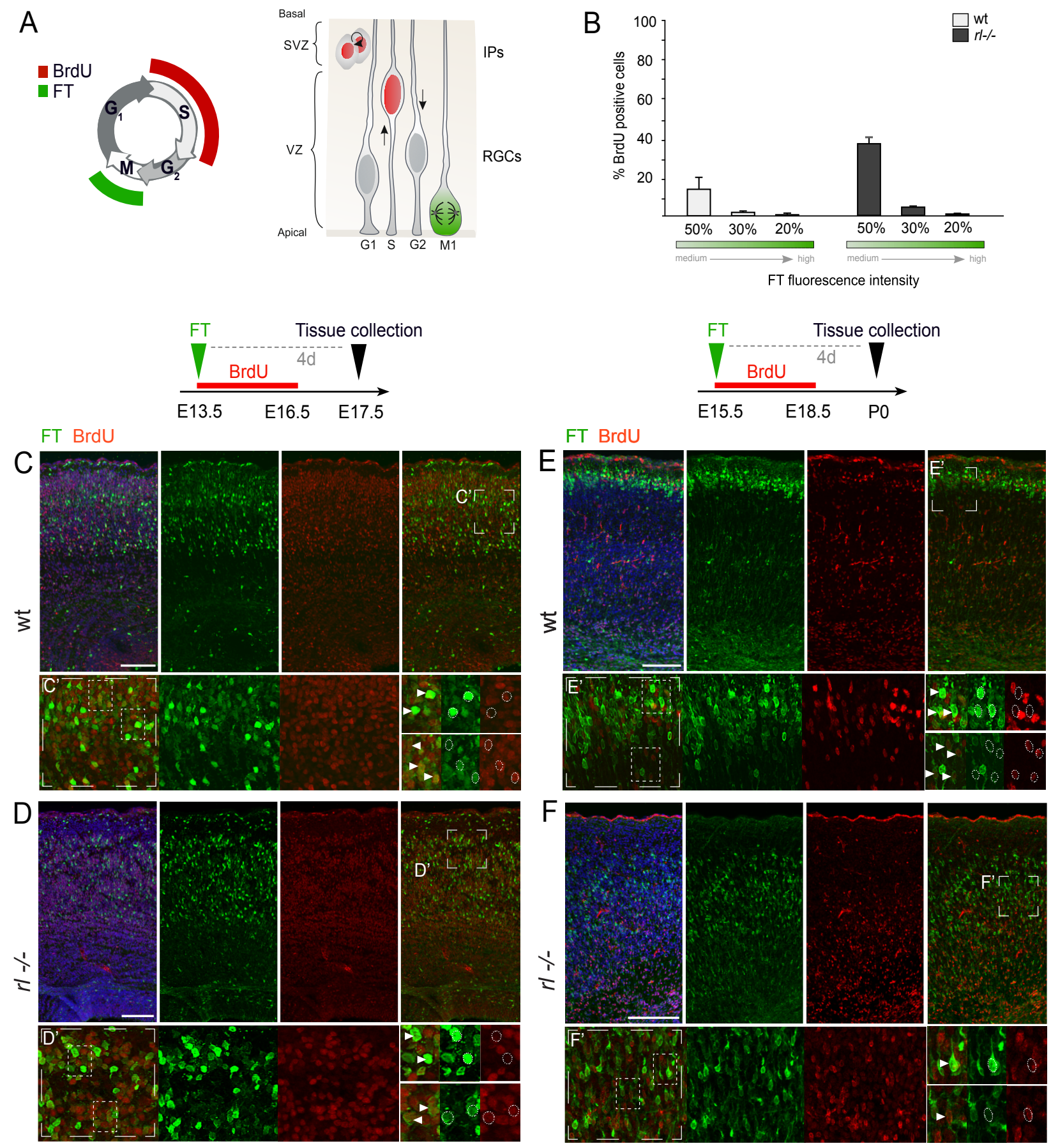

Figure 19. Labeling of apical-VZ- and SVZ/ basal-VZ - derived progeny by Flash Tag and BrdU. Illustration of FlashTag (FT) and BrdU labeling principle (A). Quantification of BrdU and FT co-labeling after thresholding (50\%, 30\%- and 20\%- brightest FT population) in wt and rl-/- (B). Immunohistochemistry for FT and BrdU on E17.5 $(C, D)$ and $P O(E, F)$ wt and $r l-/$ - coronal sections, four days after the FT injection. Zoom-ins in $C^{\prime}, D^{\prime}, E^{\prime}$ and $F^{\prime}$ show $\mathrm{FT}^{+} / \mathrm{BrdU}^{-}$and $\mathrm{FT}^{+} / \mathrm{BrdU}^{+}$cells (arrowheads and dotted circles) in both genotypes. Flash-tagged cells are mainly born from VZ-progenitors, as they largely remain BrdU negative. The 20\%-brightest FT population does not show immunoreactivity for BrdU, neither in wt nor in rl-/-. Abbreviations: SVZ, subventricular zone; IZ, intermediate zone; VZ, ventricular zone; IPs, intermediate progenitors; RGCs: radial glia cells. Scale bars: $100 \mu \mathrm{m}$. 


\section{Tracing of early-born neurons}

It has been shown that migrating cells express the main Reelin receptors (especially ApoER2) already in the subventricular zone (Hirota et al., 2014), and that most likely they bind to cleaved Reelin fragments that diffuse from the marginal zone (Jossin et al., 2007). Thus, Reelin has presumably an effect on neurons that are still on their way to the cortical plate. Pulse labeling with EdU showed that early-born (E13.5-) neurons reside in the cortical plate four days after the injection in Reelin-deficient mice, in general mirroring the wild type littermates. However a finegrained visualization of those cells at early stages of migration and after reaching the cortical plate remain elusive. An additional motivation to conduct these experiments was that little is known about the migration of LVI cells and the influence of Reelin on these neurons. Aiming to answer those questions, FT pulses were performed on E13.5 pups and analyzed two and four days later. Representative images (native signal) and violin plots are shown in Figure 20. The quality of the native signal can be judged in the insets, where even some morphological details of migrating neurons (e.g. leading processes) can be distinguished - not shown previously (Telley et al., 2016).

Two days post-injection (E15.5), the overviews from coronal sections (Fig.20 A-D) illustrate a migration wave, in a rostro-caudal and medio-lateral gradient. The comparison of $\mathrm{FT}^{+}$ cells in a lateral-to-medial range gives such a developmental time difference that allows the examination of migrating cells before and after they enter the cortical plate. In lateral regions, despite minor differences in the caudal cortex, FT cells are restricted to the uppermost sector of the IZ in both genotypes (Fig.20 A"- D" and H). In medial zones, cells in wild type shape a layer beneath the pia mater (Fig.20 A', $\mathrm{C}^{\prime}$ and H). However, in the Reelin-deficient neocortex $\mathrm{FT}^{+}$cells are still in the mid- (rostral) or lower- (caudal) part of the cortical plate (Fig.20 B', D' and H).

Four days post-injection (E17.5), all FT cells have reached the CP in wild type and reeler mice (Fig.20 E, F and I). No layering is obvious in reeler. However, in the rostral reeler cortex $\mathrm{FT}^{+}$cells appear to concentrate into an ascending gradient (from lower to upper levels of the cortical plate) more equivalent to the wild type littermates (Fig. $20 \mathrm{E} \mathrm{rl-/-,} \mathrm{arrowheads),} \mathrm{while} \mathrm{the} \mathrm{inversion} \mathrm{starts}$

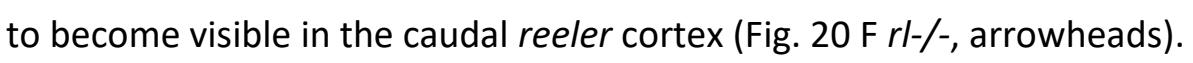



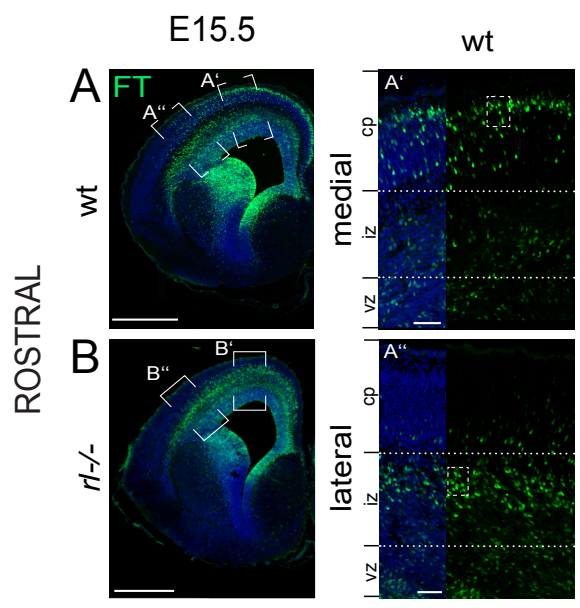

$r l-/-$

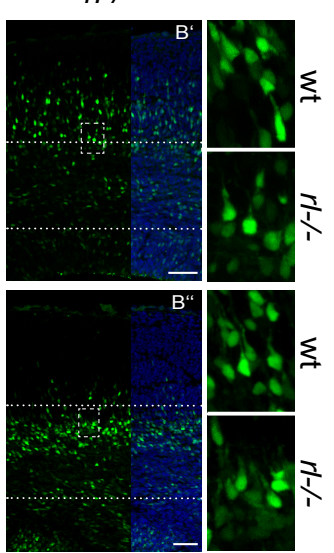

wt
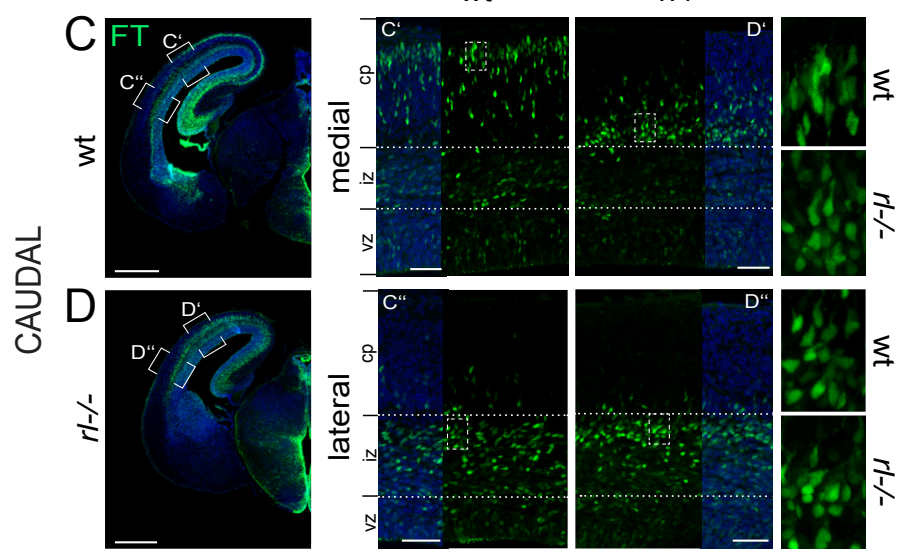

E17.5

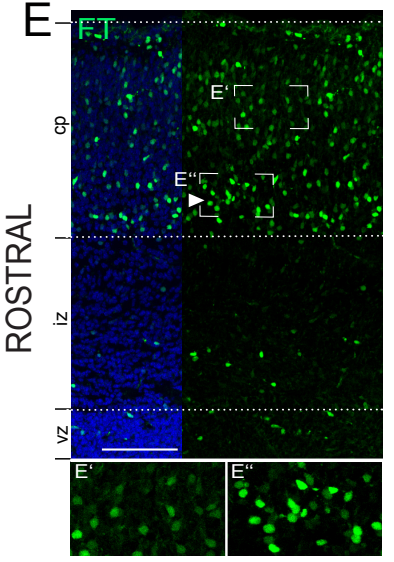

wt

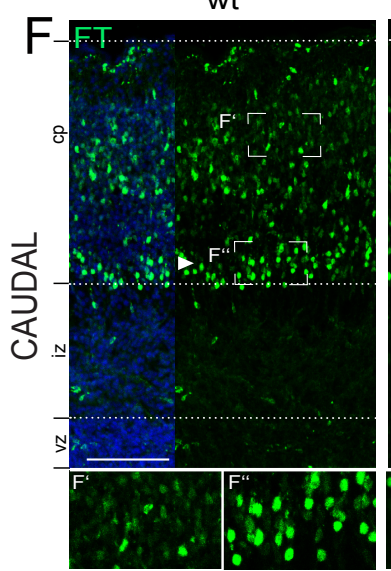

$r l-/-$

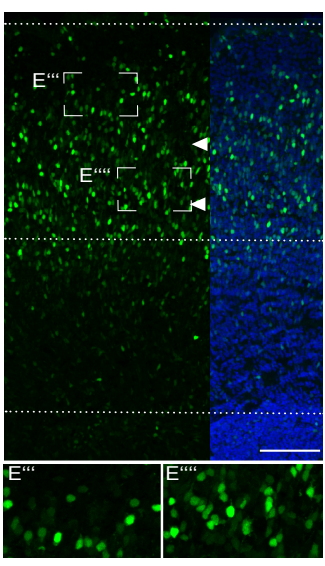

$r l-/-$

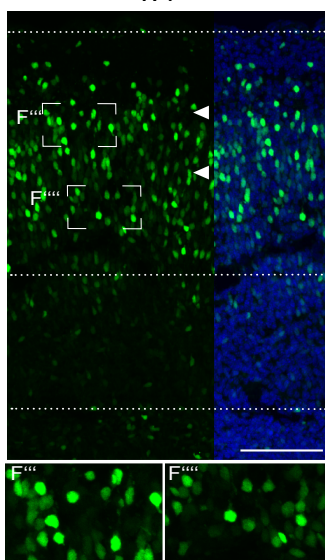

G

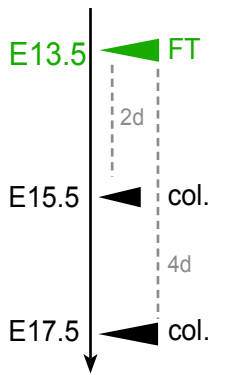

H ROSTRAL

E15.5

CAUDAL

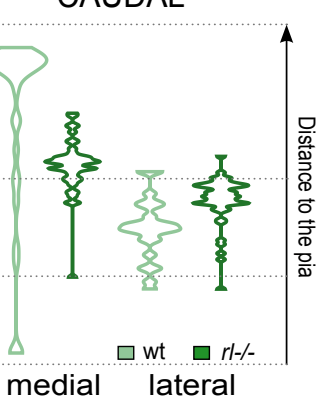

I ROSTRAL CAUDAL

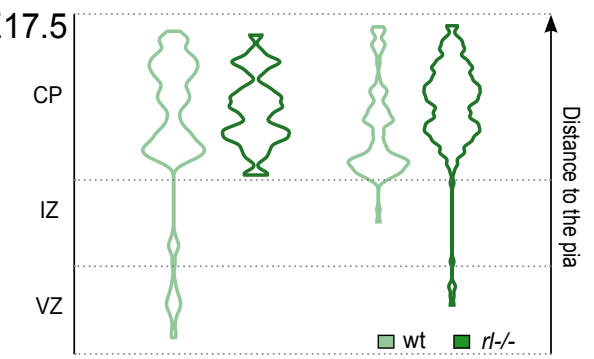

Figure 20. Tracing of early-born FT-labeled neurons. Immunohistochemistry for FT on wt and $r$ l-/- coronal sections, two days (E15.5, A-D) and four days (E17.5, E,F) after the FT injection (E13.5). Experimental principle (G). Lengthwise distribution of FT cells in cortices shown in A-D (H) and E-F (I) shown as violin plots. $\mathrm{FT}^{+}$cells migrate in a cohesive population prior entering the cortical plate in wt and $r /$-/.. $\mathrm{FT}^{+}$labeled cells are clearly delayed while migrating through the cortical plate and show a great dispersion in reeler. The rostral cortex show a more comparable distribution to wild type, while in the caudal cortex there are already traces of the inverted pattern (arrowheads). Abbreviations: $c p$, cortical plate; iz, intermediate zone; $v z$, ventricular zone. Scale bars: $500 \mu \mathrm{m}$ (overviews, A-D), $50 \mu \mathrm{m}\left(\mathrm{A}^{\prime}-\mathrm{D}^{\prime}, \mathrm{A}^{\prime \prime}-\mathrm{D}^{\prime \prime}\right)$ and $100 \mu \mathrm{m}(\mathrm{E}-\mathrm{F})$. 


\section{Tracing of late-born neurons}

Layer II/III cells showed opposite cell distribution in M1 (rostral) and V1 (caudal) of the adult Reelin-deficient neocortex. The cell birth dating analysis with IdU (injected at E15.5) indicates that there is a dispersed location of E15.5-born neurons below the cortical plate in the M1 and V1 cortex of E17.5 wild type and reeler embryos, while those cells are mainly located in upper- (rostral) or lower- (caudal) cortical levels at P7. However, when during corticogenesis reeler deviates from an equivalent normal development and when the discrepancies in rostral and caudal regions start in the mutant mouse, remains unresolved. Moreover, early-born neurons, but also late-born neurons are supposed to respond to Reelin below the cortical plate. Thus, will they migrate as a compact layer in the absence of Reelin as well? In order to precisely trace layer II/III cells and answer those questions, FT pulses were done on E15.5 pups and analyzed two (E17.5) and four (PO) days later, as for the tracing of early-born neurons. Representative images and violin plots are shown in Figure 21 . The quality of the native signal can be judged in the insets, where the typical morphology of migrating projection neurons can be identified.

Two days post-injection (E17.5), the overviews from coronal sections (Fig.21 A-D) provide a general idea of the distinct location of the FT cells medio-laterally. In lateral regions, where cells still reside in the $\mathbf{I}$, no major differences, apart from a marginal delay in reeler, were found between the genotypes (Fig.21 A"- D" and H). Thus, the first generation of late-born $\mathrm{FT}^{+}$cells migrates in a coherent population along the IZ, comparable to the FT labeled early-born neurons, with just few cells left behind (in ventricular zone) or found above (in the marginal zone) (Fig.21 $\mathrm{H})$. The morphology of the migrating neurons in the marginal zone indicates that those cells might correspond to interneurons (data not shown). On the contrary, in rostro-medial provinces, where cells have already started to bypass previously born cortical neurons in wild type, the location of $\mathrm{FT}^{+}$cells is still restricted to the $\mathrm{IZ}$ in reeler (Fig.20 $\mathrm{A}^{\prime}$ and $\mathrm{B}^{\prime}$ ). $\mathrm{FT}$ labeled cells are also constrained to the intersection between the $\mathrm{CP}$ and the $\mathrm{IZ}$ in caudal wild type and reeler, corresponding to the progression of the migration wave (Fig.21 $C^{\prime}$ and $D^{\prime}$ ).

Four days later (PO) (Fig. $21 \mathrm{E}, \mathrm{F}, \mathrm{I}$ ), supragranular cells have bypassed previously born neurons in wild type, and constitute the future layer II/III on top of the cortical plate (Fig.21 E,F,I wt). However, migration is, to a different extent, altered in the absence of Reelin. Aside from an obvious cell scattering, $\mathrm{FT}^{+}$cells seem to have travelled further distances in the rostral cortex (Fig.21 E rl-/-, arrowheads), in comparison to the caudal cortex, where these neurons are restricted to the lower-layer zone (Fig.21 F rl-/-, arrowheads). 

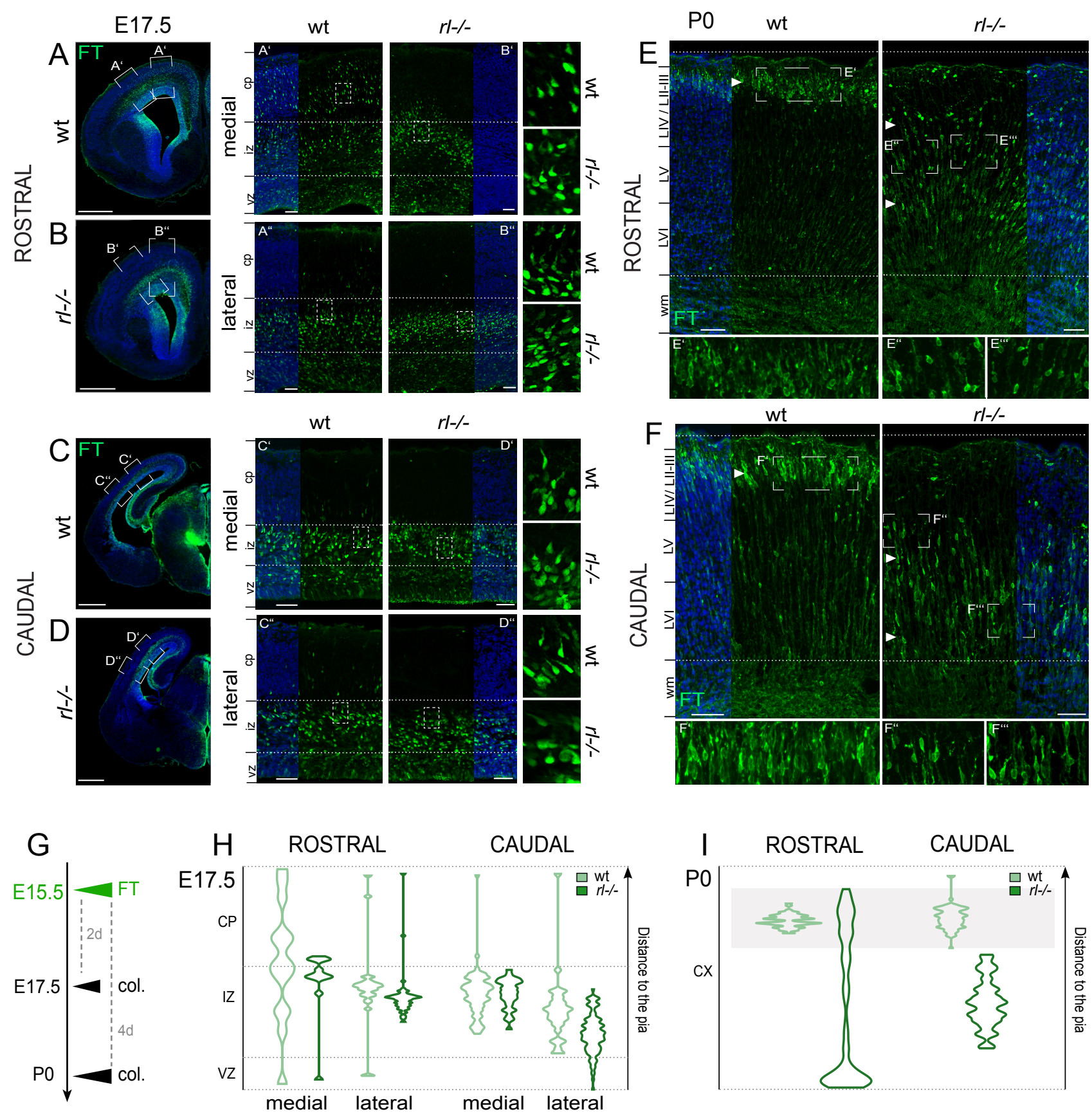

Figure 21. Tracing of late-born FT-labeled neurons. Immunohistochemistry for FT on wt and $r l$-/- coronal sections, two days (E17.5, A-D) and four days (PO, E,F) after the FT injection (E15.5). Experimental principle (G). Radial distribution of FT cells in a cortical column of A- F shown as individual violin plots $(\mathrm{H}, \mathrm{I})$. Layer II/III is shaded in $\mathrm{I}_{\text {. }} \mathrm{FT}^{+}$cells migrate in a cohesive population as they navigate through the intermediate zone in wt and rl-/-. $\mathrm{FT}^{+}$labeled cells are clearly delayed while migrating through the cortex, especially in the caudal cortex in reeler, where all cells are located below the equivalent to LII/III in wt (arrowheads). Abbreviations: $\mathrm{cp}$, cortical plate; iz, intermediate zone; vz, ventricular zone; wm, white matter. Scale bars: $500 \mu \mathrm{m}$ (overviews, A-D), $50 \mu \mathrm{m}$ $\left(A^{\prime}-D^{\prime}, A^{\prime \prime}-D^{\prime \prime}\right)$ and $100 \mu m(E-F)$. 


\section{Neuronal positioning and cell fate of layer II/III cells postnatally}

We have shown that neurons migrate as a cohesive population as they cross the intermediate zone in a Reelin-deficient environment, regardless of the time they are born. Based on the cortical distribution of E15.5- FT labeled cells in newborns, rostral and caudal phenotypes are also likely to be shaped by the time when neurons migrate through the cortical plate. Layer II/III cells are especially interesting because, as they migrate the last, they might also influence the final location of their predecessors. Moreover, they share the pattern of migration with layer $\mathrm{V}$ and layer IV cells, as they all migrate first by glia-guided locomotion and later by somal translocation.

\section{Cortical location of VZ- and VZ/SVZ- derived progeny}

Excitatory neurons arise from RGCs in the VZ and IPs in the SVZ. We have learned from the FT-cell tracing (VZ-derived) that there is a large difference in the relative location of $\mathrm{FT}^{+}$neurons in wild type and reeler newborns, also when comparing rostral and caudal sections of the mutant mouse. However, we are lacking information about the severity of the migration delay. Additionally, the migration kinetics of basal-VZ and SVZ-derived layer II/III cells is still a question.

The progeny of the different progenitor pools was tagged by a simultaneous FT- (VZ- or apical progenitors) and EdU- (SVZ- or basal progenitors) pulse at E15.5 and analyzed four days later (Fig.22). Thresholding to discriminate the first generation of post-mitotic cells after the pulses was applied as described for previous experiments. In wild type, E15.5-FT labeled cells (shown in green) constitute a layer beneath the pial surface. More than $95 \%$ of $\mathrm{FT}^{+}$cells, in rostral and caudal sections, are found from bins 1 to 8 ( $\left.w t_{\text {rostral }}: 95.58 \% \pm 3.15 ; w t_{\text {caudal }}: 95.27 \% \pm 5.81\right)$. Although showing a greater scattering, the progeny of the basal-RGC and IP progenitors, shown as E15.5-EdU labeled cells (in magenta), is also mainly restricted to the same location ( $\mathrm{wt}_{\text {rostral: }}$ 77.05\% \pm 14.03 ; wt $_{\text {caudal: }}$ 70.17\% \pm 11.58 ) (Fig.22 B, E and H). In the absence of Reelin, E15.5-FT ${ }^{+}$cells fail to constitute a layer and show a strong cell dispersion. However, more than $50 \%$ of $\mathrm{FT}^{+}$cells were found in the equivalent to layer II/III in wild type in rostral cortices, while just less than $5 \%$ of those cells have reached bins 1-8 in caudal sections in reeler ( $r /-/$-rostral: $52.46 \% \pm 13.9 ; r l-/-$ caudal: $4.41 \% \pm 6.31$ ) (grey shade in Fig. $20 \mathrm{H}$ ). It can be therefore confirmed that the cohort of E15.5-FT+ cells in rostral sections has migrated further distances, in comparison to caudal cortices $(p=0.003)$, under an equivalent Reelin-deficient environment. The E15.5- EdU labeled population shows a comparable cell distribution silhouette, as most of the $\mathrm{EdU}^{+}$cells are found at lower cortical levels (bins 9-20 = rl-/-rostral: 79.91\% $\pm 10.98 ;$ rl-/-caudal: 80.75\% \pm 6.38 ) (Fig.22 C, F and H). Thus, the distinct 
rostral and caudal phenotypes seem to depend on both, direct and indirect neurogenesis for RGCS and IPs.

\section{Where are you when migration ends?}

Cell tracing with Flash Tag has shown that migration of late-born neurons is more advanced in rostral cortices at P0 in reeler. However, migration at P0 is not over yet. Relative cell location of late-born neurons was analyzed on E15.5 FT- injected mice at P7, when neuronal migration is known to be completed. Representative images and violin plots are shown in Figure 23. In wild type, E15.5- $\mathrm{FT}^{+}$cells have settled down in the LII/III zone (bins 3-7: $\mathrm{wt}_{\text {rostral }}$ : $100 \%$; $\mathrm{wt}_{\text {caudal }}$ : $98.30 \%$ ) (Fig.23 B, C, D wt). Most of the E15.5- FT labeled cells in reeler are dispersed within the zones corresponding to granular-to-infragranular layers in wild type (bins 8-20 = rl-/-rostral: 71.79\%; rl-/caudal: 93.33\%) (Fig.23 B, C, D rl-/-). However, those cells are still located more superficially in rostral cortices when compared to caudal sections (bins 3-7 = rl-/-rostral: $28.20 \% ; r l-/$-caudal: $6.66 \%$ ), indicating that late-born neuron do migrate further distances in rostral sections within equivalent periods of time in reeler.

Figure 22. Cell distribution of layer II/III VZ- and VZ/SVZ- derived in the premature cortex in PO wild type and reeler mice. Pups were subjected to FT and EdU injections at E15.5, in order to label VZ-derived and VZ/SVZsupragranular cells, respectively. Immunostaining for FT (in green) and EdU (in magenta) on PO rostral (A, B and C) and caudal (D, E and F) wt and $r /$-/- coronal sections, four days after the injections. Experimental principle and schematic representation of the FT and EdU labeling approach (G). Quantification of the relative cell distribution of $\mathrm{FT}^{+}$and $\mathrm{EdU}^{+}$cells in $\mathrm{M} 1$ and $\mathrm{V} 1$ wt and $\mathrm{rl}-/$ - (20 binned) cortical columns ( $\mathrm{n}=3$, in \% of cells per $\mathrm{mm}^{3}$ ). Thresholds (FT: 20\%-, EdU: 50\%- brightest) to sort the first progeny after the pulse were apply are described above $(\mathrm{H})$. $\mathrm{FT}^{+}$remains largely EdU in both genotypes ( $\left.w t: \mathrm{B}^{\prime}, \mathrm{B}^{\prime \prime}, \mathrm{E}^{\prime}, \mathrm{E}^{\prime \prime} ; r l-/-: \mathrm{C}^{\prime}, \mathrm{C}^{\prime \prime}, \mathrm{F}^{\prime}, \mathrm{F}^{\prime \prime}\right)$. EdU and FT labeling is restricted to the same cortical level. Layer II/III cells are capable to reach upper layers in rostral regions in reeler, in contrast to caudal cortices where those cells are still at lower cortical levels. Abbreviations: $\mathrm{cx}$, cortex; wm, white matter. Scale bars: $500 \mu \mathrm{m}(\mathrm{A}, \mathrm{D})$ and $50 \mu \mathrm{m}(\mathrm{B}, \mathrm{C}, \mathrm{E}, \mathrm{F})$. 
ROSTRAL
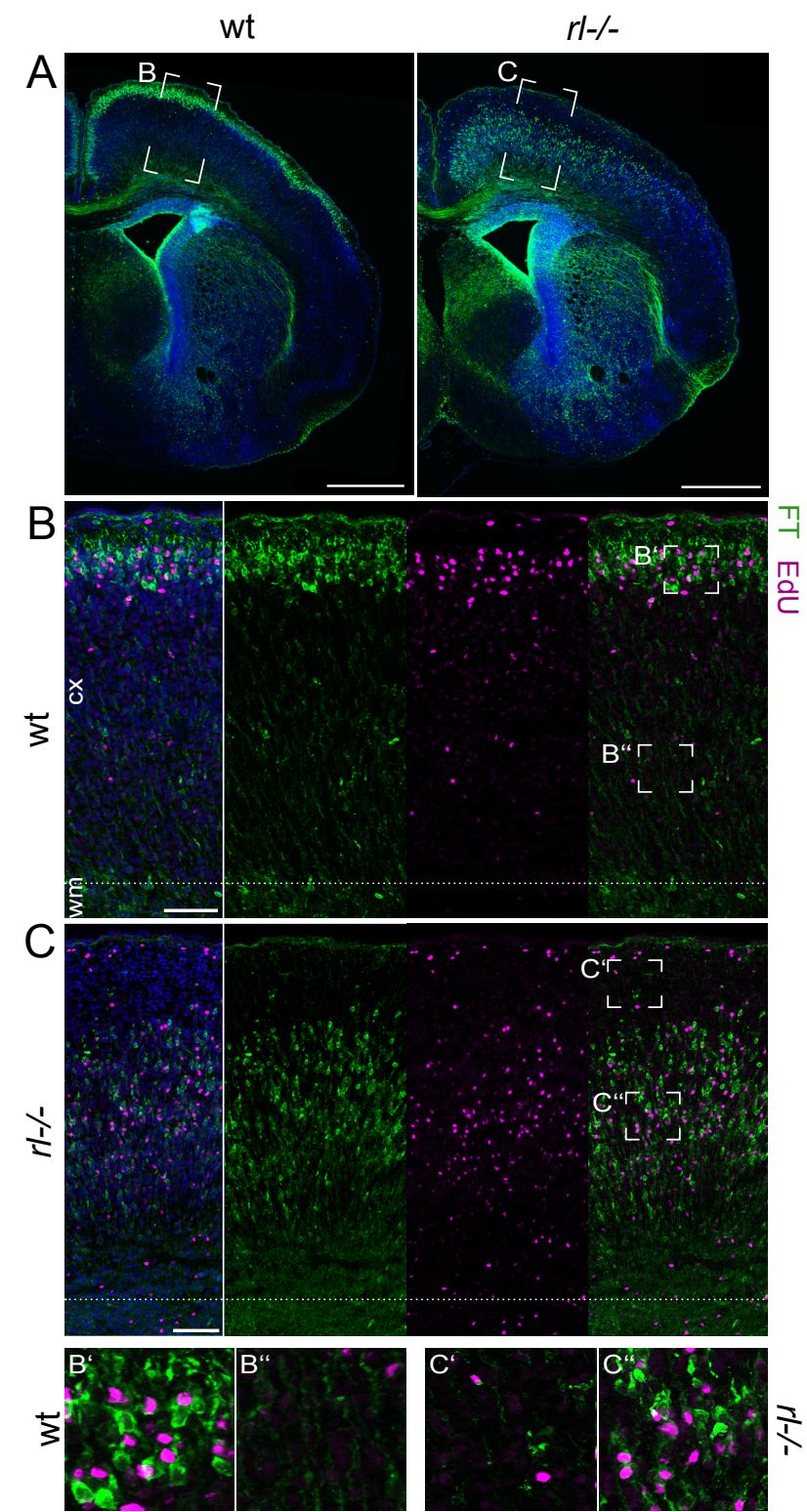

G
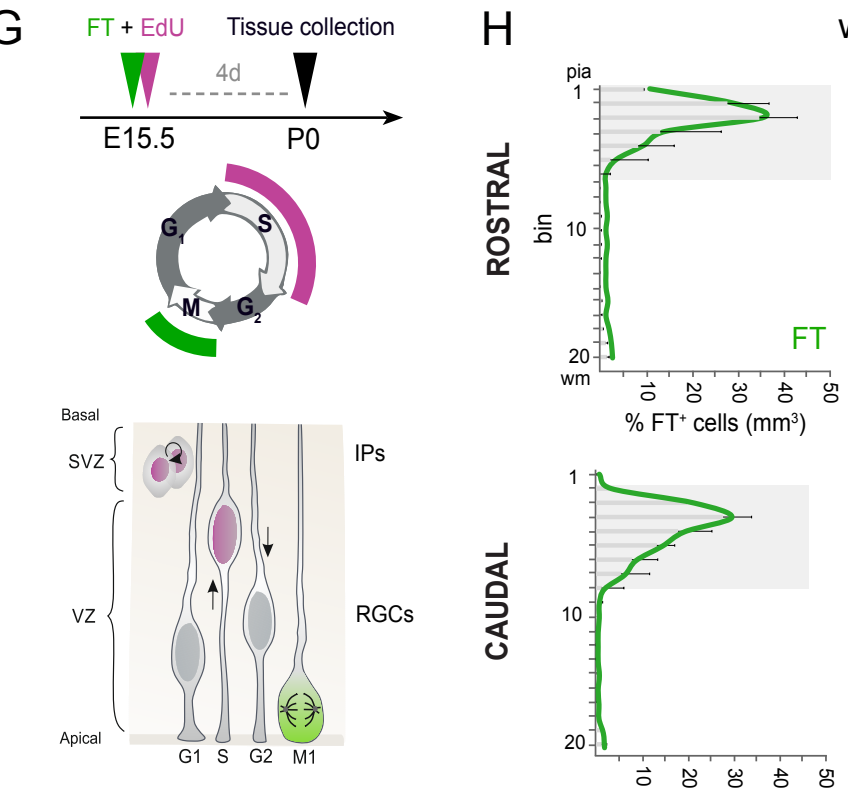

CAUDAL
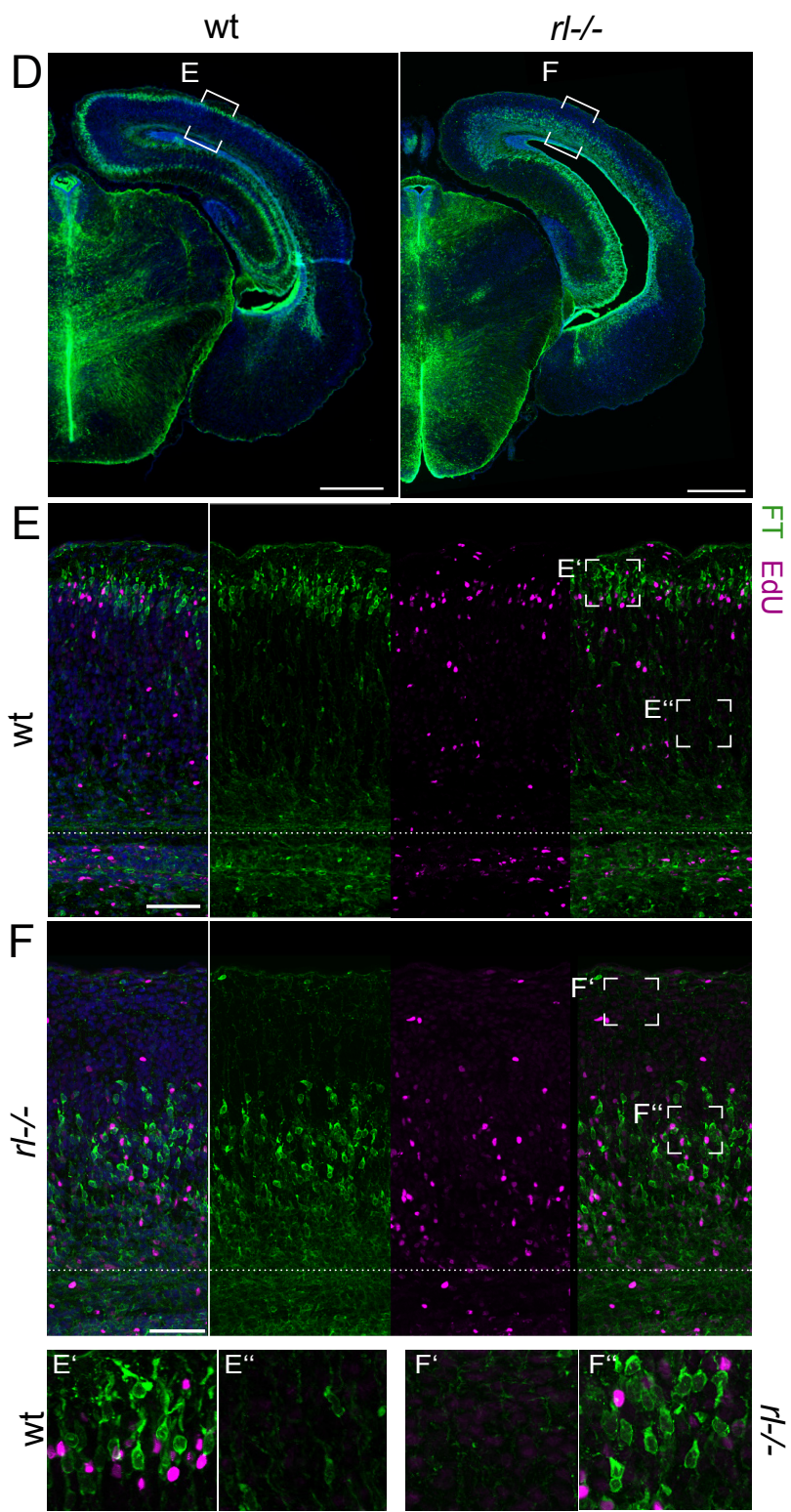

wt

rl--
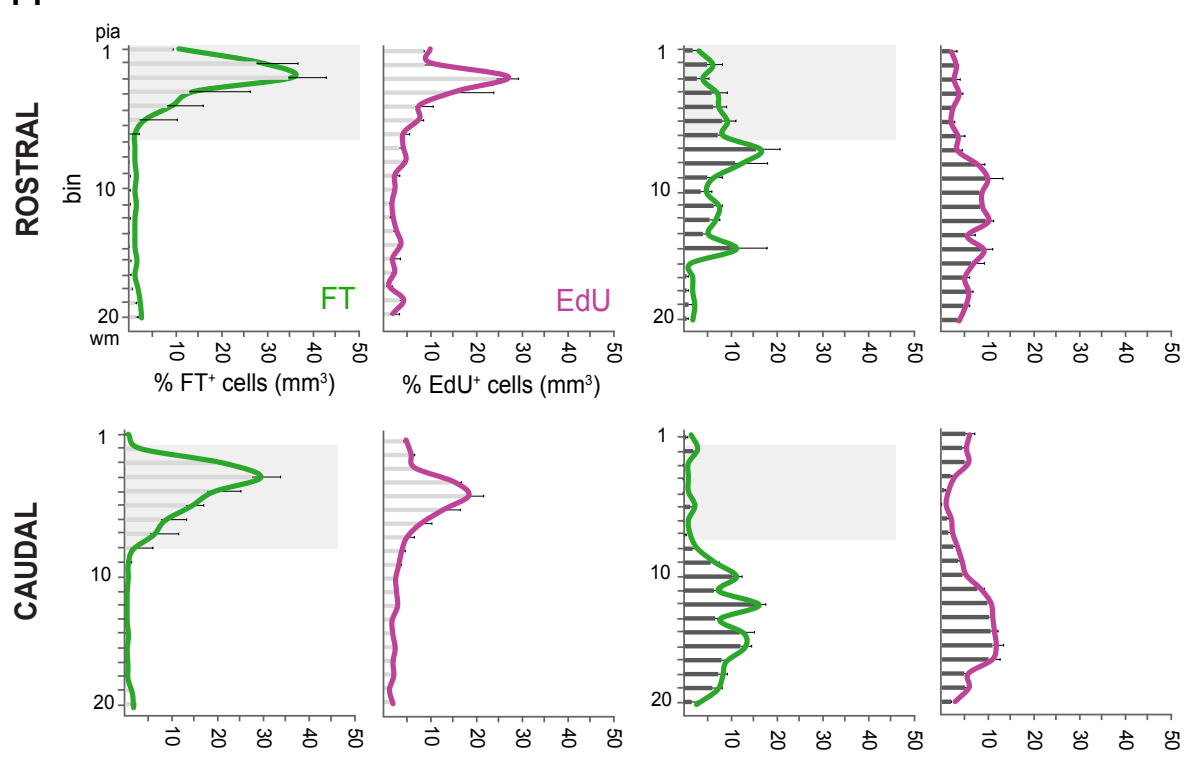

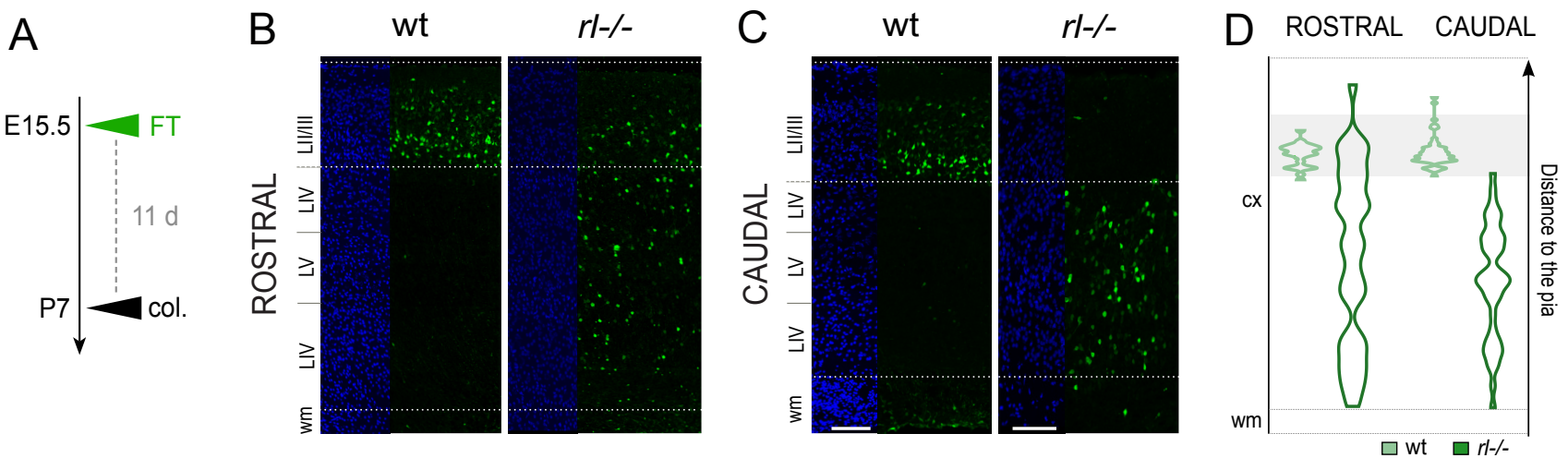

Figure 23. Final cortical location of layer II/III cells in wild type and reeler. Experimental principle (A). E15.5 embryos were subjected to a FT-injection, in order to label supragranular cells. Immunostaining for FT on rostral (B) and caudal (C) wt and rl-/- coronal cortices, eleven days post-injection. Lengthwise distribution of FT cells throughout the cortex shown as violin plots $(\mathrm{D}, \mathrm{n}=2)$. $\mathrm{FT}^{+}$cells reside in the LII/III zone in wild type. In reeler, cells in rostral and caudal sections show a pronounced cell scattering. $\mathrm{FT}^{+}$cells in the caudal reeler cortex are unable to reach upper layers. Abbreviations: cx, cortex; wm, white matter. Scale bars: $100 \mu \mathrm{m}$

\section{Cell fate of ectopically located late-born neurons}

There is a tight association between the birth date of a projection neuron and its layer- or cellfate (Chen et al., 2005; Franco et al., 2012; Leone et al., 2008; Nieto et al., 2004). Cell identity could be also determined by the intrinsic molecular phenotype of a given cell, regardless of their birthdate (Hevner et al., 2003a). What will be the cell identity if we face a neocortex with no layers, with cells ectopically located? In order to answer that question, cell fate was evaluated in late-born cells in the well-layered wild type and in the disorganized reeler cortex. Future layer II/III cells were labeled by FT at E15.5 and pups were harvested at P0 and P7. The fate of the FT+ cells was examined by immunostaining for Satb2, a marker for upper layer neurons (layer II/III and layer IV) (Britanova et al., 2008) and Ctip2, a marker for lower layer (mainly layer V) neurons (Arlotta et al., 2005) (Fig.24 and Fig.25)

At PO (4 days after the injection) (Fig.24), in anterior and posterior regions of the wild type cortex roughly half of the E15.5- $\mathrm{FT}^{+}$population were positive for Satb2 ( $\mathrm{wt}_{\text {rostral: }}$ 45.86\% \pm 7.24 ; $\left.w_{\text {caudal }}: 46.23 \% \pm 9.97\right)$, whereas less than $1 \%$ showed immunoreactivity for Ctip2 $\left(w_{\text {rostral: }} 0 \% \pm 0\right.$; $w t_{\text {caudal }}: 0.72 \% \pm 1.02$ ) (Fig.24 A, C and E wt). In the Reelin-deficient neocortex, E15.5- $\mathrm{FT}^{+}$cells do not show any preference for a certain cell fate, neither in rostral nor in caudal cortices, as co- 
labeling with the cell identity markers is less than $10 \%$ in both regions ( $r$ l-/-rostral: Satb2 ${ }^{+}=6.57 \% \pm 2.94$, Ctip2 $^{+}=7.58 \% \pm 0.28 ; \quad r l-/$-caudal: Satb2 $^{+}=7.38 \% \pm 3.60 ;$ Ctip2 $^{+}=3.60 \% \pm 0.44$ ) (Fig.24 B, D and E rl-/-) .

At P7 (11 days after the injection) (Fig.25), E15.5- $\mathrm{FT}^{+}$cells accumulate on upper cortical levels and constitute part of layer II/III in wild type. On average, $70 \%$ of those cells were Satb2 ${ }^{+}$ and about $100 \%$ remained Ctip2 ${ }^{-}\left(\right.$wt $_{\text {rostral }}$ : Satb2 $2^{+}=82.69 \% \pm 10.87$, Ctip2 $^{+}=0.29 \% \pm 0.41 ;$ wt $_{\text {caudal: }}$ : Satb2 $^{+}=64.63 \% \pm 12.30$, Ctip2 $\left.^{+}=0 \% \pm 0\right)($ Fig. $25 \mathrm{~A}, \mathrm{C}$ and $\mathrm{E} w t)$. In reeler, ectopically located E15.5-FT labeled neurons, still do not show a clear cell identity. Nevertheless, the percentage of cells positive for the upper-layer marker has increased in rostral and caudal cortices ( $r l-/$-rostral: Satb2 ${ }^{+}$ $=9.41 \% \pm 6.88, r l-/-$ caudal: $\left.S^{2} a t b 2{ }^{+}=15.14 \% \pm 9.62\right)$ and $\mathrm{FT}^{+}$cells remained largely Ctip2 negative $(r l-/-$ rostral: Ctip2 $^{+}=2.17 \% \pm 1.08$, rl-/-caudal: Ctip2 $^{+}=2.94 \% \pm 1.13$ ) (Fig.25 B, D and E rl-/-).

Thus, while in wild type animals E15.5-born cells gain the fate they are destined to, meaning the molecular identity of layer II/III neuron, in the absence of Reelin, ectopically located E15.5-born cells remain moleculary "fateless". 
wt
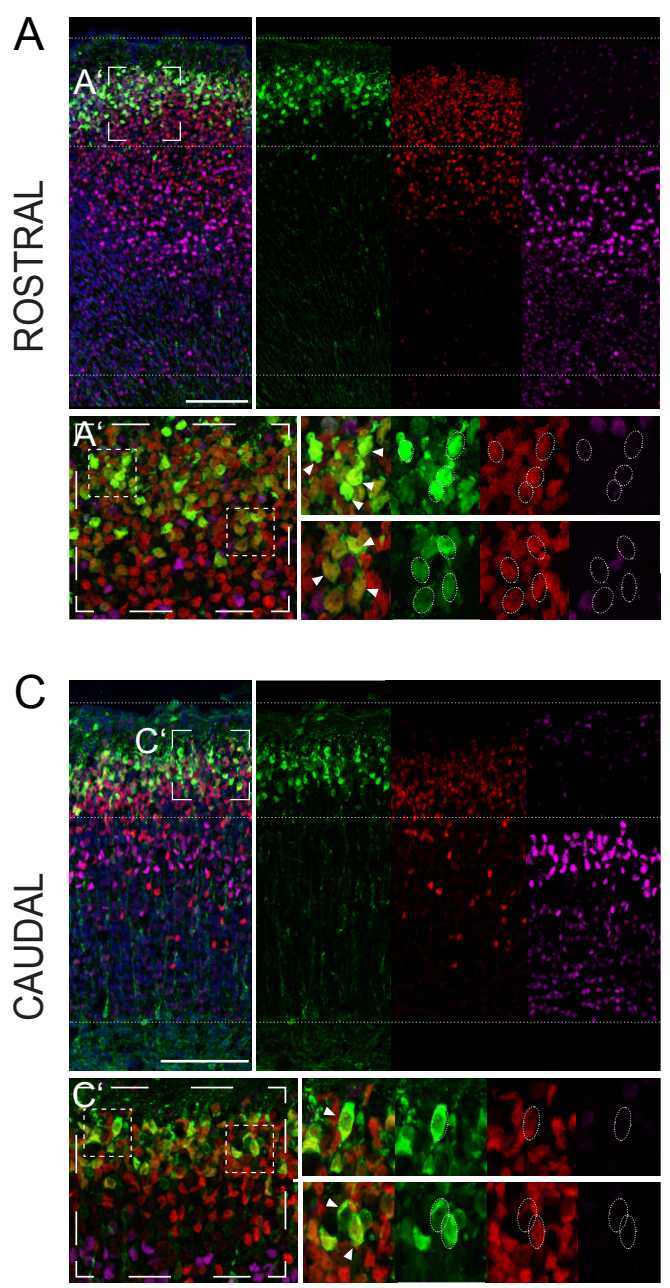
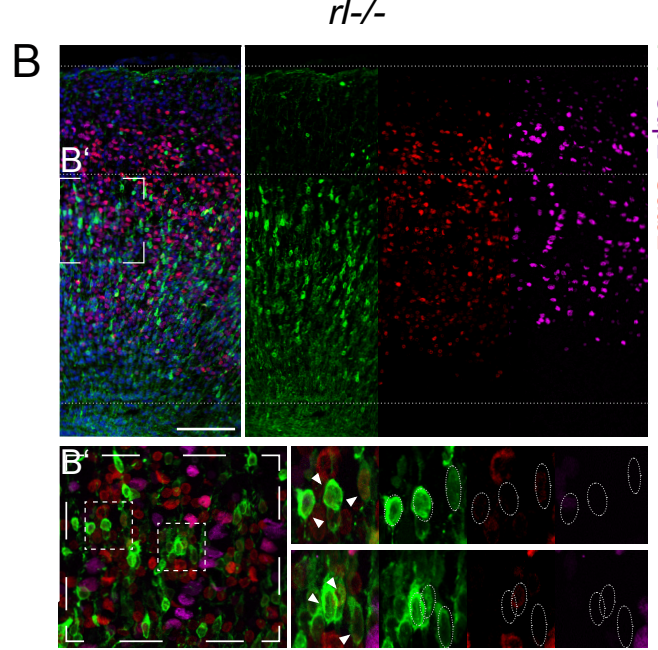

\section{$\mathrm{D}$}
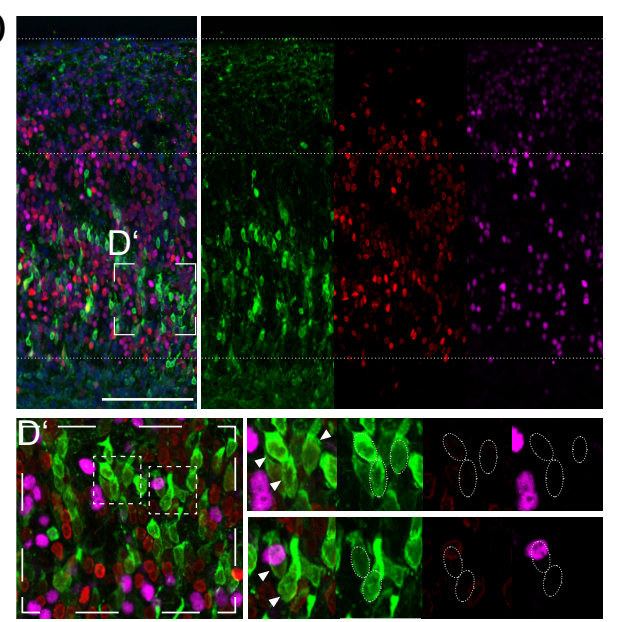

\section{$E$}
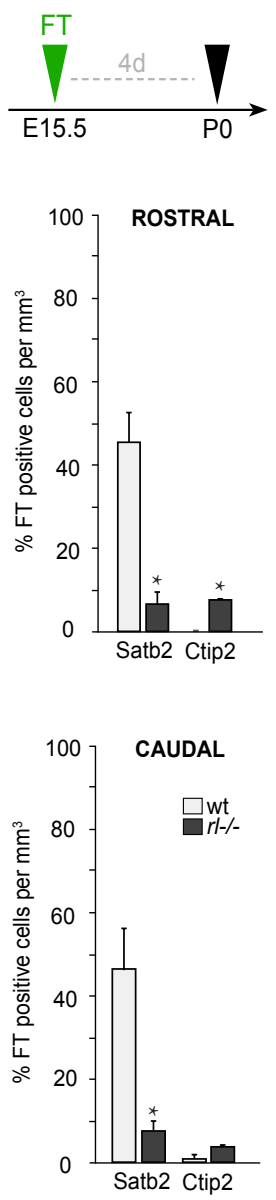

Figure 24. Cell fate of layer II/III cells in newborn wild type and reeler mice. E15.5 pups were subjected to a FTinjection, with the purpose of labeling supragranular cells. Triple immunostaining for FT (in green), Satb2 (in red, marker for upper layer-neurons) and Ctip2 (in magenta, marker for lower layer-neurons) on rostral (A, B) and caudal (C, D) wt and rl-/- coronal sections, four days post-injection. Dotted lines delineate LII/III, LIV-LVI and white matter. Experimental principle and quantification of FT, Satb2 and Ctip2 co-labeling in wt and $r$ l- $/-(n=2)$ (E). $\mathrm{FT}^{+}$cells in wild type sections $(\mathrm{A}, \mathrm{C})$ are progressively gaining the upper-layer fate $\left(\mathrm{Satb} 2^{+}\right)$and did not show immunoreactivity for the lower-layer neuronal marker (Ctip2) (arrowheads in $A^{\prime}$ and $C^{\prime}$ ) (E wt). Conversely, in the Reelin-deficient neocortex (B, D), cells do not show any preference for a certain molecularly-defined cell fate, neither in rostral nor in caudal cortices (arrowheads in $B^{\prime}$ and $D^{\prime}$ ) (E rl-/-). Scale bars: $100 \mu \mathrm{m}$. 

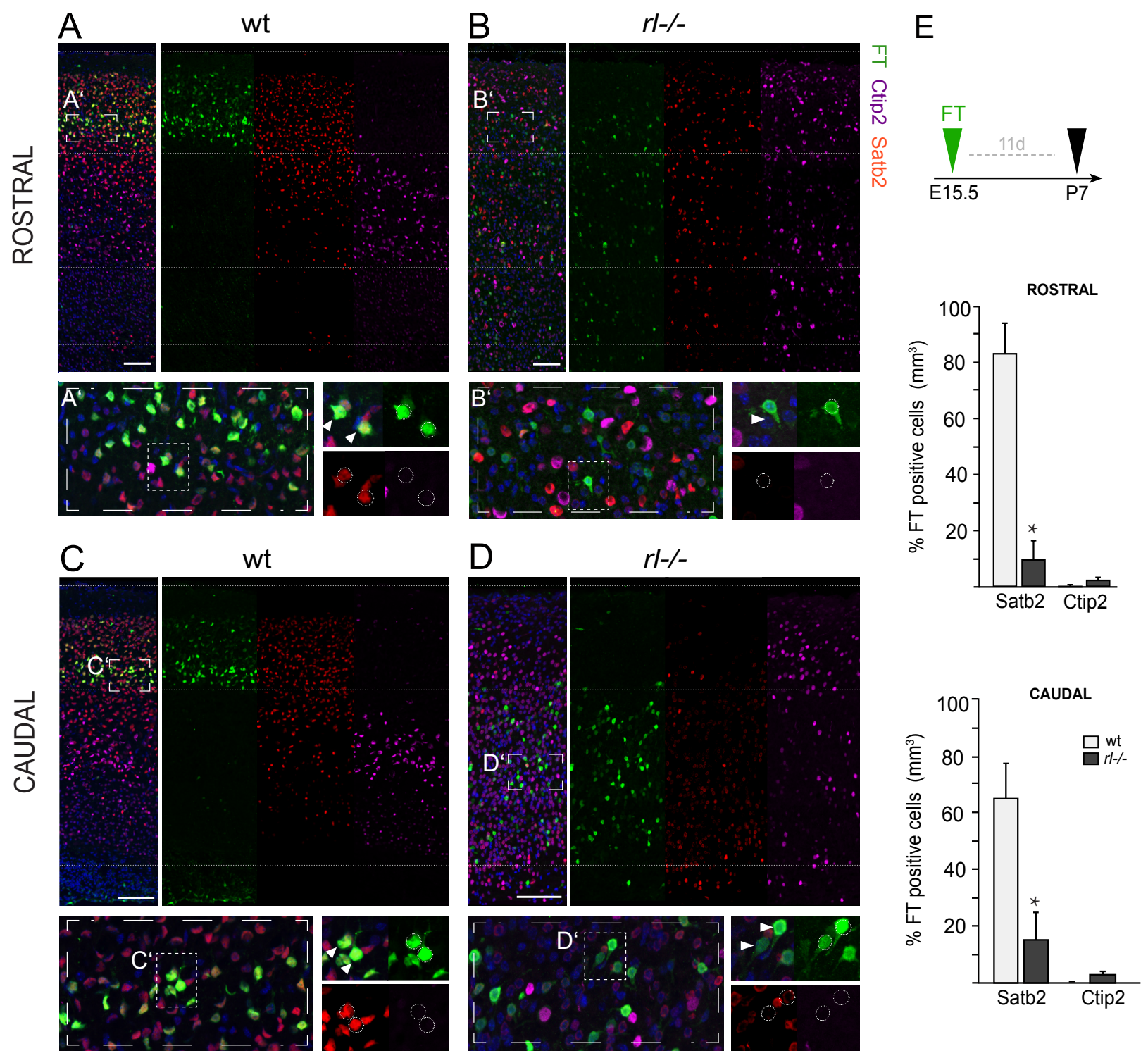

Figure 25. Cell fate of layer II/III cells in P7 wild type and reeler mice. VZ-derived supragranular cells were tagged by an E15.5-FT injection. Triple immunostaining for FT (in green), Satb2 (in red, marker for upper layerneurons) and Ctip2 (in magenta, marker for lower layer-neurons) on rostral (A, B) and caudal (C, D) P7 wt and rl-/- coronal sections, eleven days post-injection. Dotted lines delimit LII/III, LIV-LVI and white matter. Experimental principle and quantification of FT, Satb2 and Ctip2 co-labeling in wt and $r /-/-(n=2)(E)$. E15.5-FT cells in wild type sections $(A, C)$ are largely Satb2-positive and Ctip2-negative (arrowheads in $A^{\prime}$ and $C^{\prime}, E w t$ ). However, in the Reelin-deficient neocortex $(B, D)$, although there is certainly a preference for Satb2 expression, cells continue to be largely "fateless" (arrowheads in B' and D', E rl-/-). Scale bars: $100 \mu \mathrm{m}$. 


\section{HOW DO WE GET HERE? MORPHOLOGY OF LATE-BORN MIGRATING NEURONS}

Based on the lamina- fate marker approach on adult tissue, we could show that layer II/III cells are distributed in a gradient (high density close to pia and low density close to white matter) in the primary motor cortex (rostral), while they occupy deeper parts of the primary visual cortex (caudal). Pulse chasing analysis by halogenated thymidine analogues combined with Flash Tag labeling revealed that, two days after the pulse late-born, neurons are restricted to the IZ and migrate in as coherent population in both genotypes. However, migration of late-born neurons in reeler seems to be compromised, in an area-dependent manner, once the cells reach the cortex. The study of the relative location of a particular cluster of labeled cells gives us a valuable information about the migration kinetics and narrows the window at which the abnormalities start to become obvious in a Reelin-deficient environment. However, the morphology of those migrating neurons has also something to tell. Neuronal migration routes are dynamic and complex, as they involve changes in cell orientation and morphology. Those routes are also known to be influenced by Reelin at various levels (D'Arcangelo, 2014; Frotscher and Zhao, 2010). Neurons that migrate radially in the neocortex (e.g. LII/III cells) exhibit four main phases of migration (Nadarajah and Parnavelas, 2002): 1.) fast radial migration to the SVZ, 2.) migratory arrest in the SVZ, 3.) retrograde migration towards the apical VZ, and 4.) migration to the cortical plate, that ends in the detachment from the glial scaffold and neuronal differentiation. Cells are bipolar in phases 1, 3 and 4, whereas they are largely multipolar in phase 2. Phases 2 to 4 were evaluated in anterior and posterior regions in the Reelin-deficient neocortex by making use of the in utero electroporation (IUE) and Flash Tag (FT) techniques.

\section{Early stages of migration}

Migration deficiencies and morphological abnormalities (if any) at early stages of migration were assessed on E15.5 (pCAG-EGFP) in utero electroporated neurons. Embryos were harvested two days later (E17.5) (Fig.26). The orientation of the electrodes ensures the targeting of a desired brain region, in this case, M1 and V1 of the neocortex (Fig. 26 B). Reelin has been shown to regulate the switch from the multipolar to bipolar stage of immature projection neurons by cytoskeleton rearrangements. It seems also to encourage neurons to migrate towards the pial surface (Frotscher and Zhao, 2010). However, the morphology of the GFP-transfected cells in the 
VZ/SVZ and the IZ (Fig.26 C) in the Reelin-deficient neocortex mirrors wild type neurons (Fig. G, H). Surprisingly, in rostral and caudal cortical sections of reeler embryos, cells in the SVZ remain multipolar as they do in wild type animals. Moreover, cells in the IZ display the typical bipolar morphology of a migrating neuron (Fig. $26 \mathrm{H}$ ), with a long leading process at the front and a thin trailing process at the rear, similar to wild type brains (Fig.26 G). However, some migrating neurons exhibit an atypical morphology in the mutant mouse, as they have several processes (especially in caudal cortices) or an inverted orientation (Fig.26 H'). When a neuron has several branches, the centrosome and the Golgi apparatus are translocated to the basal portion of the dominant process, which becomes the dominant branch, named as the apical or leading process. The leading process determines the direction of migration of the neuron. Therefore, to assess cortical plate-orientation, we examined the location of the cis-Golgi, identified by immunostaining for GM130 (Horton et al., 2005; Nakamura et al., 1995) in combination with the orientation of the leading process in GFP-transfected neurons of both genotypes. The normal cortical orientation is maintained in the Reelin-deficient condition, as the majority of the neurons (> 80\%) migrate towards the cortical plate, both in rostral and caudal regions, as they do in the wild type littermates (Fig.26 E).

In conclusion, in the absence of Reelin late-born neurons adopt a multipolar morphology, transition back to a bipolar shape and display a pia-orientation, similar to the wild type littermates.

\section{Late stages of migration}

The orientation and morphology of late-born neurons at late stages of migration was further evaluated by Flash Tag. In the in utero electroporation technique, the application of electrical pulses makes the cell membrane temporarily porous, allowing its transfection by a particular plasmid. Those electrical pulses also cause disturbances in the embryos, which in a weak organism like reeler will certainly lead, in most of the cases, to embryonic death. The FT technology causes less stress to the embryos (as does not require the application of electrical current), labels the progeny of a given progenitor pool and postmitotic cells retain the dye for several days, making them suitable to be tracked during development.

The future layer II/III cells were labeled by FT at E15.5 and pups were harvested 2 days (E17.5), 4 days (P0) and 11 days (P7) after the injection (Fig.27). The pia-orientation was evaluated as described above. In the Reelin-deficient neocortex, when late-born migrating neurons are about to enter the 
cortical plate (E17.5), E15.5- FT labeled neurons show a clear preference to be oriented towards the pia surface, resembling the wild type situation (Fig.27 B). These neurons have a neat leading process, pointing to the marginal zone (Fig. 27 C), with the cis-Golgi located basally (Fig.27 B' and B"). Four days later (PO), neurons in the wild type cortex have settled down in a layer beneath the pial surface, with their leading processes contacting the marginal zone. In reeler, the prospective layer II/III cells, are still bypassing preborn cohorts of neocortical cells and remain still largely bipolar (Fig.27 D). Most of the $\mathrm{E} 15.5-\mathrm{FT}^{+}$cells have a pia-directed radial process, with the cis-Golgi situated basally (Fig.27 $\mathrm{D}^{\prime}$ and $\mathrm{D}^{\prime \prime}$ ), indicating they are heading toward the outer brain surface, in rostral and caudal sections. However, the absence of Reelin leads also to disorientation, as the apical dendrite of some $\mathrm{FT}^{+}$cells loses the straight orientation towards the pia mater, even exhibiting an inverted orientation (Fig.27 E). At P7, when radial migration is completed, projection neurons start to differentiate at their final destination (Fig.27 F). In reeler, the ectopically located neurons have visibly lost their polarity orientation, as indicated in previous studies (Chai et al., 2015; Goffinet and Lyon, 1979; Tabata and Nakajima, 2002) (Fig.27 F rl-/- and G). In rostral cortices, late-born neurons stabilize their apical dendrites downwards (empty arrowheads) but also in a normal upwards direction (Fig. G, filled arrowheads). Defects are more severe in caudal sections. Here, neurons generally fail to direct the apical dendrite towards the brain surface, and instead it is extended to variable and unpredictable directions (Fig. G, empty arrowheads).

Thus, over the antero-posterior in the Reelin-deficient neocortex, late-born neurons are able to maintain their pia-oriented migration at least upon entering the cortical plate. However, the absence of Reelin also entails, to a different extend in rostral and caudal sections, neuronal disorientation and deficits in the dendritic outgrowth. 
A
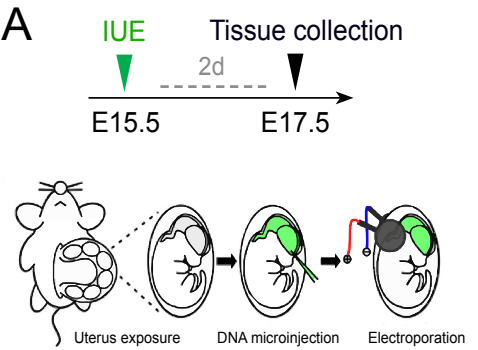

B

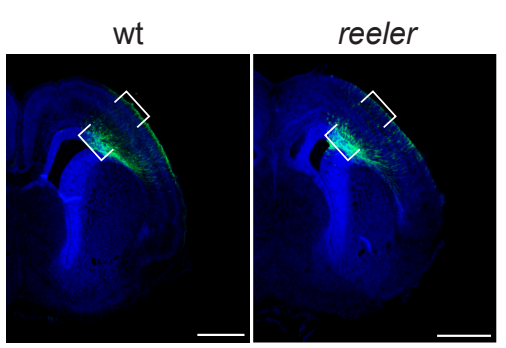

rl--
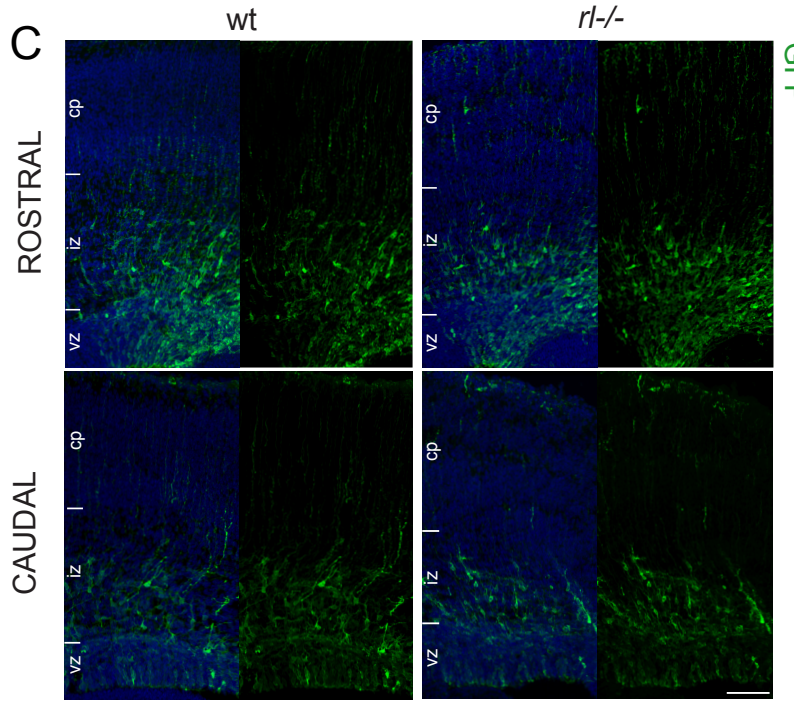

wt

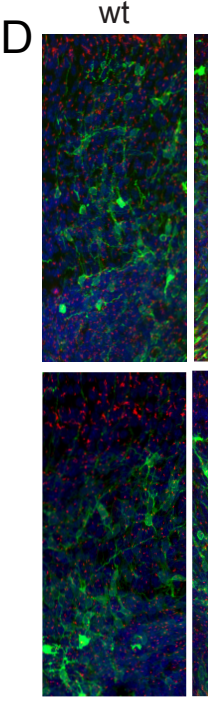

CAUDAL

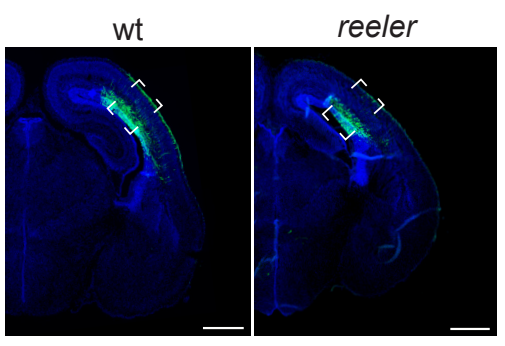

rl-/-

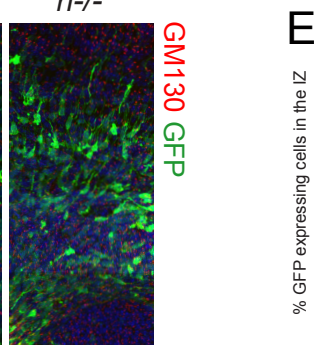

E

ROSTRAL

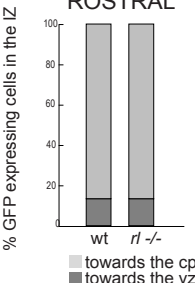

CAUDAL

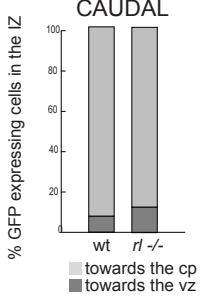

F

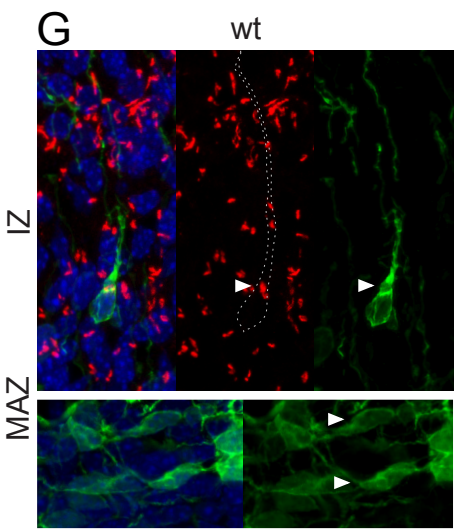

$\mathrm{H}$

rl--
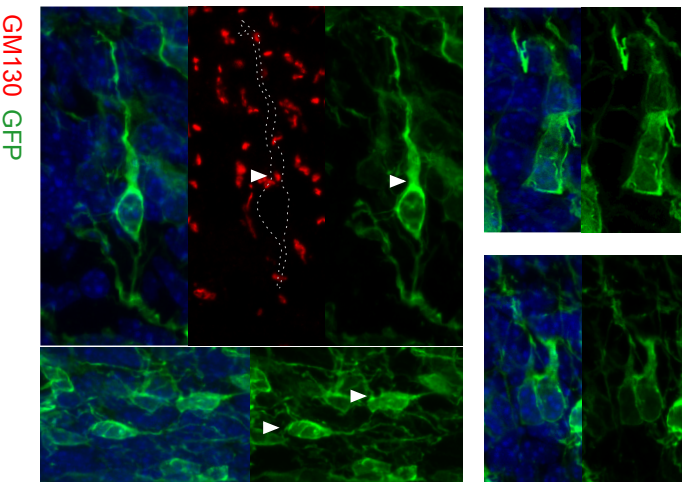

$\mathrm{H}^{6}$
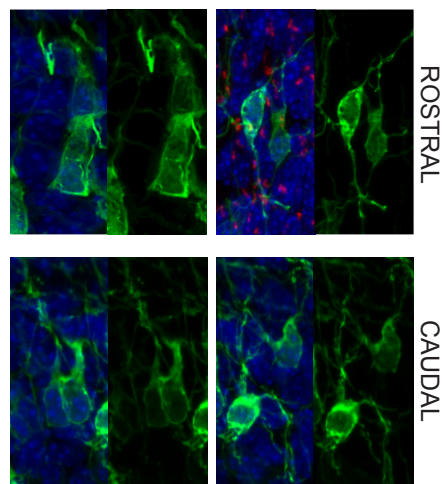

Figure 26. Morphology of late-born neurons appears normal at early stages of migration. Experimental design and in utero electroporation principle (modified from (Okada et al., 2007)) (A). GFP transfected (E15.5) cortical neurons in rostral and caudal (B-D) E17.5 wt and rl-/- sections. Golgi-apparatus was detected by GM130 immunostaining (shown in red $-D, G, H$ ). Direction (VZ-oriented) of migrating neurons in the $I Z$ is represented in $E(n=2,200$ neurons were counted). Main stages of cortical migration adopted by late-born neurons and phase of interested framed in (F). Two days after electroporation, morphology and orientation of GFP-transfected cells, in rostral and caudal cortices in reeler, mirrors the wild type situation. Abbreviations: $\mathrm{cp}$, cortical plate; iz, intermediate zone; vz, ventricular zone; maz: multipolar accumulation zone. Scale bars: $500 \mu \mathrm{m}$ (B) $100 \mu \mathrm{m}$ (C). 


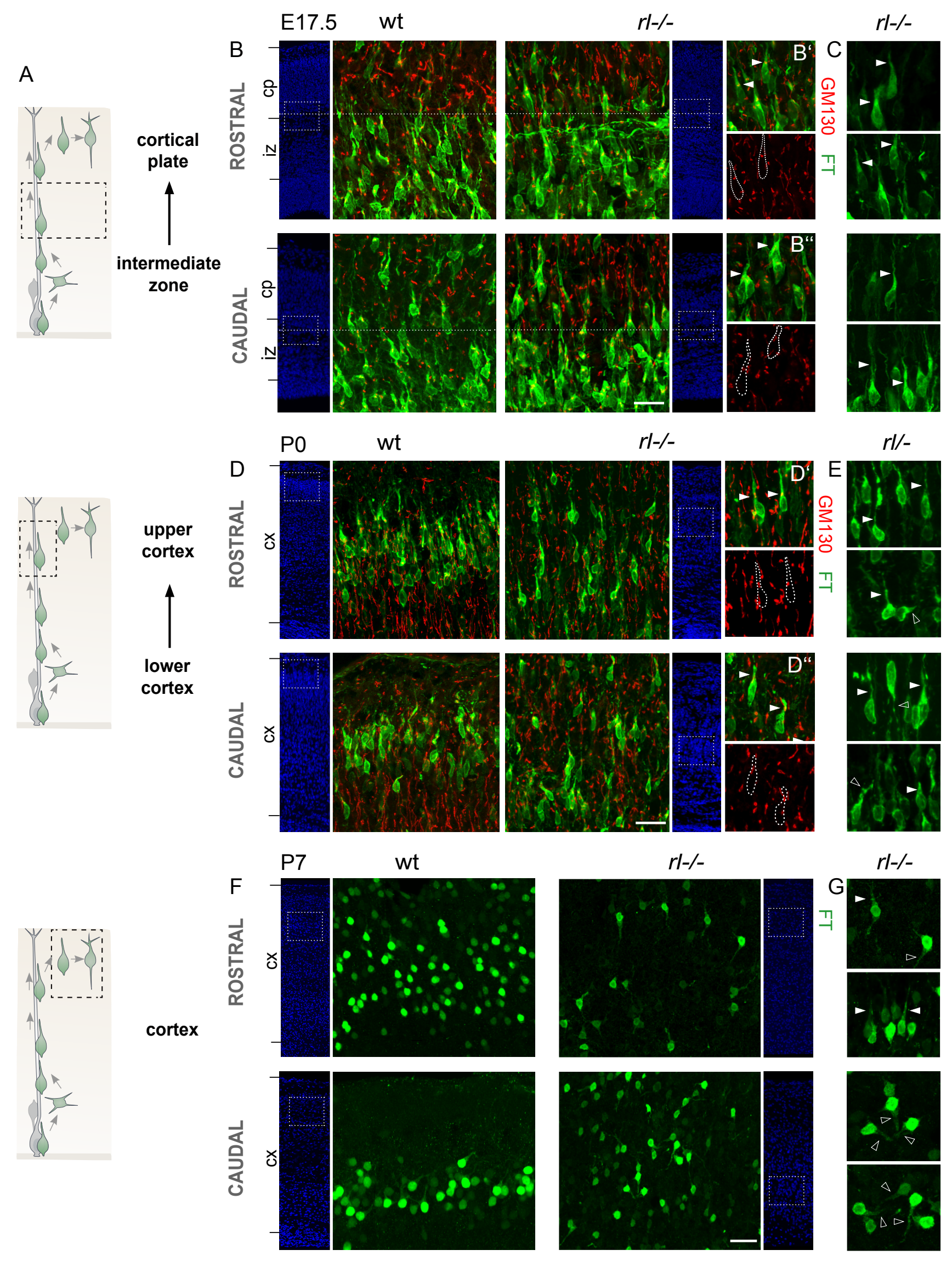


Figure 27. Morphology of late-born neurons is affected while sailing through the cortex in the Reelin-deficient neocortex. VZ-derived supragranular cells were tagged by an E15.5-FT injection and analyzed $2(B, C), 4(D, E)$ and $11(F, G)$ days post-injection. Main stages of cortical migration adopted by late-born neurons and phases of interest framed in (A). DAPI staining on a representative section for each developmental time point and genotype, and FT and GM130 (B-E) or FT only $(F, G)$ immunostaining for the corresponding regions of interest. Dotted lines delimit the $\mathrm{IZ}$ and $\mathrm{CP}$ in (B). Leading process and/or Golgi apparatus are marked by arrowheads. In reeler, most of the $\mathrm{FT}^{+}$cells have a pia-directed radial process upon entering the developing cortical plate. However, the absence of Reelin leads also to mild (rostral) and severe (caudal) disorientation as neurons migrate through the cortex. Immature neurons project apical dendrites towards unpredictable directions. Abbreviations: $c p$, cortical plate; iz, intermediate zone; cx, cortex. Scale bars: $50 \mu \mathrm{m}$.

\section{GLIAL SCAFFOLD AND REELIN}

\section{Glial fibers}

Three main transient structures support radial migration at developmental stages: CR cells, SP cells and radial glial (RG) fibers. RG fibers play an essential role in guiding migration, as they serve as the scaffold that layer $\mathrm{V}$ to layer $\mathrm{II} / \mathrm{III}$ neurons use to reach their final location. Having a disturbed glial scaffold can lead to complications in the glia-guided migration mode, that may therefore derive in an abnormal neuronal location in the mature cortex. It is known that Reelin influences the development of the RG fibers and that its appearance in reeler is altered (Hartfuss et al., 2003; Hunter-Schaedle, 1997). However, due to a rather general description, it is still imprecise whether the disturbances are different along the rostro-caudal axis. To address this question, distribution of GLAST-immunostained and GFP-labeled RG fibers in E15.5 and E17.5 reeler pups were compared with the wild type littermates (Fig.28). In wild type, RGCs extend a long process to the marginal zone, especially straight in the CP (Fig.28 A', B' C and $D^{\prime}$ ), in rostral and caudal cortices, which serve as guides for early- (at E15.5) and late- (at E17.5) migrating neurons (Fig.28 A, B, C and D wt). In reeler, GLAST ${ }^{+}$fibers are directed to the pial surface but their distribution is affected at E15.5 (Fig.28 A", B" rl-/-) and also at E17.5 (Fig.28 C", D" rl-/-). In the cortical plate, fibers meander and have slightly oblique angles. Fibers seems to particularly wander in caudal sections (Fig.28 B" and D"), which may contribute to the area-specific migration and location deficiencies. Upon contacting to the Reelin-enriched area, glial fibers develop multiple branches in wild type, seen as a dense GFP+ fiber region in the $\mathrm{MZ}$ (Fig.28 E, F wt; $\mathrm{E}^{\prime}$ and $\left.F^{\prime}\right)$. However, the branching of the basal processes of RGCs is drastically decreased in embryonic reeler mice, both in rostral and caudal sections (Fig.28 E, F rl-/-, E" and F"). 
E15.5

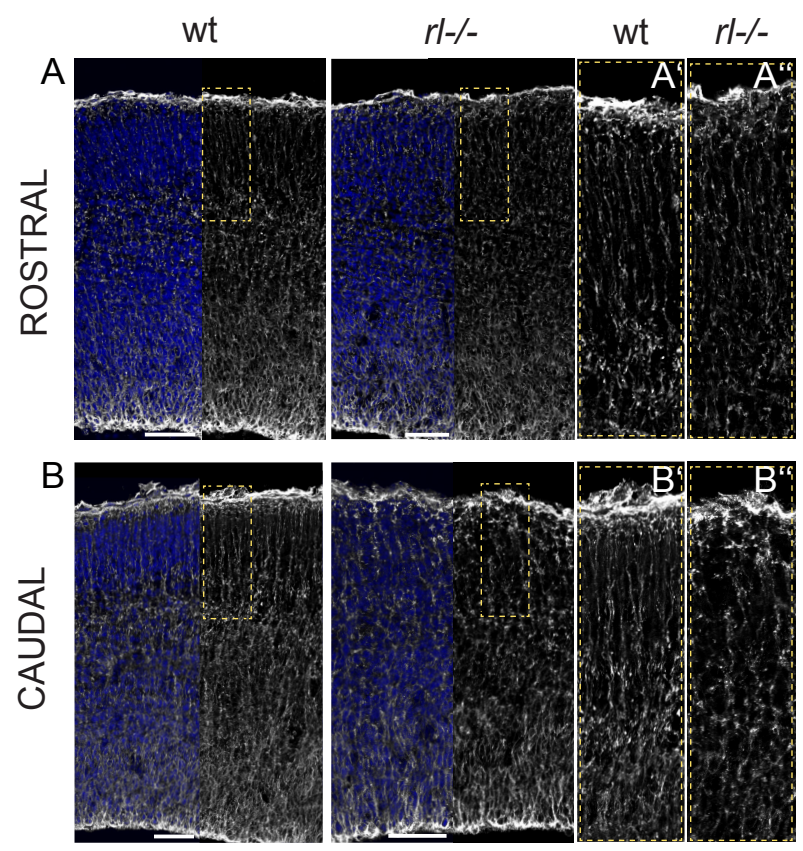

E17.5

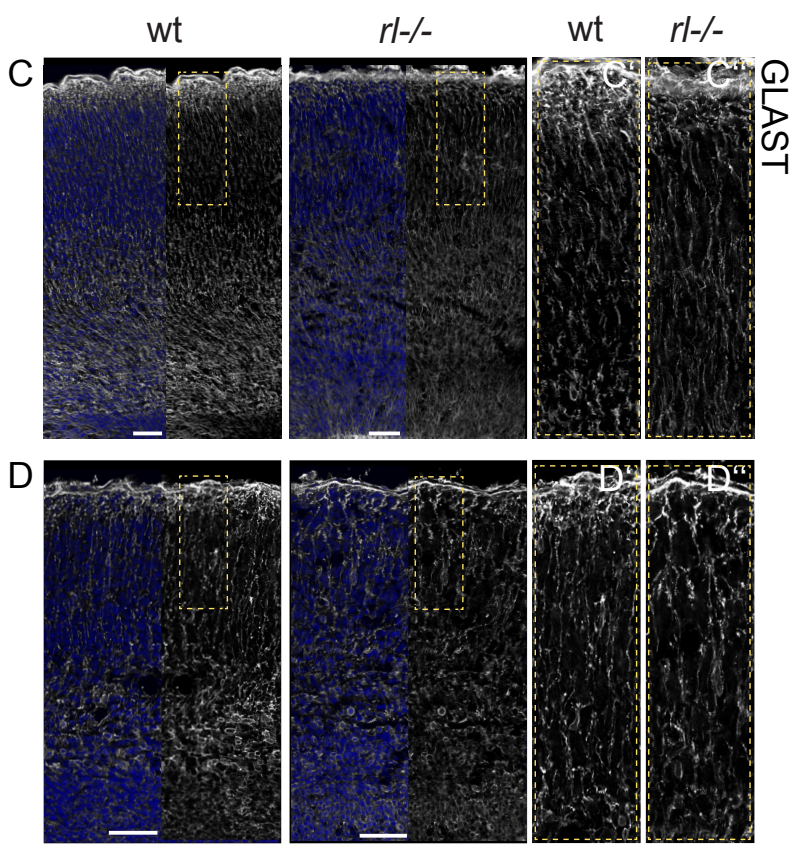

rl-/-
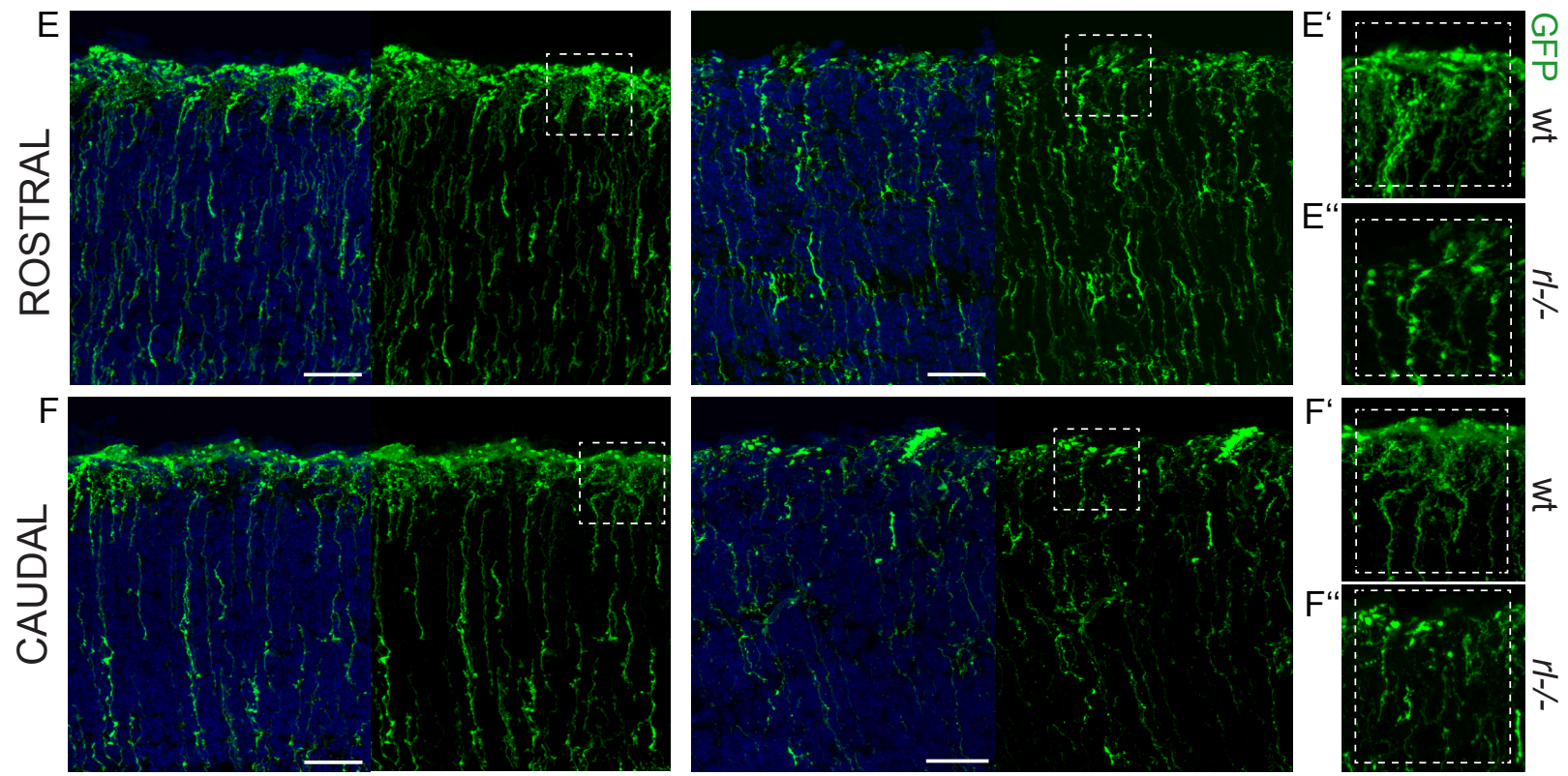

Figure 28. Radial glial fibers in E15.5 and E17.5 wild type and reeler mice. Immunostaining for GLAST on coronal sections from E15.5 and E17.5 normal and Reelin-deficient pups (A-D). GFP immunostaining on sectioned pCAX electroporated (at E15.5) E17.5 brains (upper cortical plate, E-F). Rostral and caudal cortices from both genotypes were examined. In wild type, RGCs extend a long process to the marginal zone, used as a guide for migrating neurons, which is abundantly branched at its end-feet. In reeler, radial fibers show a pronounced meandering, particularly in caudal regions. The terminal branching is critically reduced in the mutant mice. Scale bars: $50 \mu \mathrm{m}$. 


\section{Reelin expression over development}

Reelin is a fundamental player in guiding radial neuronal migration and its expression is considered to be necessary to promote cortical lamination in an inside-out manner. The proteolytic cleavage of the full-length protein produces different fragments with different diffusion properties, constituting different gradients from the MZ to the VZ (Jossin et al., 2007). However, apart from a brief description by (Ikeda and Terashima, 1997), where they suggested a slightly increased in the expression of reelin in rostral regions, there are no studies that approach the expression of Reelin (neither as the protein nor at the mRNA level) over the rostro-caudal axis. Thus, in order to investigate whether Reelin expression varies between areas through cortical development, its expression pattern was briefly evaluated in rostral and caudal cortices of wild type mice by Reelin immunostaining and reelin and Trp73 in situ hybridization (Fig.29).

At E15.5, a thin layer of cells, located beneath the pia and that covers the entire hemisphere, show immunoreactivity for the CR-50 antibody (a classical marker for the detection of the Reelin protein), in equal proportion in rostral and caudal sections (Fig.29 A). An equivalent scenario is seen when comparing the signal from the FISH for Trp73, as Trp73-expressing CR cells are similarly distributed covering in the outermost region of the developing brain, in anterior and posterior regions (Fig.29 B). Thus, this results seem to indicate that there are no obvious differences in the concentration of Reelin or CR cells when comparing rostral and caudal sections of a wild type E15.5 embryo.

reelin mRNA expression was further evaluated over development by $\mathrm{CISH}$. Rostral and caudal cortical sections from E13.5, E15.5, E17.5 and P0 wild type mice were included in the analysis (Fig. 29 C-F). At E13.5, when the cortical plate is just about to emerge, there is a high reelin expression in the $\mathrm{MZ}$ (black arrowheads), which in rostral sections is accompanied by a weak-expression band underneath it (white arrowhead) (Fig. 29 C). From E15.5 to E17.5 reelin mRNA is mainly restricted to the MZ, in rostral and caudal sections. The concentration seems to be slightly higher in caudal regions, although it is difficult to judge. The signal can be also detected throughout the cortical wall (Fig. 29 D, E). At P0, reelin expression is slowly shifted from CR cells ( $\mathrm{MZ}$, back arrowheads) to interneurons (cortex, white arrowheads), as described in the literature (D'Arcangelo, 2014; Pohlkamp et al., 2014). Notice that level of expression is presumably higher in rostral sections (Fig. 28 F). Thus, the data suggests there could be a rostro-caudal gradient in terms of reelin expression. However, more quantitative experiments (for instance, Western blot) are needed to validate whether Reelin acts in a gradient-fashion during development. 

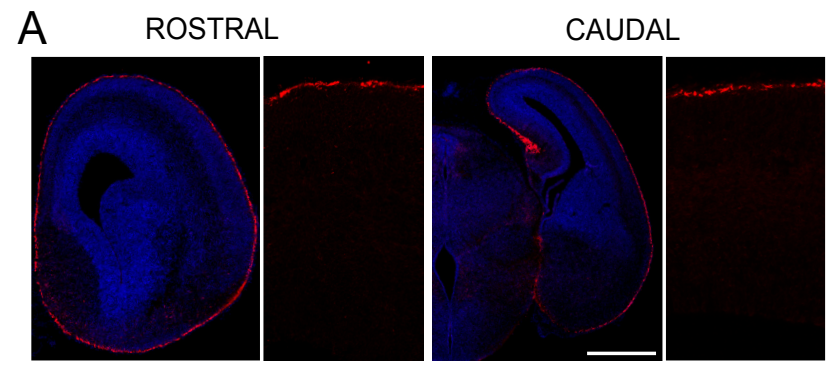

B
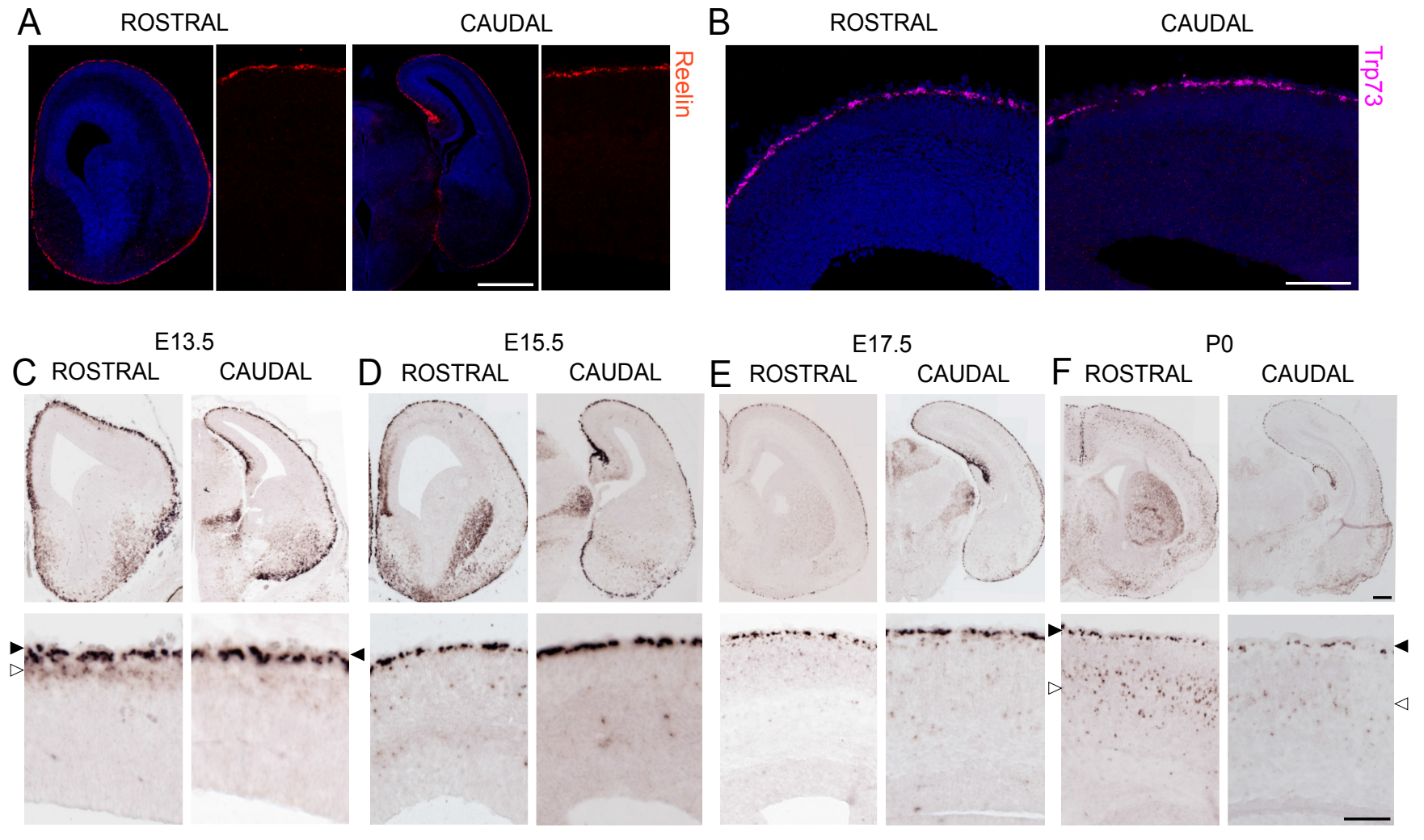

P0

CAUDAL

Figure 29. Reelin expression in anterior and posterior sections of wild type brains. Immunostaining for Reelin (E15.5, A), fluorescence ISH for $\operatorname{Trp73}(\mathrm{E} 15.5, \mathrm{~B})$ and mRNA reelin detection by chromogenic ISH through development (E13.5-P0) (C-F). Marginal zone and extra-marginal zone expression of reelin are marked by filled and empty arrowheads respectively ( $C$ and $F$ ). No apparent rostro-caudal difference in Reelin expression or Cajal Retzius cells are observed when comparing rostral and caudal cortices. ISH suggests a rostro-caudal reelin mRNA gradient, whose validation demands additional experimental data. Scale bars: 250 (A, F) and 100 (B, F) $\mu \mathrm{m}$. 


\section{A BRIEF LOOK INTO REELIN-RECEPTORS}

Reelin is known to exert its action on neurons in the developing cortex through two main receptors: ApoER2 (apolipoprotein E receptor type 2, also known as Lrp8) and VLDLR (very low density lipoprotein receptor). Regardless of the suggested functional overlay, differences in the expression patterns (Benhayon et al., 2003; Hack et al., 2007b; Trommsdorff et al., 1999), distinctive affinities to Reelin (Andersen et al., 2003) and variations of the expression domains (Kim et al., 1996), suggest distinct physiological functions for VLDLR and ApoER2 in neuronal migration. In order to investigate the function of the receptors in specific clusters of migrating projection neurons, VLDLR and ApoER2 overexpression vectors were transfected into living cells by making use of the in utero electroporation technique.

pcDNA3.1-ApoER2 ${ }^{\text {Dyk }}$ (CMV-ApoER2 ${ }^{\text {Dyk }}$ ) and pcDNA3.1-VIdlr ${ }^{\text {Dyk }}$ (CMV-VIdlr ${ }^{\text {Dyk }}$ ) have a Flag tag (Dyk or DYKDDDDK tag) that can be tracked by using an antibody detection approach in cell culture or western blot, but lack the sequence for any reporter gene that facilitates visualization in vivo. Moreover, ApoER2 and VLDLR transgenes are expressed under the CMV promoter, and the expression of this particular promoter does not persist in postmitotic neurons. Thus, prior using for in vivo experiments, coding sequences were subcloned into a plasmid, named pCIG2 (pCAG-IRES-EGFP), more suitable for our goal. pCIG2 drives the expression of eGFP and the gene of interest downstream of the CAG promoter. The CAG promoter provides a strong expression that drops rapidly in the VZ after the electroporation (providing a birth date specific expression) and persists in postmitotic neurons. It can be used for both, in vitro and in vivo studies. The

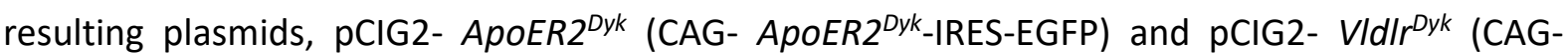

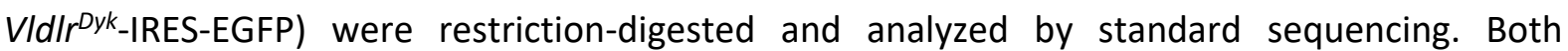
approaches showed the inserts to be successfully ligated in the correct orientation (data not shown).

The protein expression of the resulting plasmids was tested in cell culture and western blot (Fig.30). HEK293T (human embryonic kidney cells- 293) or Neuro2A (fast-growing mouse neuroblastoma cell line) cells were transfected with pcDNA3.1-ApoER2 ${ }^{\text {Dyk }}$ (shown as pcDNAApoER2 ${ }^{\text {Dyk }}$ ), pcDNA3.1-VIdlr ${ }^{\text {Dyk }}$ (shown as pcDNA-VIdlr ${ }^{\text {Dyk }}$ ), pCIG2, pCIG2-ApoER2 $2^{\text {Dyk }}$ and pCIG2VIdIr ${ }^{\text {Dyk }}$ constructs. Two days after transfection, cells were subjected to protein extraction (and subsequent western blot analysis) (Fig.30 A-B) or immunocytochemistry (Fig.30 C-D). Protein 
expression of the pcDNA plasmids in cell lysates was validated by immunoblotting with the antiDyk antibody (tag). However, protein expression from pCIG2 constructs could not be validated by detecting the tag, neither for ApoER2 nor for VLDLR (Fig.30 A). Extracts were then subjected to a western blot analysis with anti-ApoER2 and anti-VLDLR antibodies. The expression of the protein ApoER2 was corroborated by application of the ApoER2 antibody, as all tentative constructs loaded gave a strong band, similar to the band in the control lane. The validation of the VLDLR expression remains elusive. Further experiments, e.g. new transfections, different antibody concentrations or even new anti-VLDLR antibodies, will have to confirm its expression by western blot (Fig. 30 B). Nevertheless, the immunocytochemistry of HEK293T or Neuro2A transfected cells did ratify that both $\mathrm{pCIG} 2$ plasmids drive the expression of the proteins of interest. Cells transfected with pCIG2- ApoER2 ${ }^{D y k}$ and pCIG2- VIdIr ${ }^{D y k}$ constructs could be detected by the antitag (Dyk) antibody (shown in red). These cells also expressed GFP (shown in green), facilitating the visualization of the transfected cells and its morphological features (Fig.30 C, D). Best-working overexpression candidates were then purified for in vivo experiments.
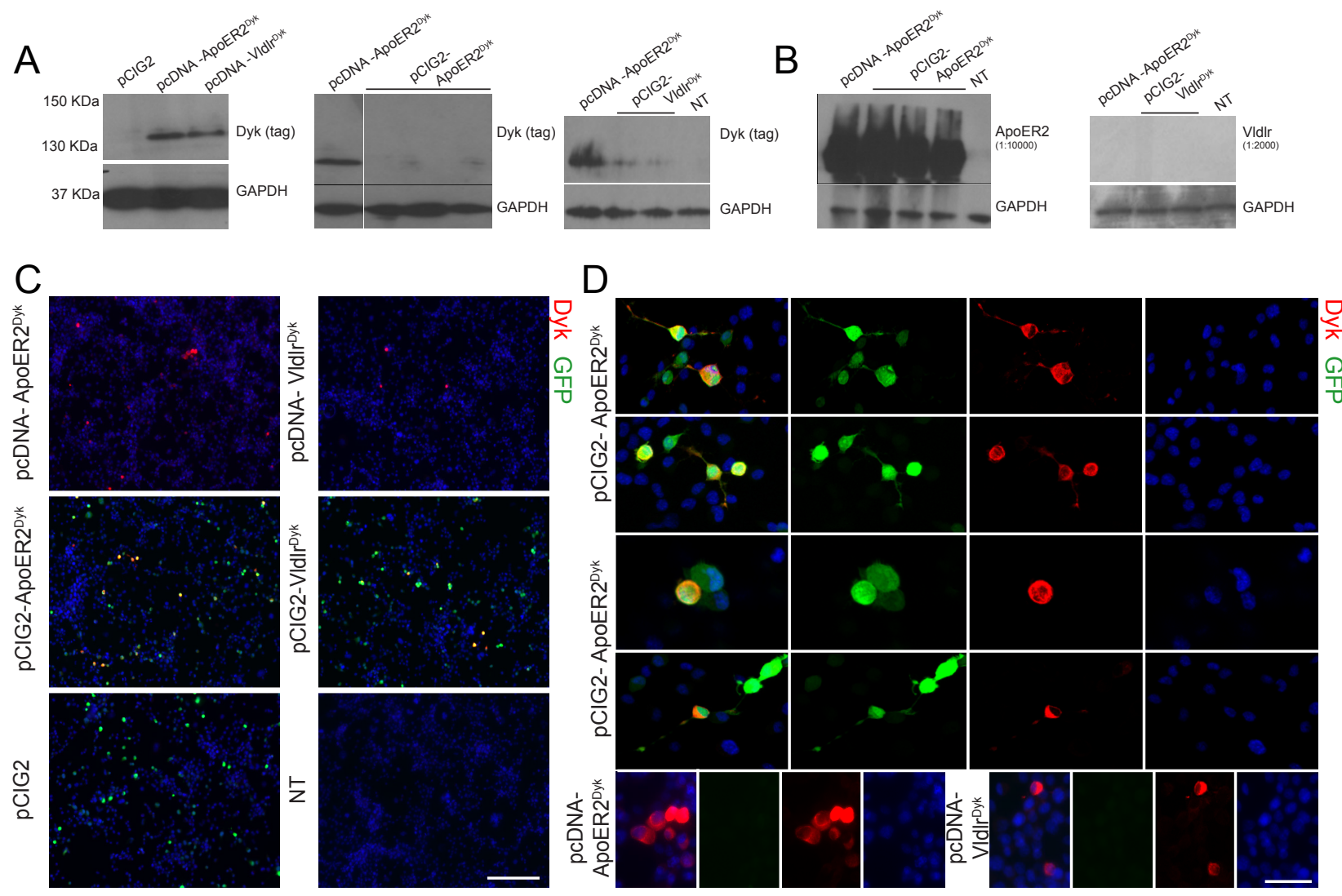

Figure 30. Validation of VLDLR and ApoER2 protein expression in pcDNA and pCIG2 constructs. Western blot analysis with anti- GAPDH, Dyk (tag), ApoER2 and VLDLR antibodies to validate VLDLR and ApoER2 expression in transfected-cell lysates. GAPDH used as loading control (A, B). Immunocytochemistry (ICC) in HEK293T or 
Neuro2A transfected cells (C, D). Plasmids used: pcDNA3.1-ApoER2 ${ }^{\text {Dyk }}$ (shown as pcDNA-ApoER2 ${ }^{\text {Dyk }}$; CMVApoER2 ${ }^{\text {Dyk}}$ ), pcDNA3.1-VIdll $r^{\text {Dyk }}$ (shown as pcDNA-VIdlr ${ }^{\text {Dyk; }}$ CMV-Vldll ${ }^{\text {Dyk }}$ ), pCIG2 (CAG-IRES-EGFP), pCIG2$A_{\text {ApoER2 }}{ }^{\text {Dyk }}$ (CAG- ApoER2 $2^{\text {Dyk}}$-IRES-EGFP) and pCIG2-VIdlr ${ }^{\text {Dyk }}$ (CAG-VIdlr ${ }^{D y k}$-IRES-EGFP). Protein expression of ApoER2 and VLDRL was validated by western blot and ICC, respectively. Abbreviations: NT, non-transfected cells. Scale bars: $200 \mu \mathrm{m}$ (C) and $50 \mu \mathrm{m}$ (D).

To investigate the potential role of the main Reelin receptors in the migration of cortical

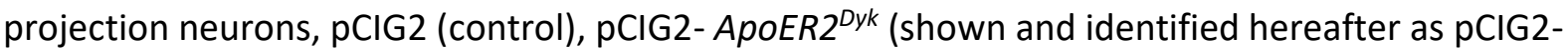
ApoER2) and pCIG2- VIdlr ${ }^{D y k}$ (shown and identified hereafter as pCIG2- VIdlr) constructs were introduced in E14.5 embryos by in utero electroporation. Pups were harvested one (E15.5) and two (E16.5) days after electroporation (Fig.31 and 32).

A day after electroporation (E15.5, Fig. 31), E14.5-transfected GFP-expressing cells are restricted to the VZ-MAZ zone in all conditions. However, the expression of ApoER2 (pCIG2ApoER2) seems to induce multipolar morphology while VLDLR (pCIG2-VIdIr) appears to encourage a bipolar shape (Fig. 31 B). Two days after electroporation (E16.5, Fig. 32), E14.5- transfected cells with the control plasmid $(\mathrm{pCIG2})$ are located in the vicinity of the cortical plate or already bypassing older cortical neurons. The overexpression of ApoER2 (pCIG2- ApoER2), however, clearly affects the migratory kinetics of electroporated cells, as GFP-expressing neurons have not arrived at the cortical plate and are found in the intermediate zone instead. VLDLR-expressing neurons (pCIG2- VIdrl) are preferentially located in upper levels in the cortical column, as they accumulate below the cortical plate or are migrating over previously born cortical cells by now. The number of $\mathrm{Tbr}^{+}$cells (marker for infragranular cells) in the cortical plate of ApoER2 transfected brains is clearly reduced, but its severity and cause has to be investigated further. The level of glia-neuron interaction could not be resolved by combining the GFP detection with a GLAST immunostaining (shown in magenta). Moreover, although ApoER2 transfected appear preferentially multipolar, higher resolution microscopy is needed to resolve the morphological features of these and VLDLR-expressing neurons (Fig.32 B). In summary, and until additional experiments are carried out, the data suggest that the overexpression of ApoER2 and VLDLR promotes a multipolar and bipolar morphology, respectively, which affect future steps of radial migration of projection neurons. 
A
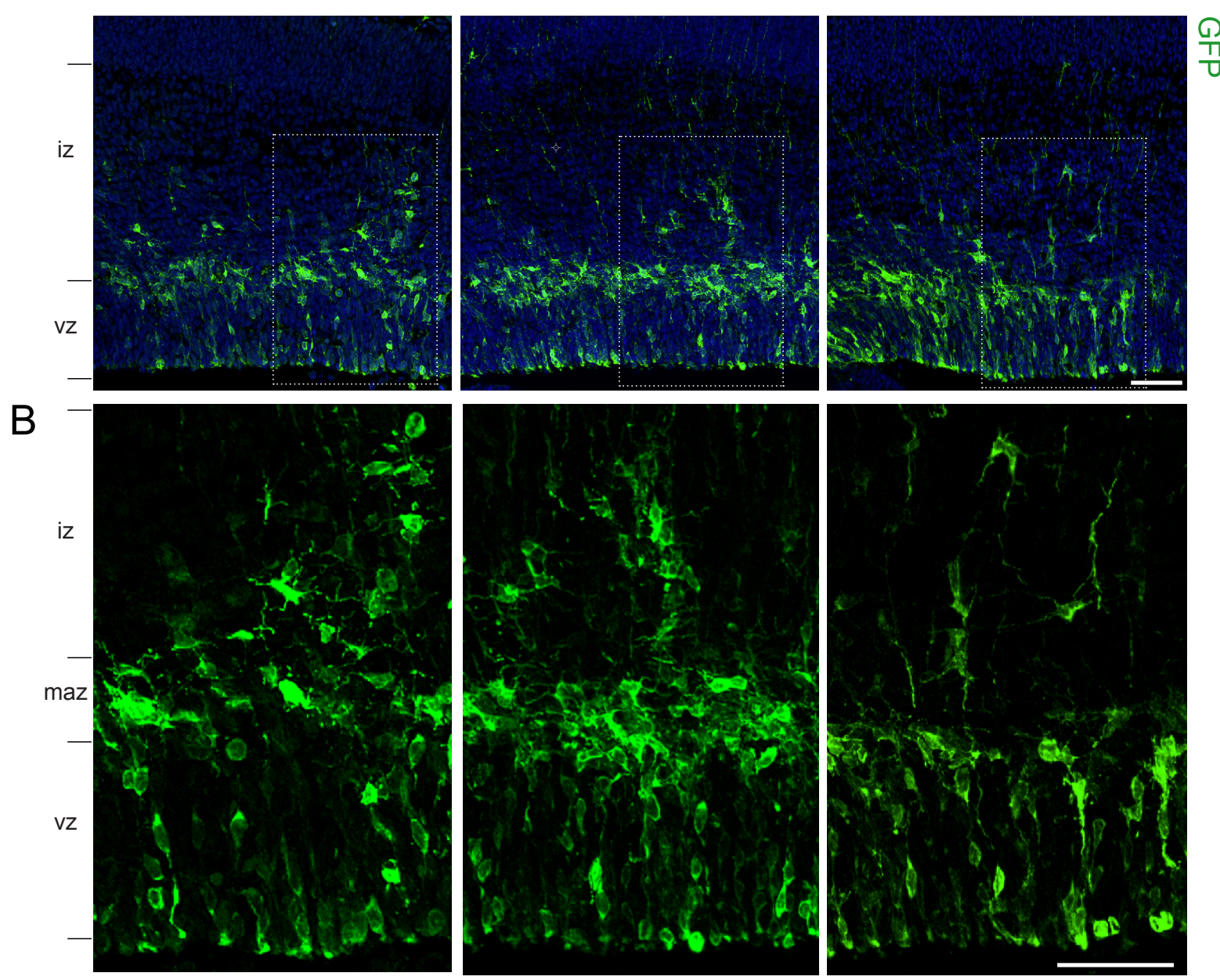

Figure 31. Overexpression of Reelin receptors in migrating neurons in the VZ-IZ. E14.5 CD1 embryos were electroporated with pCIG2 (control plasmid, CAG-IRES-EGFP construct), pCIG2- ApoER2 (CAG- ApoER2 ${ }^{\text {Dyk }}$-IRESEGFP construct, labeled as pCIG2-ApoER2) or pCIG2- VIdlr (CAG- VLDLR ${ }^{\text {Dyk }-I R E S-E G F P ~ c o n s t r u c t) ~ p l a s m i d s, ~ a n d ~}$ harvested a day later (E15.5). Immunostaining for GFP on medial E15.5 coronal sections (A,B). The expression of ApoER2 seems to drive a multipolar morphology while the expression VLDLR appears to induce a bipolar shape. Abbreviations: iz, intermediate zone; vz, ventricular zone; maz: multipolar accumulation zone. Scale bars: $50 \mu \mathrm{m}$. 

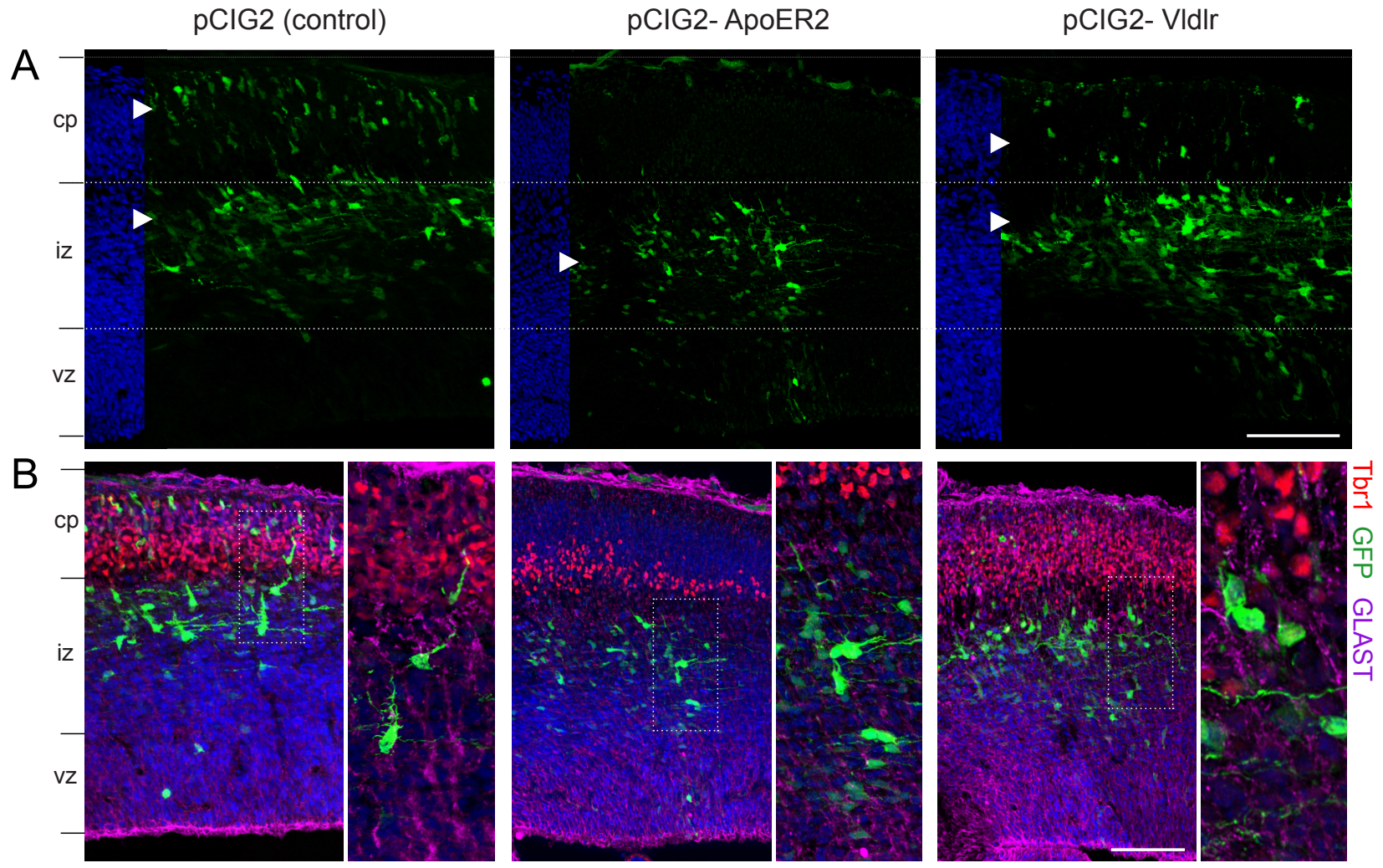

Figure 32. Overexpression of Reelin receptors in migrating neurons in the IZ-CP. E14.5 CD1 embryos were electroporated with pCIG2 (control plasmid, CAG-IRES-EGFP construct), pCIG2- ApoER2 (CAG- ApoER2 ${ }^{\text {Dyk }}$-IRESEGFP construct, labeled as pCIG2-ApoER2) or pCIG2- VIdlr (CAG- VLDLR ${ }^{\text {Dyk }}$-IRES-EGFP construct) plasmids, and harvested two days later (E16.5). Immunostaining for GFP, GLAST and Tbr1 on medial E16.5 coronal sections (A, $B)$. The overexpression of ApoER2 and VLDLR impairs radial migration as neither ApoER2- nor VLDLR-transfected cells resemble the distribution of GFP-expressing neurons in control brains (arrowheads). Dashed lines in (A) delimit the CP, IZ and VZ. Abbreviations: $c p$, cortical plate; iz, intermediate zone; vz, ventricular zone. Scale bars: $100 \mu \mathrm{m}$. 


\section{DISCUSSION}

\section{ADULT CORTICAL LAMINATION IN THE ABSENCE OF REELIN}

The fine-grained analysis of the neocortical cytoarchitecture shown here provides a detailed view of the complexity of the neocortex in the absence of Reelin. According to previous studies (Boyle et al., 2011; Caviness and Sidman, 1973), all major classes of cortical neurons (fated for a certain layer) are detected in the adult cortex in reeler, and their relative proportions are equivalent to those found in wild type animals. However, the lamina-fate marker approach carried out in this study demonstrates that the inverted pattern symbolizes an oversimplification, and it is far from representing the intricacy of the Reelin-deficient cortex. Instead, the reeler cortex is shaped in an area-dependent (mis)laminated fashion (Fig. 33). Cortical patterns in primary cortices range from a more quasi- wild type phenotype in the rostral motor cortex, via an almost layer disappearance in the intermediate somatosensory cortex, to a quasi- inversion phenotype in the caudal visual cortex.

Theories aiming to specify the role of Reelin have been strongly influenced by the interpretation of the reeler phenotype. The reeler neocortex is regularly described as a rough laminar inversion. The description of the inverted or outside-in laminar pattern, where early-and late- born neurons occupy upper and lower layers respectively, is based on low-resolution techniques that, although revolutionary at the time of their publication, did not allow the discrimination of specific cell types (Caviness, 1982a; Caviness and Sidman, 1973). Few studies have dared to question the "inverted cortex hypothesis" . For instance, it has been shown that the expression of certain molecular markers, such as Fez1 (specific for LV cells) and Cux2 (specific for LII/III-IV cells), is scattered throughout the reeler cortex (Ferrere et al., 2006; Inoue et al., 2004). Scattering of putative LV cells was also demonstrated by retrograde HRP labeling in the adult reeler neocortex (Dekimoto et al., 2010; Terashima et al., 1983). More detailed studies, based on the expression of layer-specific markers detected by in situ hybridization, proposed a spatial dispersion of ER81 (LV), Rorb (LIV) and Rgs8 (LII/III)- neurons in the motor cortex, somatosensory cortex and visual cortex of reeler mice (Dekimoto et al., 2010; Pielecka-Fortuna et al., 2014; Wagener et al., 2010; Wagener et al., 2016). Additionally, the Allen Institute investigated the consequences of the absence of Reelin on the lamination of the neocortex and other brain 
structures by making use of a large genome-scale in situ hybridization atlas. This time, authors suggested that the reeler cortex preferentially follows a mirror-image fashion (Boyle et al., 2011). These studies, however, either lack quantification or are bound to a certain layer or brain region. Moreover, they have not influenced enough the reception of the reeler cortex by the scientific community, as the concept of the inverted pattern still prevails nowadays (Chai and Frotscher, 2016; Cooper, 2008; Gupta et al., 2002; Jiang and Nardelli, 2016; Tissir et al., 2002). The study presented here shows, for the first time, a complete qualitative and quantitative analysis of the cellular distribution of the major neocortical layers, in areas that cover the antero-posterior axis of the reeler brain. The data demonstrates that the unique cytoarchitectural patterns of the sensorimotor areas outline an inside-out to an outside-in gradient, in a rostro-caudal manner. Thus, in the rostral motor cortex early- and late- born neurons are both found in upper and lower layers respectively, while in the caudal visual cortex these neurons are inversely located, more in line with the classical view. The frontal association cortex, despite a strong cellular intermingling, follows in general the rules from the gradient found in the sensorimotor areas, as the (broad) distribution of LII/III (Rgs)-neurons is followed by LIV (Rorb)- and layer V (Etv1)-fated cells. The frontal association cortex is engaged in stimulus integration (Nakayama et al., 2015), and receives sensorimotor input from other cortical areas. Thus, and given the particular functional logic of this cortical area, it would be of special interest to investigate in the future how the neocortex is shaped during development and how other delaminated sensory cortices influence its arrangement by activity-dependent mechanisms in reeler. Future studies have also to show how similar or dissimilar the frontal association cortex is to other associative cortical areas.

The regional cell positioning heterogeneity of the (mis)laminated patterns of the reeler cortex speaks against a general passive displacement of older neurons by successive waves of projection neurons. In such a scenario, LVI cells should have all remained above LII/III- to LV-fated cells and this is certainly not the case. Even though LVI ( $\left.T C_{1460681}\right)$ cells indeed stay superficially in all areas examined, these cells are also found close to the white matter in the primary motor and primary somatosensory cortex. On the other hand, the outside-in or inverted model implies that each wave of projection neurons fails to pass older cortical neurons, and thus differentiates below its predecessors. However, LII/III (Rgs8) cells are found in upper cortical regions in the frontal association and primary motor cortex, with cells fated to LIV (Rorb) and LV (Etv1) found beneath. LII/III cells are also above a quite large number of previously born neurons, meaning LIV, LV and/or $\mathrm{LVI}$, in the primary somatosensory and auditory cortex. Moreover, the delamination variability in 
the Reelin-deficient neocortex suggests that there is no uniform mechanism through which Reelin exerts its effect on corticogenesis along the rostro-caudal axis. Additionally, our data indicate that main neocortical regions or even cell types may respond differently to the absence of Reelin. The molecular mechanisms responsible for this differential cellular displacement need to be determined.

wild type

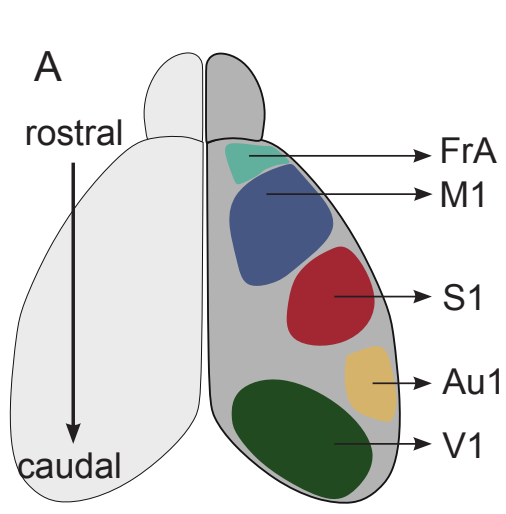

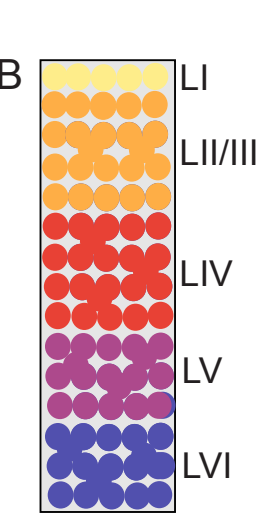

reeler

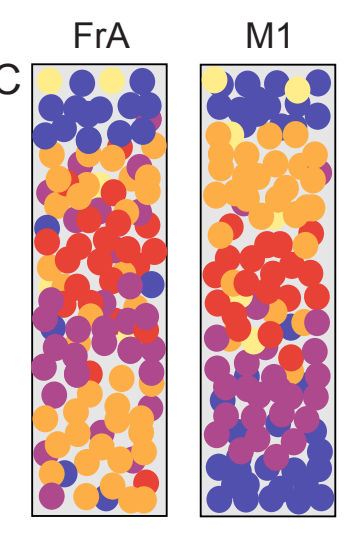

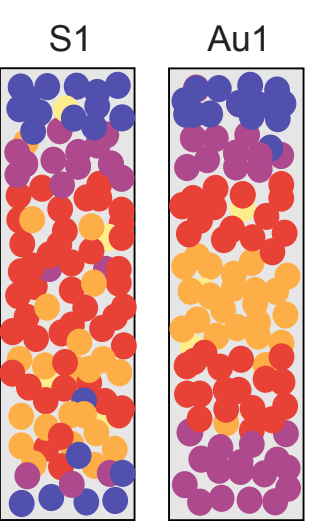

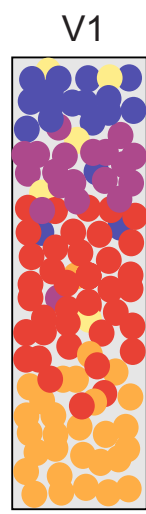

Figure 33. Area-dependent disruption of the neocortical lamination in Reelin-deficient mice. (A) Dorsal view of the adult brain in mice with a color-coded representation of the frontal association cortex (FrA), primary motor cortex (M1), primary somatosensory cortex (S1), primary auditory cortex (Au1) and primary visual cortex (V1). (B, C) Schematic representation of the cortical lamination in the wild type neocortex (B) and areas shown in $A$ in the Reelin-deficient mouse (C). The highly disturbed reeler cortex is not simply inverted but rather shaped in an area-dependent (mis)laminated fashion. Layers are represented as roman numerals: LI (yellow), LII/III (orange), LIV (red), LV (magenta), LVI (blue).

\section{PREPLATE SPLITTING}

The simultaneous detection of the main components of the preplate and the examination of the trajectories of the thalamocortical projections during embryonic development, indicate that preplate splitting is differentially disrupted over the rostro-caudal axis in the absence of Reelin. The inconsistency in preplate splitting inhibition may be considered the trigger to the quasi- wild type and quasi- inverted pattern in rostral and caudal regions, respectively, in the adult Reelindeficient neocortex. 
Preplate. The formation of the neocortical layering starts when the prospective layer VI cells divide the preplate into the superficial marginal zone and the deeper subplate (Marin-Padilla, 1978). The marginal zone is populated by Cajal Retzius cells, which constitute the major source of the extracellular matrix glycoprotein Reelin during corticogenesis (D'Arcangelo et al., 1995). The subplate zone is composed by subplate cells, which have crucial roles in development and brain maturation.

Cajal Retzius cells and subplate cells have multiple origin sites and undergo numerous developmental refinements (Garcia-Moreno et al., 2007; Montiel et al., 2011; Oeschger et al., 2012; Pedraza et al., 2014; Yamazaki et al., 2004), which makes their identification and tracking over development, particularly in the case of the subplate cells, fairly difficult. Subplate cells have been classically visualized by BrdU- birth dating, MAP2 or Tbr1 immunostaining in reeler and other mouse lines (Bayer and Altman, 1990; Nichols and Olson, 2010; Sheppard and Pearlman, 1997). However, these studies suffer from a lack of accuracy. For instance, BrdU injections at E10-E11 give a rather sparse cell distribution because, as BrdU is incorporated into the de-novo synthesized DNA in all dividing cells, it labels subplate cells but also projection neurons born after the pulse. Likewise, the expression of Tbr1 in subplate cells and early-born neurons prevents a discrimination of distinct cell populations (Hevner et al., 2001). It was just recently that a geneexpression profile from embryonic subplate cells became available (Oeschger et al., 2012). The authors also analyzed the expression of the newly described subplate-specific genes in reeler cortices and concluded that its expression was shifted. However, no further specifications are given about the examined neocortical area/s. Our data, based on the detection of the main components of the preplate by double in situ hybridization, revealed that, in line with the classical inversion phenotype, preplate splitting does not occur in the caudal cortex, but it happens partially in rostral sections of the Reelin-deficient cortex. There, Ps/3-expressing cells (subplate cells) are mislocated in mid-upper cortical levels, indicating they have been pushed away by earlyborn cortical neurons.

Thalamocortical projections. Subplate cells participate in the guidance of the ingrowing thalamocortical efferents during development (Ghosh et al., 1990; Kanold and Luhmann, 2010). Thus, at E15.5, when thalamic axons reach the cortex, accumulate on subplate cells (Molnar and Blakemore, 1995). Afferent projections to the neocortex accompany the cellular ectopia in reeler (Caviness, 1976; Simmons et al., 1982). As axon guidance is only slightly affected in reeler (Harsan et al., 2013), the thalamic fiber pattern could tell us where subplate cells are. In reeler M1, despite few oblique afferents, the terminal fields of the thalamocortical axons target the area below the 
cortical plate, corresponding to the potential subplate zone. In reeler V1, however, fibers are obliquely oriented indicating that the target cells, the subplate cells, are abnormally located above the cortical plate. This data thus support the results from the preplate analysis.

Layer VI cells and the discrepancy in preplate splitting. One of the features often used to explain the layer inversion in reeler is that the first cohort of migrating projection neurons is unable to split the preplate. As a result, cortical layers V-II develop below (Caviness, 1982a; Gupta et al., 2002; Hoffarth et al., 1995; Sheppard and Pearlman, 1997). Moreover, since the preplate forms normally but LVI cells fail to split it in reeler, these cells are believed to be the first neurons that respond to Reelin. One interesting fact about these neurons is that, in contrast to future generations, they translocate directly into their final cortical location (Nadarajah et al., 2001a). The translocation implies the development of a long leading process that branches and anchors to the pia mater (Cooper, 2008), and having an impaired development of this process leads to cell location beneath the preplate (O'Dell et al., 2012; Sanada et al., 2004). Reelin is believed to regulate the directional dendritic growth into the marginal zone, rather than the position of LVI cells (Nichols and Olson, 2010; O'Dell et al., 2012). This project has shown that LVI cells pile up next to the pia mater in the inversion-like visual cortex, where there is an obvious superplate. Yet, those cells are split in a deep and superficial location, in the wild type-like motor cortex, where preplate splitting partially occurs. Thus, the results suggest that in the rostral cortex in reeler the first cohort of migrating projection neurons might preserve the somal translocation mode and successfully bypass older neurons (in this case, subplate cells), in contrast to caudal regions where translocation is likely to be prevented. Time-lapse imaging of E13.5- GFP in utero electroporated neurons from the rostral and caudal reeler cortex would be of great value to validate this statement. Similar to previous studies (O'Dell et al., 2012), the orientation of the cell body and primary neurites of this first cohort of migrating neurons upon reaching the marginal zone has to be also evaluated, in the rostral and caudal cortex of the mutant mouse. Future experiments could also consist of a simultaneous detection of thalamic fibers and subplate cells and the investigation of their contacts along the rostro-caudal axis of the reeler brain. 


\section{NEOCORTEX ASSEMBLY: birth dating analysis}

Layer development in the neocortex relies on appropriate cell production and positioning during embryonic and early postnatal stages (Gupta et al., 2002; Tan and Shi, 2013). Thus, those are certainly the processes to investigate when facing a phenotype that, like reeler, challenges cortical lamination. The sequential administration of thymidine analogs reveal that, in the absence of Reelin, cohorts of projection neurons are produced on schedule, and exit and migrate towards the cortical plate following the sequence by which neurons are generated. Neurogenesis, in general, does not seem to be affected either. The initial behavior of early- and late- born neurons in reeler mirrors the development of wild type mice. However, at P7, when migration is known to be finished, the reeler neocortex fails to constitute any structure similar to a layer and differences in cortical assembling over the rostro-caudal axis becomes clear. Additionally, the combination of the administration of BrdU and BrdU-derivatives with the Flash Tag labeling demonstrates that both direct and indirect neurogenesis from RGCs and IPs contribute to the distinct (mis)laminated patterns in reeler.

\section{Labeling techniques}

Novel cell birth dating approach. One of the techniques used to evaluate the assembly of the neocortex is the administration of thymidine analogs during development. Thymidine analogs are permanently incorporated into the DNA of the cells that divide by the time of injection, and their subsequent progeny (Cavanagh et al., 2011). Therefore, temporal and spatial arrangements of labeled cortical cells can be evaluated by the analysis of brain sections of animals that received the thymidine analog(s) at particular developmental stages. Major findings about neocortical formation and neurogenesis, as well as fundamental aspects of reeler and other mouse lines, were based on the administration of tritiated thymidine to rodents (Angevine and Sidman, 1961; Cavanagh et al., 2011; Caviness and Sidman, 1973; Hack et al., 2007b). Tritiated thymidine is no longer in use, and more up-to-date studies are based on single or double sequential administration of the halogenated thymidine analogs available (Azim et al., 2009; Tuttle et al., 2010; Vega and Peterson, 2005; Wiegreffe et al., 2015). However, for someone studying neocortical development, this approach implies that one has to sacrifice the tracing of a large number of cortical neurons that could not be labeled, compromising the conclusions. 
In this project, the neocortical histogenesis was followed over brain development by using a novel cell-birth dating approach, which combines three different types of thymidine analogs. The data proved that the sequential administration of EdU (E13.5), CldU (E14.5) and IdU (E15.5), ensures the labeling of infragranular, granular and supragranular cells in the developing cortex, respectively. Thus, the relative cell position of neurons fated for the three main cortical compartments can be simultaneously evaluated on the same brain section and over the entire process of cortical lamination (shown here at least until postnatal day 7). Nevertheless, if a particular cohort of projection neurons is of special interest for a given scientific project, EdU would be the best choice, as the chemical-based detection preserves the integrity of the labeled cells (Cavanagh et al., 2011). Additionally, based on a custom-written script and open-access bioinformatic tools (RStudio and Fiji), we could set a threshold that facilitates the discrimination of the first progeny of the progenitor pool. That progeny becomes postmitotic soon after the pulse and thus is strongly labeled by the halogenated thymidine analog injected by that time (Polleux et al., 1998; Rakic, 1973). In the present study, that progeny is preferentially restricted to certain layers, speaking in favor of the fact that neurons that have a common birth date are fated to the same layer, as previously reported (Angevine and Sidman, 1961; Gelman and Marin, 2010; Takahashi et al., 1999; Tissir and Goffinet, 2003)

Thymidine analogs vs Flash Tag. Thymidine analogs label the progeny of RGCs in the basal VZ but also IPs in the SVZ, obstructing a clear definition of the progenitor pool that has uptaken the reagent. Moreover, as the entire progeny of a given labeled progenitor retains the label, an unambiguous discrimination of a single cohort of cortical neurons is not possible per se. The Flash Tag labeling technique, on the contrary, labels isochronic crowds of only apical VZ-derived cells. Thus, this technique guarantees a pure classification of the progenitor pool that uptakes the dye and the labeling of a restricted cluster of newborn projection neurons, more suitable for fine tracing (Telley et al., 2016). Moreover, and reported here for the first time, basic morphological details of migrating neurons labeled by Flash Tag can be detected, even by examining the native signal. In combination with a thymidine analog chronic labeling, this technique also allows a clear discrimination of the apical VZ- progeny born by direct neurogenesis.

Notice that the administration of thymidine analogs and Flash Tag lead to the labeling of projection neurons, but also interneurons. These neurons are principally born in the subpallial telencephalon and migrate tangentially during embryonic development to populate the cortex (Marin and Rubenstein, 2001). Since this subject was not addressed in the present study, further 
experiments will need to evaluate the interneuron population in the injected animals and its contribution to the different cortical patterns in reeler.

\section{Lessons learnt from the analysis of neocortical histogenesis in Reelin-deficient mice.}

Although it has been shown that radial glial cells express Dab1, ApoER2 and VLDLR (Luque et al., 2003; Uchida et al., 2009), it is believed that Reelin influences the formation of the glial scaffold (Chai et al., 2015; Hartfuss et al., 2003; Weiss et al., 2003) , rather than the proliferative activity of the progenitor pool. In agreement with those studies, cell density analysis of early-and late- born neurons indicate that the number of cells born at a particular embryonic time in reeler does not differ from that seen in wild type littermates, neither in the rostral nor in the caudal neocortex. No significant differences were seen in the location or number of $\mathrm{Pax6}^{+}(\mathrm{RGC})$ or $\mathrm{Tbr2}^{+}$ (IP) progenitor cells either. Moreover, cells migrate according to the sequence they are born, as early-born neurons delaminate and travel beyond late-born neurons, when analyzed at late embryonic development, in both genotypes and all cortical regions. Altogether, the data indicates that in a Reelin-deficient environment, neurogenesis does not seem to be severely affected, cohorts of projection neurons are produced on schedule (as shown by (Caviness and Sidman, 1973; Polleux et al., 1998)) and the delamination from the ventricular zone follows the sequence by which neurons are generated. Thus, defects in the migration of neurons that have already left the germinal zone must be responsible for the cellular ectopia of the reeler cortex. Then, when exactly do the deficiencies start to appear?

Neocortical histogenesis at E17.5 and P7. Neurons have to travel a considerably long distance to get to their final destination. Based on the outline of the radial migration process (Gupta et al., 2002; Nadarajah and Parnavelas, 2002), it is possible to predict in which cortical compartment neurons born at a given time should be, if those neurons did not encounter complications on their way. Thereby, if analyzed at late stages of embryonic development, neurons that are born at early stages of corticogenesis (e.g. E13.5) are expected to be found in upper cortical levels, while neurons born later (e.g. E15.5) should be located in lower levels of the cortical column. Here it is shown that, in wild type, but also in reeler cortex, E13.5- born neurons have settled down in the cortical plate, and E15.5- born neurons reside in the intermediate zone at late stages of cortical development, in the anterior and posterior cortex. It suggests, as pointed out previously (Caviness and Rakic, 1978; Tissir and Goffinet, 2003), that the initial behavior of reeler neurons mirrors, in general, the development of wild type mice. However, at P7, when 
migration is known to be finished and all projection neurons are installed in the cortex in wild type animals, the mislamination in reeler is evident, as E13.5- $\left(\mathrm{EdU}^{+}\right), \mathrm{E} 14.5-\left(\mathrm{CldU}^{+}\right)$and E15.5$\left(\mathrm{IdU}^{+}\right)$born neurons fail to constitute any structure similar to a layer in all areas examined. Moreover, differences in the cortical assembly in anterior and posterior cortices in the mutant mouse became clear. In rostral areas, despite all cell spreading, E13.5-born neurons are preferentially located in superficial and deep regions of the cortex and E15.5-born cells have reached upper cortical levels. Quite the opposite can be found in caudal areas of reeler mice: E13.5-born cells are located in upper cortical regions whereas E15.5-born neurons are largely found below. The comparison of the location of early- and late- born neurons at E17.5 and P7, helped us to narrow down the critical aspects of corticogenesis that lead to the cortical lamination failure in reeler. In agreement with previous studies (Caviness and Rakic, 1978; Chai et al., 2015; Tissir and Goffinet, 2003), and validated in the present study by using a simultaneous pulse of EdU and Flash Tag, this critical window corresponds to the time point when neurons arrive at the cortical plate and have the mission to bypass older neurons.

LII/III cells reach upper layers in the Reelin-deficient neocortex. By combining the thymidine analog and the Flash Tag labeling approaches, it could be demonstrated that: 1.) the distinct rostral and caudal phenotypes seem to depend on both the IPs and RGCs progeny, born by direct or indirect neurogenesis, and 2.) SVZ/VZ- and VZ- derived layer II/III cells reach upper cortical levels in the rostral cortex of (P7) reeler mice. These results argue against one of the most famous conceptions about reeler. It is generally believed that cortical neurons in reeler are not capable to scope their final destination, and ectopically stop migrating in lower cortical layers (Caffrey et al., 2014; Chai et al., 2015; Gupta et al., 2002; Tabata and Nakajima, 2002). The latest studies that consolidated this statement are mainly based on the tracing of in utero E14.5electroporated neurons, which basically targets LIV cells, and its analysis up to P0, in reeler or phenotypically reeler-like rodents (e.g. scrambler or yotari) (Chai et al., 2015; Tabata and Nakajima, 2002). However, at PO radial migration is still an ongoing process. Thus, even though by this time LIV cells are migrating behind their wild type counterparts in a given cortex (not specified by the authors), it does not exclude the possibility that at least some of these cells might reach their designated location in the cortex a few days later. Interestingly, Rorb-neurons (LIV), although scattered, were restricted to mid-cortical levels in all areas examined, where they are expected to be. On the other hand, the results also verified that SVZ/VZ- and VZ-derived layer II/III cells are restricted to deep levels of the cortical column in caudal regions of the very same mice. 
Thus, the data indicates that the assumption that projection neurons stop migrating prematurely and are ectopically located in deep cortical regions in reeler, requires a reformulation to represent the complexity of the Reelin-deficient cortex. It might reflect neuronal migration and cortical lamination of the (V1) caudal cortex, but not of the (M1) rostral cortex.

\section{NEURONAL MIGRATION OF LATE-BORN NEURONS IN THE REELIN DEFICIENT NEOCORTEX}

Radially migrating neurons have a remarkably long journey to undertake before they differentiate in their defined cortical location. Layer $\mathrm{VI}$ and layer $\mathrm{V}$ to II/III migrate to the cortex differently. Thus, layer VI projection neurons (born at E13.5) migrate by somal translocation, while layer V, IV and II/III cells (born from approximately at E14.5-E16.5) predominantly migrate by glia-guided locomotion (Nadarajah and Parnavelas, 2002). As the data presented here was mainly focused on the migration of late-born neurons, only the migration of these cells will be further discussed.

The migration of layer II/III cells (and also layer IV and V cells) towards the cortex can be divided into five main phases. (1) Cells first depart from the germinal zone (VZ) in a bipolar shape. (2) Neurons are then arrested in a multipolar morphology in the multipolar accumulation zone (MAZ) in the SVZ for a day. During this time multipolar cells might also migrate to random directions or towards the top of the intermediate zone (known as multipolar migration, (Tabata and Nakajima, 2003)). (3) Projection neurons switch back to bipolar morphology and retrogradely migrate towards the VZ. (4) After contacting the apical surface of the VZ, neurons reverse polarity and migrate in the direction of the cortical plate by using the glial-scaffold (known as glia-guided migration or locomotion). (5) Near their final cortical destination, neurons bypass older neurons by terminal somal translocation (Nadarajah et al., 2001a; Noctor et al., 2004; Rakic, 1972a) (Fig.2). Neuronal migration routes during embryonic brain development are complex and require precise coordination (Marin et al., 2010). Neurons on their way to the cortex are subjected to the reorganization of the cytoskeleton, cellular organelles and cell-surface proteins (Ayala et al., 2007; Cooper, 2013). These events lead to morphological rearrangements and changes in polarity, which determine the direction of migration of a given neuron. Not surprising, Reelin is believed 
to be one the extracellular proteins that drive these and other key events in radial migration (Curran and D'Arcangelo, 1998; Frotscher and Zhao, 2010; Rice and Curran, 2001)

\section{Neuronal migration in the MAZ-IZ in the Reelin-deficient neocortex}

The examination of the first stages of neuronal migration of late-born neurons by in utero electroporation and Flash Tag labeling demonstrate that, in the absence of Reelin, cells become multipolar in the MAZ, adopt a bipolar morphology that maintains the front-rear polarity, and migrate towards the pial surface as a cohesive population.

Cells in the Multipolar Accumulation zone (MAZ). It is thought that Reelin influences migrating excitatory neurons even beneath the CP. After secretion, Reelin is subjected to enzymatic cleavage in vivo, producing different fragments of various length and diffusion properties. The full-length protein remains mainly next to the secretion source, in the marginal zone. On the contrary, the smaller fragments diffuse throughout the cortical wall, indicating its participation in processes away from the secretion site (Jossin et al., 2007; Lambert de Rouvroit et al., 1999). Consequently, Jossin and Cooper showed that Reelin activates Rap1 (Ras-related GTPase), which in turn regulates NCad (neural cadherin). NCad happens to be needed to orient multipolar cells towards the pial surface (Jossin and Cooper, 2011). Some other groups have confirmed that Reelin, but also ApoER2 (one of its main receptors), are expressed, at the mRNA and protein level, in the SVZ and IZ of the wild type brain (Hirota et al., 2014; Trommsdorff et al., 1999; Uchida et al., 2009). More precisely, ApoER2 is highly expressed in the multipolar cells in the MAZ. Thus, it has been proposed that Reelin-ApoER2 binding might regulate the formation and/or migration of the multipolar cells, by triggering a signaling cascade that modifies the dynamics of their processes (Hirota et al., 2014). In line with this hypothesis, our results suggest that the overexpression of ApoER2 in vivo might indeed promote a multipolar morphology, as many E14.5-electroporated neurons remained preferentially multipolar a day after transfection. However, one interesting finding indicates that the Reelin-signaling cascade does not seem to be crucial to induce multipolar morphology: GFP labeled multipolar cells were found in the MAZ, in rostral and caudal cortices, of Reelin-deficient mice. Interestingly, Britto and colleagues proved that the migration speed of multipolar E14.5 GFP-labeled cells in the IZ is not influenced by the absence of Reelin. Multipolar cells show, however, greater meandering behavior on their way to the cortex (Britto et al., 2011). Highly branched and dynamic processes are thought to be needed for route finding (Cooper, 2013). Thus, it seems fair to speculate that, in the absence of Reelin, an 
increase in the tangential movement of multipolar cells might be needed to sense the concentration of chemoattractants and repellents in the surrounding, in order to instruct the correct direction of migration. This hypothesis could be also applicable for those quasi-bipolar cells (more than one process) found in the $I Z$ of the caudal visual cortex, as well as for the disoriented neurons in the cortex in the absence of Reelin. It has been also suggested that the multipolar stage and the multipolar migration may play a role in the establishment of a preferential connectivity in clonally related neurons. These neurons are also thought to travel together (Li et al., 2012; Yu et al., 2009). Interestingly, the Flash Tag labeling showed that lateborn and early-born neurons travel as a cohesive population through the $\mathrm{IZ}$ in rostral and caudal cortices in wild type, but also, in Reelin-deficient animals.

Bipolar migrating neurons. Multipolar neurons transition back into bipolar neurons. One of the multiple and unstable processes, characteristic of multipolar cells, is stabilized while the others retract (Cooper, 2013). Bipolar cells are highly polarized, and show a thick leading process at the front and a thin trailing process emerging at the rear (Rakic, 1972a). The centrosome and the Golgi apparatus moves to a dilation in the basal end of the leading neurite. The nucleus follows the centrosome, being then relocated into the dominant process. The dominant or leading process directs then the migration of the neuron (Bellion et al., 2005; Cooper, 2013). Bipolar neurons migrate to upper cortical levels by locomotion, with the leading process partially entwined around a glial fiber (Nadarajah et al., 2001a; Rakic, 1972a).

Reelin has been proposed as a polarizing cue by several groups (Jossin and Cooper, 2011; Matsuki et al., 2010; Meseke et al., 2013). However, its importance in that function is debatable. Here it was shown that in the Reelin-deficient cortex, E15.5-electroporated neurons in the IZ keep the front-rear polarity typical of bipolar migrating neurons, in rostral and caudal neocortical sections. Remarkably, the leading process maintains a pia-orientation in reeler. And, even though Reelin has been proved to orientate the Golgi towards the leading neurite (Matsuki et al., 2010; Meseke et al., 2013), our experiments showed that in the absence of Reelin, the Golgi (detected by GM130 immunostaining) is located in the basal portion of the leading neurite, as in wild type animals. The centrosome and the Golgi (together with recycling endosomes) in front of the nucleus provides microtubules and protein complexes to the front of the cell, needed for moving forward, and regulates nuclear translocation (Li and Gundersen, 2008; Nadarajah et al., 2001a; Tsai and Gleeson, 2005). Thus, the data indicate that neurons in the absence of Reelin preserve their polarity and the capacity to migrate forward towards the cortical plate. 
Bipolar morphology and pia-oriented cell polarity in the absence of Reelin. If there is no Reelin, what could be responsible for the bipolar morphology and the pia-oriented cell polarity? Glial fibers could be one of the candidates. Glial fibers are the substrates that projection neurons use to reach upper cortical levels. Thus, the bipolar morphology of neurons in reeler might be conferred by the interaction with the glial scaffold. The gap-junction subunit Connexin43 (Cx43) is believed to play a major role in the glia-guided neuronal migration, as it serves as an adhesive contact between the migrating neurons and the glial fibers. Interestingly, Cx43 has been also linked to the stabilization of the leading process (Elias et al., 2007). Despite its importance in gliacell interaction, the levels of expression of $\mathrm{C} \times 43$ in reeler have not been evaluated yet. Polarizing Reelin-independent machinery should be also considered. Par3 and aPKC form a polarity complex involved in the polarization of numerous cell types. It has been suggested that Par3 may activate Rap1, triggering cytoskeleton rearrangements that lead to the establishment of the leading process (Chen and Macara, 2005). Par3 binds to ApoER2 (Pasten et al., 2015), and ApoER2 is expressed in reeler, at the mRNA (unpublished data from the group) and protein level (Hirota et al., 2014), in the MAZ and the $I Z$.

Whether the glial scaffold and $\mathrm{Cx} 43$ are indeed responsible for the pia-oriented cell polarity in reeler has to be investigated in future experiments. For instance, the level of expression could be evaluated by immunostaining and western blot. Whether Par3 interacts with ApoER2 and influences cell polarity in the absence of Reelin also has to be validated. The interaction of Par3ApoER2 in reeler could be corroborated in the future by a pull-down assay of sorted multipolarGFP labeled cells (by in utero electroporation) from reeler pups or by FRET-microscopy. Additionally, an elegant polarity-assay in vivo could consist of an in utero electroporation with GFP and a centrosome-marker-expressing plasmid enabling its tracing in living cells from reeler mice by time-lapse imaging.

\section{Neuronal migration in the cortical plate/cortex in Reelin-deficient mice}

The examination of late-born neurons at late stages of migration indicates that, in the absence of Reelin, most of the neurons in the IZ-CP border have a pia-directed radial process, with the cisGolgi situated basally, suggesting that, as in wild type animals, they are heading towards the outer brain surface. Neurons in reeler, however, lose the capacity to migrate as a spatially-coherent cohort while they cross the cortex. At the end of the first postnatal week, when radial migration is completed, neurons display a highly sparse distribution in the rostral and caudal reeler 
neocortex. Only neurons in rostral sections manage to conquer upper cortical levels and (some) stabilize their apical dendrites towards the brain surface. In caudal sections, these cells are restricted to lower cortical layers and extend their apical dendrite towards variable and unpredictable directions.

Bipolar neurons in the IZ-CP border. Late-born cortical projection neurons reach the upper level of the IZ preferentially by glia-guided locomotion. However, some neurons might use the multipolar migration mode (Tabata and Nakajima, 2003). It has been proposed that the SP plays a role in the multipolar to bipolar transition of those cells. Maruyama and colleagues observed that cells were multipolar or bipolar with more than one neurite below the SP zone, but had a thick unbranched leading process after bypassing the SP cells (Ohtaka-Maruyama and Okado, 2015). Thus, they suggested that cells may receive a cue to transition into a neat bipolar morphology from the SP zone. In the present study, it was shown that in the reeler brain preplate splitting partially takes place in rostral cortices, but does not occur in caudal regions. Nevertheless, migrating neurons close to the intermediate zone are not multipolar, neither in rostral, nor in caudal cortices of the developing reeler brain. Instead, E15.5-born neurons have what appears to be a neat leading process that invades the cortical plate, indicating that the SP zone is not essential to induce a bipolar morphology. Whether these bipolar cells display additional thin neurites has to be further investigated by higher-resolution microscopy and singleneuron reconstructions.

Direction of migration in the absence of Reelin. Reelin has been proposed to act as an attractive signal, as principal neurons of all laminated structures of the brain migrate towards the Reelin-rich marginal zone (D'Arcangelo, 2005; D'Arcangelo et al., 1995; Frotscher and Zhao, 2010). Interestingly, this study has shown that late-born migrating neurons in Reelin-deficient animals preserve the direction of movement towards the marginal zone, even upon entering the cortex, in agreement with previous observations (Chai et al., 2015). Notably, migration towards the marginal zone is presumably preserved along the rostro-caudal axis, as neurons in rostral and caudal regions maintain a pialward orientation of their migratory trajectory. But even though these cells are supposed to migrate by using the glial scaffold and even assuming that those fibers might impose a bipolar morphology on them, their migration towards the end-feet of the glial fibers cannot be explained simply by a glia-cell interaction. Neurons direct their migration in response to extracellular chemoattractant signals (Cooper, 2013). In the absence of Reelin, Semaphorin-3A (Sema-3A) appears to be an appropriate replacement for that function. Sema-3A 
is an extracellular diffusible factor expressed in a descending gradient throughout the cortex, and it has been shown that even multipolar projection neurons express receptors for this protein. By manipulating the expression of Sema-3A it was demonstrated that it is essential for the radial migration of layer II/III neurons, as it is serves as chemoattractant for them (Chen et al., 2008). Thus, Sema-3A might be the chemoattractant signal that stimulates neurons to migrate towards the pia matter, from the multipolar stage until reaching the cortical plate, in the Reelin-deficient neocortex. To validate this hypothesis, the level of expression of Sema-3A and Sema-3A receptors in migrating neurons in reeler must be investigated. The potential to induce neuronal migration towards a Sema-3A rich zone in those neurons, can be evaluated, for instance, by in vitro slice cell culture assays.

Migrating neurons in the cortical plate. The absence of Reelin did not prevent multipolar shape induction, multipolar to bipolar transition, cell polarity or pia-oriented migration of layer II/III neurons, neither in rostral, nor in caudal regions of the developing neocortex in reeler (Fig.34). However, when radial migration is finished, late-born neurons are located in the upper cortex in rostral sections, but restricted to the lower cortex in caudal regions. How can this be explained?

Neurons in reeler lose the capacity to migrate as a spatially-coherent cohort, as they do below the cortical plate, while they navigate through cortex. Although it has been suggested that cell dispersion is due to the absence of Reelin (Dekimoto et al., 2010; Kubo et al., 2010), the lack of cell aggregation happens to be similar in rostral and caudal cortical areas in reeler. Thus, it does not explain the differences seen in these areas. One alternative factor that could explain the rostro-caudal differences is the transverse neurogenic gradient (Suter et al., 2007). The rostralto-caudal neurogenic and morphogenic gradient might lead to variations in neuronal migration. Consequently, neurons in rostral cortices might enter the cortical plate before their fellows located more caudally. Therefore, they have the chance to travel further, occupying upper cortical levels. However, this hypothesis can be discarded due to two facts: a) neurons in wild type animals are subjected to the same gradient and reach the layer II/III zone without any difficulty, and b) even though it could be considered that layer II/III fated cells are located in lower cortical levels due to time limitations, layer IV and V cells were given enough time to reach their designated cortical destination and they did not do so either.

On the other hand, one could hypothesize that neurons in caudal regions might suffer a significant reduction on their migratory speed upon reaching cortex, hence their ectopic location 
in the lower cortex. Neurons use radial glial fibers as guides to reach their destination and, interestingly, radial fibers are altered in reeler (Hartfuss et al., 2003; Hunter-Schaedle, 1997). Moreover, neurons in reeler are believed to be abnormally associated with the radial fibers (Pinto Lord and Caviness, 1979). The glial scaffold seems to be more affected in caudal regions, as fibers show greater meandering on their way to the pia mater. Thus, particularly abnormal glial fibers might have led to a reduction in the locomotion speed in caudal regions of the Reelin-deficient neocortex, contributing to their ectopic location. Future experiments could assess areadependent glia-neuron interaction by electron microscopy or live STED-microscopy. Net speed and trajectories of glia-guided neurons over the rostro-caudal axis of the reeler brain could be evaluated by time-lapse imaging.

Glia-guided migration ends when the leading process of migrating neurons reaches the marginal zone. Cells then detach from the glial scaffold and bypass its predecessors by gliaindependent somal translocation (called terminal translocation). To do so, neurons anchor their leading process to the marginal zone and pull the soma towards its tip (Nadarajah et al., 2001a; Sekine et al., 2012). Terminal translocation has been demonstrated to be dependent on Reelin (Ballif et al., 2004; Franco et al., 2011; Gil-Sanz et al., 2013; Olson et al., 2006; Sekine et al., 2012). Even though layer II/III cells migrate much greater distances using the glial scaffold, somal translocation, a phylogenetically conserved mode of migration, is thought to be essential to bypass older neurons in the cortical plate (Hirota and Nakajima, 2017; Meyer et al., 1998; Nadarajah et al., 2001a; Sekine et al., 2011). Thus, differences in the capacity of migrating past their predecessors by somal translocation could be also account for the final location of late-born neurons in the rostral and caudal cortex in the absence of Reelin. Time lapse imaging of E15.5 GFP-labeled neurons from rostral and caudal neocortical sections of the reeler brain at several time points during development (e.g. E19-P2 and P5-P8) will be needed to validate this hypothesis.

\section{Cell fate and early dendritogenesis}

Cell fate. The reeler phenotype is particularly interesting because it indicates that the fate of the majority of neurons is determined by their time and site of origin and not by their ultimate place of differentiation in the brain (Cooper, 2008). Previous studies have shown that the progenitor pool is fate-restricted to produce lower- or upper- layer neurons as corticogenesis proceeds (Chen et al., 2005; Franco et al., 2012; Nieto et al., 2004). It has been also demonstrated 
that by the time a neuron becomes postmitotic, it has the instructions to migrate to the layer it is destined to and develops its birth-date fate even when being ectopically located (Desai and McConnell, 2000; McConnell and Kaznowski, 1991). Recent studies suggested, on the contrary, that over-dosage of certain genes induce neurons to change their destined position in the cortex, which then adopt the fate of the host layer (Katayama et al., 2017). Here it has been shown that FT+ E15.5-born neurons (fated to LII/III) remain "fateless" in reeler even at P7, when migration is known to be completed and neurons start to differentiate. These cells did not show immunoreactivity for Ctip2 (a marker for infragranular layers) when located in lower cortical levels. Moreover, regardless of their location in the cortical column, these cells remained also largely Satb2-negative (a maker for granular and supragranular layers). However, due to the low number of samples, one must be cautious. Further experiments are surely required (e.g. robust quantifications, additional cell fate markers, older animals) to investigate whether cells remain truly molecularly "fateless" and, if this is the case, when those neurons get their final identity and what this will be.

Immature neurons. It was also observed that although differentiated cells extend their processes in various directions (as shown in previous studies (Chai et al., 2015; Landrieu and Goffinet, 1981; Tabata and Nakajima, 2002), some rostral neurons are able to extend the apical dendrite towards the brain surface, similar to the wild type cortex. After a bipolar cell has completed migration, the thick leading process is thought to become the apical dendrite, and the axon emerges from the thin trailing process at the rear (Hatanaka and Murakami, 2002; Rakic, 1982; Schwartz et al., 1991). Whether this effect is due to an area-dependent premature loss of polarity, a defect in glia interaction (Elias et al., 2007), or distinct distribution of the axon rich strata (Pinto Lord and Caviness, 1979), has to be investigated in the future.

\section{M1 vs V1: SUMMARY AND HYPOTHETICAL MODEL}

The layer-fate marker approach shows that each area has a unique degree of cell intermingling, indicating that cortical areas should be evaluated separately. Interestingly, within the areadependent (mis)laminated patterns, the primary motor cortex (M1) and the primary visual cortex (V1) differs the most in the layout of their delamination. Thus, the rostrally-located M1 cortex shows a neuronal distribution more comparable to wild type, while the caudally-located V1 cortex 
is shaped in a pattern more similar to an inversion. The present study has shown that LVI cells are presumably able to split the preplate in the rostral motor cortex, while they fail to do so in the caudal visual cortex. Our data also indicate that in the absence of Reelin, cells become multipolar, transition back to the bipolar morphology typical of a migrating neuron, maintain a front-rear polarity and migrate towards the marginal zone, in both areas, mirroring the wild type situation (Fig.34). However, LII/III neurons populate the upper cortex in rostral sections, but not in caudal regions.

It is worth to mention that the final location of an excitatory neuron in the neocortex is determined not only by the journey accomplished by that particular neuron, but also by the migratory behavior of neurons born afterwards (Gupta et al., 2002). Moreover, and as Caviness denoted, the degree of mixing of projection neurons in a particular cortical column indicates the capacity to which neurons penetrate the zone occupied by their predecessors (Caviness, 1982a); and projection neurons enter that zone by somal translocation. Interestingly, LVI cells migrate by somal translocation, and even though LV to LIV cells migrate preferentially glia-guided, they require this mode of migration as a last step to bypass their predecessors (Nadarajah and Parnavelas, 2002).

Thus, and although highly speculative, it seems conceivable to hypothesize that, the inside-out (or quasi- wild type) pattern in $\mathrm{M} 1$ and the outside-in (or quasi- inverted) pattern in V1, could be the consequence of the preservation (M1) or restriction (V1) of the somal translocation mode by migrating neurons (Fig.34). This hypothesis has to be validated in the future, for instance, by time-lapse imaging of labeled neurons from reeler at different times of development and destined to different cortical regions. However, even if this hypothesis happens to be correct, it does not explain why in the absence of Reelin cortical lamination in rostral sections seems to mirror the normal neocortical development, while the visual cortex shows the classical inversion phenotype. It would be exciting to uncover the gene-expression profiles of projection neurons located in different cortical regions of the Reelin-deficient mice, by for instance RNA-sequencing of sorted Flash-Tag labeled cells. Expression profiling of secretable proteins that might take over Reelin's role in an area-dependent fashion would be also of great interest. 
wild type

reeler

A

B rostral (M1)

C rostral (V1)

CX

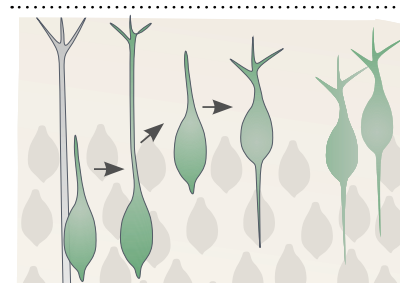

iz

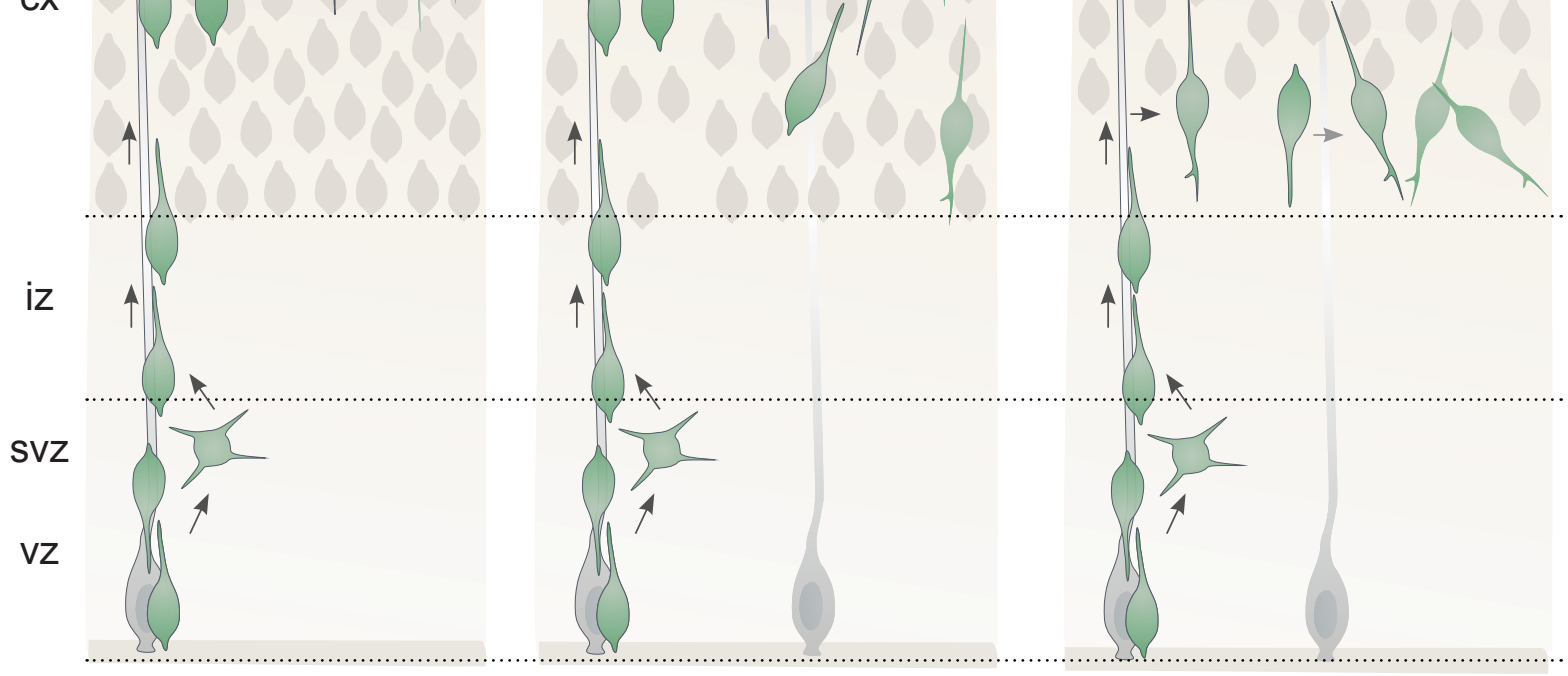

SVZ
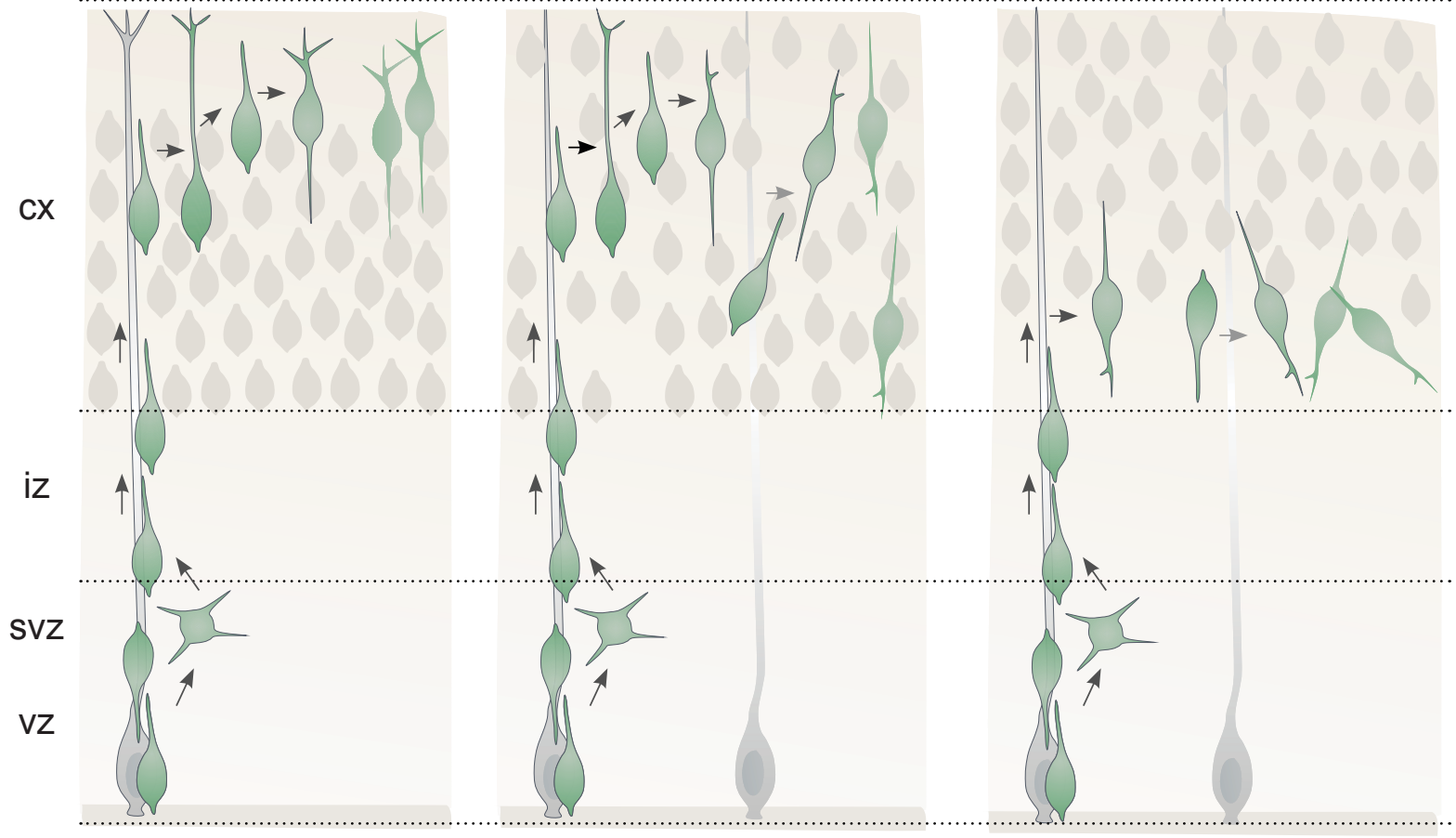

Figure 34. Radial migration of late-born neurons. Neuronal migration of projection neurons in wild type animals (A) and hypothetical models of neuronal migration in the primary motor cortex $(M 1, B)$ and the primary visual cortex (V1, C) in the reeler brain. Bipolar neurons exit the ventricular zone (VZ), and accumulate in a multipolar morphology in the multipolar accumulation zone of the subventricular zone (SVZ). Neurons may exhibit a retrograde migration towards the apical surface of the VZ (not shown). Bipolar neurons then reverse polarity and migrate by locomotion (or glia-guided migration) to reach upper cortical levels. Final cortical location is achieved by somal translocation (Nadarajah et al., 2001a). Migrating neurons in rostral (M1) or caudal (V1) cortical regions of Reelin-deficient mice became multipolar and accumulate in the MAZ. Cells also adopt a bipolar shape and migrate, presumably by using the glia scaffold, towards the pial surface. Upon reaching the cortex, projection neurons of the rostral neocortex reach upper cortical levels, and likely bypass older neurons by somal translocation. Differentiated neurons project the apical dendrite upwards but also to various directions. In contrast, neurons in the caudal neocortex stop migrating at lower cortical levels and are presumably unable to bypass preformed layers. Differentiated neurons extend apical dendrites preferentially downwards or to capricious directions. Abbreviations: vz, ventricular zone; svz, subventricular zone; iz, intermediate zone; cx, cortex. 


\section{REELIN AND THE REELER CORTEX New findings, yet more questions}

Reelin and its contribution to brain development has kept neurobiologist busy and captivated for years. Several roles have been proposed for this multifaceted protein during corticogenesis. Thus, it is considered as (i) a polarizing factor (Jossin and Cooper, 2011), (ii) attractant cue (Caviness and Rakic, 1978; Jossin et al., 2004; Magdaleno et al., 2002; Olson et al., 2006; Sanada et al., 2004; Wang et al., 2018), (iii) stop and detachment signal (Dulabon et al., 2000; Feng et al., 2007; Frotscher, 1997a; Hack et al., 2002; Super et al., 2000), and (iv) a cytoskeleton stabilizer at various levels (Assadi et al., 2003; Chai et al., 2015; Chai et al., 2009; Franco et al., 2011; Luque et al., 2003; Sekine et al., 2012). Some of these results influenced the "detach and go" and "detach and stop" models by which Reelin is supposed to instruct inside-out cortical lamination (for a review see, (Cooper, 2008)). However, the accuracy of Reelin's soles are still debatable. Our data indicates that neuronal polarity and pia-oriented migration is preserved in the absence of Reelin. The results also suggest that Reelin might be of crucial importance when neurons navigate through the neocortex, especially by the time they undergo terminal translocation.

The unique area-dependent cortical patterns and migration defects in reeler might be the combination of the absence of one or several of Reelin' s roles. However, it is quite surprising that there is some sort of order under the apparent chaos. If lamination and migration in Reelindeficient mice are dictated by a still-unknown molecular mechanisms and whether they act in an area-dependent fashion has be to be investigated in future projects.

Reelin might be in charge of all of the roles it has been assigned to. But for these to be understood, the cortex is not allowed to be considered as a uniform structure. Cell-type or areadependent secondary effects due its absence have to be taken into consideration as well. Furthermore, novel ligands for Reelin and Reelin receptors are waiting to be discovered. 


\section{SUMMARY}

The adult mammalian neocortex is divided into six vertical layers, as well as into numerous specialized tangential areas that are defined by distinct cytoarchitectures and specific wiring patterns. Precise neocortical function depends on the accurate development of these layers and areas. The formation of layers relies, on an appropriate cell production, migration and positioning during embryonic and early postnatal stages. The large extracellular matrix glycoprotein Reelin, encoded by the reelin (Reln) gene (D'Arcangelo et al., 1999) and secreted by Cajal-Reztius cells in the marginal zone of the developing neocortex, plays a key role in orchestrating these events. The reeler mouse strain (Falconer, 1951), a mouse with a homozygous loss of function of the reelin gene, played a fundamental role in the process of understanding normal brain development and directed the hypothesis about Reelin function. However, diverse models of Reelin action, influenced by the incomplete description of the reeler phenotype, have been proposed and still remain controversial.

On the basis of layer-specific mRNA expression we could demonstrate that the reeler cortex, usually described as "inverted", is disrupted in a more complex and, highly areadependent manner. These cortical lamination patterns range from an intermingled, but more similar to the wild type phenotype in the rostral motor cortex (quasi-wild type), via an almost layer- disappearance in the intermediate somatosensory cortex, to a more inverted phenotype in the caudal visual cortex (quasi-inverted). Developmentally, the distribution of early- and lateborn neurons (establishing infra- vs. supragranular layers in wild type) are largely non-consistent across cortical regions in reeler. These differences create an inside-out to an outside-in lamination gradient, through the anterior-posterior axis in of the reeler cortex. In line with these data, the expression of preplate-specific genes and trajectories of thalamic fibers during development, indicate that preplate splitting is altered, but generally takes place in rostral cortical areas, while it is completely absent in the caudal cortex. Neocortical neurogenesis and neuronal migration was examined in Reelin-deficient mice, making use of in utero electroporation, FlashTag labeling and a novel nucleoside analog cell birth-dating techniques. We could demonstrate that in the absence for Reelin, neurogenesis seems to be unaffected and that direct as well as indirect VZ- and SVZborn progeny contribute to the cellular intermingling. We also could show that, at early stages of migration, cells migrate as a cohesive population, and neuronal morphology, cell polarity and radial migration is preserved in the rostral motor cortex as well as the caudal visual cortex in 
reeler. However, the various reeler phenotypes become apparent at the moment in which neurons enter the cortical plate. Despite an equivalent cell scattering, only late-born neurons in the rostral cortex are able to reach upper cortical levels and direct, at least partially, their dendrites upwards. In contrast, late-born neurons in the caudal cortex ectopically stop migrating and differentiate closer to the white matter, extending its processes towards various and unpredictable directions.

The data from the present study demonstrate that the neocortex should not be considered generally and highlights the complexity of the Reelin-deficient neocortex. Our results also indicate that cell-type an area-dependent secondary effects due to the absence of Reelin should be taken into account for the evaluation of the reeler neocortex. 


\section{REFERENCES}

Aikawa, H., Nonaka, I., Woo, M., Tsugane, T., and Esaki, K. (1988). Shaking rat Kawasaki (SRK): a new neurological mutant rat in the Wistar strain. Acta neuropathologica 76, 366-372.

Alcántara, S., Ruiz, M., D'Arcangelo, G., Ezan, F., de Lecea, L., Curran, T., Sotelo, C., and Soriano, E. (1998). Regional and cellular patterns of reelin mRNA expression in the forebrain of the developing and adult mouse. J Neurosci 18, 7779-7799.

Andersen, O.M., Benhayon, D., Curran, T., and Willnow, T.E. (2003). Differential binding of ligands to the apolipoprotein E receptor 2. Biochemistry 42, 9355-9364.

Angevine, J.B., Jr., and Sidman, R.L. (1961). Autoradiographic study of cell migration during histogenesis of cerebral cortex in the mouse. Nature 192, 766-768.

Arlotta, P., Molyneaux, B.J., Chen, J., Inoue, J., Kominami, R., and Macklis, J.D. (2005). Neuronal subtype-specific genes that control corticospinal motor neuron development in vivo. Neuron 45, 207-221.

Assadi, A.H., Zhang, G., Beffert, U., McNeil, R.S., Renfro, A.L., Niu, S., Quattrocchi, C.C., Antalffy, B.A., Sheldon, M., Armstrong, D.D., et al. (2003). Interaction of reelin signaling and Lis1 in brain development. Nat Genet 35, 270-276.

Ayala, R., Shu, T., and Tsai, L.H. (2007). Trekking across the brain: the journey of neuronal migration. Cell 128, 29-43.

Azim, E., Jabaudon, D., Fame, R.M., and Macklis, J.D. (2009). SOX6 controls dorsal progenitor identity and interneuron diversity during neocortical development. Nat Neurosci 12, 1238-1247.

Badea, A., Nicholls, P.J., Johnson, G.A., and Wetsel, W.C. (2007). Neuroanatomical phenotypes in the reeler mouse. Neuroimage 34, -

Ballif, B.A., Arnaud, L., Arthur, W.T., Guris, D., Imamoto, A., and Cooper, J.A. (2004). Activation of a Dab1/CrkL/C3G/Rap1 pathway in Reelin-stimulated neurons. Curr Biol 14, 606-610.

Bayer, S.A., and Altman, J. (1990). Development of layer I and the subplate in the rat neocortex. Exp Neurol 107, 48-62.

Beffert, U., Morfini, G., Bock, H.H., Reyna, H., Brady, S.T., and Herz, J. (2002). Reelin-mediated signaling locally regulates protein kinase B/Akt and glycogen synthase kinase 3 beta. J Biol Chem 277, 49958-49964.

Bellion, A., Baudoin, J.P., Alvarez, C., Bornens, M., and Metin, C. (2005). Nucleokinesis in tangentially migrating neurons comprises two alternating phases: forward migration of the Golgi/centrosome associated with centrosome splitting and myosin contraction at the rear. J Neurosci 25, 5691-5699.

Benhayon, D., Magdaleno, S., and Curran, T. (2003). Binding of purified Reelin to ApoER2 and VLDLR mediates tyrosine phosphorylation of Disabled-1. Brain research Molecular brain research 112, 33-45.

Boyle, M.P., Bernard, A., Thompson, C.L., Ng, L., Boe, A., Mortrud, M., Hawrylycz, M.J., Jones, A.R., Hevner, R.F., and Lein, E.S. (2011). Cell-type-specific consequences of Reelin deficiency in the mouse neocortex, hippocampus, and amygdala. The Journal of Comparative Neurology 519, 2061-2089.

Breunig, J.J., Silbereis, J., Vaccarino, F.M., Sestan, N., and Rakic, P. (2007). Notch regulates cell fate and dendrite morphology of newborn neurons in the postnatal dentate gyrus. Proc Natl Acad Sci U S A 104, 20558-20563. 
Britanova, O., de Juan Romero, C., Cheung, A., Kwan, K.Y., Schwark, M., Gyorgy, A., Vogel, T., Akopov, S., Mitkovski, M., Agoston, D., et al. (2008). Satb2 is a postmitotic determinant for upper-layer neuron specification in the neocortex. Neuron 57, 378-392.

Britto, J.M., Tait, K.J., Johnston, L.A., Hammond, V.E., Kalloniatis, M., and Tan, S.S. (2011). Altered speeds and trajectories of neurons migrating in the ventricular and subventricular zones of the reeler neocortex. Cereb Cortex 21, 1018-1027.

Bystron, I., Blakemore, C., and Rakic, P. (2008). Development of the human cerebral cortex: Boulder Committee revisited. Nat Rev Neurosci 9, 110-122.

Caffrey, J.R., Hughes, B.D., Britto, J.M., and Landman, K.A. (2014). An in silico agent-based model demonstrates Reelin function in directing lamination of neurons during cortical development. PLoS One 9, e110415.

Cajal, S. (1861). Sur la structure de lecorce cerebrale de quelques mammiferes. Cellule 7, 123-176.

Catalano, S.M., and Shatz, C.J. (1998). Activity-dependent cortical target selection by thalamic axons. Science 281, 559-562.

Cavanagh, B.L., Walker, T., Norazit, A., and Meedeniya, A.C. (2011). Thymidine analogues for tracking DNA synthesis. Molecules (Basel, Switzerland) 16, 7980-7993.

Caviness, V.S. (1976). Patterns of cell and fiber distribution in the neocortex of the reeler mutant mouse. J Comp Neurol 170, 435-447.

Caviness, V.S. (1982a). Neocortical histogenesis in normal and reeler mice- a developmentalstudy based upon [H-3] thymidin autoradiography. Dev Brain Res 4, 293-302.

Caviness, V.S., Jr. (1982b). Development of neocortical afferent systems: studies in the reeler mouse. Neurosci Res Program Bull 20, 560-569.

Caviness, V.S., Jr., and Sidman, R.L. (1973). Time of origin or corresponding cell classes in the cerebral cortex of normal and reeler mutant mice: an autoradiographic analysis. J Comp Neurol 148, 141-151.

Caviness, V.S., and Rakic, P. (1978). Mechanisms of cortical development: a view from mutations in mice. Annu Rev Neurosci 1, -.

Chai, X., Fan, L., Shao, H., Lu, X., Zhang, W., Li, J., Wang, J., Chen, S., Frotscher, M., and Zhao, S. (2015). Reelin Induces Branching of Neurons and Radial Glial Cells during Corticogenesis. Cereb Cortex 25, 3640-3653.

Chai, X., Förster, E., Zhao, S., Bock, H.H., and Frotscher, M. (2009). Reelin acts as a stop signal for radially migrating neurons by inducing phosphorylation of $n$-cofilin at the leading edge. Commun Integr Biol 2, -.

Chai, X., and Frotscher, M. (2016). How does Reelin signaling regulate the neuronal cytoskeleton during migration? Neurogenesis 3, e1242455.

Chen, B., Schaevitz, L.R., and McConnell, S.K. (2005). Fezl regulates the differentiation and axon targeting of layer 5 subcortical projection neurons in cerebral cortex. Proc Natl Acad Sci U S A 102, 17184-17189.

Chen, G., Sima, J., Jin, M., Wang, K.Y., Xue, X.J., Zheng, W., Ding, Y.Q., and Yuan, X.B. (2008). Semaphorin-3A guides radial migration of cortical neurons during development. Nat Neurosci 11, 36-44.

Chen, X., and Macara, I.G. (2005). Par-3 controls tight junction assembly through the Rac exchange factor Tiam1. Nature cell biology 7, 262-269. 
Chou, S.J., Babot, Z., Leingartner, A., Studer, M., Nakagawa, Y., and O'Leary, D.D. (2013). Geniculocortical input drives genetic distinctions between primary and higher-order visual areas. Science 340, 1239-1242.

Chowdhury, T.G., Jimenez, J.C., Bomar, J.M., Cruz-Martin, A., Cantle, J.P., and Portera-Cailliau, C. (2010). Fate of cajal-retzius neurons in the postnatal mouse neocortex. Front Neuroanat 4, 10.

Cooper, J.A. (2008). A mechanism for inside-out lamination in the neocortex. Trends Neurosci 31, 113-119.

Cooper, J.A. (2013). Cell biology in neuroscience: mechanisms of cell migration in the nervous system. The Journal of cell biology 202, 725-734.

Curran, T., and D'Arcangelo, G. (1998). Role of reelin in the control of brain development. Brain Res Brain Res Rev 26, -.

D'Arcangelo, G. (2005). The reeler mouse: anatomy of a mutant. Int Rev Neurobiol 71, 383-417.

D'Arcangelo, G. (2014). Reelin in the Years: Controlling Neuronal Migration and Maturation in the Mammalian Brain. Advances in Neuroscience 2014, 19.

D'Arcangelo, G., and Curran, T. (1998). Reeler: new tales on an old mutant mouse. BioEssays : news and reviews in molecular, cellular and developmental biology 20, 235-244.

D'Arcangelo, G., Homayouni, R., Keshvara, L., Rice, D.S., Sheldon, M., and Curran, T. (1999). Reelin is a ligand for lipoprotein receptors. Neuron 24, 471-479.

D'Arcangelo, G., Miao, G.G., Chen, S.C., Soares, H.D., Morgan, J.I., and Curran, T. (1995). A protein related to extracellular matrix proteins deleted in the mouse mutant reeler. Nature $374,719-$ 723.

D'Arcangelo, G., Nakajima, K., Miyata, T., Ogawa, M., Mikoshiba, K., and Curran, T. (1997). Reelin is a secreted glycoprotein recognized by the CR-50 monoclonal antibody. J Neurosci 17, 23-31.

Dekimoto, H., Terashima, T., and Katsuyama, Y. (2010). Dispersion of the neurons expressing layer specific markers in the reeler brain. Development, Growth \& Differentiation 52, 181-193.

del Rio, J.A., Martinez, A., Fonseca, M., Auladell, C., and Soriano, E. (1995). Glutamate-like immunoreactivity and fate of Cajal-Retzius cells in the murine cortex as identified with calretinin antibody. Cereb Cortex 5, 13-21.

Derer, P. (1979). Evidence for the occurrence of early modifications in the 'glia limitans' layer of the neocortex of the reeler mutant mouse. Neurosci Lett 13, 195-202.

Derer, P., and Derer, M. (1990). Cajal-Retzius cell ontogenesis and death in mouse brain visualized with horseradish peroxidase and electron microscopy. Neuroscience 36, 839-856.

Desai, A.R., and McConnell, S.K. (2000). Progressive restriction in fate potential by neural progenitors during cerebral cortical development. Development 127, 2863-2872.

Dulabon, L., Olson, E.C., Taglienti, M.G., Eisenhuth, S., McGrath, B., Walsh, C.A., Kreidberg, J.A., and Anton, E.S. (2000). Reelin binds alpha3beta1 integrin and inhibits neuronal migration. Neuron 27, 33-44.

Elias, L.A., Wang, D.D., and Kriegstein, A.R. (2007). Gap junction adhesion is necessary for radial migration in the neocortex. Nature 448, 901-907.

Falconer, D.S. (1951). Two new mutants, 'trembler' and 'reeler', with neurological actions in the house mouse (Mus musculus L.). J Genet 50, 192-201.

Feng, L., Allen, N.S., Simo, S., and Cooper, J.A. (2007). Cullin 5 regulates Dab1 protein levels and neuron positioning during cortical development. Genes Dev 21, 2717-2730. 
Ferrer, I., Bernet, E., Soriano, E., del Rio, T., and Fonseca, M. (1990). Naturally occurring cell death in the cerebral cortex of the rat and removal of dead cells by transitory phagocytes. Neuroscience 39, 451-458.

Ferrere, A., Vitalis, T., Gingras, H., Gaspar, P., and Cases, O. (2006). Expression of Cux-1 and Cux2 in the developing somatosensory cortex of normal and barrel-defective mice. The anatomical record Part A, Discoveries in molecular, cellular, and evolutionary biology 288, 158-165.

Folsom, T.D., and Fatemi, S.H. (2013). The involvement of Reelin in neurodevelopmental disorders. Neuropharmacology 68, 122-135.

Forster, E., Tielsch, A., Saum, B., Weiss, K.H., Johanssen, C., Graus-Porta, D., Muller, U., and Frotscher, M. (2002). Reelin, Disabled 1, and beta 1 integrins are required for the formation of the radial glial scaffold in the hippocampus. Proc Natl Acad Sci U S A 99, 13178-13183.

Franco, S.J., Gil-Sanz, C., Martinez-Garay, I., Espinosa, A., Harkins-Perry, S.R., Ramos, C., and Muller, U. (2012). Fate-restricted neural progenitors in the mammalian cerebral cortex. Science 337, 746-749.

Franco, S.J., Martinez-Garay, I., Gil-Sanz, C., Harkins-Perry, S.R., and Muller, U. (2011). Reelin regulates cadherin function via Dab1/Rap1 to control neuronal migration and lamination in the neocortex. Neuron 69, 482-497.

Frotscher, M. (1997a). Dual role of Cajal-Retzius cells and reelin in cortical development. Cell Tissue Res 290, 315-322.

Frotscher, M., and Zhao, S.T. (2010). Go or Stop? Divergent Roles of Reelin in Radial Neuronal Migration. Neuroscientist 16, 421-434.

Gao, P., Postiglione, M.P., Krieger, T.G., Hernandez, L., Wang, C., Han, Z., Streicher, C., Papusheva, E., Insolera, R., Chugh, K., et al. (2014). Deterministic progenitor behavior and unitary production of neurons in the neocortex. Cell 159, 775-788.

Garcia-Moreno, F., Lopez-Mascaraque, L., and De Carlos, J.A. (2007). Origins and migratory routes of murine Cajal-Retzius cells. J Comp Neurol 500, 419-432.

Garey, L.J. (1999). Brodmann's Localisation in the Cerebral Cortex (Translated from German). Imperial College Press.

Gatter, K.C., Sloper, J.J., and Powell, T.P. (1978). The intrinsic connections of the cortex of area 4 of the monkey. Brain : a journal of neurology 101, 513-541.

Gelman, D.M., and Marin, O. (2010). Generation of interneuron diversity in the mouse cerebral cortex. Eur J Neurosci 31, 2136-2141.

Ghosh, A., Antonini, A., McConnell, S.K., and Shatz, C.J. (1990). Requirement for subplate neurons in the formation of thalamocortical connections. Nature 347, 179-181.

Gil-Sanz, C., Franco, S.J., Martinez-Garay, I., Espinosa, A., Harkins-Perry, S., and Muller, U. (2013). Cajal-Retzius cells instruct neuronal migration by coincidence signaling between secreted and contact-dependent guidance cues. Neuron 79, 461-477.

Goffinet, A.M. (1979). An early development defect in the cerebral cortex of the reeler mouse. A morphological study leading to a hypothesis concerning the action of the mutant gene. Anat Embryol (Berl) 157, 205-216.

Goffinet, A.M., and Lyon, G. (1979). Early histogenesis in the mouse cerebral cortex: a Golgi study. Neurosci Lett 14, 61-66.

Gong, S., Zheng, C., Doughty, M.L., Losos, K., Didkovsky, N., Schambra, U.B., Nowak, N.J., Joyner, A., Leblanc, G., Hatten, M.E., et al. (2003). A gene expression atlas of the central nervous system based on bacterial artificial chromosomes. Nature 425, 917-925. 
Gotthardt, M., Trommsdorff, M., Nevitt, M.F., Shelton, J., Richardson, J.A., Stockinger, W., Nimpf, J., and Herz, J. (2000). Interactions of the low density lipoprotein receptor gene family with cytosolic adaptor and scaffold proteins suggest diverse biological functions in cellular communication and signal transduction. J Biol Chem 275, 25616-25624.

Gotz, M., and Huttner, W.B. (2005). The cell biology of neurogenesis. Nature reviews Molecular cell biology $6,777-788$.

Graus-Porta, D., Blaess, S., Senften, M., Littlewood-Evans, A., Damsky, C., Huang, Z., Orban, P., Klein, R., Schittny, J.C., and Muller, U. (2001). Beta1-class integrins regulate the development of laminae and folia in the cerebral and cerebellar cortex. Neuron 31, 367-379.

Gupta, A., Tsai, L.H., and Wynshaw-Boris, A. (2002). Life is a journey: a genetic look at neocortical development. Nat Rev Genet 3, 342-355.

Guy, J., Sachkova, A., Mock, M., Witte, M., Wagener, R.J., and Staiger, J.F. (2017). Intracortical Network Effects Preserve Thalamocortical Input Efficacy in a Cortex Without Layers. Cereb Cortex 27, 4851-4866.

Guy, J., and Staiger, J.F. (2017). The Functioning of a Cortex without Layers. Front Neuroanat 11, 54.

Hack, I., Bancila, M., Loulier, K., Carroll, P., and Cremer, H. (2002). Reelin is a detachment signal in tangential chain-migration during postnatal neurogenesis. Nat Neurosci 5, 939-945.

Hack, I., Hellwig, S., Junghans, D., Brunne, B., Bock, H.H., Zhao, S., and Frotscher, M. (2007b). Divergent roles of ApoER2 and Vldlr in the migration of cortical neurons. Development 134, 3883-3891.

Hanashima, C., Fernandes, M., Hebert, J.M., and Fishell, G. (2007). The role of Foxg1 and dorsal midline signaling in the generation of Cajal-Retzius subtypes. J Neurosci 27, 11103-11111.

Harsan, L.A., David, C., Reisert, M., Schnell, S., Hennig, J., von Elverfeldt, D., and Staiger, J.F. (2013). Mapping remodeling of thalamocortical projections in the living reeler mouse brain by diffusion tractography. Proc Natl Acad Sci U S A 110, E17.597-1806.

Hartfuss, E., Forster, E., Bock, H.H., Hack, M.A., Leprince, P., Luque, J.M., Herz, J., Frotscher, M., and Gotz, M. (2003). Reelin signaling directly affects radial glia morphology and biochemical maturation. Development 130, 4597-4609.

Hatanaka, Y., and Murakami, F. (2002). In vitro analysis of the origin, migratory behavior, and maturation of cortical pyramidal cells. J Comp Neurol 454, 1-14.

Hevner, R., Daza, R.A., Rubenstein, J.L., Stunnenberg, H., Olavarria, J., and Englund, C. (2003a). Beyond laminar fate: toward a molecular classification of cortical projection/pyramidal neurons. Dev Neurosci 25, 139-151.

Hevner, R.F., Shi, L., Justice, N., Hsueh, Y., Sheng, M., Smiga, S., Bulfone, A., Goffinet, A.M., Campagnoni, A.T., and Rubenstein, J.L. (2001). Tbr1 regulates differentiation of the preplate and layer 6. Neuron 29, 353-366.

Hiesberger, T., Trommsdorff, M., Howell, B.W., Goffinet, A., Mumby, M.C., Cooper, J.A., and Herz, J. (1999). Direct binding of Reelin to VLDL receptor and ApoE receptor 2 induces tyrosine phosphorylation of disabled-1 and modulates tau phosphorylation. Neuron 24, 481-489.

Hirota, Y., Kubo, K.I., Katayama, K.I., Honda, T., Fujino, T., Yamamoto, T.T., and Nakajima, K. (2014). Reelin receptors ApoER2 and VLDLR are expressed in distinct spatio-temporal patterns in developing mouse cerebral cortex. J Comp Neurol.

Hirota, Y., and Nakajima, K. (2017). Control of Neuronal Migration and Aggregation by Reelin Signaling in the Developing Cerebral Cortex. Frontiers in cell and developmental biology 5, 40. 
Hoerder-Suabedissen, A., and Molnar, Z. (2015). Development, evolution and pathology of neocortical subplate neurons. Nat Rev Neurosci 16, 133-146.

Hoffarth, R.M., Johnston, J.G., Krushel, L.A., and van der Kooy, D. (1995). The mouse mutation reeler causes increased adhesion within a subpopulation of early postmitotic cortical neurons. J Neurosci 15, 4838-4850.

Horton, A.C., Racz, B., Monson, E.E., Lin, A.L., Weinberg, R.J., and Ehlers, M.D. (2005). Polarized secretory trafficking directs cargo for asymmetric dendrite growth and morphogenesis. Neuron 48, 757-771.

Howell, B.W., Herrick, T.M., and Cooper, J.A. (1999). Reelin-induced tyrosine [corrected] phosphorylation of disabled 1 during neuronal positioning. Genes Dev 13, 643-648.

Hunter-Schaedle, K.E. (1997). Radial glial cell development and transformation are disturbed in reeler forebrain. J Neurobiol 33, 459-472.

Ikeda, Y., and Terashima, T. (1997). Expression of reelin, the gene responsible for the reeler mutation, in embryonic development and adulthood in the mouse. Dev Dyn 210, -

Inoue, K., Terashima, T., Nishikawa, T., and Takumi, T. (2004). Fez1 is layer-specifically expressed in the adult mouse neocortex. Eur J Neurosci 20, 2909-2916.

Jiang, X., and Nardelli, J. (2016). Cellular and molecular introduction to brain development. Neurobiology of disease 92, 3-17.

Jossin, Y., and Cooper, J.A. (2011). Reelin, Rap1 and N-cadherin orient the migration of multipolar neurons in the developing neocortex. Nat Neurosci 14, 697-703.

Jossin, Y., Gui, L., and Goffinet, A.M. (2007). Processing of Reelin by embryonic neurons is important for function in tissue but not in dissociated cultured neurons. J Neurosci 27, 42434252.

Jossin, Y., Ignatova, N., Hiesberger, T., Herz, J., Lambert de Rouvroit, C., and Goffinet, A.M. (2004). The central fragment of Reelin, generated by proteolytic processing in vivo, is critical to its function during cortical plate development. J Neurosci 24, 514-521.

Kandel, E.R., Schwartz, J.M., Jessel, T.M., Siegelbaum, S.A., and Hudspeth, A.J. (2013). Principles of Neural Science (McGraw Hill).

Kanold, P.O., and Luhmann, H.J. (2010). The subplate and early cortical circuits. Annu Rev Neurosci 33, 23-48.

Kanold, P.O., and Shatz, C.J. (2006). Subplate neurons regulate maturation of cortical inhibition and outcome of ocular dominance plasticity. Neuron 51, 627-638.

Katayama, K.I., Hayashi, K., Inoue, S., Sakaguchi, K., and Nakajima, K. (2017). Enhanced expression of Pafah1b1 causes over-migration of cerebral cortical neurons into the marginal zone. Brain Struct Funct 222, 4283-4291.

Kim, D.H., lijima, H., Goto, K., Sakai, J., Ishii, H., Kim, H.J., Suzuki, H., Kondo, H., Saeki, S., and Yamamoto, T. (1996). Human apolipoprotein E receptor 2. A novel lipoprotein receptor of the low density lipoprotein receptor family predominantly expressed in brain. J Biol Chem 271, 8373-8380.

Komuro, H., and Rakic, P. (1995). Dynamics of granule cell migration: a confocal microscopic study in acute cerebellar slice preparations. J Neurosci 15, 1110-1120.

Kostovic, I., and Rakic, P. (1980). Cytology and time of origin of interstitial neurons in the white matter in infant and adult human and monkey telencephalon. J Neurocytol 9, 219-242. 
Kubo, K., Honda, T., Tomita, K., Sekine, K., Ishii, K., Uto, A., Kobayashi, K., Tabata, H., and Nakajima, K. (2010). Ectopic Reelin induces neuronal aggregation with a normal birthdate-dependent "inside-out" alignment in the developing neocortex. J Neurosci 30, 10953-10966.

Kwan, K.Y., Sestan, N., and Anton, E.S. (2012). Transcriptional co-regulation of neuronal migration and laminar identity in the neocortex. Development 139, 1535-1546.

Lambert de Rouvroit, C., de Bergeyck, V., Cortvrindt, C., Bar, I., Eeckhout, Y., and Goffinet, A.M. (1999). Reelin, the extracellular matrix protein deficient in reeler mutant mice, is processed by a metalloproteinase. Exp Neurol 156, 214-217.

Landrieu, P., and Goffinet, A. (1981). Inverted pyramidal neurons and their axons in the neocortex of reeler mutant mice. Cell Tissue Res 218, 293-301.

Leone, D.P., Srinivasan, K., Chen, B., Alcamo, E., and McConnell, S.K. (2008). The determination of projection neuron identity in the developing cerebral cortex. Curr Opin Neurobiol 18, 28-35.

Li, R., and Gundersen, G.G. (2008). Beyond polymer polarity: how the cytoskeleton builds a polarized cell. Nature reviews Molecular cell biology 9, 860-873.

Li, Y., Lu, H., Cheng, P.L., Ge, S., Xu, H., Shi, S.H., and Dan, Y. (2012). Clonally related visual cortical neurons show similar stimulus feature selectivity. Nature 486, 118-121.

Liu, Q., Dwyer, N.D., and O'Leary, D.D. (2000). Differential expression of COUP-TFI, CHL1, and two novel genes in developing neocortex identified by differential display PCR. J Neurosci 20, 76827690 .

Luque, J.M., Morante-Oria, J., and Fairen, A. (2003). Localization of ApoER2, VLDLR and Dab1 in radial glia: groundwork for a new model of reelin action during cortical development. Brain Res Dev Brain Res 140, 195-203.

Magdaleno, S., Keshvara, L., and Curran, T. (2002). Rescue of ataxia and preplate splitting by ectopic expression of Reelin in reeler mice. Neuron 33, 573-586.

Mariani, J., Crepel, F., Mikoshiba, K., Changeux, J.P., and Sotelo, C. (1977). Anatomical, physiological and biochemical studies of the cerebellum from Reeler mutant mouse. Philosophical transactions of the Royal Society of London Series B, Biological sciences 281, 1-28.

Marin-Padilla, M. (1978). Dual origin of the mammalian neocortex and evolution of the cortical plate. Anat Embryol (Berl) 152, 109-126.

Marin, O., and Rubenstein, J.L. (2001). A long, remarkable journey: tangential migration in the telencephalon. Nat Rev Neurosci 2, 780-790.

Marin, O., Valiente, M., Ge, X., and Tsai, L.H. (2010). Guiding neuronal cell migrations. Cold Spring Harb Perspect Biol 2, a001834.

Marx, M., Qi, G., Hanganu-Opatz, I.L., Kilb, W., Luhmann, H.J., and Feldmeyer, D. (2017). Neocortical Layer 6B as a Remnant of the Subplate - A Morphological Comparison. Cereb Cortex 27, 1011-1026.

Matsuki, T., Matthews, R.T., Cooper, J.A., van der Brug, M.P., Cookson, M.R., Hardy, J.A., Olson, E.C., and Howell, B.W. (2010). Reelin and stk25 have opposing roles in neuronal polarization and dendritic Golgi deployment. Cell 143, 826-836.

McConnell, S.K., Ghosh, A., and Shatz, C.J. (1989). Subplate neurons pioneer the first axon pathway from the cerebral cortex. Science 245, 978-982.

McConnell, S.K., and Kaznowski, C.E. (1991). Cell cycle dependence of laminar determination in developing neocortex. Science 254, 282-285.

Meseke, M., Cavus, E., and Forster, E. (2013). Reelin promotes microtubule dynamics in processes of developing neurons. Histochem Cell Biol 139, 283-297. 
Meyer, G. (1987). Forms and spatial arrangement of neurons in the primary motor cortex of man. J Comp Neurol 262, 402-428.

Meyer, G. (2010). Building a human cortex: the evolutionary differentiation of Cajal-Retzius cells and the cortical hem. J Anat 217, 334-343.

Meyer, G., Perez-Garcia, C.G., Abraham, H., and Caput, D. (2002). Expression of p73 and Reelin in the developing human cortex. J Neurosci 22, 4973-4986.

Meyer, G., Soria, J.M., Martinez-Galan, J.R., Martin-Clemente, B., and Fairen, A. (1998). Different origins and developmental histories of transient neurons in the marginal zone of the fetal and neonatal rat cortex. J Comp Neurol 397, 493-518.

Miyata, T., Kawaguchi, A., Okano, H., and Ogawa, M. (2001). Asymmetric inheritance of radial glial fibers by cortical neurons. Neuron 31, 727-741.

Miyata, T., Kawaguchi, A., Saito, K., Kawano, M., Muto, T., and Ogawa, M. (2004). Asymmetric production of surface-dividing and non-surface-dividing cortical progenitor cells. Development 131, 3133-3145.

Miyata, T., Nakajima, K., Aruga, J., Takahashi, S., Ikenaka, K., Mikoshiba, K., and Ogawa, M. (1996). Distribution of a reeler gene-related antigen in the developing cerebellum: an immunohistochemical study with an allogeneic antibody CR-50 on normal and reeler mice. J Comp Neurol 372, 215-228.

Molnar, Z., and Blakemore, C. (1995). How do thalamic axons find their way to the cortex? Trends Neurosci 18, 389-397.

Molyneaux, B.J., Arlotta, P., Menezes, J.R., and Macklis, J.D. (2007). Neuronal subtype specification in the cerebral cortex. Nat Rev Neurosci 8, 427-437.

Montiel, J.F., Wang, W.Z., Oeschger, F.M., Hoerder-Suabedissen, A., Tung, W.L., Garcia-Moreno, F., Holm, I.E., Villalon, A., and Molnar, Z. (2011). Hypothesis on the dual origin of the Mammalian subplate. Front Neuroanat 5, 25.

Morest, D.K. (1970). A study of neurogenesis in the forebrain of opossum pouch young. Zeitschrift fur Anatomie und Entwicklungsgeschichte 130, 265-305.

Nadarajah, B., Brunstrom, J.E., Grutzendler, J., Wong, R.O., and Pearlman, A.L. (2001a). Two modes of radial migration in early development of the cerebral cortex. Nature Neuroscience 4, 143-150.

Nadarajah, B., and Parnavelas, J.G. (2002). Modes of neuronal migration in the developing cerebral cortex. Nat Rev Neurosci 3, 423-432.

Nakamura, N., Rabouille, C., Watson, R., Nilsson, T., Hui, N., Slusarewicz, P., Kreis, T.E., and Warren, G. (1995). Characterization of a cis-Golgi matrix protein, GM130. The Journal of cell biology 131, 1715-1726.

Nakayama, D., Baraki, Z., Onoue, K., Ikegaya, Y., Matsuki, N., and Nomura, H. (2015). Frontal association cortex is engaged in stimulus integration during associative learning. Curr Biol 25, 117-123.

Nichols, A.J., and Olson, E.C. (2010). Reelin promotes neuronal orientation and dendritogenesis during preplate splitting. Cereb Cortex 20, 2213-2223.

Nieto, M., Monuki, E.S., Tang, H., Imitola, J., Haubst, N., Khoury, S.J., Cunningham, J., Gotz, M., and Walsh, C.A. (2004). Expression of Cux-1 and Cux-2 in the subventricular zone and upper layers II-IV of the cerebral cortex. J Comp Neurol 479, 168-180.

Niwa, H., Yamamura, K., and Miyazaki, J. (1991). Efficient selection for high-expression transfectants with a novel eukaryotic vector. Gene 108, 193-199. 
Noctor, S.C., Flint, A.C., Weissman, T.A., Dammerman, R.S., and Kriegstein, A.R. (2001). Neurons derived from radial glial cells establish radial units in neocortex. Nature 409, 714-720.

Noctor, S.C., Martinez-Cerdeno, V., Ivic, L., and Kriegstein, A.R. (2004). Cortical neurons arise in symmetric and asymmetric division zones and migrate through specific phases. Nat Neurosci 7 , 136-144.

O'Dell, R.S., Ustine, C.J., Cameron, D.A., Lawless, S.M., Williams, R.M., Zipfel, W.R., and Olson, E.C. (2012). Layer 6 cortical neurons require Reelin-Dab1 signaling for cellular orientation, Golgi deployment, and directed neurite growth into the marginal zone. Neural Dev 7, 25.

Oeschger, F.M., Wang, W.Z., Lee, S., Garcia-Moreno, F., Goffinet, A.M., Arbones, M.L., Rakic, S., and Molnar, Z. (2012). Gene expression analysis of the embryonic subplate. Cereb Cortex 22, 1343-1359.

Ogawa, M., Miyata, T., Nakajima, K., Yagyu, K., Seike, M., Ikenaka, K., Yamamoto, H., and Mikoshiba, K. (1995). The reeler gene-associated antigen on Cajal-Retzius neurons is a crucial molecule for laminar organization of cortical neurons. Neuron 14, 899-912.

Ohtaka-Maruyama, C., and Okado, H. (2015). Molecular Pathways Underlying Projection Neuron Production and Migration during Cerebral Cortical Development. Frontiers in neuroscience 9, 447.

Okada, T., Keino-Masu, K., and Masu, M. (2007). Migration and nucleogenesis of mouse precerebellar neurons visualized by in utero electroporation of a green fluorescent protein gene. Neurosci Res 57, 40-49.

Olson, E.C., Kim, S., and Walsh, C.A. (2006). Impaired neuronal positioning and dendritogenesis in the neocortex after cell-autonomous Dab1 suppression. J Neurosci 26, 1767-1775.

Pasten, C., Cerda, J., Jausoro, I., Court, F.A., Caceres, A., and Marzolo, M.P. (2015). ApoER2 and Reelin are expressed in regenerating peripheral nerve and regulate Schwann cell migration by activating the Rac1 GEF protein, Tiam1. Mol Cell Neurosci 69, 1-11.

Paxinos, G., and Franklin, K. (2004). Mouse Brain in Stereotaxic Coordinates (Elsevier).

Paxinos, G., Koutcherov, Y., Wang, H., Halliday, G.M., and Watson, C. (2011). Atlas of the Developing Mouse Brain (Elseiver).

Pedraza, M., Hoerder-Suabedissen, A., Albert-Maestro, M.A., Molnar, Z., and De Carlos, J.A. (2014). Extracortical origin of some murine subplate cell populations. Proc Natl Acad Sci U S A $111,8613-8618$.

Pielecka-Fortuna, J., Wagener, R.J., Martens, A.K., Goetze, B., Schmidt, K.F., Staiger, J.F., and Lowel, S. (2014). The disorganized visual cortex in reelin-deficient mice is functional and allows for enhanced plasticity. Brain Struct Funct.

Pinto Lord, M.C., and Caviness, V.S., Jr. (1979). Determinants of cell shape and orientation: a comparative Golgi analysis of cell-axon interrelationships in the developing neocortex of normal and reeler mice. J Comp Neurol 187, 49-69.

Pohlkamp, T., David, C., Cauli, B., Gallopin, T., Bouche, E., Karagiannis, A., May, P., Herz, J., Frotscher, M., Staiger, J.F., et al. (2014). Characterization and distribution of Reelin-positive interneuron subtypes in the rat barrel cortex. Cereb Cortex 24, 3046-3058.

Polleux, F., Dehay, C., and Kennedy, H. (1998). Neurogenesis and commitment of corticospinal neurons in reeler. J Neurosci 18, 9910-9923.

Popovitchenko, T., and Rasin, M.R. (2017). Transcriptional and Post-Transcriptional Mechanisms of the Development of Neocortical Lamination. Front Neuroanat 11, 102.

Quah, B.J.C., and Parish, C.R. (2010). The Use of Carboxyfluorescein Diacetate Succinimidyl Ester (CFSE) to Monitor Lymphocyte Proliferation. Journal of Visualized Experiments : JoVE, 2259. 
Rakic, P. (1972a). Mode of cell migration to the superficial layers of fetal monkey neocortex. Journal of Comparative Neurology 145, 61-83.

Rakic, P. (1973). Kinetics of proliferation and latency between final cell division and onset of differentiation of cerebellar stellate and basket neurons. J Comp Neurol 147, 523-546.

Rakic, P. (1974). Neurons in rhesus monkey visual cortex: systematic relation between time of origin and eventual disposition. Science 183, 425-427.

Rakic, P. (1982). Early developmental events: cell lineages, acquisition of neuronal positions, and areal and laminar development. Neurosci Res Program Bull 20, 439-451.

Ramon y Cajal, S. (1991). New ideas on the structure of the nervous system in man and vertebrates. Journal of cognitive neuroscience 3, 300-301.

Retzius, G. (1893). Die Cajal'schen Zellen der Grosshirnrinde beim Menschen und bei Säugetieren. Biologische Untersuchungen 5, 1-8.

Rice, D.S., and Curran, T. (2001). Role of the reelin signaling pathway in central nervous system development. Annu Rev Neurosci 24, 1005-1039.

Saito, T. (2006). In vivo electroporation in the embryonic mouse central nervous system. Nature protocols 1, 1552-1558.

Saito, T., and Nakatsuji, N. (2001). Efficient gene transfer into the embryonic mouse brain using in vivo electroporation. Dev Biol 240, 237-246.

Sanada, K., Gupta, A., and Tsai, L.H. (2004). Disabled-1-regulated adhesion of migrating neurons to radial glial fiber contributes to neuronal positioning during early corticogenesis. Neuron 42 , 197-211.

Sauer, M.E., and Walker, B.E. (1959). Radioautographic study of interkinetic nuclear migration in the neural tube. Proceedings of the Society for Experimental Biology and Medicine Society for Experimental Biology and Medicine (New York, NY) 101, 557-560.

Schwartz, M.L., Rakic, P., and Goldman-Rakic, P.S. (1991). Early phenotype expression of cortical neurons: evidence that a subclass of migrating neurons have callosal axons. Proc Natl Acad Sci U S A 88, 1354-1358.

Sekine, K., Honda, T., Kawauchi, T., Kubo, K., and Nakajima, K. (2011). The outermost region of the developing cortical plate is crucial for both the switch of the radial migration mode and the Dab1-dependent "inside-out" lamination in the neocortex. J Neurosci 31, 9426-9439.

Sekine, K., Kawauchi, T., Kubo, K., Honda, T., Herz, J., Hattori, M., Kinashi, T., and Nakajima, K. (2012). Reelin controls neuronal positioning by promoting cell-matrix adhesion via inside-out activation of integrin alpha5beta1. Neuron 76, 353-369.

Senturk, A., Pfennig, S., Weiss, A., Burk, K., and Acker-Palmer, A. (2011). Ephrin Bs are essential components of the Reelin pathway to regulate neuronal migration. Nature 472, 356-360.

Senzaki, K., Ogawa, M., and Yagi, T. (1999). Proteins of the CNR family are multiple receptors for Reelin. Cell 99, 635-647.

Shen, Q., Wang, Y., Dimos, J.T., Fasano, C.A., Phoenix, T.N., Lemischka, I.R., Ivanova, N.B., Stifani, S., Morrisey, E.E., and Temple, S. (2006). The timing of cortical neurogenesis is encoded within lineages of individual progenitor cells. Nat Neurosci 9, 743-751.

Sheppard, A.M., and Pearlman, A.L. (1997). Abnormal reorganization of preplate neurons and their associated extracellular matrix: an early manifestation of altered neocortical development in the reeler mutant mouse. J Comp Neurol 378, 173-179.

Silva, L.R., Gutnick, M.J., and Connors, B.W. (1991). Laminar distribution of neuronal membrane properties in neocortex of normal and reeler mouse. J Neurophysiol 66, 2034-2040. 
Simmons, P.A., Lemmon, V., and Pearlman, A.L. (1982). Afferent and efferent connections of the striate and extrastriate visual cortex of the normal and reeler mouse. J Comp Neurol 211, 295308.

Skoglund, T.S., Pascher, R., and Berthold, C.H. (1997). The existence of a layer IV in the rat motor cortex. Cereb Cortex 7, 178-180.

Sugino, K., Hempel, C.M., Miller, M.N., Hattox, A.M., Shapiro, P., Wu, C., Huang, Z.J., and Nelson, S.B. (2006). Molecular taxonomy of major neuronal classes in the adult mouse forebrain. Nat Neurosci 9, 99-107.

Super, H., Del Rio, J.A., Martinez, A., Perez-Sust, P., and Soriano, E. (2000). Disruption of neuronal migration and radial glia in the developing cerebral cortex following ablation of Cajal-Retzius cells. Cereb Cortex 10, 602-613.

Suter, B., Nowakowski, R.S., Bhide, P.G., and Caviness, V.S. (2007). Navigating neocortical neurogenesis and neuronal specification: a positional information system encoded by neurogenetic gradients. J Neurosci 27, 10777-10784.

Tabata, H., and Nakajima, K. (2002). Neurons tend to stop migration and differentiate along the cortical internal plexiform zones in the Reelin signal-deficient mice. Journal of neuroscience research 69, 723-730.

Tabata, H., and Nakajima, K. (2003). Multipolar migration: the third mode of radial neuronal migration in the developing cerebral cortex. J Neurosci 23, 9996-10001.

Takahashi, T., Goto, T., Miyama, S., Nowakowski, R.S., and Caviness, V.S., Jr. (1999). Sequence of neuron origin and neocortical laminar fate: relation to cell cycle of origin in the developing murine cerebral wall. J Neurosci 19, 10357-10371.

Takahashi, T., Nowakowski, R.S., and Caviness, V.S., Jr. (1995). The cell cycle of the pseudostratified ventricular epithelium of the embryonic murine cerebral wall. J Neurosci 15, 6046-6057.

Tan, X., and Shi, S.H. (2013). Neocortical neurogenesis and neuronal migration. Wiley Interdiscip Rev Dev Biol 2, 443-459.

Telley, L., Govindan, S., Prados, J., Stevant, I., Nef, S., Dermitzakis, E., Dayer, A., and Jabaudon, D. (2016). Sequential transcriptional waves direct the differentiation of newborn neurons in the mouse neocortex. Science.

Terashima, T., Inoue, K., Inoue, Y., Mikoshiba, K., and Tsukada, Y. (1983). Distribution and morphology of corticospinal tract neurons in reeler mouse cortex by the retrograde HRP method. J Comp Neurol 218, 314-326.

Terashima, T., Inoue, K., Inoue, Y., Mikoshiba, K., and Tsukada, Y. (1985). Distribution and morphology of callosal commissural neurons within the motor cortex of normal and reeler mice. J Comp Neurol 232, 83-98.

Teta, M., Rankin, M.M., Long, S.Y., Stein, G.M., and Kushner, J.A. (2007). Growth and regeneration of adult beta cells does not involve specialized progenitors. Developmental cell 12, 817-826.

Tissir, F., and Goffinet, A.M. (2003). Reelin and brain development. Nat Rev Neurosci 4, 496-505.

Tissir, F., Lambert de Rouvroit, C., and Goffinet, A.M. (2002). The role of reelin in the development and evolution of the cerebral cortex. Braz J Med Biol Res 35, 1473-1484.

Tissir, F., Ravni, A., Achouri, Y., Riethmacher, D., Meyer, G., and Goffinet, A.M. (2009). DeltaNp73 regulates neuronal survival in vivo. Proc Natl Acad Sci U S A 106, 16871-16876.

Trommsdorff, M., Gotthardt, M., Hiesberger, T., Shelton, J., Stockinger, W., Nimpf, J., Hammer, R.E., Richardson, J.A., and Herz, J. (1999). Reeler/Disabled-like disruption of neuronal migration in knockout mice lacking the VLDL receptor and ApoE receptor 2. Cell 97, 689-701. 
Tsai, L.H., and Gleeson, J.G. (2005). Nucleokinesis in neuronal migration. Neuron 46, 383-388.

Tuttle, A.H., Rankin, M.M., Teta, M., Sartori, D.J., Stein, G.M., Kim, G.J., Virgilio, C., Granger, A., Zhou, D., Long, S.H., et al. (2010). Immunofluorescent detection of two thymidine analogues (CldU and IdU) in primary tissue. J Vis Exp.

Uchida, T., Baba, A., Perez-Martinez, F.J., Hibi, T., Miyata, T., Luque, J.M., Nakajima, K., and Hattori, M. (2009). Downregulation of functional Reelin receptors in projection neurons implies that primary Reelin action occurs at early/premigratory stages. J Neurosci 29, 10653-10662.

Vega, C.J., and Peterson, D.A. (2005). Stem cell proliferative history in tissue revealed by temporal halogenated thymidine analog discrimination. Nat Methods 2, 167-169.

Visel, A., Thaller, C., and Eichele, G. (2004). GenePaint.org: an atlas of gene expression patterns in the mouse embryo. Nucleic acids research 32, D552-556.

Wagener, R.J., David, C., Zhao, S.T., Haas, C.A., and Staiger, J.F. (2010). The Somatosensory Cortex of reeler Mutant Mice Shows Absent Layering But Intact Formation and Behavioral Activation of Columnar Somatotopic Maps. J Neurosci 30, 15700-15709.

Wagener, R.J., Witte, M., Guy, J., Mingo-Moreno, N., Kugler, S., and Staiger, J.F. (2016). Thalamocortical Connections Drive Intracortical Activation of Functional Columns in the Mislaminated Reeler Somatosensory Cortex. Cereb Cortex 26, 820-837.

Wang, S., Brunne, B., Zhao, S., Chai, X., Li, J., Lau, J., Failla, A.V., Zobiak, B., Sibbe, M., Westbrook, G.L., et al. (2018). Trajectory Analysis Unveils Reelin's Role in the Directed Migration of Granule Cells in the Dentate Gyrus. J Neurosci 38, 137-148.

Wang, W.Z., Hoerder-Suabedissen, A., Oeschger, F.M., Bayatti, N., Ip, B.K., Lindsay, S., Supramaniam, V., Srinivasan, L., Rutherford, M., Mollgard, K., et al. (2010). Subplate in the developing cortex of mouse and human. J Anat 217, 368-380.

Weiss, K.H., Johanssen, C., Tielsch, A., Herz, J., Deller, T., Frotscher, M., and Forster, E. (2003). Malformation of the radial glial scaffold in the dentate gyrus of reeler mice, scrambler mice, and ApoER2/VLDLR-deficient mice. J Comp Neurol 460, 56-65.

Wichterle, H., Garcia-Verdugo, J.M., and Alvarez-Buylla, A. (1997). Direct evidence for homotypic, glia-independent neuronal migration. Neuron 18, 779-791.

Wiegreffe, C., Simon, R., Peschkes, K., Kling, C., Strehle, M., Cheng, J., Srivatsa, S., Liu, P., Jenkins, N.A., Copeland, N.G., et al. (2015). Bcl11a (Ctip1) Controls Migration of Cortical Projection Neurons through Regulation of Sema3c. Neuron 87, 311-325.

Yamawaki, N., Borges, K., Suter, B.A., Harris, K.D., and Shepherd, G.M. (2014). A genuine layer 4 in motor cortex with prototypical synaptic circuit connectivity. eLife 3, e05422.

Yamazaki, H., Sekiguchi, M., Takamatsu, M., Tanabe, Y., and Nakanishi, S. (2004). Distinct ontogenic and regional expressions of newly identified Cajal-Retzius cell-specific genes during neocorticogenesis. Proc Natl Acad Sci U S A 101, 14509-14514.

Yokoi, N., Namae, M., Wang, H.Y., Kojima, K., Fuse, M., Yasuda, K., Serikawa, T., Seino, S., and Komeda, K. (2003). Rat neurological disease creeping is caused by a mutation in the reelin gene. Brain research Molecular brain research 112, 1-7.

Yoshida, M., Assimacopoulos, S., Jones, K.R., and Grove, E.A. (2006). Massive loss of Cajal-Retzius cells does not disrupt neocortical layer order. Development 133, 537-545.

Yu, Y.C., Bultje, R.S., Wang, X., and Shi, S.H. (2009). Specific synapses develop preferentially among sister excitatory neurons in the neocortex. Nature 458, 501-504.

Zhao, S., Chai, X., Forster, E., and Frotscher, M. (2004). Reelin is a positional signal for the lamination of dentate granule cells. Development 131, 5117-5125. 


\section{Acknowledgements}

The PhD is not a solitary journey and many have contributed for this to happen. The past years in this group have been exciting on a professional level, but also on a personal note.

First of all, I would like to express my most sincere gratitude to my two mentors, Prof. Dr. Staiger and Dr. Robin Wagener. Their continuous support, their passion and dedication to science and their wealth of knowledge have been decisive for the development of the project and truly inspired me. It has been really pleasure to be trained by these two brilliant scientists and for this I cannot thank them enough.

I also acknowledge Prof. Dr. Thomas Dresbach and Prof.Dr. Ahmed Mansouri, members of my thesis committee, for the input and encouragement they provided me with.

The exceptional team of this department is definitely one of the highlights of these years. I am endlessly grateful for the excellent technical support provided by Patricia Sprysch, Sandra Heinzl and Anna Dudek. The efforts of Pavel Truschow were the sole reason why some of the experiments could be analyzed and for that deserves my gratitude. Thanks are also due to Dr. Julien Guy who took care of the mouse lines used in this study for the last years. I am also thankful to Dr. Tran Tuoc, Dr. Joachim Rosenbusch, Linh Phan and the rest of the members of Tuoc's group for adopting me in their seminars and their support when in need. I could not have been more fortune with my lab- and office-buddies, Ramanathan Narayanan and Kamila Kiszka. The frustration, happiness and laughter we have shared over these years will not be forgotten. I am especially thankful to Kamila. She is not only an outstanding PhD student, but also a constant source of support. Many thanks also to the rest of the members of department, who also contributed, in one way or another, to the culmination of this project.

I would not have come this far without the support of my family and friends. I have nothing else but an everlasting gratitude to my girls: for spoiling me every time I was around, for all their updates and for making me fell part of all those big days I have missed as an expat. Thanks are also due to my family in-law, the Gottingen squad, Maria and my favorite roomie, Eli. 
To my beloved half lemon, the person who infected me with the biologist-bug, and our favorite crew member: this PhD is especially dedicated to you.

All I am or I hope to be, I owe to my parents. Their wisdom, support and unconditional love have been decisive for this adventure, and for many others, to be successfully completed. I am immensely privileged to have you.

As to the best partner one could dream of, and because live is infinitely better since I share it with you, thanks Alberto for all these years and the ones to come.

Valentina: anything can happen, anything can be.

THANK YOU.

Nieves Mingo Moreno 


\section{Curriculum Vitae}

NIEVES MINGO MORENO

Address: Zimmermannstrasse, 13 - 37075 Goettingen, Germany

Email: nieves.mingo@med.uni-goettingen.de

Tel.: +49-551/39-33900

Born on the 01.05.1986 in Madrid, Spain

\section{Education}

2014 - present Doctorate in Neuroscience

Institute of Neuroanatomy, Georg-August University Goettingen (Germany)

GGNB - Molecular Physiology of the Brain (CMPB) PhD program

2010 - $2011 \quad$ MSc in Pharmacological Research

Specialization: Neuropharmacology

Autonomous University of Madrid, Madrid (Spain)

$2004-2010$ BSc (2:1 Honours) in Biology

Specialization: Genetics, Cell and Molecular Biology

5 year program (Licenciatura)

Autonomous University of Madrid, Madrid (Spain)

2009-2010 Bachillerato (Science)

Colegio Nuestra Señora de las Nieves, Madrid (Spain) 


\title{
Professional experience
}

\author{
2013 - present Research Assistant \\ Institute of Neuroanatomy \\ Georg-August University Goettingen (Germany) \\ 2011 \\ Student Research Assistant \\ Cell Biology and Development Department \\ Carlos III Health Institute (Spain) \\ 2010 \\ Student Research Assistant \\ Institute for Regeneration \\ University of Edinburgh, Edinburgh (United Kingdom) \\ 2009-2010 Student Research Assistant \\ Cell Biology and Development Department \\ Carlos III Health Institute (Spain)
}

\section{Posters}

N. Mingo-Moreno, R.J. Wagener and J.F. Staiger (2017). "From inside-out to outside-in: the reeler cortex shows distinct rostro-caudal gradients in preplate splitting and lamination". Cortical Development Conference (Chania, Greece)

N. Mingo-Moreno, J.F. Staiger and R.J. Wagener (2016). "Hiking across the brain: unique spatiotemporal patterns of neuronal migration and cortical layering in the reelin-deficient neocortex". $10^{\mathrm{TH}}$ FENS Forum of Neuroscience (Copenhagen, Denmark)

N. Mingo-Moreno, J.F. Staiger and R.J. Wagener (2015). "Neuronal migration and layer formation in the reelin- deficient neocortex". PhD CMPB student Symposium (Göttingen, Germany)

N. Mingo-Moreno, R. Wallrafen, J.F. Staiger and R.J. Wagener (2015). "Multiple roles of reelin in neuronal migration and layer formation: beyond the simplistic view". Scientific poster. $11^{\text {th }}$ German Neuroscience Society Meeting (Göttingen, Germany) and CDB Symposium (Kobe, Japan)

R.J. Wagener, N. Mingo-Moreno and J.F.Staiger (2014). "Thalamo-cortical target selection flexibility adapts to different patterns of cellular disorganization caused by the absence of reelin". Cortical Development Conference 2014 (Chania, Greece)

González-Gómez P, Díaz-Moreno M, Mingo-Moreno N, Hortigüela R, San Emeterio J and Mira H (2010). "MicroRNAs regulation of neural stem cell differentiation". EuroSyStem Consortium Meeting 2010 (Amsterdam, Holland) 


\section{Conferences and Symposiums}

PhD CNMPB student Symposium: Encephalon (Max Planck Institute Göttingen, Germany - 2017)

Cortical Development Conference (Crete, Greece - 2017)

$10^{\text {TH }}$ FENS Forum of Neuroscience (Copenhagen, Denmark - 2016)

PhD CNMPB student Symposium: Encephalon (Max Planck Institute Göttingen, Germany - 2015)

CDB Symposium: Time in Development (Riken Institute Kobe, Japan - 2015)

$11^{\text {th }}$ Meeting German Neuroscience Society (Göttingen, Germany - 2015)

Barrels Conference (Göttingen, Germany - May, 2014)

\section{Publications}

Mingo-Moreno, N., Dudek, A., Truschow, P., Staiger, J.F.*, and Wagener, R.J.*. From Inside-out to Outside-in: New Insights into the Cortical Lamination of the Reelin-deficient Neocortex. In preparation.

Wagener, R.J., Witte, M., Guy, J., Mingo-Moreno, N., Kugler, S., and Staiger, J.F. (2016). Thalamocortical Connections Drive Intracortical Activation of Functional Columns in the Mislaminated Reeler Somatosensory Cortex. Cereb Cortex 26, 920-837. In press.

\section{Awards}

$1^{\text {st }}$ Poster Price - Encephalon Symposium, 2015

Travel Fellowship - Center for Developmental Biology, Riken Institute Japan, 2015

Faro Grant - Graduate Research Scholarship, 2013

Goya Mundus Grant - Undergraduate Research Scholarship, 2010

High School Scholarship - Top class student Madrid, 2002 - 2003

\section{Additional information}

\section{Qualifications}

In utero electroporation, RNA in situ hybridization and probe generation

Brain isolation, tissue processing and immunofluorescence

Confocal Imaging

Protein purification, cloning, Western Blot and similar molecular techniques 
Cell culture proliferation and differentiation essays

Solid working knowledge of Microsoft Office Applications, Adobe Photoshop CS6, Adobe Illustrator, Adobe InDesign, CorelDraw X6, Neurolucida 11.0 and Sigma Plot.

Notions of Image J and R.

\section{Additional courses/accreditations}

FELASA, Category B - Georg-August University Goettingen (Germany, 2015)

Crispr-Cas9: from gene to function - Centre for Genomic Regulation (Spain, 2016)

\section{Languages}

Spanish native speaker. Fluent in English.

Notions for German, French and Italian. 